\title{
VII. Souveränität und Ambition: Die Transformation der Beziehungen im Kontext der Emanzipation Irans, 1964-1967
}

\author{
VII. 1. «Unabhängige Nationale Politik» und die frühe Umsetzung iranischen \\ Vormachtstrebens in der Golfregion, 1964-1967
}

Armin Meyers Einschätzung der sich aus der Ablösung des amerikanischen Militärmonopols im Jahr 1966 ergebenden Folgen betonte die langfristigen Vorteile einer Distanzierung vom früheren Patron. Eine unabhängigere iranische Politik vergrößere mittelfristig die Chancen für interne Stabilität in Iran und habe zugleich im Falle einer Rückkehr der Instabilität in der Zukunft den entscheidenden Vorteil, dass die amerikanische Macht nicht automatisch mit dem Ancien Régime identifiziert werden würde. Damit habe Washington die Chance, auch mit einer möglichen Nachfolgeregierung einvernehmliche Beziehungen zu etablieren. Voraussetzung dafür war, dass auch die breite Masse der iranischen Bevölkerung den neuen unabhängigen Kurs als reelle Widerherstellung voller iranischer Souveränität interpretiere und die lange Zeit vorherrschenden Vorstellungen einer Steuerung der eigenen Regierung durch ausländische Mächte - zuvorderst die USA, aber auch Großbritannien - ablege. Zur Vervollständigung des von Meyer erhofften Effekts wäre insbesondere eine Reduktion der amerikanischen Präsenz und eine unzweideutige Distanzierung gerade von den staatlichen Repressionsorganen der Schah-Diktatur vonnöten gewesen. Beides sollte nicht gelingen, wie die Folgejahre demonstrierten.

Die Distanzierung vom ehemaligen Patron auf dem Feld der Außenpolitik setzte sich auch unter der Johnson-Administration fort. Insbesondere die konsequente Fortsetzung des Ende 1962 eingeleiteten Rapprochements mit der ehemals als hauptsächlichen Bedrohung wahrgenommenen Sowjetunion erweckte den Eindruck einer nunmehr unabhängigen Orientierung nach außen. Auf die erstmalige Gewährung direkter Wirtschaftshilfe durch die UdSSR im Juli und den Besuch Breschnews in Iran im November 1963 folgte die seit 1957 ausstehende Ratifikation des Handels- und Transitabkommens, die Unterzeichnung eines Abkommens über die zivile Luftfahrt sowie die Aufnahme von Gesprächen über gemeinsame Entwicklungsprojekte. Nachdem die sowjetisch-iranische Annäherung im Laufe des Jahres 1964 ins Stocken zu geraten schien, war gerade aus amerikanischer Sicht die Ankündigung während des Schah-Besuchs in der UdSSR im Juni 1965, man habe sich auf den Bau einer Stahlhütte in Iran geeinigt, eine Überraschung. Einer amerikanischen Einschätzung zufolge wurde die Nachricht, dass die Sowjets diese Stahlhütte bauen würden, ,, received with genuine 
enthusiasm by the Iranian public.“1 Bereits Breschnew hatte offenbar bereits während seines Iran-Aufenthalts Ende 1963 ein solches Angebot unterbreitet, doch setzten die Iraner im Anschluss Verhandlungen auch mit westlichen Staaten fort. ${ }^{2}$ Angesichts der Attraktivität der angebotenen sowjetischen Konditionen und der in der politischen Folklore Irans fest verankerten Vorstellung, kapitalistische Interessen des Westen hätten den Bau einer Stahlhütte seit den 1930er Jahren verhindert, war der Abschluss mit Moskau im Januar 1966 von großer symbolischer Bedeutung. ${ }^{3}$ Die Sowjets gewährten einen günstigen Kredit über \$286 Millionen, dessen Rückzahlung mit Lieferung von (zuvor meist abgefackeltem) Erdgas erfolgen würde. $\mathrm{Zu}$ diesem Zweck sollte eigens als Teil der Unternehmung eine Gaspipeline nach Norden gebaut werden. So populär das Projekt in Iran war, so ungesichert war die Finanzierung der geschätzten Gesamtkosten von bis zu einer Milliarde US-Dollar. ${ }^{4}$

In Bezug auf das Stahlhüttenprojekt wurden die USA in der iranischen Öffentlichkeit sehr kritisch beurteilt, da Washington bereits 1961 ein frühes Kooperationsprojekt zum Bau einer Hütte, u.a. mit Demag-Krupp, verhindert hatte. Zwar interpretierte man dies in den USA als pro-iranische Geste - „[...] we saved the GOI \{Government of Iran; R.P.\} from a colossal industrial and financial disaster" ${ }^{\text {, }}$, doch wurde die Idee eines einheimischen Stahlhüttenprojekts in der Folge nur noch mehr zu einem Prestigeprojekt im Rahmen der Ablösung des Klientelstatus und zum Ausdruck der Durchsetzung der eignen industriepolitischen Autonomie vis-à-vis dem amerikanischen Patron. ${ }^{5}$ Gegenüber Botschafter Meyer begründete Mohammad Rezā die Annahme der sowjetischen Offerte denn auch mit der fortdauernden Ablehnung eines umfassenden Industrialisierungsprogramms durch Washington - Präsident Eisenhower habe ihm gegenüber persönlich das Verlangen von Ländern nach „damn steel mills“ verspottet. ${ }^{6}$ Offenbar hatten das Entgegenkommen Moskaus in der Frage

\footnotetext{
${ }^{1}$ Report, , The Trend toward Closer Soviet-Iranian Relations', 9.8.66, ,IRG/NEA', Lot 68 D 117, Subject Files 1965-66, ASS/NEA, Box 2; die Zwillingsschwester des Schahs, Prinzessin Ašraf, hatte bereits im Juni 1964 Moskau besucht.

${ }^{2}$ Vgl. Tehran \#A-297, 26.11.63, ,Bilateral Talks - Bonn - Talks on Counter Strategy', Lot 71 D 462, Subject Files, 1961-1963, Office of the Special Assistant to the Under Secretary of State for Political Affairs, Box 2; in diesem Dokument werden auch geheime Sitzungen der Planorganisation auf Basis von CIA-Berichten geschildert. Seit 1964 verhandelten die Iraner mit zwei US-Konsortien und zwei europäischen Konsortien über die Umsetzung des Projekts. Vgl. State \#CA-12609, 26.5.65; Tehran \#A-86 to State, 9.8.65, FN 9-1 IRAN, CF 64-66.

${ }^{3}$ Vergeblich waren daher ohnehin halbherzige Versuche, das Stahlhüttenprojekt durch westliche Firmen bzw. Konsortien umsetzen zu lassen. Vgl. Note ,Steel Plant Project - Iran', 23.9.65, encl. to Foy Memo, 23.9.65; Mann Memo, 5.8.65; Bracken Memo, 3.8.65, ,INCO - [...]Oct) 1965'; AID hatte sich bereits früh gegen ein „präemptives“"Vorgehen ausgesprochen. Vgl. Macomber Memo, 15.6.65; Tiger Memo, 27.5.65; Braderman Memo, 20.5.65, ,INCO - Iran \& Steel \& Related Products 1965', Lot 69 D 484, NEA/IRAN: 1964-66, Box 1; bereits im April 1960 hatte es eine Grundsatzvereinbarung über den Bau einer Stahlhütte mit einem deutschbritischen Konsortium unter Beteiligung der Demag-Krupp gegeben. Das Abkommen scheiterte an innenpolitischer Kritik, Gerüchten über Bestechungen und am Widerstand der Weltbank. Vgl. Cohn Memo, 7.9.65, POL 15-1 IRAN, CF 64-66; vgl. Meyer Letter to Howejdā, 3.10.65, ,Iran/October 1965 (1)`, Box 13, Meyer Papers, GUL.

${ }^{4}$ Vgl. CIA Special Report, 22.4.66, ,Iran, Memos \& Misc, Vol. 2 [2 of 2]', NSF, Country File, Box 136, LBJL; vgl. Tehran \#9, 4.7.65, FRUS 64-68, XXII, S. 165; vgl. Technical, Industrial and Financial Agreement between the USSR and Iran, 13.1.66, Annex Steel Mill, in: DMYTRyshyn/Cox, Soviet Union and the Middle East, S. 377392.

${ }^{5}$ Vgl. Walsh Memo, 9.2.65, ,INCO[...] Products 1965', Lot 69 D 484, Box 1; Walsh Memo, 20.11.64, ,INCO [...]Iron \& Steel 1964', Lot 69 D 95, NEA/IRAN: 1964-66, Box 3.

${ }^{6}$ Tehran \#244, 31.8.65, FRUS 64-68, XXII, S. 169; vgl. auch Tehran \#776, 25.11.65, ebd., S. 189-93; Telcon [Ball, Crawford], 3.8.65, ,Iran [2/14/65-4/26/66] ${ }^{\circ}$, Papers of George Ball, Box 4, LBJL; vgl. auch Gratian
} 
der Stahlhütte, aber auch der ihm zuteil gewordene pompöse Empfang in der sowjetischen Hauptstadt, beim Schah Eindruck hinterlassen. ${ }^{7}$ Gegenüber Meyer erwähnte er bereits hier sowjetische Offerten hinsichtlich Militärhilfe wie z.B. MiG-Kampfflugzeugen, und auch den offenbar von sowjetischer Seite wieder ins Spiel gebrachte Abschluss eines Nichtangriffspaktes zwischen beiden Staaten für einen Zeitraum von 200 Jahren - ein ähnliches Angebot hatte Moskau bereits 1959 unterbreitet. $^{8}$ Wie üblich, waren die Schilderungen sowjetischer Großzügigkeit auch immer darauf gerichtet, ein möglichst weitreichendes amerikanisches Entgegenkommen in militärischen Frage zu generieren. ${ }^{9}$

Ohne Zweifel aber bestärkte der Erfolg seiner Moskau-Politik den Schah in seiner Überzeugung, seine unabhängige Politik weiter fortzusetzen. Der nächste, bereits geschilderte Schritt war im darauffolgenden Jahr die Aufnahme von Verhandlungen über ein Waffengeschäft mit der UdSSR. ${ }^{10}$ Moskaus Position gegenüber Iran hatte sich seit Chruschtschows Aussage gegenüber Walter Lippmann von 1961, das Land werde wie ein reifer Apfel in den sowjetischen Schoß fallen, in der Tat grundlegend gewandelt. Ende 1966 war man sich auch innerhalb NEAs im Klaren, dass „Khrushchev's ripe apple metaphor is evidently out of date." Offenkundig aber war man weiterhin darüber verblüfft, wie weitreichend die sowjetische Bereitschaft zur Kooperation mit dem "Imperial House of Pahlavi“" war. ${ }^{11}$ Gleichzeitig baute Iran seine Beziehungen zu den anderen Ländern des Ostblocks systematisch aus, insbesondere zu Rumänien. Die US-Botschaft vermutete, der Schah sähe in dem Land einen „Ex-Satelliten“ Moskaus und damit eine Positionierung im internationalen System äquivalent zu der Irans, was eine enge Kooperation besonderes geboten erschienen ließ. ${ }^{12}$

Während die Annäherung an Moskau im Grundsatz seitens der USA wenn nicht begrüßt, so doch toleriert wurde, waren andere Aspekte der neuen iranischen Außenpolitik weitaus schwieriger in die amerikanische Grand Strategy für die Nahostregion zu integrieren. Insbesondere die fortwährende Verschlechterung der Beziehungen Irans zu seinen arabischen

\footnotetext{
Yatsevitch OHI, 5.11.88, OHIC, S. I-132f.: ,[...] to be a country of any significance, you had to have a steel mill."; Moskau habe Iran zudem mit Technologie der 1920er abgespeist.

${ }^{7}$ Vgl. Tehran \#A-29, 8.7.65, POL 15-1 IRAN, CF 64-66.

${ }^{8}$ Vgl. Tehran \#319, 9.9.65, FRUS 64-68, XXII, S. 171; Eintrag vom 16.5.69, in: AlAM, Shah and I, S. 63f.; vgl. oben S. 199f.

${ }^{9}$ Mit der Quellenlage nicht vereinbar die These bei JoHns, Changing Patterns, S. 73.

${ }^{10}$ Noch im März 1966 hatte die CIA in einem NIE zu Iran angekündigt: „Despite his desire for additional military equipment, the Shah would be most reluctant to accept even token amounts of Soviet equipment."; CIA, NIE 3466, ,Iran', 24.3.66, ,34, Iran“, NSF, National Intelligence Estimates, Box 6, LBJL; das grundsätzliche Misstrauen gegenüber Moskau bestand aber fort. Die Fortsetzung der Attacken gegen den Schah durch die ,geheimen“" Radiosender tat ein Übriges. Im Frühjahr 1966 wies Iran auch drei sowjetische Diplomaten aus, die angeblich versucht hatten, den SAWAK zu penetrieren. Vgl. ,Thumbnail Guidance for Talk with Shah', encl. to Meyer Letter, 18.4.66, ,IRG/NEA', Lot 68 D 117, Subject Files 1965-66, ASS/NEA, Box 2; Tehran \#A-697, 8.4.66, POL IRAN-USSR, CF 64-66.

${ }^{11}$ Eliot Letter, 20.12.66, ,POL IRAN/USSR IRAN 1966‘, Lot 70 D 330, NEA/IRN: 1964-66, Box 17.

${ }^{12}$ Vgl. Tehran \#A-164, 22.8.66, POL 2-3 IRAN; im Spätsommer 1966 unternahm der Schah z.B. eine Serie von Visiten in Ungarn, Bulgarien und Polen. Vgl. Tehran \#A-207, 13.10.66, POL 7 IRAN, CF 64-66; ab ca. 1966 entwickelte sich auch der Handel mit der DDR, war jedoch aufgrund der Haltung Ost-Berlins bez. Iraks politisch bis in die frühen 1970er belastet. Siehe Vermerk für Gen. Sölle, 28.9.70; Vereinbarte Niederschrift [Sölle, Ansāri], März 1973, MfAA C 542/75, PAAA; vgl. ZíDEK, La Tchécoslovaquie communiste et l'Iran; STANCIU, Romania's Policy in the Middle East; Alvandi/GHEORGHe, Petro-Diplomacy with Ceausescu.
} 
Nachbarn war zunehmend Grund für Besorgnis. Bereits in seinem Brief an den neuen Präsidenten Johnson hatte der Schah vor dem Aufbau einer ägyptischen Interventionsstreitmacht gewarnt, die auch die Sicherheit Irans gefährden könne. ${ }^{13}$ Die Johnson-Administration war sich der begrenzten ägyptischen Möglichkeiten zur militärischen Machtprojektion bewusst und verstand die Besorgnis des Schahs in erster Linie als taktischen Richtungswechsel, um auch nach der Verbesserung der eigenen Beziehungen mit Moskau eine Begründung für fortgesetzte Aufrüstung zu haben. Zudem sah man die wiederholten, und teilweise hanebüchenen Behauptungen Mohammad Rezās über ägyptische Intentionen nicht zuletzt als Ausdruck des israelischen Einflusses auf den iranischen Verbündeten. ${ }^{14}$ Aus amerikanischer Sicht war eine Konfrontation zwischen Nassers VAR und Iran alles andere als willkommen, stand doch die eigene begrenzte Disengagement-Politik gegenüber Kairo allein schon durch die ägyptisch-saudische Konfrontation im Jemen-Konflikt unter erheblichem Druck. Für Washington bestand zudem die Gefahr, dass der Schah, ,in his preoccupation with Nasser [...] may minimize long-range Soviet designs on Iran. “15

Aus Sicht Washingtons befand sich Iran inzwischen in einer vorteilhaften strategischen Position, doch wollte Mohammad Rezā diese günstige Einschätzung naturgemäß nicht teilen. ${ }^{16}$ Der Schah betonte die pan-arabischen Ambitionen Nasser auf die iranische Provinz Xuzestān, zugleich das Zentrum der Ölförderung und damit ökonomische Basis der eigenen staatlichen Macht. Arabische Einheitsbestrebungen - trotz der gerade erst gescheiterten ägyptischirakisch-syrischen Dreier-Union -, umfangreiche sowjetische Waffenlieferungen, ägyptische Aktivitäten in Südarabien, fortwährende Propagandaattacken gegen die Person des Schahs und auch Iran im Allgemeinen, die Verwendung der Bezeichnungen „Arabischer Golf“ und auch „Arabistan“ anstelle von Xuzestān, der vorgeblich unmittelbar bevorstehende Rückzug der britischen Macht - all dies führte der Schah als Beleg für die Ernsthaftigkeit der Bedrohung an. Er gestand zudem nicht ein, dass die eigene Politik Irans wie z.B. die CENTO-Mitgliedschaft, seine pro-westliche Ölpolitik, die engen Beziehungen mit Israel oder die ungezügelte Einwanderung von Iranern in die arabischen Scheichtümer entlang der Westküste des Golfs seitens der Araber als Bedrohung angesehen werden könnten. Vergeblich versuchte Washington Iran von seiner konfrontativen Haltung abzubringen:

We have agreed with the Shah that Iran has valid reason to be concerned over the next decade and beyond with the potential impact of Arab nationalism on his border territories. But we try

\footnotetext{
${ }^{13}$ Vgl. oben S. 427, Anm. 120.

${ }^{14}$ Vgl. Macy Memo, 22.1.64, ,POL 15-1 IRAN Head of State Iran 1964‘, Lot 69 D 178, NEA/IRN: 1964-66, Box 4; Außenminister Ārām beschwerte sich gegenüber den Amerikanern über den ansteigenden Einfluss einer proIsrael-Lobby in Iran. Vgl. Tehran \#117, 30.7.64, POL 13 IRAN, CF 64-66.

${ }_{15}$ Report, ,[...]Soviet-Iranian Relations', 9.8.66, ,IRG/NEA', Lot 68 D 117, ASS/NEA: Subject Files 1965-66, Box 2; Mitglieder des ehemaligen pro-amerikanischen Netzwerks wie z.B. Xodādād Farmānfarmā'ijān, inzwischen in der Zentralbank beschäftigt, zeigten sich besorgt über die Verhandlungen mit den Sowjets. Vgl. MemCon, 22.9.65, ,POL 15-1 Head [...] 1965', Lot 70 D 552, NEA/IRN: 1965-75, Box 1; Tehran \#A-378, 16.1.65, ,Iran - Airgram/Reports, 1963-1967', Part 2: Box 1a, Herz Papers, GUL.

${ }^{16}$ Vgl. Background Paper, ,Concern with 'Arab Threat', 26.5.64, ,POL 15-1A', Lot 69 D 178, NEA/IRN: 196466 , Box 4.
} 
to persuade him of our conviction that the potential threat is political and psychological rather than military and can best be countered by internal measures designed to improve the circumstances of Iran's border minorities. We have cited Arab military weakness and lack of unity, particularly in view of the common threat posed by Israel, and our own warnings against aggression in the area. We have indicated more faith in British staying power than the Iranians have. Finally we have counselled the Iranians to capitalize upon suspicions of Nasser's aims among Iran's Arab neighbors, and to try to avoid provocative behavior toward them. ${ }^{17}$

Mohammad Rezā befolgte den amerikanischen Rat nicht. Für ihn war Nasser ,the root of all Mideast evil.“18 Versuche, den Schah zumindest hinsichtlich der sehr begrenzten militärischen Bedrohung durch die Araber $\mathrm{zu}$ überzeugen, fruchteten ebenfalls nicht. ${ }^{19}$ Die Propagandaschlacht zwischen Kairo und Teheran im Laufe von 1964 nahm somit an Heftigkeit zu und weitete sich bald auf die Golfregion aus. Premierminister Mansur begründete vor dem Mağles den ersten Militärkredit über \$200 Millionen zuvorderst mit der ägyptischen Bedrohung, woraufhin Nasser den Schah öffentlich als „Handlanger des Zionismus“ bezeichnete. ${ }^{20}$ Die Entsendung eines symbolischen VAR-Truppenkontingents in den Irak im September löste beim Schah zusätzliche Besorgnis aus - er bestand auf gemeinsamen Ernstfallplanungen mit den USA und Großbritannien für den Fall einer nasseristischen Machtübernahme in Bagdad. ${ }^{21}$ Der Schah verglich die amerikanische Unterstützung SaudiArabiens gegen die VAR unvorteilhaft mit der gegenüber Iran. Laut einem CIA-Bericht beschwerte sich der Schah in einem Wutanfall intern: „If only the United States would support us as well as its supports Faysal.“22 Washington beschwichtigte und vermied es zugleich, in die iranischen Aktionen gegen Irak impliziert zu werden. ${ }^{23}$ Dabei zeigte sich auch hier wieder die zunehmende Erosion des latenten anglo-amerikanischen Kondominiums. Komer

\footnotetext{
${ }^{17}$ Ebd.

${ }^{18}$ Tehran \#A-593, 24.2.66, POL 15-1 IRAN, CF 64-66; in einem Gespräch mit Harriman nannte Mohammad Rezā Nasser gar ein „bloodthirsty monster“. Siehe Further Notes on Conversation with the Shah, 5.6.67, ,Shah of Iran“, Box 488, Harriman Papers, LOC.

${ }^{19}$ Vgl. DIA Estimate, o.D., encl. to Thomas Letter, 27.3.64, ,POL - Iran/Arab States (General) IRAN 1964', Lot 69 D 178, NEA/IRN: 1964-66, Box 5; im Juni 1965 genehmigte die Administration die Weitergabe von Geheimdienstinformationen hinsichtlich der tatsächlichen Stärke der ägyptischen, syrischen und irakischen Luftwaffen an Iran, trotz Bedenken hinsichtlich der geheimdienstlichen Zusammenarbeit zwischen Iran und Israel. Vgl. Lee Memo, 23.6.65, kein Folder, Lot 69 D 489, ebd., Box 11; man vermutete zudem, dass der israelische Geheimdienst Beweise gefälscht habe, um einen endgültigen Bruch zwischen dem Schah und den Arabern zu provozieren. Vgl. Grindall Memo, 13.1.64, ,POL 13-3-a Arab Tribes (Khuzistan) IRAN 1964', Lot 69 D 225 , ebd., Box 6; siehe den Eintrag vom 11.6.64, in: LILIENTHAL, Journals: VI, S. $42 \mathrm{ff}$.

${ }^{20}$ Vgl. Tehran \#236, 10.11.64, DEF 1 IRAN; Tehran \#A-264, 23.11.64, POL IRAN-UAR; Cairo \#A-451, 30.12.64, POL IRAN-UAR, CF 64-66.

${ }^{21}$ Vgl. MufTI, Sovereign Creations, S. 170; das State Department willigte widerwillig in begrenzte Beteiligung von ARMISH/MAAG in nationale iranische Ernstfallplanungen für den Fall eines irakischen Angriffs und in nachrichtendienstlichen Austausch mit den Iranern ein. Vgl. State \#443, 21.11.64, DEF 1-1 IRAQ; Tehran \#570, 19.11.64, DEF 1-1 IRAN, CF 64-66; vgl. JCS \#9409, 3.10.64, ,Iran, Volume 1, 1/64-12/65‘, NSF, Country File, Box 136, LBJL; im Gegensatz dazu waren die Briten bereit, den Forderungen des Schahs weitgehend entgegenzukommen, offenbar infolge ihrer eigenen, immer aggressiver werdenden antinationalistischen Politik gegenüber der VAR. Die USA verweigerten die vorgeschlagene Durchführung von „limited war planning“ im Rahmen der CENTO und waren auch sonst vorsichtig hinsichtlich der iranischen Absichten. Vgl. MemCons, 24./30.9.64, DEF 1-1 IRAN, CF 64-66; Washington Embassy to FO, No. 3099, 2.9.64; FO to Washington Embassy, No. 11558, 25.9.64, PREM 11/4926; JIC(A)(69)48(Final), 10.12.69, CAB 186/3, TNA:PRO.

${ }_{22}$ CIA Information Cable \#14458, 11.5.66, ,Iran Cables Vol. 2 [2 of 2] ${ }^{6}$, NSF, Country File, Box 136, LBJL; die Quelle für die CIA war der ehemalige Ministerpräsident Šarif-Emāmi.

${ }_{23}^{23}$ Jede Beratungstätigkeit von ARMISH/MAAG sollte gemeinsame Bedrohungseinschätzungen und abgesprochene Gefahrenlagen vermeiden, ein starker Kontrast zum späteren Peterson-Report. Vgl. Mulligan Memo, 25.3.66, ,POL IRAN/IRAQ (March- ) IRAN 1966“, Lot 70 D 330, NEA/IRAN: 1964-66, Box 17.
} 
beschwerte sich explizit über die britische Haltung, die den Schah in seinen überzogenen Sicherheitsbedenken wegen der - im Kern nur symbolischen - militärischen Präsenz der VAR im Irak noch bestärkte. ${ }^{24}$

Die kurzzeitige Landung einer kleinen Aufklärungsmission auf der zwischen Iran und dem Emirat Sharjah (Al-Šāriqa), selbst Teil der britisch dominierten „Vertragsküste“, umstrittenen Insel Abu Musā am westlichen Eingang zur Straße von Hormus und die Etablierung einer ständigen Garrison auf der weiter westlich gelegenen, ebenfalls umstrittenen Insel Sirri, im Sommer 1964 führte zu einem Aufschrei arabischer Nationalisten, propagandistisch gefördert seitens der VAR. Das wachsende militärische Interesse Irans am südlichen Golf war zudem ein Zeichen, dass „the Iranian authorities have lately begun to behave somewhat more aggressively toward the Arabs in the Gulf than in the recent past. “25 Abu Musā sollte mit dem britischen Abzug aus dem Golf 1971 zu einem zentralen Streitpunkt zwischen Iran und den arabischen Staaten werden. Durchweg war man sich auf amerikanischer Seite zugleich der hegemonialen Ambitionen der Iraner in der Golfregion bewusst - nicht wenige sahen die ständigen Hinweise auf die ,,arabische Gefahr“ im Golf als Vorwand für die Ausdehnung des eigenen Einflusses. „He feels that this is an area where, as a long term policy, Iran can establish a position of prestige and power in the Middle East", so hatte bereits Holmes im September 1963 die Ambitionen des Schahs im Persischen Golf charakterisiert. ${ }^{26}$ Andererseits war man aber auch bereit, die iranischen Sicherheitsbedenken ernst zu nehmen. Historisch waren die kleineren politischen Entitäten auf der gegenüberliegenden Seite der Golfküste immer unter der Herrschaft stärkerer Mächte gestanden und nun, angesichts des sich abzeichnenden oder bereits bestehenden Ölreichtums, in der Tat ein ,great prize“. Die eigenmächtigen Aktionen der Iraner im Golf seien nicht zuletzt auf die Weigerung Großbritanniens, aber auch der USA, zurückzuführen, die zukünftige Sicherheitsordnung im Persischen Golf mit der Regierung in Teheran überhaupt zu diskutieren. ${ }^{27}$

Destabilisierend auf das iranische Verhältnis zu den arabischen Nachbarn wirkte zudem die Vertiefung der Beziehungen zu Israel in dieser Zeit. Amerikanische Offizielle vermuteten

\footnotetext{
${ }^{24}$ Vgl. Komer Memo, 2.9.64, ,Near East, Volume 1, 3/64-8/67‘, NSF, Country File, Box 116, LBJL.

${ }^{25}$ Khorramshahr \#A-1, 7.7.64, DEF 15 IRAN; Tehran \#863, 30.3.64, POL 32-6 IRAN-KUW; Baghdad \#A-48, 21.7.64, POL 1 IRAN-IRAQ; die Iraner formten eine eigene Persian Gulf Division im Außenministerium und verstärkten ihre Interaktionen mit den verschiedenen Scheichtümern, aber auch mit Kuwait. Größte Schwierigkeit war das Aufrechterhalten des eigenen Anspruchs auf Bahrain, der auch in offiziellen Karten wiedergegeben wurde. Zudem waren weiterhin zwei Sitze im Mağles für Bahrain reserviert, was immer wieder für Spannungen mit den Golfarabern führte. Unklar war, ob die neue Division ein Ende der Zuständigkeit der SAWAK bedeutete (,SAVAK $[\ldots]$ had been very active in the Persian Gulf area and had worked closely with Israeli intelligence."). Tehran \#A-461, 27.2.64, POL 1 IRAN, CF 64-66; zu den Golfinteressen Irans vgl. RAMAZANI, Persian Gulf, S. 33-41, 69-87; zur Rolle des SAWAK in der iranischen Außenpolitik in dieser Phase am Beispiel Libanons vgl. SAMII, Lebanon Policy, S. 72-77.

${ }^{26}$ Holmes Letter, 4.9.63, , Iran, 1/63-11/63 [Folder 1 of 2] ', NSF, RWK, Box 424, JFKL.

${ }^{27}$ Memo ,Potential Policy Problems in Iran Through 1968', 6.10.64, , Office Memoranda', Lot 69 D 30, NEA/IRN: 1964-66, Box 1; die Regierung bemühte sich in der Folgezeit um eine stärkere Integration der abgelegenen Häfen und Golfinseln in die iranische Nation und ein Zurückdrängen der arabischen Sprache entlang der Golfküste. Vgl. Khorramshahr \#A-63, 9.3.65, POL 19 IRAN, CF 64-66; vgl. Defence Review Working Party, 28.9.67, BDEE A, 5, I, S. 403-19; vgl. auch ZABIH, Iran's Policy.
} 
fortwährend, dass nicht wenige der Fehlperzeptionen Mohammad Rezās bezüglich der „arabischen Gefahr“ auf israelisches Soufflieren zurückzuführen sei. Zugleich registrierte man in Washington ein zunehmendes Interesse Israels am Persischen Golf und der arabischen Halbinsel. Israelische Diplomaten priesen den Schah als Anker pro-westlicher Stabilität im Nahen Osten und drängten die USA auf bedingungslose Unterstützung, gerade was Entgegenkommen in den Rüstungsfragen anging: ,[...] the GOI \{Government of Israel; R.P.\} would view continuation of a fruitful relationship between the U.S. and Iran as a most important thing. “28 $\mathrm{Zu}$ diesem Zeitpunkt machte sich auch der zunehmende Einsatz der immer einflussreicher werdenden Pro-Israel-Lobby zugunsten iranischer Interessen in Washington bemerkbar. Pro-israelische Institutionen in den USA unterstützten diverse iranische Kampagnen, gerade bezüglich der Aufrüstungswünsche Mohammad Rezās, explizit und sorgten unter anderem für eine wohlwollende Berichterstattung während des USA-Besuchs des iranischen Monarchen im Juni 1964. ${ }^{29}$ Trotz der israelischen Unterstützung für iranische Wünsche verweigerte der Schah die de facto-Anerkennung Israels, in erster Linie um seinen Handlungsspielraum gegenüber den arabischen Staaten nicht vollständig aufgeben zu müssen. ${ }^{30}$

Ende 1965 erfuhren die USA zudem von Plänen Israels und Irans, gemeinsam eine Pipeline mit gewaltiger Kapazität vom israelischen Hafen in Eilat am Roten Meer bis zum Mittelmeer zu bauen. Basierend auf damals nicht verfolgten israelischen Plänen aus der Mitte der 1950er, wiederaufgelegt nach der Öffnung der Straße von Tiran im Anschluss an den Zweiten ArabischIsraelischen Krieg von 1956, hatten beide Staaten 1958 eine heimliche Übereinkunft über Öllieferungen nach Eilat getroffen. Von nun an deckte Iran ein Gros des israelischen Erdölbedarfs. Im Anschluss an die Blockade des Suezkanals nach 1967 wurde auch die Idee einer ,großen“ Pipeline vom Roten Meer zum Mittelmeer wieder aktuell. Nach schwierigen Geheimverhandlungen einigten sich Iran und Israel im Februar 1968 schließlich auf eine Erweiterung der bestehenden Verbindung mit einer Pipeline mit 42 Zoll Durchmesser, Gesamtinvestitionen von etwa \$120 Millionen umfassend. Für 1975 schätzte die CIA die

\footnotetext{
${ }^{28}$ MemCon, 12.7.66, ,POL IRAN/U.S. Iran 1966', Lot 70 D 330, NEA/IRN: 1964-66, Box 17; Gazit, der Assistant Director-General im israelischen Außenministerium, bezeichnete Iran als das dritte regionale Kraftzentrum neben Israel und der VAR. Er erwähnte auch die Tatsache, dass Israel 5 Millionen Tonnen iranisches Öl pro Jahr erhalte, von denen es nur 2 Millionen selbst konsumiere, inzwischen eine bedeutende Alternative zur Route durch den Suezkanal. Dies sei ein ,vital interest“ Israels. Tel Aviv \#A-106, 1.8.66; State \#CA-2548, 30.9.66, POL IRANISR, CF 64-66; zu den israelisch-iranischen Beziehungen Mitte der 1960er Jahre vgl. PARSI, Treacherous Alliance, S. 29ff.; SoBHANI, Pragmatic Entente; zur israelischen Strategie der Allianz mit der Peripherie im Allgemeinen vgl. AlPHER, Periphery, S. 13-6.

${ }_{29}$ Vgl. Bracken Memo, 5.10.65, POL IRAN-ISR, CF 64-66; in den 1970ern organisierte der Schah mit Unterstützung der Pro-Israel-Lobby PR-Kampagnen zur Beeinflussung der amerikanischen Berichterstattung über Iran. Siehe die Einträge vom 1.10.75, 20.1.76, 22.11.76, in: ALAM, Shah and I, S. 445f.; 463f.; 523; die JohnsonAdministration unterstützte die Geheimhaltung der bilateralen Beziehungen zwischen Israel und Iran. So erlaubte man z.B. die Nutzung der eigenen Kommunikationskanäle, um einen Bericht der Washington Post über ein Geheimtreffen des Schahs mit Levi Eshkol zu verhindern. Vgl. Tehran \#1754 [Al Friendly to Washington Post], 28.6.66, , Iran Cables, Vol. 2 [2 of 2]', NSF, Country File, Box 136, LBJL.

${ }^{30}$ Vgl. MemCon, 2.4.65, Asnād 11, S. 48; Botschaft Teheran an AA, 28.7.60, B 12/1007, PAAA; RAMAZANI, Iran and the Arab-Israeli Conflict; eng blieb allerdings die geheimdienstliche Kooperation mit Israel und der Türkei im Rahmen der TRIDENT-Allianz. So beschrieb ein CIA-Offizieller die Anwesenheit hochrangiger Geheimdienstler dieser Staaten in Teheran während seines Besuchs. Vgl. Critchfield Memo, 16.11.67, FRUS 6468, XXII, S. 439ff.; weitere Freigaben zuvor klassifizierter Teile dieses Dokuments infolge eines FOIA-Antrags (F-2013-00185) des Verfassers.
} 
Kapazität auf 50-60 Millionen Tonnen jährlich, wobei die geheimdienstlichen Analysten wie auch die meisten internationalen Ölexperten große Zweifel an der Wirtschaftlichkeit des Projekts hegten, zumal außer der NIOC keine andere staatliche oder private Ölgesellschaft bereit war, das politische Risiko einer Nutzung der Verbindung einzugehen. Sowohl die Sicherung des Energiebedarfs Israels wie auch der augenfällige Versuch mittels der EilatAschkelon-Pipeline wirtschaftlichen und politischen Einfluss der arabischen Produzenten zu unterlaufen, trugen zu einer weiteren Verschlechterung der Beziehungen Teherans zu den arabischen Staaten bei. ${ }^{31}$

Die Konstruktion einer „arabischen Gefahr“ richtete sich in der öffentlichen Propaganda Irans auf Nasser und die VAR, die konkreten iranischen Maßnahmen aber betrafen vorwiegend den irakischen Nachbarn, der als wahrscheinlichstes Einfallstor des Nasserismus in die Golfregion porträtiert wurde. ${ }^{32}$ Auch nach dem Sturz der Baath und der Machtübernahme von 'Abd asSalām 'Ārif verbesserten sich die Beziehungen zwischen Teheran und Bagdad nicht, nicht zuletzt aufgrund der weiterhin bestehenden Instabilität im Irak. ${ }^{33}$ Hauptstreitpunkte waren territorialer Natur, die ungelöste Šațț al-'Arab-Frage, Uneinigkeit über die exakte Abgrenzung der jeweiligen Hoheitsgewässer und Anschlusszonen und die damit zusammenhängende Vergabe von Offshore-Konzessionen, die seitens der Iraker immer wieder propagandistisch genutzte „Arabistan“-Frage hinsichtlich historischer Ansprüche auf Xuzestān, Gerüchte über den Anschluss Irakisch-Kurdistans an Iran und die gemeinsame Nutzung des grenzüberschreitenden Hānaqīn-Ölfeldes. ${ }^{34}$ Auf die fortgesetzte, wenn auch noch zurückhaltende, iranische Einmischung in den Kurdengebieten reagierte Irak mit der Diskriminierung der etwa 150.000 in Irak lebenden Iraner und der fortgesetzten starken Beschränkung von Pilgerreisen zu den schiitischen heiligen Stätten. Selbst die beiden USBotschaften vor Ort konnten sich nicht darauf einigen, welche der beiden Regierungen die Hauptverantwortung für den sich eskalierenden Konflikt trage. ${ }^{35}$ Die Botschaft in Teheran sah keine Möglichkeit für die iranische Regierung, ein Rapprochement mit Bagdad einzuleiten,

${ }^{31}$ Vgl. Tehran \#689, 6.11.65, PET 6 IRAN, CF 64-66; CIA Intel Memo ER IM 68-40, April 1968, DDRS: CK3100244093; vgl. BIALER, Eilat-Ashkelon Oil Pipeline; FARMANFARMAIAN, Blood and Oil, S. 356f., behauptet, die Vereinbarung sei auf amerikanischen Druck hin erfolgt. Die Quellenlage spricht gegen diese Behauptung.

${ }^{32}$ Dies bedeutet allerdings nicht, dass es ohne Involvierung der VAR keinen Konflikt zwischen Irak und Iran gegeben hätte. Die Vielzahl der territorialen und politischen Konflikte zwischen beiden Staaten machten einen Zusammenstoß sehr wahrscheinlich: „Iraqi-Iranian friction is a self-generating product of conflicting ambitions, nursed today by the Israelis, and requires no stimulus from the UAR."; ,Comments on UK Paper of August 20", o.D. [September 1964], POL 1 NEAR E, CF 64-66.

${ }^{33}$ Die CIA berichtete bereits im Januar 1964 von neuen Coup-Planungen der nationalen wie auch der regionalen Baath-Führungen gegen Präsident ' $\bar{A}$ rif. Vgl. Helms Memo for DCI, 3.1.64, , Iraq, Cables \& Memos, Vol. I, 12/63$7 / 65^{\circ}$; den Informationen der CIA zufolge hatte die Baath einige Gefolgsleute in der Präsidentengarde platziert und plane die Ermordung 'Ārifs, z.B. durch Sabotage seines Flugzeuges - 'Ārif starb 1966 bei einem Flugzeugabsturz. Wie zuvor mit der Baath-Regierung wurde jedoch die gegenseitige Auslieferung von Kommunisten beider Länder weiterhin fortgesetzt. Vgl. Khorramshahr \#A-38, 29.2.64, POL 23-6 IRAN-IRAQ, CF 64-66; vgl. auch die interessanten Ausführungen zu freigegebenen SAWAK-Dokumenten zu Irak in FARZANEH, Interregional Rivalry, S. 397ff.

${ }^{34}$ Die Iraner reagierten später auf die provokante „Arabistan“-Bezeichnung für Xuzestān mit der Umbenennung des Šațt al-'Arab in Arwand. Vgl. Jones Letter, 2.6.65, ,POL 33-1-b [...]1965', Lot 69 D 489, NEA/IRN: 196466, Box 11; zur aktiveren Kurdenpolitik Irans vgl. Davies Memo, 2.7.64, , Inter-Office Memos‘, Lot 66 D 5 , NEA/NR: Subject Files, 1958-1966, Box 4.

${ }^{35}$ Vgl. Bagdad \#A-1003, 11.6.64; Tehran \#A-734, 30.6.64, POL 1 IRAN-IRAQ, CF 64-66. 
solange sich Irak nicht von der „Vormundschaft“ der VAR gelöst habe, damit explizit die Argumentation des Schahs übernehmend, während der Botschafter in Bagdad argumentierte, fortgesetzter Druck der Iraner - und insbesondere das Aufwiegeln der kurdischen Minderheit trotz vereinbarten Waffenstillstands mit der Zentralregierung - mache eine Loslösung 'Ārifs von Kairo geradezu unmöglich. ${ }^{36}$

Gegenüber Secretary Dean Rusk verteidigte der Schah im April 1965 entschlossen seine regionale Politik und betonte ein weiteres Mal die seiner Meinung zufolge zu Besorgnis Anlass gebende Sicherheitslage im Persischen Golf. Wieder verwies er auf die Gefahr einer unmittelbar bevorstehenden Machtübernahme Nassers im Irak. Angesichts dieser Bedrohung sei er entschlossen, die Kurden als „Trumpfkarte“ in der Hinterhand zu halten. ${ }^{37}$ Inzwischen mehrten sich die Indizien, dass die seit 1963 vorgeblich zurückhaltende iranische Politik bezüglich der irakischen Kurdenfrage mehr als nur „Duldung“ kurdischen Widerstands beinhaltete. Im Anschluss an die Spaltung innerhalb der Demokratischen Partei Kurdistans im Sommer 1964 zwischen dem dominanten Bārzāni-Flügel und dem Ṭalabāni-Ahmed-Flügel wurde iranische Einmischung offenkundig, als die Anhänger des unterlegenen letzteren Flügels mit Unterstützung iranischer Stellen ins Nachbarland flohen. Der amerikanische Konsul in Täbris spekulierte gar, ,[...] that for some time past the Shah has been nursing in the back of his mind a secret hope of ultimately incorporating Iraqi Kurdistan into Iran. "38 Trotz irakischer Bemühungen, eine Detente im Verhältnis zu Teheran herbeizuführen, verschlechterten sich die Beziehungen weiter. ${ }^{39}$ Vergeblich bat die Regierung in Bagdad die USA um Hilfe angesichts der wiederholten Verletzungen irakischer Souveränität durch die Iraner - zugleich warnten sie vor möglichen militärischen Gegenreaktionen, sollte die Unterstützung der Kurden nicht unterbunden werden. ${ }^{40}$ Im Dezember 1965 kam es $\mathrm{zu}$ ersten irakischen Angriffen auf Stellungen der iranischen Gendarmerie entlang der Grenze zwischen beiden Ländern,

\footnotetext{
${ }^{36}$ Vgl. Tehran \#A-734, vorherig. Anm.; vgl. Tehran \#5, 2.7.64; Baghdad \#20 to State, 6.7.64, POL 1 IRAN-IRAQ, CF 64-66.

${ }^{37}$ Vgl. Tehran \#Secto30, 8.4.65, FRUS 64-68, XXII, S. 136f.; der Schah erklärte zudem seine Entschlossenheit trotz arabischer Proteste an seiner pro-israelischen Politik festhalten zu wollen. Der „Feind meines Feindes ist mein Freund“", so der Schah explizit.

${ }^{38}$ Coon Jr. Letter, 16.8.64, ,POL 13-3-c Kurdish tribes IRAN 1964', Lot 69 D 225, NEA/IRN: 1964-66, Box 6; vgl. REISINEZHAD, Iraqi Kurds and Lebanese Shia, S. 108-33; LitTLE, United States and Kurds, S. 71 ff., behauptet eine Entscheidung Ende 1964 der CIA zugunsten der Unterstützung iranischer und israelischer subversiver Operationen in den Kurdengebieten. Die zur Verfügung stehende Quellenlage erlaubt keine Aussage über amerikanische Ermunterung - zumindest hätte diese der ansonsten verfolgten Grand Strategy in der östlichen arabischen Welt widersprochen. SECORD, Honored and Betrayed, S. 44-51, später Mitverschwörer in der IranContra-Affäre der 1980er Jahre, schildert seine Teilnahme an einer geheimen Unterstützungsmission für die iranische Armee im Kampf gegen Aufständische in der Kurdenregion und Bārzāni vom März 1963 bis September 1964 mit 80 amerikanischen Special Forces, hier offenbar noch ohne grenzüberschreitende Aktionen im Irak. Die ausgegebene Devise im Weißen Haus war, jeden Kontakt zu den Kurden zu vermeiden. Vgl. Saunders Memo, 29.4.65, ,Iran, Vol. I, Cables 1/64-12/65', NSF, Country File, Box 136, LBJL; die Israelis versuchten die Administration von den Vorzügen einer verdeckten Operation zu überzeugen. Vgl. Symmes Letter, 8.11.65, ,Political Affairs \& Rel. Iran/Israel 1965‘, Lot 69 D 482, NEA/IRAN: 1964-66, Box 9.

39 Die Iraker hatten sich intern bereit erklärt, eine Kompromisslösung in der Šațț al-'Arab-Frage anzustreben, solange keine formale Aufgabe von Territorium verlangt werde. Im Gegenzug verlangten sie iranische Verpflichtung auf ,non-interference“, also Aufgabe der Unterstützung für die irakischen Kurden, was Teheran ablehnte. Vgl. Tehran \#A-532, 19.4.65, POL IRAN-IRAQ; Tehran \#A-762, 14.5.66, POL 1 IRAN-IRAQ, CF 6466.

${ }^{40} \mathrm{Vgl}$. Baghdad \#A-172, 11.8.65, POL IRAN-IRAQ, ebd.
} 
militärisch auf die Rückzugsgebiete der aufständischen Kurden auf iranischem Territorium und den Waffenschmuggel zielend. Iran drohte daraufhin mit militärischer Vergeltung und der Aufkündigung des Grenzvertrags von 1937. Zwar beruhigte sich die militärische Lage wieder etwas im Laufe des Jahres 1966 und im Anschluss an den Tod 'Abd as-Salām 'Ārifs in einem Flugzeugabsturz im April und der Übernahme der Präsidentschaft durch 'Abd al-Raḥman 'Ārif, der sich stärker als sein Bruder von der VAR distanzierte. ${ }^{41}$ Im Kern aber blieben zentrale Fragen im bilateralen Verhältnis zwischen beiden Staaten ungelöst und die zum Jahreswechsel 1965/66 einsetzende militärische Konfrontation setzte sich in den Folgejahren fort. ${ }^{42}$

Insgesamt ergab sich aus amerikanischer Sicht eine bedenkliche Entwicklung der iranischen Außenpolitik, die stark reaktiven Charakter auf einen doch eher imaginären „Arab Threat“ angenommen hatte. Mit seiner Politik trug Mohammad Rezā aktiv zur weiteren Polarisierung in der Region bei, eine aus amerikanischer Perspektive nicht wünschenswerte Entwicklung. Die CIA warnte im Mai 1966:

In responding to the Arab 'threat,' the Shah has followed a policy of supporting governments, elements, and activities which might keep his enemies distracted and occupied. The Iranian Government has directed a hostile - sometimes shrill - propaganda campaign against Nasir, both in Iran and abroad. Iran has given military equipment and financial assistance to the Kurdish rebellion, which keeps a large percentage of Iraqi troops tied up in northern Iraq. [31/2 lines of source text not declassified] The Iranians have attempted openly to win support among leaders of the Shi'a minority Islamic group in Iraq. Iran has purchased arms for Saudi Arabia to pass on to the Yemeni royalist forces, and has maintained close liaison with Nasir's primary enemy, Israel. Among the more conservatively ruled Arab states, the Shah has attempted particularly to woo Saudi Arabia and Jordan and has given sympathetic attention to Saudi King Faisal's proposal, which Nasir opposes, regarding Islamic solidarity. ${ }^{43}$

Angesichts der iranischen Politik, ähnlich wie oben geschildert für das Jahr 1963, gab es auch jetzt wieder interne Debatten über die angemessene Haltung der US-Administration. Inzwischen aber war die „Unabhängige Nationale Politik“ Mohammad Rezās etablierte Realität und die Vorstellung, man könne dem Verbündeten eine bestimme Außenpolitik aufoktroyieren, war angesichts des eigenen Einflussverlusts, der sich nun auch im letzten verbliebenen Bereich des Militärischen ankündigte, kaum noch verbreitet. ${ }^{44}$ Prinzipiell war eine unabhängige und unbeeinflusste Außenpolitik Irans durchaus mit der amerikanischen Grand Strategy in der Nahostregion vereinbar. Langfristig problematisch aber wurde die zunehmende Eigenmächtigkeit der bisherigen regionalen Klienten in Kombination mit der seitens

\footnotetext{
${ }^{41}$ Vgl. Bevans Memo, 15.2.66, ,POL IRAN/IRAQ (Jan. Feb.) IRAN 1966', Lot 70 D 330, NEA/IRAN: 1964-66, Box 17; vgl. Iran-Iraq Chronology, o.D., ebd.; zur Entspannung ab 1966 vgl. Tehran \#A-31, 18.7.66; Tehran \#A319, 4.11.66, POL IRAN-IRAQ, CF 64-66; vgl. MemCon, 8.10.65, ,Iraq, Volume 1, 12/63-7/68', NSF, Country File, Box 138, LBJL.

${ }^{42}$ NEA identifizierte die Spannungen zwischen Iran und den arabischen Nachbarstaaten als ein für die Zukunft womöglich zentrales Konfliktfeld und forderte INR zu einer umfassenden nachrichtendienstlichen Analyse des Gesamtzusammenhangs auf. Vgl. Talbot Memo, 13.7.64, ,Office Memoranda', Lot 69 D 30, NEA/IRN: 1964-66, Box 1.

${ }^{43}$ CIA Intel Memo No. 1355/66, 21.5.66, FRUS 64-68, XXII., S. $248 f$.

${ }^{44} \mathrm{Vgl}$. Symmes Memo 28.12.65, ,Political Affairs \& Rel. IRAN/IRAQ IRAN 1965', Lot 69 D 482, NEA/IRAN: 1964-66, Box 9.
} 
Washington nachdrücklich unterstützten Aufrüstung dieser Staaten (Iran, Israel, SaudiArabien), teilweise mit militärischer Hochtechnologie amerikanischer Provenienz bzw. im Falle Israels direkt und indirekt finanziert vom amerikanischen Steuerzahler. Somit trug die amerikanische Politik selbst zur Aufhebung der innerregionalen Machtbalance und damit zur Unterwanderung der eigenen Gesamtstrategie in der Region bei.

Angesichts der fortgesetzten Eskalation in den iranisch-irakischen Beziehungen war der sich abzeichnende Schwenk in der amerikanischen Haltung bezüglich des „Arab Threat“ bedenklich. Lange von US-Seite als nicht ernstzunehmende Übertreibung bzw. als Vorwand für neue Aufrüstungsforderungen behandelt, setzte sich erstmals im Zuge der militärischen Erkundungskommission vom Frühjahr 1966 eine teilweise Übernahme der iranischen Bedrohungsvorstellungen durch amerikanische Planer durch. So schlussfolgerte der PetersonBericht aus einseitig militärischer Perspektive: ,The combined forces of Syria, the UAR, and Iraq represent an overwhelming military capability vis-à-vis Iran.“ Zugleich bestätigte der Bericht die iranische Behauptung, die eigene „vital oil lifeline is exposed““45 Fast zeitgleich bewertete die CIA die Bedrohung: In der Tat könnten Nassers Bemühungen, durch Subversion und Propaganda ägyptischen Einfluss auf die Golfaraber für die Zeit nach dem britischen Rückzug zu gewinnen, ,[...] with some validity as posing a long-range threat to Iranian transit through the Strait of Hormuz" konstruiert werden Insgesamt aber sei die Bedrohung vorerst eher ,at most only potential, and indirect at present“. ${ }^{46}$ Insgesamt aber zeichnete sich eine größere Identifikation mit den Bedrohungsvorstellungen Mohammad Rezās auch auf amerikanischer Seite, zuvorderst aufseiten des Pentagons und der Nachrichtendienste ab. ${ }^{47}$

Die Problematik, die eigene regionale Ordnungspolitik, für die Supermächte immer basierend auf und abhängig von globalen Interessen, mit den Partikularinteressen der Klientelstaaten bzw. später der zunehmend unabhängigen Verbündeten in Einklang zu bringen, zeigte sich auch beispielhaft in Südasien, an der Entwicklung des pakistanisch-amerikanischen Verhältnisses, auch hier wieder mit direkten Auswirkungen auf die zukünftige Politik gegenüber Iran. Ayub Khan, seit 1958 Militärherrscher, versuchte 1965 durch militärischen Aktivismus eine für Pakistan günstige endgültige Lösung der Kaschmirfrage herbeizuführen. Nach der durch britische Vermittlung entschärften sogenannten Rann of Kutch-Krise im Frühling und Sommer 1965 eskalierte Pakistan den Konflikt erneut durch massive Infiltration von „Freischärlern“ jenseits der Line of Control. Im Laufe des Septembers 1965 führte dies zu einem konventionellen Krieg zwischen den beiden südasiatischen Mächten, aus dem Indien als

\footnotetext{
${ }^{45}$ Tehran \#A-738, 7.5.66, DEF 19 US-IRAN, CF 64-66; die DIA hatte noch im Januar 1966 in einer eingehenden Untersuchung des "Non-Soviet Military Threat to Iran“ beschwichtigt. Ein Einsatz des militärischen Gesamtpotentials der arabischen Staaten gegen Iran ,en toto against Iran is unlikely in the next five years in view of inter-Arab rifts, Arab fears of an Israeli preemptive attack, and of current Arab commitments in Yemen and against the Kurds. Neither the Iraqi ground forces nor the estimated projected improvement of the Iraq Air Force pose a very formidable threat to Iran."; Special DIA Intelligence Estimate, 28.1.66, FRUS 64-68, XXII, S. 207; allerdings bestätigte auch die DIA die theoretische Verwundbarkeit des ressourcenreichen Südwestens.

${ }^{46}$ CIA Intel Memo No. 1355/66, 21.5.66, ebd., S. 248.

${ }^{47}$ Vgl. [Near East-South Asia IRG Meeting, 12.5.66, ebd., S. 241-4.
} 
militärischer Sieger hervorging, wenngleich der Status Quo im Kern unverändert blieb. ${ }^{48}$

Die USA hatten auf den Zweiten Indisch-Pakistanischen Krieg und die Benutzung von amerikanischen Militärgütern, die im Rahmen des MAP-Programms geliefert worden waren, mit einem Waffenembargo gegen die kriegführenden Staaten reagiert, was angesichts der fast ausschließlichen Ausrüstung Pakistans mit US-Systemen Rawalpindi eindeutig benachteiligte. ${ }^{49}$ Ayub Khan wandte sich sogleich an seine CENTO-Verbündeten Iran und die Türkei, aber auch an Saudi-Arabien, um Hilfe. Ankara und Teheran brandmarkten Indien öffentlich als Aggressor - die USA aber untersagten Lieferungen amerikanischer Militärgüter und selbst von Munition von Iran nach Pakistan, trotz inständiger Anfragen des Schahs und des Ministerpräsidenten Howejdā und der expliziten Warnung, dass bei Ausbleiben von Unterstützung die Auflösung des CENTO-Bündnisses unausweichlich sei. ${ }^{50}$

Selbst die per Kredit jüngst erworbenen Waffen, obgleich mit iranischem Kapital bezahlt, unterlagen den Beschränkungen der Weitergabe an Drittländer, wie alle übrigen MAP-Güter auch. Der Schah sei ,[...] particularly bitter over thought that he is not $\{$ the; R.P. $\}$ full master of his military establishment. “"51 Seinem Ministerpräsidenten gegenüber brachte es Mohammad Rezā auf den Punkt: „We are not free.“52 In der Folgezeit sollte Mohammad Rezā den pakistanischen Fall immer wieder als Beispiel und Begründung für seine verstärkten Bemühungen anführen, sich von der einseitigen Abhängigkeit von den USA im militärischen Feld zu lösen. ${ }^{53}$ Der Botschaft in Teheran zufolge hatte der Waffengang mittelfristig sogar größere Auswirkungen auf das Verhältnis zu den USA als auf Irans Beziehungen zu den beiden kriegführenden Mächten. Ähnlich wie Pakistan konzentriere sich nun auch Iran auf die regionale Bedrohungslage, zuvorderst den „Arab Threat“. Da sich die CENTO allein auf die

\footnotetext{
${ }^{48}$ Für beispielhafte Erörterung der strategischen Folgen des Konflikts für die USA vgl. Rusk Memo, 9.9.65; Bundy/Komer Memo, 5.10.65, FRUS 64-68, XXV, S. 375ff., 444-7; Komer kritisierte die Stagnation in der amerikanischen Südasienpolitik und plädierte für einen neuen Ansatz. Er stimme McNamara zu, dass Südasien ,just as central as Southeast Asia“ sei. Gegenüber LBJ wurde Komer noch expliziter: „,...] nobody needs to tell you that the Indian subcontinent is still the key area we, Moscow, and Peiping are all competing for in Asia, and to which Vietnam is only an antechamber"; Komer Memo, 30.5.65; Komer Memo, 10.6.65, ,Komer Memos, Volume I [2], [1 of 2]'; Komer Memo, 31.8.65, NSF, Name File, Box 6 [1 of 2]; offenbar hatte Ayub Khan nach Informationen der CIA bereits im Juli 1964 kurzzeitig den Austritt aus CENTO beschlossen, die Entscheidung aber kurz darauf revidiert. vgl. CIA, Intelligence Information Cable, 13.7.64, ,Pakistan, Vol. II, Cables, 6/64 + $1 / 64^{\prime}$, NSF, Country File, Box 151, LBJL.

${ }^{49}$ Vgl. Rawalpindi \#52, 10.9.65, FRUS 64-68, XXV, S. 385-8.

${ }^{50}$ Vgl. ebd., S. 384 n5; die Iraner vertraten die Auffassung, die pakistanischen Aktionen in Kaschmir hätten in umstrittenen Gebiet stattgefunden, der indische Gegenschlag über die anerkannte Grenze sei dagegen als Aggression zu klassifizieren. Vgl. Tehran \#291, \#294, \#345, 6./7./12.9.65; State \#303, 13.9.65, , Iran Cables, Vol. I, 1/64-12/65', NSF, Country File, Box 136, LBJL; der Schah hatte auch heimliche Lieferungen ins Spiel gebracht, um auf diese Weise die USA von jeder Verantwortung zu befreien, was von amerikanischer Seite ebenfalls abgelehnt wurde. Offenbar war der Schah sogar zu einem begrenzten Einsatz iranischer Truppen in Pakistan bereit, vorwiegend zur Bewachung strategischer Punkte im Hinterland, mit der Absicht, pakistanische Kräfte für die Front freizusetzen. Vgl. Tehran \#337, 11.9.65, ebd.; die CIA bestätigte, dass ein pakistanischer Rückzug ,[...] would just about finish the weak CENTO organization"; vgl. CIA Office of Current Intelligence Study, 15.9.65, ,Pakistan, Vol. IV, Memos 8/65-9/65', NSF, Country File, Box 151, LBJL; Iran und Pakistan hatten bereits Ende 1964 eine neue Kooperation im gemeinsamen Kampf gegen eine in Entstehung begriffene belutschische Freiheitsbewegung aufgenommen. Vgl. Karachi \#744, 10.10.64, POL 23-9 PAK, CF 64-66.

${ }^{51}$ Tehran \#337, 11.9.65, ,Iran Cables, Vol. I, 1/64-12/65', NSF, Country File, Box 136, LBJL.

${ }_{52}$ Tehran \#469, 24.9.65, FRUS 64-68, XXII, S. 181; vgl. Tehran \#A-231, 30.9.65, POL IRAN-US, CF $64-66$.

${ }^{53}$ Vgl. Draft Telegram, o.D., encl. to Meyer Letter, 11.9.65, ,POL Iran/US 1965', Lot 69 D 490, NEA/IRN: 196466 , Box 12.
} 
Abwehr der sowjetischen Gefahr fokussierte, sei sie im iranischen strategischen Denken in ihrer Bedeutung noch weiter abgewertet worden. Der Schah bezeichnete das Bündnis im Gespräch mit Meyer als „Maskerade“ und „im Sterben liegend“. Wenn überhaupt noch kollektive Verteidigung ernsthaft in Erwägung ziehend, so präferierte er nun die intensivierte Zusammenarbeit zwischen den regionalen CENTO-Mitgliedern im Rahmen des 1964 gegründeten RCD (Regional Cooperation for Development), der aber bislang ausschließlich auf wirtschaftlichem Gebiet operierte. ${ }^{54}$ Allerdings registrierte die US-Botschaft auch einen positiven Effekt des Kriegs auf die bilateralen Beziehungen. Bei aller verstärkten Tendenz hin zu mehr Unabhängigkeit diente das Schicksal Pakistans zugleich als Warnung für die Folgen einer Balance-Politik zwischen den Supermächten. Trotz der Annäherung an die Volksrepublik China hatte Ayub keine nennenswerte Unterstützung vonseiten Beijings erhalten - andererseits aber hatte die Distanzierung von Washington eine wichtige Rolle in der militärischen Niederlage gespielt, nicht zuletzt sogar die Unterstützung durch regionale Verbündete Rawalpindis unterbindend. Offenkundig seien die ,monumental risks in trying to play big powers against each other". 55

Wie bereits im vorherigen Unterkapitel aufgezeigt, verstärkte der Zweite Indisch-Pakistanische Krieg die Entschlossenheit Teherans, auch im militärischen Feld größtmögliche Souveränität zu erlangen, dabei eine Krise in den Beziehungen mit Washington bewusst in Kauf nehmend. Der zwischenzeitlich befürchtete Kollaps des CENTO-Bündnisses trat allerdings nicht ein. Zudem konsultierten Iran und die USA während der Krise regelmäßig und stimmten ihr jeweiliges Vorgehen aufeinander ab, auf diese Weise die aus dem Konflikt entstandenen Belastungen des gemeinsamen Verhältnisses zumindest in Teilen überspielend. ${ }^{56}$ Einige zusätzliche Probleme ergaben sich allerdings nach dem Waffenstillstand. Nachdem Teheran sich dem seitens der USA durchgesetzten Waffenembargo während des Kriegs gebeugt hatte,

\footnotetext{
${ }^{54}$ Der RCD war von den regionalen CENTO-Mitgliedern im Juli 1964 gegründet worden mit dem Ziel der Förderung der wirtschaftlichen, technischen und kulturellen Kooperation zwischen den drei Staaten. Die Gründung spiegelte in erster Linie die vorwiegend politische Frustration der Türkei und Pakistans mit dem Westen im Kontext der Zypern- und Kaschmirfragen wider. In gewisser Weise war die Gründung des RCD ein Kompromiss - Pakistan dachte offenbar zwischenzeitlich an die Formierung eines neutralistischen Blockes mit den regionalen CENTO-Verbündeten. Vgl. CIA Intelligence Information Cable, 13.7.64, ,Pakistan, Vol. II, Cables, 6/64 + 1/64‘, NSF, Country File, Box 151, LBJL; gedacht als erster Schritt hin zu regionaler Integration, mit Beitrittsoption für Nachbarstaaten wie Afghanistan, blieb der RCD bis zur Iranischen Revolution letztlich zweitrangig. Vgl. Policy Planning Council Study, 30.6.66, encl. to Owen Memo, 23.7.66, ,Near and Middle East 1965-66“, Lot 72 D 139 , S/P Records, Subject and Country: 1965-69, Box 315; Tehran \#A-305, 28.10.65, POL Iran-PAK, CF 64-66; gegen den langfristigen Erfolg sprach, dass die Volkswirtschaften der drei Staaten wenig komplementär waren - der Handel mit den jeweiligen Anderen übertraf in keinem der drei Staaten mehr als $10 \%$ des Gesamtvolumens. Für die Umstände der Gründung des RCD und seine Strukturen und Funktionen vgl. YeSILBURSA, Formation of RCD; vgl. auch HALE/BHARIER, R.C.D.; GÖKTEPE, Forgotten Alliance, S. 120-6; für die Ảngste bezüglich CENTO vgl. auch State \#5386, 26.2.65, FRUS 64-68, XXII, S. 131; vgl. POOLE, History of the JCS: IX, S. $189 \mathrm{ff}$.

${ }_{55}$ Tehran \#A-231, 30.9.65, POL IRAN-US, CF 64-66.

${ }^{56}$ Vgl. Johnson Letter to Shah, 5.10.65, FRUS 64-68, XXII, S. 182f.; aus amerikanischer Sicht hatte man ohnehin dem bilateralen Abkommen vom März 1959 immer größere Bedeutung für die strategische Kooperation zwischen beiden Staaten zugeschrieben. Umso überraschter war die Administration, als der Schah sich auch über die 1959erVereinbarung unzufrieden zeigte. Offenbar interpretierte Mohammad Rezā die im Abkommen enthaltene Beistandsverpflichtung ausschließlich auf Aggression seitens der UdSSR gerichtet. Eine Studie des Legal Adviser bestätigte im Kern diese Sichtweise. Vgl. Herz Letter, 27.10.66, ,POL IRAN/U.S. 1966', Lot 70 D 330, NEA/IRN: 1964-66, Box 17; die Briten teilten die Einschätzung über den geringen strategischen Nutzen der CENTO, doch würde der Schah eine Auflösung als einen „Sieg Nassers“ betrachten; siehe Record of Conversation [Hare, Roger Allen u.a.], 4.2.66, FO 371/186422, TNA:PRO.
} 
unterstützte es im Anschluss Pakistan direkt bei seinen Wiederaufrüstungsbemühungen und fungierte für die Pakistaner zeitweilig als Zwischenhändler. So erwarb man offenkundig im pakistanischen Auftrag 90 außer Dienst gestellte F-86-Düsenjäger aus der Bundesrepublik Deutschland. Nach der Auslieferung der ersten Flugzeuge gaben die Iraner gegenüber amerikanischen Gesprächspartner offen zu, diese seien für Pakistan bestimmt. Die iranische Rolle sorgte dabei für einige diplomatische Verstimmung ${ }^{57}$ Auch in den Folgejahren agierten die Iraner als Zwischenhändler für Pakistan, z.B. erwarben sie Ersatzteile für amerikanische Waffensysteme im Auftrag Rawalpindis. ${ }^{58}$ Andererseits vermittelte der Schah weiterhin aktiv gegenüber Ayub Khan und versuchte diesen, von einer noch stärkeren Hinwendung zu Beijing abzuhalten. ${ }^{59}$

Abseits der Verstimmungen infolge des amerikanischen Vetos gegen direkte Militärhilfe an Pakistan hatte der Zweite Indisch-Pakistanische Krieg allerdings auch einige positive Folgen für das bilaterale Verhältnis. Die sich aus dem Krieg ergebenden weiteren Belastungen für das Verhältnis zwischen Rawalpindi und Washington wurden, zumindest in Teilen, durch kompensatorische Aufwertungen des amerikanisch-iranischen Verhältnisses aufgefangen ${ }^{60} \mathrm{Im}$ Zuge der Verschlechterung der pakistanisch-amerikanischen Beziehungen hatte sich eine Ablösung der bisherigen engen geheimdienstlichen Zusammenarbeit abgezeichnet, die im Angesicht zunehmender chinesischer Penetration des Landes kaum noch aufrechtzuerhalten war. ${ }^{61}$ Ayub Khan reagierte auf die amerikanische Haltung während des Kriegs gegen Indien mit einer Distanzierung vom Verbündeten. Nachdem die Pakistaner im September 1965 begannen, die Geheimdienstoperationen auf pakistanischem Territorium zu behindern, bereitete die Johnson-Administration die Verlegung der sensiblen Anlagen nach Iran vor. ${ }^{62}$ Botschafter Meyer warnte vor einer weiteren Ausdehnung der bereits beachtlichen amerikanischen Geheimdienstpräsenz in Iran, gerade angesichts des allgemeinen Trends in den nicht-westlichen Staaten hin zu einer nationalistischen Einstellung und der Betonung der eigenen Souveränität, ein Trend, der gerade auch in Iran immer spürbarer werde. Sollte die geheimdienstliche Präsenz und die „burgeoning US official presence“ - zum damaligen Zeitpunkt bereits 1.500 Amerikaner in unterschiedlichsten Funktionen plus Familienanhang -

\footnotetext{
$\overline{57}$ Vgl. Mudd Letter, 4.8.66, DEF 12-5 IRAN, CF 64-66.

${ }^{58}$ Letztlich war man sich auch im State Department bewusst, dass das Embargo angesichts zunehmender indischer Waffenkäufe von der Sowjetunion nicht realistisch war. Vgl. Mudd Letter, 10.12.66, ,DEF 19-3 Third Country Sales Iran 1966“; Thacher Letter, 8.9.66, ,DEF 19-3 Military Assistance Iran 1967‘, Lot 70 D 330, NEA/IRN:196466, Box 18.

${ }_{59}$ Vgl. State Telegram \#672 to Tehran, 8.12.65, ,Iran, Vol. I, Cables, 1/64-12/65', NSF, Country File, Box 136, LBJL.

${ }^{60}$ Die stark negativen strategischen Folgen des Kriegs von 1965 für die US-Position in Südasien konnten allerdings nicht auf diese Weise kompensiert werden. Vgl. Komer Memo, 5.10.65, ,Komer Memos, Vol. 2 [2] $]^{`}$, NSF, Name File, Box 6 [1 of 2], LBJL.

${ }^{61}$ Das 303 Committee hatte bereits im August 1965 die Einstellung der Bauarbeiten an neuen Abhör- und Messstationen in Peschawar erörtert, um den Druck auf Ayub Khan zu erhöhen. Bereits zu diesem Zeitpunkt war Iran als alternativer Standort ins Auge gefasst worden. Vgl. Bundy Memo, 6.7.65; Meeting Minutes 303 Committee, 9.8.65; Bundy Memo [NSAM N ${ }^{\circ} 337$, ,US Intelligence Facilities in Pakistan'], 10.8.65 FRUS 64-68, XXV, S. 293f., 330ff., 333.

${ }^{62}$ Vgl. Komer Memos, 29.9./1.10.65, ebd., S. 434ff., $437 \mathrm{ff}$.; der Druck auf die Abhöreinrichtungen war Teil einer größeren pakistanischen Kampagne gegen die US-Präsenz.
} 
von der Opposition zu einer nationalistischen Frage aufgebauscht werden und der Schah zum Entgegenkommen gezwungen sein, drohe vielleicht sogar der ,ouster of \{the; R.P. $\}$ great bulk of $\{$ the $\}$ American presence here, including our existing sensitive operations. “63

Entgegen Meyers Bedenken forcierte die Johnson-Administration die Übersiedlung der Abhörstationen von Pakistan nach Iran. ${ }^{64}$ Bereits im September 1965 wurde ein Erkundungsteam nach Iran entsandt, das die örtlichen Gegebenheiten für einen Ausbau der geheimdienstlichen Präsenz begutachtete. ${ }^{65}$ Im Mai 1966 genehmigte Präsident Johnson schließlich den Ausbau bestehender Stationen in Iran, vorerst um für eine später notwendig werdende Verlagerung der pakistanischen Einrichtungen gewappnet zu sein - ein Teil der amerikanischen Kompromissbereitschaft in den Verhandlungen über die nächste Militärkredittranche in dieser Zeit wie z.B. die Senkung der verlangten Zinsrate für den Kredit war auf die zeitgleich laufenden Verhandlungen mit den Iranern über die neuen, intelligence facilities" zurückzuführen. ${ }^{66}$ Zudem gab es offenbar eine Geheimabsprache mit dem Schah, für Iran relevante Informationen, die mit den neuen Anlagen gewonnen würden, den iranischen Diensten zugänglich zu machen. ${ }^{67}$ Im Mai 1968 forderte die pakistanische Regierung letztendlich die Schließung Peschawars nach Ablauf des zugrunde liegenden Abkommens zum Juli 1969 - durch die Verlegung wichtiger zusätzlicher Stationen wurde Iran im Anschluss mit seinen umfangreichen amerikanischen Abhör- und Messstationen zu dem für die Beschaffung geheimdienstlich relevanter Informationen mit Abstand wichtigsten Staat entlang der sowjetischen Peripherie. ${ }^{68}$ Meyer zufolge war die im kaiserlichen Jagdreservat entstehende, \$5 Millionen teure Horchstation ,[...] a most impressive one and when completed will make Tehran equal in importance to almost any other site of similar facilities in the world." ${ }^{69}$ Die ausgedehnte Präsenz amerikanischer Stationen bildete nach dem Ende von Wirtschafts- und

\footnotetext{
${ }^{63}$ Tehran \#332, 10.9.65, FRUS 64-68, XXII, S. 173; Meyer glaubte zudem, dass die Vorteile aufgrund geographischer Gegebenheiten in Nordpakistan in Iran nicht gegeben seien und eine erhebliche Ausweitung der bestehenden Einrichtungen angesichts der politischen Gefahren somit unnötig sei: „Since we already have well established facilities [1 line of source text not declassified], I think it would be most unwise to tempt fate by importing additional hundreds of Americans. Camel's back is already heavy laden, from standpoint of what is politically tolerable here. Why risk destroying extensive facilities already in being for minimal additional product?"; ebd.; zur amerikanischen Präsenz vgl. Tehran \#229, 28.8.65, ebd., S. 167.

${ }^{64}$ Vgl. State \#292, 11.9.65, ebd., S. $174 \mathrm{f}$.

${ }^{65}$ Komer instruierte das State Department, die Verlegung der Abhörstationen habe nunmehr ,super-priority“. Vgl. State \#345, 17.9.65, , Iran, Cables, Vol. I, 1/64-12/65'; Spain Memo, 20.9.65, Iran, Memos\& Misc, Vol. I, 1/64$12 / 65^{\circ}$, NSF, Country File, Box 136, LBJL.

${ }^{66}$ Vgl. Rostow Memo, 27.5.66, FRUS 64-68, XXII, S. 260f.; die eigentliche Instruktion Johnsons in NSAM N³48, ,Alternatives to US Facilities in Pakistan', 30.5.66, o.F., NSAM, Box 4, RG 273, NACP; offenbar hatte Komer ein ,Alternativpaket“ im Falle einer Schließung der Anlage in Pakistan vorbereitet, das im Falle der Verlegung nach Iran im Gegenzug die Senkung der Zinsrate für die nächste Tranche des Militärkredits von 5\% auf 3,5-4\% vorsah. Vgl. Komer Memos, 13.11.65/18.12.65, ,Komer, Memos, Volume 2 [1] [1 of 2]', NSF, Name File, Box 6 [1 of 2], LBJL; der widerspenstige Meyer hatte im Gegenzug ein umfangreiches Entgegenkommen hinsichtlich der meisten bilateralen Fragen gefordert, neben Militärkredit und PL-480-Vereinbarungen auch die (verfassungsrechtlich kaum umsetzbare) Suspendierung des sogenannten Gudarzijān-Falles durch Intervention der US-Regierung und harte Maßnahmen gegen iranische oppositionelle Studenten in den USA. Vgl. Tehran \#355, 13.9.65, FRUS 64-68, XXII, S. 175-9; ein anderes Memo schlug den Abschluss einer mehrjährigen PL-480Vereinbarung vor. Die Forderungen bezüglich Gudarzijān seien dagegen „legally impossible“; Handley Memo, 8.11.65, ,AID 15-11 [...]', Lot 69 D 426, NEA/IRN: 1964-66, Box 8.

${ }^{67}$ Vgl. Gratian Yatsevitch OHI, 5.11.88, OHIC, S. I-123.

68 Vgl. Katzenbach Memo, 5.7.68, FRUS 64-68, XXV, S. 988-91; Rusk Memo, 7.6.68, FRUS 64-68, XXII, S. 521f.; Tehran \#05852, 27.7.68, ,Iran Cables, Vol. II, [1 of 2]; 1/66-1/69‘, NSF, Country File, Box 136, LBJL.

${ }^{69}$ Meyer Letter, 19.11.66, DEF 19-8 US-IRAN, CF 64-66.
} 
Finanzhilfe, der Auflösung der US-Involvierung in die Wirtschaftsplanung und der Erosion des Militärmonopols einen neuen Stützpfeiler der besonderen Beziehungen zwischen beiden Staaten - allerdings stärkte die enge Zusammenarbeit eher die Hebelkraft Mohammad Rezās gegenüber Washington als umgekehrt. ${ }^{70}$

Insgesamt ergab sich aus der neuen unabhängigen Außenpolitik aus Sicht Washingtons ein zunehmend zwiespältiges Bild. Grundsätzlich positiv aus Sicht der amerikanischen Administration war die Entspannung zwischen Iran und der UdSSR. Zwar blieb viel Misstrauen über die letztendlichen sowjetischen Absichten, doch überwog auf US-Seite die Erleichterung, dass die Befürchtung einer sowjetischen Übernahme Irans, maßgeblich bestimmend für die eigene Politik seit 1946, als eine nunmehr kaum noch ernstzunehmende Bedrohung erschien, ja von Moskau selbst nicht mehr aktiv betrieben wurde, von den weiterhin aktiven Propagandasendern einmal abgesehen. Somit konnte man in Washington auch akzeptieren, dass sich Iran in vielerlei Hinsicht den Positionen der nichtpaktgebundenen Staaten annäherte. ${ }^{71}$ Die sich aus der neuen unabhängigen Positionierung Irans in der Staatenwelt ergebende größere Popularität des Schahs im Innern und die damit einhergehende Stärkung der Stabilität war aus US-Perspektive ebenfalls höchst willkommen. ${ }^{72}$ Gleichzeitig ergaben sich aus der Unabhängigen Nationalen Politik auf dem internationalen Feld auch zentrale neue Probleme für die amerikanische Iranpolitik, wenngleich die meisten Beobachter diese vorerst nicht wahrnahmen und stattdessen das offizielle Narrativ über den beispiellosen Erfolg des nation-building der USA in Iran weitgehend kritiklos akzeptierten. Erstens ergab sich aus der Tragweite der iranischen Ambitionen eine Notwendigkeit stark beschleunigter Aufrüstung, die letztlich zu Lasten der ökonomischen Entwicklung und des inneren Reformprogramms ging, wenngleich der rasche Anstieg der Öleinnahmen zumindest übergangsweise eine „guns AND butter"- Strategie zuließ. Das zunehmend gigantische Rüstungsprogramm retardierte nicht nur die innere Entwicklung, sondern führte zu den bereits geschilderten diplomatischen Spannungen mit dem bisherigen Hauptlieferanten im militärischen Bereich, den USA. Gleichzeitig erforderten die Waffenkäufe das Erschließen neuer Einnahmequellen, was in erster Linie in steigendem Druck Irans auf das Ölkonsortium Ausdruck fand, eine aus Sicht Washington ebenfalls alles andere als willkommene Entwicklung. Zweitens beseitigte die neue Handlungsfreiheit früheren amerikanischen Einfluss auf die iranische Außenpolitik. Obgleich

\footnotetext{
${ }^{70}$ Im April 1970 sprach sich DCI Richard Helms für den Aufbau einer „special relationship“ mit Iran aus, allein auf der Grundlage der außerordentlichen Bedeutung der Mess- und Horchstationen. Siehe Memo for the Record, 22.10.69; Helms Memo, 16.4.70, part of Saunders Memo to Kissinger, 16.4.70, FRUS 69-76, E-4, docs. 31, 63; zur Bedeutung gerade der beiden sog. Tacksman-Stationen vgl. DAUGHERTY, Shadow, S. 19ff., $31 \mathrm{f}$.

${ }^{71}$ Vgl. Tehran \#A-51, 1.8.66, POL 1 IRAN, CF 64-66; ab 1966 deuteten sich Ambitionen an, im Rahmen einer „dritten Kraft“" zu einem der Fürsprecher der Entwicklungsstaaten zu werden, ein erstes Anzeichen für die späteren globalen Ambitionen des Schahs. Für die Tendenz hin zur Weltsicht der Nichtpaktgebundenen vgl. auch ZABIH, De Facto Nonalignment.

${ }^{72}$ In den anglo-amerikanischen Nahostkonsultationen Anfang 1964 hielt ein US-Positionspapier demgemäß fest, ,we are not concerned about the new departures in Iranian foreign policy and we recognize the considerable domestic political benefits of the current show of independence"; Position Paper, , The Iranian Situation and Outlook', 22.1.64, ,U.S.-U.K. Talks-25-30 Jan. 1964‘, Lot 67 D 495, Subject Files, 1962-1966, G/PM, Office of Operations, Box 3.
} 
nicht mehr in der Lage, Teherans Politik mitzubestimmen, wurde der bisherige Patron dennoch weiterhin für die iranischen Handlungen verantwortlich gemacht. Für Außenstehende erschien die weiterhin enge militärische und geheimdienstliche Kooperation zwischen beiden Ländern als Beleg für ein substantielles Weiterbestehen der Allianz in ihrer bisherigen Form und ein Fortdauern amerikanischen Steuerungsvermögens gegenüber Iran. In einer konsequenten und, zugegeben, idealtypischen Umsetzung einer Offshore-Balancing-Strategie, hätten sich die USA auch aus den verbliebenen Bestandteilen der früheren Allianz im Sinne eines De-Alignment konsequent zurückgezogen und nötigenfalls sogar aktiv Gegenmachtbildung in der Golfregion gegen iranische Vormachtsbestrebungen unterstützt. Vor allem die Beharrungskraft der bisherigen Politik ${ }^{73}$, aber auch die Unfähigkeit, sich eine freiwillige Aufgabe der bisherigen Monopolstellung überhaupt vorzustellen, und die Überzeugung nun die Ernte für die Stabilisierungspolitik der vergangenen Jahrzehnte einzufahren, aber auch handfeste finanzielle und wirtschaftliche Interessen, machten eine solche Politik bereits im Ansatz undenkbar. Die USA waren nun an die Außenpolitik des Schahs gebunden, eine Entwicklung, die sich mit der späteren Politik der Nixon-Administration noch verschärfen sollte. Während der Beratungen in IRG/NEA im Mai 1966 brachte James H. Critchfield, der Chief der Near East and South Asia Division in der CIA, erstmals die Idee auf, den Vorschlag Mohammad Rezās, als Repräsentant westlicher Interessen in der Region zu agieren, aufzugreifen, ein früher Verweis auf den später mit der Nixon-Doktrin verfolgten Ansatz, ordnungs- und sicherheitspolitische Aufgaben an die pro-westlichen Regionalmächte zu übertragen. ${ }^{74}$ Derartige Überlegungen waren durchaus vereinbar mit Mohammad Rezās langfristigen Vorstellungen, Iran in eine „command position“ in der Region zu versetzen. ${ }^{75}$

Vorerst wurde die oberflächlich pro-westliche Politik Mohammad Rezās als willkommener Gegensatz zu der zunehmend feindseligen Einstellung der meisten arabischen Staaten im Nachgang des Dritten Arabisch-Israelischen Krieges im Juni 1967 wahrgenommen, zumal der Schah mit seiner unabhängigen Ölpolitik zusätzlich an Gewicht gewinnen konnte. Hier und bei anderen Gelegenheiten glich der Schah den Eindruck einer unter Umständen zu eigenständigen oder sogar pro-sowjetischen Orientierung aus. Ähnlich intendiert war auch die offizielle Unterstützung der amerikanischen Militärintervention in Südvietnam - als einer von wenigen US-Verbündeten beteiligte sich Iran aktiv, wenn auch nur mit der symbolischen Entsendung einiger Sanitäter. ${ }^{76}$ Wie bereits Kennedy so unterhielt auch Johnson regelmäßige

\footnotetext{
${ }^{73}$ Trotz der eingeleiteten Neuorientierung stand die fortgesetzte strategische Bedeutung Irans, allein schon aufgrund seiner geographischen Lage, weiterhin außer Frage. Der Joint Strategic Objective Plan für die erste Hälfte der 1970er Jahre erwähnte dann auch explizit die Gefahren einer neutralistischen Neuorientierung Irans und die möglicherweise daraus resultierenden ,serious security problems“. Vgl. JCS, Joint Strategic Objectives Plan for FY 1970-1974 (JSOP-70), o.D. [August 1964], FRUS 64-68, X, S. 112-41, hier 116.

${ }^{74}$ Vgl. Near East-South Asia IRG Meeting, 12.5.66, FRUS 64-68. XXII, S. 241-4.

${ }^{75}$ Tehran \#1397, 18.4.66, ebd., S. 232.

${ }^{76}$ Vgl. Bundy Memo, 4.12.65, ebd., S. 201; State \#683, 13.12.65, AID (IRAN) VIETS; die Botschaft sah die Entsendung der Einheit positiv: ,We beleive $\{$ sic $\}$ it excellent demonstration of Iranian support for US policy in Viet-Nam in face sheepishness of other Afro-Asian countries."; Iran hatte bereits im Vorjahr Südvietnam mit der Lieferung von Ölprodukten unterstützt. Vgl. State \#71, 23.8.65; Tehran \#2, 1.7.64, CF 64-66; Tehran \#790, 28.11.65; State \#718, 2.3.65, ,Iran, Vol. I, Cables, 1/64-12/65‘, NSF, Country File, Box 136, LBJL; das „Medical
} 
Konsultationen mit dem iranischen Monarchen über weltpolitische Fragen ab, was die übersteigerte Selbstwahrnehmung Mohammad Rezās als führender Staatsmann seiner Zeit zusätzlich stärkte. ${ }^{77}$ In der Summe etablierten sich in der ersten Hälfte der 1960er Jahre Grundzüge einer auf Dominanz ausgerichteten iranischen Regionalpolitik und Ambitionen auf einen zukünftigen Großmachtstatus, gestützt auf einer bereits hier wahrnehmbaren Hybris Mohammad Rezās. In der auf Südostasien fokussierten Johnson-Administration wurden diese Warnsignale aber ignoriert und der Nahostregion erst nach der Destabilisierung infolge des Juni-Krieges von 1967 wieder Aufmerksamkeit geschenkt.

VII. 2. Handlungsautonomie nach innen: Die Ablösung des Klientelstatus und die Durchsetzung monarchischer Dominanz

Ähnlich wie im außenpolitischen Sektor war seit Ende 1963 auch im Bereich der Innenpolitik eine im Vergleich zu den Tagen des reformistischen Interventionismus nahezu vollständige Abnahme amerikanischen Steuerungsvermögens bzw. Einflusses zu registrieren. Im Anschluss an die ,erfolgreichen“ Mağles-Wahlen vom September 1963 war Mohammad Rezā den zuvor eingeschlagenen Weg einer immer konsequenter durchgesetzten monarchischen Steuerung der Reformmaßnahmen sowie der Wirtschaftspolitik bei graduell zunehmender interner Repression weiter gegangen. Gerade die durch die rasant steigenden Öleinnahmen geschaffene relative Autonomie des Staates ermöglichte die Durchsetzung dieser neuen Interpretation der traditionellen Dominanz des politischen Systems in Iran durch den Hof. ${ }^{78}$

Im Mai 1964 waren die CIA und die anderen US-Nachrichtendienste in einer Einschätzung der Lage in Iran zu dem Schluss gekommen, die lange Zeit als unabänderlich angesehene revolutionäre Transformation Irans müsse nicht zwangsläufig zu einer umfassenden Umgestaltung der traditionellen sozialen und politischen Strukturen führen. Die jüngste Entwicklung in Iran erwecke eher den Eindruck, dass die Adaption auf Einflüsse von außen und den Modernisierungsdruck von innen auch ohne Anwendung revolutionärer Gewalt möglich sei, was letztendlich auf die Übernahme einer persönlichen Leitrolle Mohammad Rezās im Kontext einer „Revolution von Oben“ zurückzuführen sei. ${ }^{79}$ Mit der Weißen

Team" traf im Februar 1966 in der Provinz Kien Hoa in Südvietnam ein. Vergeblich hoffte man aber auf eine Ausweitung des iranischen Engagements, z.b. durch die Entsendung einiger Offiziere der amerikanisch ausgebildeten Gendarmerie. Vgl. Meyer Letter, 21.12.66, ,POL Iran Viet Nam (South) 1966', Lot 70 D 330 , NEA/IRN: 1964-66, Box 18.

${ }^{77}$ Vgl. Tehran \#778, 25.11.65, FRUS 64-68, XXII, S. 194.

${ }^{78}$ Zur steigenden Bedeutung des Hofs und dessen internen Prozessen vgl. AZIMI, Quest for Democracy S. 228-44.

79 Damit diese Annahme langfristige Gültigkeit beanspruchen könne, müsse allerdings in der Zukunft eine politische Unterstützungsbasis für die Reformvorhaben entstehen: „Even if he persists, the success of the Shah's initiative for evolutionary reform will depend in the long run on whether it evokes a response in the country sufficient to be translated into broadened political support for the regime.“; CIA, NIE 34-64 ,Iran“, 20.5.64, FRUS 64-68, XXII, S. 41-52, hier 49. 
Revolution habe ein Prozess umfassender Transformation eingesetzt, wenngleich das Tempo insbesondere der zentralen Landreform mit dem Abschluss der ersten Phase im September 1963 merklich verlangsamt worden sei. Vorerst aber stütze sich das Schah-Regime wie auch zuvor in erster Linie auf die Armee und die Sicherheitsorgane - einen neue politische Synthese, nach den frühen Vorstellungen auf der ,befreiten“ Bauernschaft aufbauend, zeichne sich noch nicht ab. Im Gegenteil, durch die Entfremdung der alten landbesitzenden Elite und des schiitischen Klerus habe die Opposition sogar neuen Zulauf erhalten. Wenn überhaupt, dann habe die bestehende Regierung neue Unterstützung seitens der sich mehr und mehr in den Bürokratien durchsetzenden neuen Manager- und Technokraten-Klasse erhalten, häufig im Ausland ausgebildet. Wenngleich nicht unkritisch gegenüber der Herrschaftsweise der Pahlawis eingestellt, sei diese aber dennoch vorwiegend pragmatisch auf die eigene Karriere ausgerichtet und mit begrenzten politischen Ambitionen ausgestattet. ${ }^{80}$ Diese neue Schicht dominiere auch den 21. Mağles, gewählt im September 1963. Insbesondere seien Regierungsangestellte und Beamte in Hasan 'Ali Mansurs „Partei des Neuen Iran“ (Hezb-e Irān-e nowin) zu finden, der inzwischen etwa 140 der 189 Abgeordneten zugewandt waren. ${ }^{81}$

Wie allgemein erwartet, löste Mansur den bisherigen Premier 'Alam im März 1964 ab. Der Ministerpräsident Mansur zeichnete sich in erster Linie durch unterwürfige Gesten und Reden gegenüber dem Monarchen aus, zeigte ansonsten aber nur sehr begrenzte Fähigkeiten, was Regierungsführung betraf. ${ }^{82}$ Die wirtschaftliche Situation hatte sich entgegen den Erwartungen im Laufe des Jahres 1964 nur unwesentlich gebessert - eine schlechte Ernte und Produktionsausfälle infolge der ersten Phase der Landreform machten Getreideimporte über das amerikanische PL-480-Programm in größerem Umfang als geplant notwendig. Trotz rasant steigender Öleinnahmen war die Mansur-Regierung im Dezember 1964 zu Steuererhöhungen gezwungen. Unter anderem wurde der Preis von Benzin auf einen Schlag verdoppelt, was insbesondere die urbanen Mittelschichten empfindlich traf. ${ }^{83}$ Neben der noch zu schildernden

\footnotetext{
${ }^{80}$ Vgl. ebd., S. 48.

${ }^{81}$ Offenkundig auf Ukas des Schahs gegründet und anfangs weitgehend ohne eigenes Programm oder irgendeine Basis in der Bevölkerung, ähnelte die Hezb-e Irān-e nowin europäischen Honoratiorenparteien des 19. Jahrhunderts. Außenpolitisch gab sich Mansur zu Beginn extrem pro-amerikanisch: ,the Party's identification with the United States may actually be excessive. A number of informants report that the Party's leadership, especially Hassan Ali Mansur, are freely boasting of their close contacts with the American embassy, and some comment has been heard that this is doing neither the new party nor the U.S. much good."; Tehran \#A-366, 4.1.64, POL 1 IRAN; von Beginn an gab es die weitverbreitete Meinung, Gespräche mit dem amerikanischen Professor Richard Pfaff der University of Colorado hätten den Schah von den Vorzügen eines Einparteiensystems zur Umsetzung seines Reformprogramms überzeugt. Die Partei dehnte sich im Laufe von 1964 langsam auf die wichtigsten Provinzzentren aus, wobei die ausgewählten Kandidaten zuvor genau vom SAWAK überprüft wurden. Vgl. Tehran \#A-346, 31.12.64, POL 12 IRAN, CF 64-66.

${ }^{82}$ Vgl. AzIMI, Quest for Democracy, S. $180 \mathrm{f}$.

${ }^{83}$ Vgl. Tehran \#A-254/\#A-296, 18.11./7.12.64, E 1 IRAN; die fortdauernde Krise hinterließ auch soziale Folgen. So berichtete die Botschaft z.B. besorgt über die stetig ansteigende Zahl Heroinabhängiger in Iran. Vgl. Tehran \#A-643, 19.5.64, SOC 11-5 IRAN, CF 64-66; der Kampf gegen den Drogenschmuggel und die Opiumproduktion war eine durchaus bedeutende Komponente des amerikanischen Nation Building in Iran. Auf US-Druck hin hatte der Schah 1955 den Opiumanbau in Iran verboten - die Produktion verlagerte sich bereits dann schrittweise nach Afghanistan. 1969 erlaubte Mohammad Rezā wieder den begrenzten Anbau innerhalb Irans, gegen amerikanischen Widerstand, und vorgeblich unter scharfer staatlicher Kontrolle. Vgl. Tehran \#A-182, 9.6.70; CIA Chief of the Free World Division Memo to CIA Heroin Coordinator, 7.7.71, FRUS 69-76, E-4, docs. 72, 136; CIA Intel Memo ER IM 72-90, 12.6.72, DNSA: IR00775; vgl. GINGERAS, Poppy Politics.
} 
Status Bill-Affäre waren es insbesondere diese wirtschaftlichen Einschnitte, welche die Mansur-Regierung innerhalb kurzer Zeit extrem unpopulär werden ließ. Die Ermordung Mansurs durch einen Attentäter im Januar 1965 wurde von Beobachtern vor allem auf diese Entscheidungen zurückgeführt. ${ }^{84}$

Ähnlich durchwachsen war zu diesem Zeitpunkt auch die Bilanz der Weißen Revolution zwei Jahre nach ihrer Propagierung durch den Schah. Die CIA analysierte im Dezember 1964 den Stand der Reformanstrengungen. Die Geschwindigkeit der weiterhin als zentralen Bestandteil der „Revolution von Oben“ betrachteten Landreform habe sich seit Einleitung der zweiten Phase im Oktober 1964 merklich verlangsamt. Dies sei zum einen vom Schah seit der Entlassung von Landwirtschaftsminister Arsanğāni im Frühling 1963 so gewünscht gewesen, zum anderen auf die administrativ weitaus größere Herausforderung der zweiten Phase zurückzuführen, die nun auf etwa die Hälfte der insgesamt etwa 50.000 Dörfer in Iran in Besitz kleinerer und häufig lokal anwesender Grundbesitzer Anwendung fand. Bislang hätten die Reformen viel Unruhe und ökonomische Unsicherheit erzeugt, mit ein Hauptgrund für die weiterhin stagnierende private Investitionstätigkeit. Von den anderen im Referendum von 1963 angekündigten Reformen war im Wesentlichen nur die Alphabetisierungskampagne vom Fleck gekommen. Angesichts der vielen Schwierigkeiten drohe eine neue Wirtschaftskrise und infolge dessen eine Vereitelung der langfristigen Pläne Mohammad Rezās: „At best, Iran appears headed for slower implementation of his program if no crisis diverts it entirely. "85 Die Botschaft in Teheran ging Mitte 1964 noch weiter, als sie behauptete, im Grund gäbe es überhaupt kein aktives Reformprogramm in Iran mehr, aber dennoch sei die große Mehrheit der Iraner von der Existenz eines solchen überzeugt. Dennoch fehle es seit der Verlangsamung des Programms an einer überzeugenden Ideologie, auf deren Grundlage echte politische Mobilisierung möglich sei. ${ }^{86}$ Der Versuch, mithilfe der neuen Einheitspartei ein Vehikel zur Massenmobilisierung zu schaffen, scheiterte vollständig, mangels der Unterstützung breiterer Volksschichten aber auch des Zögern des Monarchen, der fortwährend die Entstehung alternativer Machtzentren befürchtete und jede Entwicklung in diese Richtung, selbst wenn ursprünglich von ihm selbst angestoßen, sabotierte. Die meisten amerikanischen Beobachter stimmten dem Diktum des Zweiten Botschaftssekretärs Bill Miller zu, der die offizielle Staatspartei als „empty pseudo-party“ charakterisierte. ${ }^{87}$

\footnotetext{
${ }^{84}$ Vgl. AzIMI, Quest for Democracy, S. $183 \mathrm{f}$.

${ }^{85}$ CIA Special Report, ,Reform in Iran: Progress and Prospects ‘, 11.12.64, ,Iran, Memos [...]12/65 ', NSF, Country File, Box 136, LBJL; die Conclusion auch in FRUS 64-68, XXII, S. 116f.; vgl. auch BILL, Eagle and Lion, S. 164f.; Bill Miller bewertete später die Landreform als überstürzt und mittelfristig fatal: „Land reform as conceived in the 1960's was an inappropriate idea imposed by Western land reform theorists who tried to apply methods used elsewhere on a very complicated traditional land system."; William Green Miller OHI, 10.2.2003, FAOH; zur Mär vom herzlosen „Landlord“ vgl. auch William G. Miller OHI, 7.3.85, Transcript 2, IOHP, S. 15.

${ }^{86}$ Vgl. Tehran \#A-to State, 15.6.64, POL 1 IRAN, CF 64-66; ähnlich BILL, Eagle and Lion, S. 168.

${ }^{87}$ Miller Letter, 11.2.64, POL 7 IRAN, CF 64-66; trotz des Scheiterns des ohnehin nur halbherzig umgesetzten Einparteiensystems blieben die älteren Staatsparteien der späten 1950er Jahre formell existent, wenn auch politisch nun völlig bedeutungslos. Die „loyale“ Oppositionspartei Mardom hatte es 1966 gewagt, öffentlich kritische Fragen zum Haushalt zu stellen. Bezeichnend für die politische Situation war, dass die Partei angesichts einsetzender Kritik seitens der Regierung und des Schahs ernsthaft die Selbstauflösung erwog. Vgl. Tehran \#A164, 22.8.66, POL 2-3 IRAN, CF 64-66.
} 
Die Botschaft konstatierte, der revolutionäre Elan der frühen Jahre der Weißen Revolution sei rasch verflogen. Infolge des Fehlens einer integrativen Ideologie sei die Unterstützung für das Programm des Schahs nur oberflächlich, selbst in denjenigen Schichten, die am meisten vom System profitierten:

Since the opposition is weak, divided, and dispirited, the regime ought to be feeling happy and secure, particularly as it has important political assets in its favor. But one of the remarkable intangible factors in the present situation is that the regime has so few convinced supporters. Evidence of this is found at every turn: Prominent members of the New Iran Party who express the belief, quietly and privately, that their party is a sham and a fraud and that no political party can be expected to do useful work as long as the Shah's heavy hand rests on the decision-making process; hand-picked Majlis members who deplore 'American support' for a regime which they term a travesty of democracy; Civil Adjutants of the Shah, who belong to his most devoted supporters, yet who express the belief that Iran will never be able to solve its problems as long as there is no freedom of expression, no delegation of authority, and so little selection of personnel for merit; [...] These are not members of the opposition. They are members of the Establishment who, even while loyal to the Shah, are suffering from a profound malaise, from lack of conviction in what they are doing, from doubts whether the regime deserves to endure. ${ }^{88}$

Tatsächlich, so die Botschaft weiter, habe sich die Unterstützungsbasis der „unpopulären Diktatur" des Schahs infolge der Weißen Revolution weiter verkleinert. Die ,befreite Bauernschaft" existierte als solche nicht als politische Kraft und die Regierung schreckte angesichts des sozialrevolutionären Potentials auch vor einer umfassenden Mobilisierung zurück. Die große Mehrheit der Intellektuellen und der urbanen Mittelschichten seien trotz Reformprogramm der Führung weiter entfremdet, die früheren Systemstabilisatoren der landbesitzenden Schichten und des schiitischen Klerus nun verdeckt bis offen feindselig. Immer wichtiger, so die Botschaft in dieser - wenn angesichts ihres kritischen Grundtons für die Berichterstattung aus Teheran kaum repräsentativen - Analyse vom Juni 1964, seien die „extremely effective internal security services and the armed forces which receive special benefits and who constituted the true basis of power of the Shah. “89

Bob Komer im Weißen Haus unternahm im April 1965 einen erneuten Versuch, den Präsidenten angesichts der schleppenden Reformen in Iran für mögliche Gefahren zu sensibilisieren. Die Reform-Rhetorik Mohammad Rezās sei weitaus überzeugender als die bislang tatsächlich umgesetzten Maßnahmen. Angesichts rapide steigender Öleinnahmen sei der Schah , ,...] tempted to spend far too much on fancy military hardware and not enough on meeting his own people's rising expectations." ${ }^{\text {"90 }}$ Wie in den beiden vorangegangenen Unterkapiteln gezeigt, war man seitens der Administration aber nicht gewillt, das Mittel der Annual Review zugunsten einer gesünderen Balance zwischen Aufrüstung und Entwicklung

\footnotetext{
${ }^{88}$ Airgram \#A-702, 15.6.64, POL 1 IRAN, CF 64-66 \{Hervorheb. i. Orig.\}.

${ }^{89}$ Ebd. \{Hervorheb. v. Verf.\}; es überrascht daher nicht, dass gerade dieses Airgram, ein Ausnahmebeispiel kritischer Berichterstattung aus der Botschaft, nach der Revolution veröffentlicht wurde. Vgl. HERZ, A View from Tehran.

${ }^{90}$ Komer Memo, 15.4.65, FRUS 64-68, XXII, S. 139.
} 
einzusetzen. Entscheidend für diese Zurückhaltung war sicherlich das weiterhin präsente institutionelle Gedächtnis innerhalb der Administration, was neue Versuche reformistischer Einflussnahme in Iran kaum wünschenswert erscheinen ließ. Wirtschaftlicher Aufschwung überdeckte in seinen politischen Effekten bald immer stärker die Reformbemühungen im Zuge der Weißen Revolution. Anfang 1966 konstatierte die CIA, die Landreform setze sich nur schleppend fort. Die ehemaligen Landbesitzer hätten sich zudem inzwischen auf die Umverteilung eingestellt und ihre wirtschaftliche Vormachtstellung - wenn auch nicht ihren herausgehobenen politischen Status - anderweitig gesichert. Zwar sei grundlegender sozialer Wandel infolge der Reformen unumgänglich, doch glaubte man nicht mehr an eine revolutionäre Transformation infolge der Landreform. Dennoch habe gerade die Landverteilung an die zuvor abhängigen Bauern das Prestige des Schahs auf dem Land enorm gesteigert und insgesamt zur Stabilisierung des Landes beigetragen. Die einzig nennenswerte weitere Reform sei das Alphabetisierungskorps, das offenbar mit großem Enthusiasmus umgesetzt werde. ${ }^{91}$

Mitte der 1960er Jahre hellte sich allerdings das in Washington registrierte Bild der inneren Lage in Iran merklich auf. Die Steigerung der Öleinnahmen, eine Serie von guten Ernten, das Ausbleiben negativer Wirkungen der Landreform auf die landwirtschaftliche Produktion und ein besseres Investitionsklima machten sich nunmehr bemerkbar. Die neue Regierung unter Howejdā hob die Austeritätsmaßnahmen Mansurs auf und orientierte sich in ihrer Wirtschaftspolitik nun stark an den Interessen der neuen Wirtschaftselite, die traditionelle Händlerschicht des konservativ-religiösen Basars bewusst benachteiligend. ${ }^{92}$ Mitte 1966 meldete die optimistischer werdende Botschaft in Teheran, dass die immer sichtbarer werdenden Auswirkungen eines Wirtschaftsbooms im ganzen Land $\mathrm{zu}$ einer spürbaren Abnahme des politischen Aktivismus geführt hätten: „For years the political atmosphere has not been as sluggish, as self-satisfied, or as resigned to the status quo."93 Die urbanen Mittelschichten seien nun tatsächlich im Begriff, sich von Hauptträgern des politischen

\footnotetext{
91 Vgl. CIA, NIE 34-66, ,Iran', 24.3.66, ,34, Iran', NSF, National Intelligence Estimates, Box 6, LBJL; Conclusions auch in FRUS 64-68, XXII, S. 223f.; eine umfassende Studie der Botschaft im Sommer 1964 hatte ergeben, dass etwa $40 \%$ der zu verteilenden Dörfer in der ersten Phase der Landreform letztlich nicht betroffen waren, da die großen Landbesitzer entweder den Besitz rechtzeitig unter Familienmitgliedern aufgeteilt hatten, häufig erst nach dem Stichtag mithilfe korrupter Provinzverwalter. Man schlussfolgerte, dass ,,the great majority of the major landlords in Iran have not been seriously hurt by land reform.“; Tehran \#A-726, 25.6.64, POL 6 IRAN; die Botschaft wies Mitte 1964 darauf hin, es habe im Grunde kaum internen Druck zugunsten der Durchführung einer Landreform gegeben - zudem sei die Bauernschaft nie ein politisches Problem gewesen. Vgl. Tehran \#A-702, 15.6.64, POL 1 IRAN, CF 64-66; Tehran \#A-926, 30.11.68, E 12 IRAN, CF 67-69; das Literacy Corps hatte bis Mai 1967 bereits 26.000 Wehrpflichtige aufs Land als temporäre Lehrkräfte entsandt - der Anteil der Landbevölkerung mit wenigstens rudimentärer Bildung war infolgedessen von etwa $8 \%$ auf $20 \%$ gestiegen. Andere Reformmaßnahmen wie die Nationalisierung der Wälder, Privatisierung von Staatsbetrieben, Profitbeteiligungen von Arbeitern waren entweder unspektakulär bzw. wurden nur halbherzig implementiert. Die ursprünglichen sechs Programmpunkte der Weißen Revolution wurden schrittweise bis Ende der 1960er Jahre auf zwölf erweitert, u.a. mit der Einführung eines Health Corps und eines Development Corps. Vgl. Tehran \#A-630, 22.5.67, E 5 IRAN, CF 67-69; zum Literacy Corps vgl. SABAHI, Literacy Corps.

92 Tehran \#A-105, 17.8.65, POL 2 IRAN, CF 64-66; die rasche wirtschaftliche Verbesserung beschleunigte laut der Botschaft den amerikanischen Einflussverlust: ,As in many other parts of the world, American influence is no longer a concomitant of economic assistance and its nature is changing as the financial strength of our partners permits them to act with greater self-assurance."

93 Tehran \#A-164, 22.8.66, POL 2-3 IRAN, CF 64-66.
} 
Widerstands in Unterstützer des Status Quo zu wandeln, die neue Atmosphäre sei von einem Hauch von „embourgoisement“ durchdrungen. ${ }^{94}$ Tatsächlich gab es sogar eine Reihe prominenter „Überläufer“ von der Opposition zum Regime, überzeugt in Teilen durch die nun in ihrer Wirkung kaum noch $\mathrm{zu}$ bestreitenden sozialtransformativen Wirkungen der „Revolution von Oben“, aber auch häufig durch die sich nun ergebenden Karrierechancen für die gebildete Elite. Später in Iran als der Prozess der „Bestechung der Intellektuellen“ (rešwat dādan be-roušan-fekrān) diffamiert, war eine reale Unterstützung für das Regime aber nur in den seltensten Fällen zu beobachten. ${ }^{95}$ Die Wahrnehmung opportuner Karrieremöglichkeiten bei gleichzeitigem fortgesetzten ,,inneren Widerstand“" war aber eher der Regelfall.

Trotz des wahrnehmbaren Verlusts der Unterstützung der traditionellen Oberschicht und der großen Landbesitzer für das Regime infolge der Weißen Revolution und der minimalen politischen Unterstützungsbasis in der Gesellschaft war die Machtposition Mohammad Rezās ab Mitte der 1960er Jahre weitaus gefestigter als in den vorangegangenen Jahren. Rasant steigende finanzielle Ressourcen durch die Öleinnahmen und die Ablösung der bisherigen amerikanischen Vormundschaft hatten die Handlungsoptionen des Schahs erheblich erweitert. Die zentralen Staatsorgane büßten immer mehr an Autonomie ein und wurden nun reine Ausführungsorgane des Willens Mohammad Rezās. Die fortgesetzte Zusammenarbeit der USA mit den repressiven Organen hatte deren Effizienz zudem merklich gesteigert. SAWAK und Polizei waren nun noch wichtiger als zuvor für die Stabilität des Pahlawi-Regimes. Die einzige Institution in Iran, die theoretisch gegen das Gewaltmonopol dieser Kräfte ein Gegengewicht hätte bilden können, die Streitkräfte, zeichnete sich durch größte Servilität gegenüber dem Schah aus. ${ }^{96}$ Eine Studie der Botschaft vom Sommer 1966 über die 31 höchsten Offiziere im Land stellte fest, dass alle ,[...] owe their attainment of high rank more to loyalty than to military capacity, and none of them can be described as being inclined to independent action. “97 Zudem verhinderte Mohammad Rezā durch persönliches Intervenieren und häufige Umbesetzungen in den obersten Rängen die Entstehung einer möglichen Machtkonzentration in den Händen fähiger Offiziere bereits im Ansatz. Finanzielle Großzügigkeit gegenüber dem Militär und Erfüllung fast jeden Beschaffungswunsches taten ein Übriges, um Loyalität wie politische Apathie des Militärs sicherzustellen. Einer der höchsten Generäle stellte nach der Revolution fest, das Militär sei im Grunde die private Armee Mohammad Rezās und nicht die

\footnotetext{
${ }^{94}$ Ebd.: die Botschaft registrierte, die Schicht der reichsten Iraner werde inzwischen von Personen dominiert, die den urbanen Mittelschichten oder sogar kleinbürgerlichen Verhältnissen entstammten. Auffallend sei die häufige Herkunft aus den religiösen Minderheiten. Für zeitgenössische akademische Einschätzungen vgl. ZoNIS, Political Elite; BILL, Politics of Iran; vgl. auch BASHIRIYEH, State and Revolution, S. $39 \mathrm{f}$.

${ }_{95}$ Vgl. BILL, Eagle and Lion, S. 164f., 167f.; vgl. VAKILI-ZAD, Modernization and Development; MATIN-ASGARI, Intellectual Accommodation.

${ }_{96}$ AzIMI, Quest for Democracy, S. 251, spricht von einer Kultur der Fügsamkeit in den Streitkräften.

${ }^{97}$ Tehran Airgram \#A-858 to State, 28.6.66, POL 6 IRAN, CF 64-66, RG 59, NACP; nur vieren der 31 traute die Botschaft politisch exponiertes Handeln in bestimmten Umständen, gemeint war die Beseitigung des Schahs, zu. Die vier waren die Generäle Ferejdun Ğam, Bahrām Ārjānā, Mohammad Xātami und Fathallāh Minbāšijān. Die Wertung von Azimi, die USA hätten „,considerable control“ über die Streitkräfte ausgeübt, ist sicherlich unzutreffend. Siehe AZIMI, Quest for Democracy, S. 250; vgl. auch Tehran \#A-657, 3.6.67, POL 6 IRAN, CF 6769.
} 
Armee des Landes bzw. der Nation gewesen. ${ }^{98}$

Angesichts der politischen Bedeutungslosigkeit der Streitkräfte und der Entmachtung einstmals autonomer Machtzentren wie dem Mağles und der Bürokratie verstärkte sich der bereits seit 1949 abzeichnende weitere Einflussgewinn des Hofs als direktem Ausführungsarm der unangefochtenen Dominanz des Schahs. Zugleich zeigte sich auf amerikanischer Seite ein gestiegenes Interesse am direkten Umfeld des Schahs und seiner Familie, im Gegensatz zur früheren Konzentration auf urbane Mittelschichten oder „klassische“ Einflussträger wie Offizierskorps und Sicherheitsorgane. Geringer Einfluss auf Mohammad Rezā wurde seiner direkten Verwandtschaft zugeschrieben. Die Familie sei angesichts ihrer Involvierung in einer ganzen Reihe von legalen wie zweifelhaften Wirtschaftsprojekten eher eine Belastung denn eine Stärkung der persönlichen Herrschaft des Schahs. Einzig allein seiner Zwillingsschwester Ašraf - ,[...] has a near legendary reputation for financial corruption and for successfully pursuing young men“, so eine CIA-Studie später - und seiner Mutter Tāğ-o-1-Moluk wurden nachhaltiger Einfluss auf seine Entscheidungen zugeschrieben. ${ }^{99}$ In jüngerer Zeit sei zudem der Einfluss seiner dritten Frau Farah, geborene Farah Dibā, gestiegen, die über breite Popularität in der Bevölkerung verfüge. Unter den Höflingen dominiere die Mediokrität - nur wenige hätten ausreichend Wissen und Vertrauen des Herrschers, um eine Rolle bei der Entscheidungsfindung zu spielen. Der Hof selbst sein ein ,hotbed of byzantine scheming.“100 Asadollah 'Alam, der Hofminister und Jugendfreund Mohammad Rezās, zudem ehemals selbst Ministerpräsident, galt als der engste Vertraute. Allerdings zeigen seine posthum veröffentlichten Tagebücher, dass selbst er in viele Bereiche der Regierungsführung keine Einsicht hatte. ${ }^{101}$ Fakhreddin Azimi zufolge vereinte 'Alam ,[...] the roles of a reliable friend, an obedient servant, an exalted pimp, an unctuous jester, and an efficient functionary who did his utmost to cater for all royal needs and fancies. " ${ }^{102}$ Er sei letztlich der zweitwichtigste Mann Irans gewesen.

Andere Höflinge und Offizielle von Bedeutung, die dem inneren Kreis rund um den Herrscher zugerechnet wurden, waren der Quasi-Privatsekretär und Leiter des "Special Office“, Nosratallah Mo inejān, der Leiter des Special Intelligence Bureau innerhalb SAWAKs und anschließend des Imperial Inspectorate und Jugendfreund Hosejn Fardust, Leibarzt und

\footnotetext{
${ }^{98}$ So General Ferejdun Ğam, zitiert aus AzIMI, Quest for Democracy, S. 251; vgl. Tehran \#A-680, 6.5.76, Asnād 7, S. 205-18; KAZEMI, Military and Politics.

${ }_{99}$ Vgl. CIA Study, ,Elites and the Distribution of Power in Iran ' [im Folgenden: CIA Elites Study][in Teilen auch in FRUS 69-76, XXVII, S. 482-8], Februar 1976, Asnād 7, S. 1-83, hier 27; noch schärfer auf S. 10: ,a lady possessed of a greedy nature and nymphomaniac tendencies."; Ašrafs Engagement für Frauenrechte sei eine Idee Mohammad Rezās gewesen, um ihr extrem negatives Image in Iran aufzubessern, aber auch um ihre Energie etwas von ihren ansonsten „shady activities“ abzulenken, so die Studie; vgl. Tehran \#A-19, 28.1.75, ebd., S. 130-44.

${ }^{100}$ CIA Elites Study, S. 10; diese Einschätzung wird mehr als bestätigt durch den Bruder des Premiers. Vgl. HOVEYDA, Fall of the Shah; dieser bestätigt auch den sich aus vielen Quellen ergebenden, angesichts der Geschichte iranischer Zivilisation etwas schockierenden Eindruck einer geistig außergewöhnlich anspruchslosen Hofgesellschaft. Dominierend waren eher die sportlichen Aktivitäten Mohammad Rezās.

${ }^{101}$ Vgl. CIA Elites Study, S. 30f.; vgl. AlAM, Shah and I, S. 22-5.

${ }^{102}$ AZIMI, Quest for Democracy, S. 242.
} 
ebenfalls Jugendfreund Abdol Karim Ajādi, die früheren Premierminister Manučehr Eg̉bāl und Ğa far Šarif-Emāmi, der neue Ministerpräsident Amir 'Abbās Howejdā, der Imām-e Ğom eh von Teheran, Hasan Emāmi, Außenminister Ardešir Zāhedi sowie Luftwaffenchef Mohammad Xātami. ${ }^{103}$ Diese Personen seien zugleich Kanäle für die Informationsvermittlung an den Schah und ausführende Organe für einmal getroffene Entscheidungen: „He seeks information. He does not seek advice, and few in Iran would dare to give him any. He decides, others execute. "104 Personen seien daher wichtiger für die Entscheidungsformulierung in Iran als Institutionen, wobei der Schah bewusst eine „Teile und Herrsche“-Politik gegenüber diesen Personen verfolge und Rivalitäten aktiv fördere, um seine Stellung als alleinige und unangefochtene Entscheidungsinstanz - als eine Art Appellationsgericht - zu zementieren. Die stark zentralisierte und hoch personalisierte Entscheidungsstruktur fördere rasche Entscheidungen, führe aber zugleich zum Ausschluss von Fachkompetenz, dem Vermeiden jeglicher Eigeninitiative und fördere zudem die bewusste Desinformation Mohammad Rezās, um Entscheidungen zu manipulieren bzw. negative Konsequenzen einmal getroffener Entscheidungen zu vertuschen. ${ }^{105}$

Die eigentliche Regierung war nunmehr de facto nur noch Befehlsempfänger des Herrschers. ${ }^{106}$ Der bisherige Finanzminister und neue Ministerpräsident Amir 'Abbās Howejdā galt zwar als exzellenter Administrator - er hatte insbesondere in der NIOC in führender Position erfolgreich gewirkt - und gewann zeitweise eine moderate Popularität innerhalb Irans aufgrund der ihm zugeschriebenen wirtschaftlichen Verbesserungen und einer auch von Gegnern attestierten, für

\footnotetext{
${ }^{103}$ Vgl. CIA Elites Study, S. 31-37; Fardust sollte in der Revolution den Schah verraten. Als einer der wenigen hohen Offiziellen überstand er die Revolutionswirren unbeschadet und half offenbar auch beim Aufbau einer Nachfolgeorganisation des SAWAK für die Islamische Republik. Zu seiner Tätigkeit im SAWAK und im Inspektorat vgl. auch FARDUST, Rise and Fall, S. 207-71; Tehran \#A-85, 12.5.73, POL 6 IRAN, CF 70-73; zu Fardusts zweifelhaften Erinnerungen vgl. auch ABRAHAMIAN, Tortured Confessions, S. 159-62; vgl. MiLANI, Eminent Persians: I, S. 438-44; Ajādi wurde in der CIA-Studie u.a. als „,major channel through which the Shah dabbles in commercial affairs" bezeichnet. Xātami galt lange als starker Mann im Falle eines Ablebens des Schahs. Er kam bei einem Segelflugzeug-Unfall 1975 ums Leben. Eine eingehende Untersuchung des engsten Kreises rund um Mohammad Rezā bei ZONIS, Majestic Failure, S. 115-49; Zāhedi wurde von amerikanischer Seite als Luftikus und intellektuelles Leichtgewicht (,,a Mixed-Up Kid“) wahrgenommen. Vgl. Bowling Memo, 19.1.62, ,Iran, $1961[\ldots]^{*}$, NSF, RWK, Box 424, JFKL; MemCon, 3.8.73, FRUS 69-76, XXVII, S. 129-33; umso erstaunlicher ist die einflussreiche Rolle Zāhedis später in der Revolutionszeit, gerade bei Sicherheitsberater Brzezinski. George Ball beschrieb seine Rolle als ,in effect dictating American policy [...]“; Eintrag vom 17.1.79 in SCHLESINGER, Journals, S. 397; vgl. auch Precht Letter, 19.12.78, Asnäd 13, 5, S. 16.

${ }_{104}$ CIA Elites Study, S. 10; zum Herrschaftsstil des Schahs vgl. auch GraHam, Illusion of Power, S. 132-53.

${ }^{105}$ Vgl. Tehran \#A-132, 22.7.76, Asnād 7, S. 102-27; dieses Airgram nannte zusätzliche Personen von Bedeutung: SAWAK-Chef Ne' mat-ollāh Nassiri, Armeechef General Ǵlām- 'Ali Owejsi, der Vertraute für Rüstungseinkäufe, General Hasan Toufānijān (,that Iranian military Rasputin named Toufanian“), Stabschef Golām-Rezā Azhari, Finanzminister Ğamšid Āmuzegār sowie Wirtschafsminister Hušang Ansāri; , Rasputin“ in Meyer Letter, 20.8.66, DEF 12-5 IRAN, CF 64-66; zu Toufānijān vgl. MILANI, Eminent Persians, Bd. 1, S. 490-4; , The Pahlavi monarchy failed to develop a solid basis of power [...]", konstatierte der Historiker Fakhreddin Azimi in seiner Überblicksgeschichte des Autoritarismus im Iran des 20. Jahrhunderts. AzIMI, Quest for Democracy, S. 224; Azimi konstatiert das Fehlen einer loyalen Aristokratie bzw. eines Funktionsadels, gesteht aber zugleich ein, dass es der Monarchie zeitweilig durch Steuerung und Allokation der staatlichen Mittel gelang, eine politische Klientel zu generieren und zu unterhalten. Ebd.; FATEMI, Shah's Modus Operandi, kommt zu ähnlichen Schlüssen. Der Schah habe spätestens ab Ende der 1960er Jahre eine Entscheidungsorganisation errichtet, die einzig und allein darauf gerichtet war ,\{to make; R.P.\} himself the sole decision-making authority in every significant phase of Iran's political affairs."; ebd., S. 49; für alternative Sichtweisen vgl. AFKHAMI, Pahlavi Monarchy; und SCHAYEGH, Governmental Constraints; während der Revolution erkannte man diese Herrschaftsmethode Mohammad Rezās als eine der zentralen Ursachen für den Umbruch. Vgl. Washburn Letter to ,George‘, 18.9.78, Asnād 12, 3, S. 5863.

${ }^{106}$ Vgl. Tehran \#A-132, vorherig. Anm., S. 109.
} 
iranische Politiker durchaus unüblichen Volksnähe und persönlichen Bescheidenheit. ${ }^{107}$ Andererseits bestand während seiner langjährigen Ministerpräsidentenschaft bis August 1977 nie ein Zweifel, dass er letzten Endes nur Ausführungsorgan Mohammad Rezās war, der die Ausrichtung iranischer Politik nach innen wie außen nunmehr fast komplett im Alleingang bestimmte. ${ }^{108}$ Die praktische Aufhebung der verbliebenen politischen Autonomie des Amtes des Premiers und die Entscheidung Mohammad Rezās, selbst öffentlich als Initiator bedeutender politischer Maßnahmen aufzutreten, ließen dennoch einen, wenn auch begrenzten Handlungsspielraum. Belastend für Howejdā waren aber nicht nur seine Servilität gegenüber dem Monarchen, sondern auch die von seinen politischen Konkurrenten immer wieder verbreiteten Diffamierungen, vor allem die Behauptung, er sei ein Angehöriger der religiösen Minderheit der Bahai, bei religiösen Schiiten verhasst als Häretiker und fortwährend stilisiert als fünfte Kolonne ausländischer Mächte. ${ }^{109}$

Die Botschaft führte die allgemeine Stabilisierung der iranischen Innenpolitik zwar zuvorderst auf die - vorwiegend im ökonomischen Bereich zu findenden - Erfolge der Zentralregierung zurück, der es auf diese Weise gelungen sei, wenigstens in begrenzter Form Unterstützung der wichtigsten Gesellschaftsteile zu erlangen. Ohne Frage spielte aber die zunehmende Repression der staatlichen Organe eine gleichsam bedeutende, wenn nicht wichtigere Rolle. Zwischen 1963 und 1966 nahm die Unterdrückung in augenfälliger Weise zu. ${ }^{110}$ Im Mai 1964 berichtete die Teheraner Botschaft, dass ,[...] the Shah's control of his country is, if anything, still tighter than it was two years ago." "111 NEA meldete bereits im Frühjahr 1964 an die Special Group (CI), dass sich in den vergangenen Monaten die Bereitschaft „unzufriedener Gruppen“ - gemeint waren die nationalistische Opposition in den urbanen Mittelschichten, tribale Elemente im Süden und der regimekritisch eingestellte Teil der schiitischen Geistlichkeit - „to engage in anti-regime adventures" weiter vermindert habe. ${ }^{112}$ In den folgenden Jahren blieb diese Einschätzung weitgehend unverändert. Der ,gesteuerte Pluralismus“ der Vorjahre, das

\footnotetext{
107 Ausgewogen die Bewertung Howejdās in AzIMI, Quest for Democracy, S. 186f.; eher apologetisch die Darstellung in Milani, The Persian Sphinx; sehr kritisch zu Milanis Darstellung ist MATIN-AsGARI, Transparent Sphinx; vgl. auch CHEHABI, Hoveyda.

${ }^{108}$ Noch im Frühling 1965 hatten gut informierte iranische Beobachter damit gerechnet, die Regierung Howejdā dürfte nicht viel länger als ein bis zwei Monate überdauern. Vgl MemCon, 11.4.65, ,POL 1 Gen. Policy. Background. 1965', Lot 69 D 490, NEA/IRN: 1964-66, Box 12; Tehran \#A-559, 12.2.66, POL 15-1 IRAN, CF 64-66.

${ }^{109}$ Laut Milani, The Persian Sphinx, S. 43f., 47, 316, stammte er aus einer Familie mit Verbindungen zu den Bahai, war aber selbst kein Mitglied der Gemeinschaft. AZIMI, Quest for Democracy, S. 185, bezeichnet ihn als Sohn eines bekannten Diplomaten und Bahai-Mitglieds, wobei er selbst vollkommen säkular eingestellt gewesen sei. Howejdā wurde zudem immer wieder als Freimaurer und aufgrund angeblicher homosexueller Neigungen diffamiert. Die Mitgliedschaft in einer Loge war allerdings im Iran dieser Zeit durchaus Konvention. Vgl. MILANI, Eminent Persians: I, S. 193-204; der Schah-Vertraute Ga far Šrif-Emāmi berichtete später, er habe Anfang der 1960er Jahre im Auftrag des Schahs 18 existierende Freimaurerlogen zusammengefasst zur Iran-Loge unter ihm selbst als Großmeister, um die eigenen Netzwerke zu stärken und die staatliche Kontrolle dieser Aktivitäten zu verstärken. Der Schah selbst war offenbar trotz vieler anderslautender Gerüchte selbst kein Mitglied einer Loge. Vgl. LADJEVARDI, Xāterāt-e Šarif-Emāmi, S. 233-43.

110 Ähnlich die Einschätzung der Briten, die allerdings die von ihnen konstatierte Ineffizienz der Sicherheitsorgane als zentrale Schwäche des Regimes ausmachten. Vgl. British Embassy Letter, 7.9.66, ,POL 12 [...]1966', Lot 70 D 330, NEA/IRN: 1964-66, Box 18.

${ }^{111}$ Tehran \#1090, 28.5.64, FRUS 64-68, XXII, S. 58.

112 Jernegan Memo, 2.3.64, FRUS 64-68, XXII, S. 14.
} 
(zumindest begrenzte) Tolerieren oppositioneller Meinungen und (in Teilen) auch deren Organisation, die Hinnahme öffentlicher Kritik an der Politik der jeweiligen Regierung, das Zulassen eines relativ weiten Pressespektrums, um abweichende Positionen zu ventilieren und zu kanalisieren, all das wich nun einer zunehmend bleiernen und sich stetig verschärfenden Kontrolle von oben. Nach den Juni-Unruhen von 1963 hatte die Obrigkeit noch einigermaßen zurückhaltend reagiert. Von fünf Todesstrafen gegen Aufwiegler in Teheran wurden zwei vollstreckt. Gegen die schiitische Geistlichkeit selbst aber hielt man sich ansonsten - wohl auch aus Furcht vor weiterer Eskalation - merklich zurück. Dagegen ging man z.B. gegen die Führer der Stammesaufstände von 1963 in der Provinz Fārs rücksichtslos vor - 1964/65 wurden elf Aufständische hingerichtet. ${ }^{113}$ Amerikanische Beobachter waren zudem konsterniert über die bevorzugte Methode des Schahs für die Bekämpfung der aufständischen Stämme: NapalmAttacken auf die Zelte der Nomaden. ${ }^{114}$ In den Gebieten der kurdischen und arabischen Minderheiten wich die bisherige Politik der Duldung begrenzter, vorwiegend kulturell ausgerichteter Aktivitäten schrittweise ebenfalls einer Politik der harten Hand. ${ }^{115}$

Auch gegenüber der politischen Opposition machte sich die härtere Gangart nun bemerkbar. Zwar hatte man die Führungsfiguren der Nationalen Front nur wenige Monate nach den Unruhen des Sommers 1963 wieder auf freien Fuß gesetzt. Allerdings verblieben die nichtsäkularen Teile der nationalistischen Opposition, Mehdi Bāzargāns „Freiheitsbewegung Irans“ (Nehzat-e Āzādi-je Irān), in Haft. Bāzargān, im Jahr 1979 nach der Revolution für einige Monate iranischer Ministerpräsident, und einige seiner Mitstreiter wurden von einem Militärgericht abgeurteilt und zu langjährigen Haftstrafen verurteilt. ${ }^{116}$ Khomeini selbst wurde im April 1964 auf freien Fuß gesetzt. ${ }^{117}$ Er hatte zuvor angeblich eine Vereinbarung mit der Mansur-Regierung getroffen, sich in der Zukunft politischer Aktivitäten zu enthalten. Allerdings machte Khomeini nach seiner Freilassung keine Anstalten, sich an diese Absprache zu halten. Noch im April griff er in einer Rede die Regierung scharf an und kritisierte die hohen Militärausgaben, die Beziehungen zu Israel, den ausländischen Einfluss und die Verletzung der

\footnotetext{
113 Vgl. Tehran \#282, 19.11.66, POL 29 IRAN, CF 64-66; die drei seit 1954 im Exil lebenden Ġašḡà i-Brüder hatten bereits im November 1963 Verhandlungen mit der Regierung über eine Rückkehr nach Iran aufgenommen. Vgl. Tehran \#A-301, 30.11.63, POL 13-10 IRAN, CF 63.

${ }_{114}$ Dies war offenbar eine Reaktion auf den Verlust von zwei Kompanien der Spezialkräfte der Luftlandetruppen in einem Hinterhalt der Stämme. Vgl. After Action Report, encl. to Moses Memo, 19.3.64, ,POL 23 Internal $[\ldots ..] 1964^{\circ}$, Lot 69 D 178, NEA/IRN: 1964-66, Box 4.

15 Allerdings wich zugleich die vorherige ökonomische Vernachlässigung der Minderheitengebiete einer neuen Aufmerksamkeit, mit Zielrichtung auf langfristige Assimilation. Vgl. Tabriz \#A-9, 18.9.65, POL 13-3 IRAN; Khorramshahr \#A-57, 17.6.64; Tabriz \#A-35, 22.4.64, POL 29 IRAN; Tabriz \#A-17, 7.1.65, SOC 2-1 IRAN, CF 64-66; Jernegan Memo, 2.3.64, FRUS 64-68, XXII, S. 13-18, hier 14.

${ }^{116}$ Bāzārgan und der andere Mitbegründer der ,Freiheitsbewegung“, $\bar{A}$ jatollāh Mahmud Tālegāni, wurden zu zehn Jahren Einzelhaft verurteilt. Letzterer verbrachte die Jahre bis zur Revolution vorwiegend im Gefängnis, wohingegen Bāzargān bereits 1967 vom Schah begnadigt wurde und sich danach vorerst ins Privatleben zurückzog und nicht mehr politisch aktiv war. Ein Jahr nach dem Prozess wurden zudem die Anwälte der Oppositionellen in einem weiteren Schauprozess zu Haftstrafen verurteilt, offenbar als Abschreckung intendiert. Vgl. CHEHABI, Liberation Movement, S. 179-84; SiAvOSHI, Liberal Nationalism, S. 94.

${ }^{117}$ Bezeichnend für den stark gesteigerten Bekanntheitsgrad des Geistlichen war die Tatsache, dass die Regierung ein Panzerbataillon nahe Gom stationierte, um im Falle eventuell ausbrechender Unruhen gewappnet zu sein. Vgl. Tehran \#882, 6.4.64, FRUS 64-68, XXII, S. 26.
} 
Verfassung durch die autokratische Herrschaft des Schahs. ${ }^{118}$

Inzwischen bewerteten manche amerikanische Botschafter die religiöse Opposition als gefährlicher als die ,alte“ nationalistische Opposition. Khomeini sei nun der populärste Mann in ganz Iran, so lautete bereits die Warnung eines Mitglieds des verbliebenen proamerikanischen Netzwerks Anfang 1965. ${ }^{119}$ Inzwischen galt er zudem als respektabler Kandidat für die Marğa'ijja. ${ }^{120}$ Die Botschaft berichtete Mitte 1965 über mehrere Zwischenfälle in Ġom. Es gebe ,[...] disquieting evidence of religious anti-government propaganda that includes nationalistic, anti-American themes in terms that almost smack of communist inspiration", ein Hinweis auf die Irritationen, die diese neue Form von fundamentalistisch-religiös inspiriertem Widerstand in der Botschaft hinterließ. Tatsächlich war man über die religiösen Gruppen kaum oder nur unzureichend informiert. Die Kontakte der Botschaft ins religiöse Milieu umfassten nur etwa ein halbes Dutzend Personen, größtenteils zudem der Regierung nahestehend und damit kaum ergiebig, was die oppositionellen Gruppen anging. Ein Offizieller beklagte sich über die Schwierigkeiten für einen Ausländer, und gerade für einen Christen, sich mit dem schiitischen Milieu in Iran vertraut zu machen. Zudem sei der Anti-Amerikanismus gerade bei den religiösen Oppositionellen besonders stark ausgeprägt so hatte sich z.B. der Khomeini nahestehende Ājatollāh Ġomi einem Gespräch verweigert. ${ }^{121}$ NEA wandte sich schließlich gemeinsam mit INR im State Department an die CIA in der Hoffnung, mehr über die „obscurantist religious opposition groups in Iran“ zu erfahren. ${ }^{122}$ Offenbar schenkten die Sowjets dem Phänomen größere Aufmerksamkeit und steigerten ihre Aktivitäten im religiösen Milieu Irans. ${ }^{123}$

Die stark geschwächte Tudeh-Partei, zudem intern zerrissen durch ideologische Flügelkämpfe nach der Entstehung eines maoistischen Flügels, wurde ab Mitte der 1960er Jahre erneut zur Zielscheibe staatlicher Verfolgung, offenbar in Teilen als „Ausgleich“ für die diplomatische

\footnotetext{
118 Vgl. Tehran \#890, 8.4.64, ebd., S. 26f.; offenbar bemühte man sich auch seitens der Regierung, Khomeinis Aufstieg und mögliche Inanspruchnahme der Marğa'ijja durch Einflussnahme auf loyale religiöse Kreise einzudämmen. Mansur und SAWAK-Direktor Pākrawān hatten den Schah von den Vorteilen einer Freilassung Khomeinis noch vor dem Moharram-Passionsfeiern überzeugt. Zur Aprilrede Khomeinis vgl. Tehran \#967, 25.4.64, ebd., S. $33 \mathrm{f}$.

${ }_{119}$ Vgl. MemCon, 27.1.65, ,POL 1 Gen. Policy. Background. 1965‘, Lot 69 D 490, NEA/IRN: 1964-66, Box 12; offenbar versuchte der KGB, die anti-amerikanischen Einstellungen der religiösen Opposition mittels Propaganda und Dokumentenfälschung noch zu verstärken. Vgl. Fake Rockwell Letter to ,James', 6.11.64, encl. to Tehran \#A426, 11.2.65, POL 30-2 IRAN, CF 64-66.

${ }^{120}$ Bill Miller sprach unter anderem mit dem Teheraner Universitätsprofessor und Philosophen Sejjed Hosejn Nasr, früher den Reformisten des pro-amerikanischen Netzwerks zugerechnet, der sich nach seiner Rückkehr von einem mehrjährigen USA-Studienaufenthalt aber der Religion zugewandt hatte und aufgrund seines Interesse an der schiitischen Glaubenslehre und der eigenen Frömmigkeit enge Kontakte zu den oppositionellen religiösen Kräften und zu Ājatollāh Khomeini selbst hatte. Vgl. MemCon, 8.5.64, ,POL 13-6 Religious [...]', Lot 69 D 178, NEA/IRN: 1964-66, Box 4.

${ }^{121}$ Selbst das kurze Gespräch mit dem Geistlichen war offenbar vom SAWAK aufgezeichnet worden. Vgl. Herz Letter, 21.2.66; allgemeine Informationssammlung über die Geistlichkeit gehörte allerdings zum Tagesgeschäft. So erstellte die Botschaft im Juni 1964 z.B. eine umfassende Analyse des schiitischen Klerus und der Geschichte seiner Entfremdung vom Regime. Als Fazit stand aber auch hier der zu erwartende Niedergang des geistlichen Einflusses auf die Bevölkerung infolge fortschreitender Modernisierung - somit sei das revolutionäre Potenzial beschränkt. Vgl. Tehran \#A-708, 17.6.64, SOC 12-1 IRAN, CF 64-66.

${ }^{122}$ Spain Memo, 24.1.66, ,POL 12 Political Parties (general) 1966', Lot 70 D 330, NEA/IRN: 1964-66, Box 18.

${ }^{123}$ Tehran \#A-105, 17.8.65, POL 2 IRAN, CF 64-66.
} 
Annäherung an Moskau gedacht. Versuche der Auslands-Tudeh, erneut ein eigenes Netzwerk innerhalb Irans zu errichten, scheiterten an der Repression und der erfolgreichen Penetration der indigenen kommunistischen Gruppen durch den SAWAK. ${ }^{124}$ Xalil Maleki, bekannter Intellektueller und Anführer einer kleinen sozialistischen Abspaltung von der Tudeh, wurde gemeinsam mit einigen Parteigenossen 1966 in einem Schauprozess zu mehreren Jahren Haft verurteilt. ${ }^{125}$ Die Special Group (CI) hatte bereits Mitte 1965 registriert, das Regime setze nun verstärkt auf „tight control“ der oppositionellen Elemente und die immer wieder angekündigte Rückkehr zum in der Vergangenheit vorherrschenden gesteuerten Pluralismus werde wohl nicht vollzogen. ${ }^{126}$ Im Anschluss an die Ermordung des Ministerpräsidenten im Januar 1965 ersetzte der Schah den SAWAK-Direktor Pākrawān durch den bisherigen Leiter der nationalen Polizei, General Ne'mat-ollāh Nassiri. Die Botschaft wertete den Personalwechsel weniger als Resultat des Mansur-Attentats, sondern als bewussten Wandel der Ausrichtung der SAWAK ,[...] from Pakravan's relatively soft and improvising approach to the more efficient and possibly ruthless one of Nasiri. “127 Pākrawān war in seiner umsichtigen und grundsätzlich auf Überzeugung setzenden Art selbst bei der Opposition geachtet und ein Repräsentant des fast schon klassisch zu nennenden und spezifisch iranischen Stils der ,gemäßigten Repression“. Nassiri dagegen, ebenfalls Veteran des Mosaddeg-Coups, hatte sich in den Unruhen vom Juni 1963 als besonders rücksichtslos ,ausgezeichnet“. Beide wurden nach der Revolution hingerichtet. ${ }^{128}$ Das tatsächliche Ausmaß der Repression und auch die Zahl politischer Gefangener in dieser Zeit waren allerdings auch zeitgenössisch umstritten. ${ }^{129}$

Die Umsetzung des Reformprogramms in dieser Zeit gemeinsam mit der sich verschärfenden Repression hatten den Effekt einer weitgehenden Schwächung der bisherigen Opposition. Diese machte sich - zumindest im Vergleich mit den Krisenjahren von 1958 bis 1963 - kaum noch bemerkbar. Die verbliebenen Vertreter des ehemaligen pro-amerikanischen Netzwerks

\footnotetext{
${ }^{124}$ Vgl. Tehran \#A-709, 23.4.66, POL 29 IRAN, CF 64-66; bereits im Januar waren 13 Todesurteile in absentia gegen die im Ostblock lebende Führungsriege der Tudeh verhängt worden. Vgl. BEHROOZ, Rebels with a Cause, $38 \mathrm{ff}$.

${ }^{125}$ Maleki wurden Beteiligung an den 1963er-Unruhen und Kontakt zu Beijing vorgeworfen. Die US-Offiziellen schenkten diesem Prozess vergleichsweise hohe Aufmerksamkeit, offenbar auch von früherer geheimdienstlicher Nähe zu den Ex-Kommunisten rund um Maleki herrührend. Vgl. Tehran \#A-653, 26.3.66, POL 29 IRAN; Tehran \#A-232, 29.10.66, POL 12-6 IRAN, CF 64-66; offenbar stand Maleki auch bis Mitte 1965 im Kontakt zu den Israelis. Vgl. Rassias Memo, 17.3.66, Asnād 23, 4, S. 14; vgl. KatOUZIAN, Khalil Maleki, S. 168, $184 \mathrm{f}$. ${ }_{126}$ Memo for the Special Group (CI) 28.4.65, ,SPECIAL [...]6/3/65', Lot 68 D 451, SG (CI) Records, Box 8.

127 Tehran \#809, 30.1.65, POL 15-1 IRAN, CF 64-66; Pākrawāns Ehfrau erinnerte sich später, ihr Ehemann habe sich selbst als den einzigen Offiziellen in der Geschichte Asiens bezeichnet, der auf Folter verzichtete. Vgl. PAKRAVAN, Memoirs S. 26f.; sein Vorgänger Baxtijār hatte angeblich einen wilden Bären zur Einschüchterung von Gefangenen benutzt. Siehe die Behauptung des MI6-Veteranen YounG, Who is my Liege?, S. 9; zu Pākrawān und Nassiri vgl. BILl, Eagle and Lion, S. 162; Milani, Eminent Persians: I, S. 474-82, 468-73.

${ }^{128}$ William Polk lobte Pākrawān als ,,probably the most intelligent, urbane cop in the world.“; Polk Letter, 23.4.65, ,Near and Middle East 1965-66', Lot 72 D 139, S/P Records, Subject and Country: 1965-69, Box 315; CIA Station Chief Yatsevitch zufolge war Pākrawān ein ,,intellectual“" und ,academically inclined“ und eigentlich ungeeignet gewesen: „And, in fact, I think he found it very hard to carry out some of the functions that SAVAK was obliged to carry out."; Gratian Yatsevitch OHI, 5.11.88, OHIC, S. I-71; zu den Umständen seiner Entlassung vgl. Fatemeh Pakravan OHI, 3.3.83, IOHP, Transcript 2, S. 2-6; vgl. auch Memcon, 19.4.78, DNSA: IR01362.

129 Ein italienischer Weltbankmitarbeiter hatte die Zahl von 200.000 Gefangenen in sogenannten Umerziehungslagern von einem SAWAK-Offizier erfahren. Vgl. MemCon, 31.8.77, Asnād 7, S. 227; ein USDiplomat deutet in seinen semi-fiktiven Memoiren einen politischen Mord an einem kurdischen Dissidenten an, angeordnet vom Schah-Vertrauten 'Alam. Vgl. PRECHT, A Diplomat's Progress, S. 85-102.
} 
sahen sich neuem Druck seitens des Schah-Regimes ausgesetzt. Nach seiner Rückkehr nach Iran im Januar 1963 war 'Ali Amini in einer Audienz bereits vom Schah indirekt vor weiteren politischen Aktivitäten gewarnt worden. Nachdem sich Amini trotzdem um den Aufbau einer Koalition bemüht hatte, reagierte das Regime mit juristisch zweifelhaften Ermittlungen wegen Untreue gegen den ehemaligen Ministerpräsidenten und seine Ehefrau. Der SAWAK-Direktor warnte Amini persönlich vor einer Fortsetzung seiner oppositionellen Tätigkeiten. Amini aber kritisierte öffentlich die Umstände der Mağleswahlen im September 1963. Gleichzeitig führte er Verhandlungen mit Vertretern der Zweiten Nationalen Front über eine mögliche gemeinsame Koalition - ähnliche Verhandlungen waren bereits während seiner Amtszeit gescheitert. ${ }^{130}$ Nach 1963 aber war diese Art der „loyalen Opposition“, der Positionskämpfe zwischen politischen Prätendenten außerhalb der direkten Kontrolle des Schahs und der Sicherheitsorgane, die zuvor noch charakteristisch für das System des gesteuerten Pluralismus in Iran waren, nicht mehr erwünscht. ${ }^{131}$ Statt der erhofften Ernennung zum Premier wurde Amini im Mai 1964 ein weiteres Mal zur Ausreise genötigt und auf diese Weise politisch „kaltgestellt“. ${ }^{132}$ Gerüchte über amerikanische Kontakte zum ehemaligen Premier Anfang 1968 sorgten später erneut für Verstimmungen im amerikanisch-iranischen Verhältnis. Den USA gelang es den Schah zu überzeugen, dass es keine solchen Kontakte gegeben habe. ${ }^{133}$ Der frühere Landwirtschaftsminister Arsanğāni, für viele weiterhin der eigentliche „Held“ der Landreform, wurde im September 1964 als Botschafter in Italien abgesetzt, nachdem er in öffentlichen Schriften die Wirtschaftspolitik der Mansur-Regierung kritisiert hatte. ${ }^{134}$ Anfang 1964 war bereits die erneute Verhaftung von General Garani gemeinsam mit acht hochrangigen ehemaligen und aktiven Offizieren erfolgt. Angeblich gab es Hinweise, wie Mitglieder des ehemaligen pro-amerikanischen Netzwerks der Botschaft berichteten, dass auch Amini und Arsanğāni in diese angebliche Verschwörung verwickelt waren. ${ }^{135}$

In der Nationalen Front zeigten sich Auflösungserscheinungen. Intern wurde die Führung der

\footnotetext{
${ }^{130}$ Aminis „Koalition“ bestand aus ehemaligen politischen Weggefährten wie Deraxšeš, Teilen der Geistlichkeit, und Vertretern des Basars. Der Schah empfand offenbar eine große Antipathie für Amini selbst. Vgl. Tehran \#A115, 20.8.63, POL 12 IRAN; Tehran \#A-209, 1.10.63, POL 15 IRAN, CF 63; Lorenz Memo, 20.5.64, [ohne Folder], Lot 69 D 225, NEA/IRN: 1964-66, Box 6.

${ }^{131}$ Vgl. Lorenz Memo, vorherig. Anm.

132 Zurück in Iran, setzte Amini Anfang 1965 seine Bemühungen um eine Annäherung an die Nationale Front fort. Vgl. Lorenz Memo, 6.5.64, ,POL 12-c Other Political [...]', Lot 69 D 225, NEA/IRN: 1964-66, Box 6; Tehran \#A-50, 25.5.64, POL 6 IRAN; Tehran \#1012, 8.5.64, POL 7 IRAN, CF 64-66; MemCon, 13.2.65; Miller Memo, 4.2.65, ,POL 12-c Other Political [...]', Lot 69 D 489, NEA/IRAN: 1964-66, Box 11.

${ }_{133}$ Auslöser war eine arglose Bemerkung Meyers zu einer Verwandten Aminis auf einem Empfang. Vgl. State \#121476/\#124570/\#126616, 28.2./5.3./8.3.68, FRUS 64-68, XXII, S. 464f., 466ff., 477ff.; der Schah erklärte seinen Protest später mit dem gleichzeitigen Auftreten von Protesten unter den Studenten Teherans, ,[...] which resembled developments in 1961 prior to Amini's coming to power", ein weiterer Beleg für Mohammad Rezās Überzeugung, dass die Ereignisse von 1961 von den USA inszeniert worden waren, um Amini als Premier durchzusetzen. Vgl. Tehran \#3627/\#3767, 6./14.3.68, ebd., S. 474f., 479ff.; Tehran \#3681, 9.3.68, , Iran [...] II [1 of 2]; 1/66-1/69', NSF, Country File, Box 136, LBJL; vgl. MEYER, Quiet Diplomacy, S. 138f.; auch die Briten waren sich der langfristigen Belastungen des amerikanisch-iranischen Verhältnisses infolge der früheren Unterstützung Aminis bewusst: ,the Shah certainly held it against them and they were in the dog-house for four years.“; zit. aus MURRAY, Rise and Fall, S. 155.

${ }^{134}$ Vgl. Tehran \#A-159, 5.10.64, POL 2 IRAN, CF 64-66.

135 Offenbar glaubte der britische Geheimdienst an eine reale Bedrohung des Regimes. Garani wurde im Juli zu drei Jahren Gefängnis verurteilt. Vgl. MemCon, 4.1.64, ,POL 23-9-a Coup Attempts Iran 1964‘, Lot 69 D 178, NEA/IRN: 1964-66, Box 4; Tehran \#621, 14.1.64, POL 23-9 IRAN, CF 64-66.
} 
Front scharf kritisiert für die vielen taktischen Fehler seit 1960. Allāhjār Sāleh, im Herbst 1963 mit außerordentlichen Vollmachten für die Erneuerung der Front ausgestattet, versuchte offenbar den bisherigen antagonistischen Kurs gegenüber dem Regime abzumildern, um wenigstens begrenzte Aktivitäten der Front $\mathrm{zu}$ ermöglichen. Gegen diese eher kompromissbereite Haltung setzten sich vorwiegend jüngere Kader zur Wehr, die sich zudem langsam nach dem Muster der globalen Protestbewegungen der 1960er Jahre radikalisierten hier lag der Ursprung des Stadt-Terrorismus der 1970er Jahre in Iran. Mosaddeg unterstützte aus seinem Hausarrest heraus die jüngeren Aktivisten und kritisierte in offenen Briefen Sāleh und die alte Führung der Front für ihre Passivität und den Ausschluss anderer Oppositionsgruppen. Im Laufe des Jahres 1964 löste sich die Zweite Nationale Front ohne eigentlichen Beschluss auf - eine von Mosaddeg selbst betriebene Neugründung einer Dritten Nationalen Front 1965 scheiterte bald an Repressionsmaßnahmen des Regimes und am ideologischen Wandel in der Opposition Irans, mit neuen Schwerpunktsetzungen auf religiöse einerseits oder marxistisch-maoistische Inhalte andererseits. ${ }^{136}$

Die schleichende Radikalisierung innerhalb der Nationalen Front, gerade bei den jüngeren Kadern, war symptomatisch für eine Verlagerung oppositioneller Aktivitäten weg von einer Nutzung des immer begrenzter werdenden Spielraums in Parlament und Öffentlichkeit und hin zu Untergrundorganisation einschließlich bewaffneten Widerstands, Attentaten und Terrorismus. ${ }^{137}$ Angesichts der Reputation der älteren Frontführung und deren eher bürgerlich anmutenden Mangels an Risikobereitschaft fiel es amerikanischen Beobachtern anfangs schwer, diese Tendenzen allzu ernst zu nehmen. ${ }^{138}$ Zwar war diese Einschätzung mit Bezug auf die alten Kader zutreffend. Allerdings bildeten sich jenseits der Wahrnehmung der Botschaft bereits radikalisierte Gruppierungen, vor allem im linken Spektrum der Nationalen Front bzw. innerhalb der diversen marxistisch orientierten Kleingruppen. Die Abspaltung einer maoistischen Gruppe von der Tudeh im Februar 1964, die sich später „Revolutionäre Organisation der Tudeh-Partei Irans“ (ROTPI) (sāzmān-e enġelābi-je hezb-e tude-je irān) nannte, war ein erstes Zeichen für die einsetzende Fragmentierung der Oppositionsgruppen und die Anfänge bewaffneter Guerilla-Aktionen gegen die Schah-Herrschaft und bald auch gegen

\footnotetext{
${ }^{136}$ Vgl. KatouZIan, Struggle for Power, S. 240-51; die CIA berichtete, die Gruppe der jüngeren radikaleren Aktivisten sei vom SAWAK penetriert worden. Führungsfiguren in der nationalistischen Opposition gestanden 1964 ein, dass die religiös-oppositionelle „Freiheitsbewegung Irans“ Mehdi Bāzargāns inzwischen größere Unterstützung in der Bevölkerung genieße als die Front selbst. Die Überflügelung der säkularen Nationalisten durch religiös-konservative Opposition war bereits hier absehbar. Vgl. Miller Memos, 15./18.4./26.5.64, , POL 12d National Front IRAN 1964", Lot 69 D 225, NEA/IRN: 1964-66, Box 6; der wichtigste Vertreter der jungen Radikalen in der Front, Šāpur Baxtijār, plädierte laut einem CIA-Bericht für Zusammenarbeit mit Basar und Geistlichkeit im Kampf gegen die Regierung. Vgl. CIA Field Information Report, 2.3.64, Asnād 20, 1, S. 71; zur Radikalisierung vgl. RAMAZANI, Patterns of Influence, S. 80-90.

${ }^{137}$ Es war wieder einmal der Iranexperte Bill Miller, der vor langfristigen Konsequenzen warnte. Die nächste Generation von Intellektuellen und Studenten, so Miller, sei weitaus stärker als ihre Vorgänger marxistischen und maoistischen Ideen zugeneigt - die Botschaft kommentierte trocken: „We have heard so much of late about this alleged leftward drift but have seen little hard evidence of it.“; Herz Letter, 31.8.65; Miller Memo, o.D., ,POL 2 General Reports Iran 1965', Lot 69 D 490, NEA/IRAN: 1964-66, Box 12; zur Radikalisierung der Exilopposition siehe SADEGHI-BOROUJERDI, Iran's Tri-Continental Movement.

${ }^{138}$ Vgl. MemCon, 19.5.65; Herz Letter, 5.4.65, ,POL 12-d National Front Iran 1965`, Lot 69 D 489, NEA/IRAN: 1964-66, Box 11 .
} 
die amerikanische Präsenz in Iran. ${ }^{139}$ Die Versuche der ROTPI, eine Bauernrevolution auf dem Land zu initiieren bzw. den Stammesaufstand in Fārs für eigene revolutionäre Zwecke zu instrumentalisieren, scheiterten jedoch kläglich. 1965 konstituierten sich auch die „Volksmudschahedin“ (sāzmān-e moğāhedin-e xalg̀-e irān) mit ihrer idiosynkratischen Synthese islamisch-schiitischen und marxistisch-leninistischen Gedankenguts. ${ }^{140}$ Der eigentliche bewaffnete Guerilla-Kampf gegen Mohammad Rezās Herrschaft wird gewöhnlich auf das Jahr 1971 und die Gründung der „Organisation der Guerillas der iranischen VolksFedajin“ datiert. ${ }^{141}$ Die „Volks-Fedajin“ nahmen den bewaffneten Kampf umgehend auf, die Volksmudschahedin folgten alsbald diesem Beispiel. Zwar hatte man im Anschluss an das Attentat auf den Schah 1965 das Unruhepotential gerade unter der jungen Generation erkannt und ein begrenztes Programm gerichtet auf die iranische Jugend in Gang gebracht, die Gefahr gewaltsamen Widerstands aber durchweg unterschätzt. ${ }^{142}$ Erst in der Retrospektive, im Sommer 1972, konstatierte eine Einschätzung von INR im State Department, dass ,[...] a violence-inclined 'youth underground' has taken root in Iran with possibly serious consequences for the country's long-term stability. “143

Mitte der 1960er Jahre zeigte man sich innerhalb der US-Botschaft von den ersten Signalen für die Entstehung eines solchen bewaffneten Untergrunds noch wenig beunruhigt. Im Gegenteil, der augenscheinliche Zerfall der Opposition, die kaum noch wahrnehmbaren öffentlichen Unmutsäußerungen, die zunehmende Effizienz der Sicherheitsorgane und insbesondere des SAWAK, all dies wurde letztlich als Erfolg der Konsolidierung der Schah-Herrschaft interpretiert. Die wenigen verbliebenen oppositionellen Kräfte, die sich zumindest potentiell in der Zukunft in ernsthafte Bedrohungen für das Regime entwickeln könnten, seien durch eine Mischung aus Überzeugungsarbeit, Repression und politischem Geschick ausmanövriert worden. Ende 1965 wurde dem alten Wunsch NEAs entsprochen und Iran von der Liste der „kritischer Länder“ für die kontinuierliche Behandlung durch die Special Group (CI) gestrichen. Die Botschaft bestätigte gegenüber der Special Group (CI) im April 1965 in einem CIDP-Progress Report über die interne Sicherheitslage denn auch ein weiteres Mal die Einschätzungen der Vorjahre, dass ,[....] no clearly identifiable threat to internal security is likely to develop in the near future except in the event of the demise or abdication of the

\footnotetext{
139 Zur ROTPI vgl. Vgl. BEHROOZ, Guerrilla Movement, S. 194f.; ders., Rebels with a Cause, $39 \mathrm{ff}$.

${ }^{140}$ Vgl. ABRAHAMIAN, Iranian Mojahedin, S. 85-126; bereits Anfang der 1980er Jahre identifizierte die CIA die Bedeutung der Gruppe als schlagkräftige Opposition gegen die Herrschaft Khomeinis. Vgl. CIA, National Foreign Assessment Center Intelligence Assessment, , Iran: The Mujahedin \{fehlender Teil des Titels klassifiziert\}, August 1981, CREST: \#RDP06*001-7.

${ }^{141}$ Vgl. BeHROOZ, Rebels with a Cause, S. 48-70; zu den kaum zu überschauenden internen Konflikten zwischen den Gruppen und den ideologischen Auseinandersetzungen der extremen Linken vgl. VAHABZADEH, Guerrilla Odyssey, S. 16-77.

${ }^{142}$ In der Special Group (CI) wurde im Mai 1965 eine Schwerpunktverlagerung auf Youth-Programme und eine Einbeziehung Irans durch das Interdepartmental Youth Committee angeregt. Vgl. Meeting Special Group (CI), 20.5.65, FRUS 64-68, XXII, S. 152ff.

${ }_{73}^{143}$ INR Research Memo RNAN-18, ,Internal Dissidence - A Note of Warning‘, 12.6.72, POL 23-8 IRAN, CF 7073.
} 
Shah."144

Angesichts der Machtkonzentration in der Hand des Monarchen und des Schwindens wenigstens begrenzt autonomer Machtzentren innerhalb Irans war dies die eigentliche Hauptsorge aus amerikanischer Sicht, vielleicht sogar zu diesem Zeitpunkt die letzte verbliebene Sorge. Bereits seit den Unruhen vom Sommer 1963 hatte man mit einer Wiederkehr von Attentatsversuchen gegen führende politische Persönlichkeiten und insbesondere gegen Mohammad Rezā selbst gerechnet, in dem Bewusstsein, dass es derartige Phasen in der iranischen Geschichte wiederholt gegeben hatte. Allerdings sah man die Gefahr nicht in der seitens der Botschaft und der CIA unterschätzten, gerade geschilderten Neuorganisation und Radikalisierung der Opposition. Für wahrscheinlicher hielt man das Erscheinen fanatisierter Einzeltäter. Die Ermordung des Ministerpräsidenten Mansur im Januar 1965 schien diese These zu bestätigen. Zwar sprach der Schah in der Öffentlichkeit von einer „unheiligen Allianz“ zwischen Kommunisten und Reaktionären, welche die Tat zu verantworten habe. Allerdings handelte es sich bei dem Täter um einen 20-jährigen Fanatiker, der einer kleinen religiösen Gesellschaft angehörte. Zwar hatte es anfangs Gerüchte über Verbindungen zwischen der Gruppe und dem inzwischen exilierten Ājatollāh Khomeini gegeben, doch bewahrheiteten sich diese nicht. Offenbar handelte die Gruppe eigenständig für INR im State Department war diese Entwicklung jedoch auch Ausdruck für die breitere Unzufriedenheit in der Bevölkerung, gerade bei den frommeren Unterschichten. ${ }^{145}$ Gegen die Verwendung der Beschreibung ,widespread discontent“" wehrte sich GTI jedoch sofort: „In our judgment political dissidence in Iran in the mid-sixties is at relatively low point, viewed against the experience of the past fifty years. “146

Weitaus alarmierender aus amerikanischer Sicht war das Attentat auf Schah Mohammad Rezā im April 1965. Ein Mitglied der Palastwoche, bewaffnet mit einer Maschinenpistole, versuchte zum Schah selbst vorzudringen, was ihm beinahe gelungen wäre. Der Attentäter und zwei weitere Wachen kamen bei dem Anschlag ums Leben. Offenbar handelte es sich nicht um einen Einzeltäter, sondern um eine von einer größeren Gruppierung geplanten Tat. Dieses Mal kamen die Täter nicht aus dem religiösen Milieu. Es handelte sich vielmehr um eine Gruppe von Studenten, die gemeinsam in Großbritannien die Universität besuchten, und ideologisch der bereits genannten ROTPI, der maoistischen Abspaltung der Tudeh, zuneigten. ${ }^{147}$ Meyer

\footnotetext{
144 Jernegan Memo, 28.4.65, ,Special Group (CI) 4/29/65-6/3/65‘, Lot 68 D 451, SG (CI) Records, Box 8; auch die vorhergehenden Progress Reports vom 14.10.63 und 2.3.64 hatten eben diese Wendung enthalten.

${ }^{145}$ Vgl. Howison Memo, 21.1.65; Hughes Memo, 28.1.1065, FRUS 64-68, XXII, S. 126, 127f.; Note ,Khomeini and Mansur', 22.1.65, ,POL 23-8 [...]65', Lot 69 D 489, NEA/IRN: 1964-66, Box 11.

${ }^{146}$ Bracken Memo, 10.2.65, ,POL 23-8 Demonstrations. [...]65', Lot 69 D 489, NEA/IRAN: 1964-66, Box 11; Meyer bestätigte die Gesamteinschätzung im Herbst 1966: „It has been years since the political atmosphere has been as sluggish, as self-satisfied, or as resigned to the status quo."; Meyer Letter, 22.10.66, FRUS 64-68, XXII, S. 321 .

${ }^{147}$ Vgl. Jernegan Memo, 10.4.65, ebd., S. 138; Tehran \#1115/\#1212, 11./28.4.65, POL 15-1 IRAN, CF 64-66; vgl. VAHABZADEH, Guerrilla Odyssey, S. 7f.; Richter Douglas, während der Kennedy-Zeit emsiger Vorkämpfer für eine Wende in der amerikanischen Iranpolitik, setzte sich in einem Brief an Präsident Johnson für die verurteilten maoistischen Studenten ein. Von den 14 Angeklagten wurden zwei zum Tode, einer zu lebenslanger Haft und die
} 
beschwichtigte sogleich aus Teheran, trotz des Attentats habe sich die grundsätzliche Einschätzung der Sicherheitslage nicht verändert. Allerdings habe der Anschlag die Tatsache herausgestellt, ,[...] that stability in Iran is unduly dependent on the life of one man. "148 Taten fanatischer Einzeltäter seien kaum zu verhindern und eine Wiederholung eines solchen Versuchs in der Zukunft durchaus wahrscheinlich. Insgesamt aber hätten die Sicherheitskräfte die Lage weiterhin unter Kontrolle. ${ }^{149}$ Die CIA sekundierte mit ihrer Einschätzung der Lage als ,,a picture of relative peace and security“. Die Opposition sei ,,[...] so disorganized and fragmented as to be powerless.“" ${ }^{50}$ Allerdings habe der Attentatsversuch illustriert, wie gefährlich die Lage nach einem unerwarteten und plötzlichen Abgang Mohammad Rezās werden könne. Da es keine Pläne für ein solches Szenario gebe, sei für einen solchen Fall mit einer Periode des Chaos zu rechnen, die wohl am wahrscheinlichsten durch die Errichtung einer Militärdiktatur bereinigt werden würde. In der Special Group (CI) mahnte DCI Raborn die Aktualisierung entsprechender Notfallpläne für eine solche Eventualität auf US-Seite an. ${ }^{151}$

Im Mai 1966 wurde intern eine aktualisierte Version des alten Contingency Paper von 1963 verabschiedet, doch ist keiner der beiden Pläne in den Akten auffindbar. ${ }^{152}$ Eine

meisten anderen zu kürzeren Haftstrafen verurteilt. Der Schah wandelte die Todesstrafen in lebenslange Haft um. Bereits 1971 begnadigte der Schah die Mitglieder der Gruppe. Vgl. Komer Letter, 27.11.65, ,Iran, Memos [...]12/65', NSF, Country File, Box 136, LBJL; Douglas Letter, 8.11.65; Howison in NEA beschwerte sich insbesondere über einen Satz in einem Briefentwurf an Douglas, der die US-Politik beschrieb als, ,continuing effort $[\ldots]$ to do all they can to raise the level of basic rights and freedoms throughout the world."; Howison kommentierte scharf, diese Passage sei ,[...] inconsistent with my understanding of USG policy.“; Komer Draft Letter, o.D., encl. to Howison Memo, 23.12.65, ,POL 23-8 Assassination [...]65', Lot 69 D 489, NEA/IRN: 1964-66, Box 11; vgl. BILL, Eagle and Lion, S. 162f., 175f.; zur Mobilisierung der iranischen Studentenschaft im Ausland infolge der Anklage gegen die Attentäter vgl. SHANNON, Losing Hearts and Minds, S. 141-54.

${ }^{148}$ Tehran \#1171, 20.4.65, FRUS 64-68, XXII, S. 140; neben einem geglückten Attentat befürchtete man von amerikanischer Seite fortwährend ein unzeitiges Ableben Mohammad Rezās infolge seiner (nicht vorhandenen) Flugkünste. Vgl. Rockwell Letter, 24.3.64, ,DEF-Defense Affairs 1-1 [...]64‘, Lot 69 D 95, NEA/IRN: 1964-66, Box 3; angesichts der einseitigen Abhängigkeit der eigenen Position vom Monarchen wuchs das ohnehin große Interesse am persönlichen Gesundheitszustand Mohammad Rezās, ein Faktor, der in der Zukunft noch wichtiger werden sollte. Die CIA berichtete 1964 von kleineren Gebrechen und anhaltendem Stresszustand. Insgesamt sei die Gesundheit Mohammad Rezās aber zufriedenstellend. Besorgnis erregten immer wieder längere Kuraufenthalte des Schahs in Europa. Vgl. Herz Letter, 18.2.64, ,POL 8 Neutralism [...]64‘, Lot 69 D 225 , NEA/IRN: 1964-66, Box 6.

${ }^{149}$ Ebd.; in einem Treffen des State Department mit den JCS bestätigte auch Botschafter Holmes, dass, , $\{\mathrm{t}\}$ he one great danger to continuing progress and stability is that some one will kill the Shah."; State-JCS-Meeting, 23.4.65, FRUS 64-68, XXII, S. 143.

${ }^{150}$ Current Intel Memo OCI No. 1109/65, 23.4.65, ebd., S. 141.

${ }^{151}$ Vgl. Meeting Special Group (CI), 20.5.65, ebd., S. 152ff.; die Nachfolge war seit der Geburt des ersten Sohns Kuroš Rezā Pahlawi am 31. Oktober 1960 und des zweiten Sohns 'Ali-Rezā am 28. April 1966 gesichert. Ersterer ist heute nominelles Oberhaupt der Familie, letzterer schied 2011 durch Freitod aus dem Leben. Laut Thronfolgeregelung wäre nach den beiden Söhnen die Linie des 1954 verstorbenen, jüngeren Bruders des Schah, 'Ali Rezā, nachgefolgt. Nach dessen Tod erst war dessen Ehe mit Christine Cholewsky bekannt geworden, aus der 1947 der Sohn Patrick "Ali hervorging, der - die Botschaft nannte dies eine „genealogical oddity“ - bis 1960 theoretisch der glaubwürdigste Prätendent war. Er wurde 1975 aufgrund seiner öffentlichen Kritik am Regime interniert und lebt seit der Revolution als Autor spiritueller Erbauungsliteratur im Exil. Da Kuroš Rezā keine Söhne hat, wären Patrick 'Ali bzw. dessen aus einer Ehe mit einer Schweizerin hervorgegangenen Söhne im Falle seines Ablebens die Thronfolger. Die Halbbrüder Mohammad Rezās waren von der Thronfolge aufgrund der Verfassung ausgeschlossen. Vgl. Tehran \#A-328, 19.12.66, POL 15-1 IRAN, CF 64-66; die CIA berichtete 1957 über eine Anweisung des Schahs, Patrick 'Ali zum legitimen Nachfolger seiner selbst zu ernennen. Vgl. Staff Paper, o.D., ,Iran (2)', WHO, NSCS, Special Staff File Ser., Box 4, DDEL; zu Patrick 'Ali vgl. MEIER, Helvetiens guter Draht, S. $228 \mathrm{ff}$.

${ }_{152}$ Zur Contingency-Studie vom Oktober 1963 vgl. Rockwell Letter, 20.1.64, ,DEF - DEFENSE AFFAIRS 1-1 [...]64', Lot 69 D 95, NEA/IRN: 1964-66, Box 3; Meyer Letter, 23.2.66; Hare Letter, 19.5.66, ,Iran', Lot 80 D 234, ASS/NEA: Top Secret Records 1965-75, Box 1; auf Anraten der Botschaft gab es im Contingency Paper keine Festlegung auf einen amerikanischen Favoriten für die Regentenrolle, zumal der eigene Favorit nun ,retired“ sei. Leider ist die Identität dieser Person nicht geklärt. Für eines der Szenarien hatte man einen speziellen Ordner 
Nachfolgeversion von 1970 aber definierte dezidiert das Hauptproblem des amerikanischiranischen Verhältnisses: ,[...] that so much depends on one man. “153 Interessant aus Sicht der perzipierten Einflussmöglichkeiten Washingtons in Iran nach dem weitgehenden Verlust des pro-amerikanischen Netzwerks und der Aufgabe der permanenten Intervention ist die Feststellung im 1970er-Plan, im Falle einer Ermordung bzw. Amtsenthebung Mohammad Rezās sei zumindest mittelfristig nicht mit der Etablierung einer durchsetzungsfähigen proamerikanischen Regierung zu rechnen. Offenbar anerkannte man zu dieser Zeit in Washington, dass eine Wiederholung eines Coups nach dem Muster von 1953 im veränderten Iran der 1970er Jahre unmöglich wäre. ${ }^{154}$ Ein Einsatz amerikanischer Militärmacht zur Durchsetzung der eigenen Interessen, selbst auch nur zum Schutz amerikanischer Bürger und Diplomaten innerhalb Irans, sei in einem solchen Ernstfall eher unwahrscheinlich, so das Papier. Wenngleich einige der Annahmen auf bürokratische Kritik stießen, so veranschaulichten diese Pläne dennoch, wie bewusst man sich intern der eigenen Abhängigkeit von der Person Mohammad Rezās war. ${ }^{155}$ Die Unsicherheit über die Folgen eines überraschenden Ablebens des Monarchen reduzierte Mohammad Rezā im September 1967 teilweise mit der Einrichtung eines Regentschaftsrats durch einen Verfassungszusatz. Sollte er demnach vor dem 31. Oktober 1980, also dem 20. Geburtstag des Thronfolgers, versterben, würde die Šahbānu bis zu diesem Tag einem Regentschaftsrat vorstehen, der die Geschicke des Landes bis zur Volljährigkeit von Kuroš Rezā bestimmen sollte. Die Einrichtung des Regentschaftsrats etablierte zumindest ein

im Büro des Botschafters vorbereitet, der 25 Kopien der relevanten Artikel der iranischen Verfassung bezüglich der Thronfolge sowie „locator sheets“" der wichtigsten iranischen Persönlichkeiten enthielt, um diese im Falle einer Nachfolgekrise möglichst rasch lokalisieren und informieren zu können. Zudem hatte man von den Iranern unabhängige Kommunikationseinrichtungen etabliert, was in der späteren Geiselkrise während der iranischen Revolution noch von großer Wichtigkeit werden sollte.

${ }^{153}$ Contingency Plan:, Proposed Action by the United States in the Event of the Death of the Shah', o.D., ebd.; ein Memorandum vom Oktober 1972 erwähnt eine generische Version der früheren Pläne aus dem Jahr 1970. Diese ist im gleichen Folder enthalten, während die Pläne von 1963 und 1966 in den gesichteten Akten nicht enthalten waren. Vgl. Michaud Memo, 3.10.72, ebd.

154 „Not only may we not hope to determine the final shape of an emergent government for the complications and uncertainties of contemporary Iran are too great for us to do this, but, even could we ordain a responsible government, there is little guarantee that such a government could remain both in office and responsive to our wishes."; vgl. Proposed Action, vorherig. Anm.

${ }^{155}$ IRN im State Department kritisierte die Annahme, dass ,the U.S. will have virtually no chance to influence events after the Shah departs" als „too pessimistic“. Auch sah man eine militärische Involvierung in mögliche Nachfolgewirren als durchaus nicht unwahrscheinlich an. Vgl. Childs Memo, 12.8.70, ebd.; andere Stimmen sahen im Fall des Tods oder der Absetzung Mohammad Rezās den Schwagers des Schahs und Kommandeur der Luftwaffe, General Mohammad Xātami, als wahrscheinlichen „Strong Man“ an. Vgl. AzIMI, Quest for Democracy, S. 236; ein Führer der Nationalen Front diskutierte mit einem US-Offiziellen ebenfalls die verschiedenen Szenarien im Anschluss an eine Ermordung Mohammad Rezās. Mahdawi sah dabei einen Zusammenbruch der öffentlichen Ordnung in Teheran und den Provinzen voraus. Zudem sei mit einer raschen Rückkehr der Tudeh zu alter Stärke und Putschversuchen ehemals mächtiger Offiziere wie Baxtijār zu rechnen. Khomeini sei allerdings kein Faktor: ,[...] he would go to Qom and would not be a major factor in the Tehran situation.“; MemCon, 7.5.65, ,POL 2 - General Reports Iran 1965', Lot 69 D 490, NEA/IRN: 1964-66, Box 12; der Halbbruder des Schahs, Prinz 'Abdorrezā, äußerte sich 1964 pessimistisch über die generelle Entwicklung in Iran und das diktatorische Verhalten seine Bruders - sollte es zur Krise kommen, könne er aufgrund seiner Verantwortung für das Land keine neutrale Haltung einnehmen, so der Prinz gegenüber einem nicht genannten Gesprächspartner. Vgl. Memo for the Record 11.6.64, ohne Folder, Lot 69 D 225, NEA/IRN: 1964-66, Box 6; zugleich bemühte man sich seitens der Johnson-Administration um vertiefte Informationen über das Weltbild des jungen Kronprinzen Rezā Pahlawi. Zwar wisse man, dass der fünfjährige Prinz LBJ-Cowboystiefel trage und auch JFK-Malbücher benutze, doch sei seine Einstellung zu den USA unbekannt. Man kontrolliere bereits die Quelle für die Geschenke des Vaters, erwartungsgemäß Kriegsspielzeug, doch habe man keine Kontrolle über die von der Mutter geschenkten Spielzeuge. Man gedachte zudem ihm über die Präsidentenfamilie entsprechendes Spielwerk zukommen zu lassen ,,to direct his thinking along economic development lines.“; Vorschläge waren u.a. SpielzeugBagger oder Baukästen. Vgl. Note ,Pahlavi, Reza', o.D. [vermutlich nach Oktober 1965], ,EDX 15 Foreign Leader Program Iran 1965', Lot 69 D 426, NEA/IRN: 1964-66, Box 8. 
formelles Verfahren für die Nachfolge, wobei die Sorge auf Seiten der USA vor einem möglichen Chaos infolge des Todes des Schahs nur gemildert, aber keineswegs beseitigt war. ${ }^{156}$

Angesichts der inzwischen einseitigen Bindung der Westorientierung Irans an die Person des Schahs war das grundlegende Verständnis des sich in Iran vollziehenden beschleunigten Wandels und dessen Folgen umso bedeutender. Den zentralen Widerspruch registrierte man durchaus. So konstatierte das Contingency Paper lapidar:

The kind of modernization process which Iran is experiencing could under other political leadership be expected to produce a set of related political changes, but the Shah's competence at controlling Iran's politics and in using a traditional power apparatus to do modern things masks political ferment. ${ }^{157}$

Diese Divergenz zwischen zunehmend rasanter ökonomischer Entwicklung und sozialer Transformationen auf der einen und der politischen Stagnation auf der anderen Seite war auch für die Zeitgenossen augenfällig. Nur wenige amerikanische Offizielle aber waren bereit, sich eingehender mit der Substanz und den möglichen Folgen dieser Divergenz zu befassen, angesichts der tradierten amerikanischen Obsession mit der „Stabilität“ Irans im Grunde überraschend. ${ }^{158}$

Eine interne Bestandsaufnahme von GTI vom Oktober 1964 über mögliche Probleme in Iran in der nahen Zukunft definierte allerdings eben diese Divergenz als das zentrale Problem amerikanischer Politik in der Zukunft. Es stelle sich die Frage der eigenen Positionierung hinsichtlich einer politischen Öffnung in Iran. Die jüngsten Erfolge Mohammad Rezās, das Überstehen der Krisenjahre 1960-61 und der Sieg über die „,anti-modernization forces“ im Jahr 1963, und sein daraus resultierendes Selbstbewusstsein könnten zwei gegensätzliche Folgen zeitigen, so das Papier. Denkbar sei eine noch größere Entschlossenheit des Monarchen, die Macht weiter in seiner Person zu konzentrieren und die Zügel fest in der Hand zu halten oder, alternativ, eine Bereitschaft, angesichts der abnehmenden Bedrohungen für die PahlawiMonarchie wieder schrittweise Macht an andere Institutionen abzugeben und eine politische

\footnotetext{
${ }^{156}$ Vgl. INR Research Memo RNA-46, 9.10.67, FRUS 64-68, XXII, S. 431; Tehran \#A-218, 24.10.67, POL 15-1 IRAN, CF 67-69; vgl. auch LENCZOWSKI, Second Pahlavi Kingship, S. 438f.; im Regentschaftsrat vertreten waren der Premier, die Vorsitzenden der beiden Häuser des Mağles, der Oberste Richter des Verfassungsgerichts und vier weitere ,sachkundige“ Mitglieder, deren Identität nicht bekannt, aber offenbar vom Schah bereits festgelegt war. Vgl. CIA Memo, 27.1.75; CREST: \#RDP86*010-3; ein Botschaftsdokument aus dem Jahr 1975 sah für die Zukunft einen Machtkampf zwischen Farah und Ašraf voraus. Vgl. Tehran \#A-19, 28.1.75, Asnād 7, S. 130-44; vgl. auch Embassy Note, , The Succession Problem‘, o.D., ebd., S. 145-8; die CIA erwartete 1978 im Falle eines Ablebens des Schahs dominanten Einfluss Farahs und ihrer Vertrauten. Vgl. CIA, National Foreign Assessment Center Intelligence Assessment, ,Iran After the Shah', August 1978, CREST: \#RDP80*001-6.

${ }_{157}$ Proposed Action, S. 612, Anm. 153.

${ }^{158}$ Harold Saunders vom NSC warnte Komer bereits im Mai 1964, man setze im Kampf gegen den globalen Kommunismus zu stark auf ökonomische und militärische Hilfe: „We have no goals for political development, and we don't really understand the political uses of aid."; die 1960er seien die Dekade wirtschaftlicher Entwicklung - die 1970er ,[...] will prove the need for political development--too late.“; Saunders Memo, 20.5.64, ,AidDecember 1963, 64, 65, 66 [1 of 3]', NSF, FRWK, Box 10, LBJL; das Divergenz-Thema findet sich auch vereinzelt in der zeitgenössischen akademischen Literatur zu Iran. Vgl. z.B. RAMAZANI, Iran's White Revolution, S. $138 \mathrm{f}$.
} 
Modernisierung analog zu anderen Sektoren wie Wirtschaft und Gesellschaft zuzulassen. Aus amerikanischer Sicht wäre die Schwierigkeit zu meistern, an entscheidenden Wendepunkten den Schah bezüglich „optimum timing and degree of advisable adaptations“ zu beraten. ${ }^{159}$ Offenkundig aber war, dass man gedachte, sich bezüglicher politischer Öffnung an das vom Monarchen gewünschte Tempo anzupassen - Initiativen im Sinne der reformistischen Intervention der frühen 1960er Jahre kamen angesichts der geänderten Gesamtlage keinesfalls mehr in Frage.

Eine Debatte über die Folgen des raschen Wandels in Iran und seine Auswirkungen auf die amerikanische Politik fand in der Johnson-Administration nicht statt. Vorherrschend blieb das Bild von Iran als „bright spot“ in einer für US-Politik schwierigen bis mitunter feindseligen regionalen Umgebung. Angesichts der schwierigen Phasen seit Ende der 1950er Jahre erschien Iran für amerikanische Entscheidungsträger wie Analysten im State Department oder innerhalb der Intelligence Community mehr und mehr wie ein einzigartiger wie vorbildhafter Erfolgsfall. Nur wenige kritische Geister störten sich an dieser Interpretation, die die weiterhin bestehenden Probleme zu bagatellisieren schien. Wieder einmal war es William Polk vom Policy Planning Council, der sich jenseits der Oberflächlichkeiten mit der Thematik eingehend befasste. Im April 1965 informierte er den neuen Botschafter Armin Meyer über die Lage in Iran. Polk zufolge teilte die akademische Welt in den USA keineswegs die positive Sicht der US-Politik bezüglich Irans. Polk selbst glaubte Parallelen in den Transformationsprozessen der verschiedenen nahöstlichen Staaten zu erkennen und entwickelte eine Typologie von Entwicklung und gesellschaftlichen und politischen Wandel, um diese Prozesse - ,the political stresses which development produces“ - besser zu verstehen. Seine „Analysis of Social Change“ publizierte er einige Jahre später nach seiner Rückkehr in die akademische Welt. ${ }^{160}$

In seinem Papier warnte Polk vor simplifizierenden Annahmen der politischen Folgen von Entwicklung und Modernisierung - ein deutlicher Gegensatz zum zeitgenössischen Mainstream. Politische Stabilität lasse sich nicht anhand von Wachstumsraten oder ähnlicher Parameter voraussagen. Entscheidend für die politischen Folgen gesellschaftlichen Wandels, also für die Frage von Stabilität oder Instabilität, so Polk, sei vielmehr die politische Integration bzw. Exklusion neuer Schichten, der „New Men“, die er als diejenige Gruppe definierte, die die Ausbildung, Motivation und Orientierung besäße, um eine Gesellschaft zu modernisieren. Im revolutionären Ägypten gelänge dies gegenwärtig. So erklärte Polk die im Grunde ökonomisch zweifelhaften Industrialisierungsbemühungen der VAR mit dem politischen Ziel, sich selbst

\footnotetext{
${ }_{159}$ In der Zukunft wäre ein Drängen nach größerer Autonomie seitens der Regierungsbürokratie oder auch eine Parlamentarisierung Irans als Folge einer Inanspruchnahme der konstitutionellen Rechte durch den gegenwärtig noch gleichgeschalteten Mağles denkbar. Siehe Memo, ,Potential Policy Problems in Iran Through 1968 , 6.10.64, ,Office Memoranda', Lot 69 D 30, NEA/IRN: 1964-66, Box 1.

${ }^{160}$ Vgl. POLK, Analyzing Social Change; Polk Letter, 23.4.65, , Near and Middle East 1965-66', Lot 72 D 139, S/P Records, Subject and Country: 1965-69, Box 315; Polk Letter, 3.3.63, , Iran 1/63-11/63', NSF, RWK, Box 424, JFKL; eine Würdigung der Bedeutung Polks in JACOBS, Imagining the Middle East, S. 45f.; CiTINO, Envisioning the Arab Future, S. 212-50.
} 
eine gesellschaftliche Machtbasis zu schaffen und die ägyptischen „Neuen Männer“ mit dem eigenen Herrschaftsanspruch zu identifizieren. Diese neuen Gruppen - geformt durch Ausweitung der Bildung und dem Militär als Schule der Nation - seien die hauptsächlichen Profiteure der revolutionären Transformation in Ägypten. ${ }^{161}$ Anders sei die Entwicklung im Irak in den 1950ern zu erklären. Dort habe die Regierung ebenfalls aktive Entwicklungspolitik betrieben und eine Klasse von New Men generiert. Gleichzeitig aber habe die traditionelle Klasse die neuen Schichten aus jeder echten politischen Mitwirkung ausgeschlossen und politisch unterdrückt. Die Modernisierung der Sicherheitsapparate und insbesondere des Militärs wiederum habe diese Institutionen zu Bastionen der neuen, technologisch befähigten Schicht gemacht, was letztlich im Militärcoup von 1958 resultierte. Iran sei, so Polk, dasjenige Land, dessen Entwicklung der in Irak am ehesten gleiche. Die rapiden Modernisierungs- und Industrialisierungsanstrengungen des Schahs würden Umfang und Einfluss der neuen Schicht ausweiten - zugleich habe die „Revolution von Oben“ die traditionelle Schicht - ,the traditional element, with which royalty is generally associated“ - maßgeblich geschwächt. Im Grunde, so Polk implizit, sei der Schah dabei, sich selbst abzuschaffen. ${ }^{162}$

Diesbezüglich expliziter wurde Polk in internen Memoranden aus dem Jahr 1965. Hier erscheinen die New Men explizit als die „New Power Elite“, die es zu integrieren gelte, wolle man Stabilität in den sich modernisierenden Gesellschaften bewahren: „The central question posed by the modernization process is the means of effecting a new political synthesis in which the modernizers are included. “163 Hier findet sich in der Tat die „,new political synthesis“ des Task Force Report von 1961 wieder. Politische Transition in den Staaten des Nahen und Mittleren Ostens sei zwangsläufig. Die Frage sei nicht ob, sondern wann diese stattfinde. ${ }^{164}$ Das rasche Bevölkerungswachstum gehe mit dem noch rascheren Anwachsen politisch mobilisierbarer Mittelklassen einher. Hinzu käme der Trend zur rapiden Urbanisierung. An den Rändern der Städte wachse infolge der Landflucht, so Polk, ein neues Proletariat - ,a new layer of society for politics“ - heran. Gegenwärtig könne noch niemand diese Gruppen mobilisieren. In etwa 10 Jahren aber seien radikale proletarische Parteien denkbar und diese würden dann nicht vom Gedankengut der westlich-modernistisch orientierten Gemäßigten, sondern wahrscheinlich von kommunistischen Ideologien oder der Weltsicht religiösfundamentalistischer Gruppierungen wie der Muslimbruderschaft maßgeblich beeinflusst. „Post-revolutionäre“ Staaten wie die VAR, Irak oder Algerien hätten bessere Chancen, dieser

\footnotetext{
${ }^{161}$ Vgl. PoLK, Analysis, S. 17.

${ }^{162}$ Ebd., S. 18; bezüglich Irans (und Marokkos) schlussfolgerte Polk für die US-Politik: „Only if the United States is able to assist in the peaceful and gradual inclusion of new elements in the structure of power, will it find its position in both of these countries considerably augmented."; ebd., S. 19.

163 Polk Memo, ,A Look Ahead at the Middle East and North Africa', 1.2.65, ohne Folder, Lot 69 D 225 , NEA/IRN: 1964-66, Box 6, RG 59, NACP \{Hervorheb. i. Orig.\}; der Modernisierungsprozess sei kein Effekt des Kalten Krieges, würde aber durch die Systemkonkurrenz massiv beschleunigt. Kein Staat, nicht einmal der rückständige Nordjemen, könne sich diesem Sog entziehen. Polk fasste dies in einer kontra-intuitiven Aussage zusammen: "There is no regime which does not put a considerable part of its effort into creating the potential of revolution."

${ }^{164}$ Auch hier griff Polk wieder auf das historische Beispiel des Irak in den 1950er Jahren zurück.
} 
Herausforderung zu begegnen, da dort die ,neuen Eliten“ bereits die Macht oder wenigstens Teile davon innehätten. In den ,vor-revolutionären“ Staaten aber sei zuerst einmal die Herstellung eines friedlichen Kompromisses mit den „,modernizers“ vonnöten. ${ }^{165}$ Ansonsten drohe gewaltsamer politischer Wandel mit entsprechenden Nachteilen für amerikanische strategische Interessen: „The US is already closely identified with the existing regimes, the Soviet Union is not and in a process of violent change American interests are certain to be adversely affected."166

Iran aber war für Polk der, ,vor-revolutionäre“ Vorzeigestaat. Hier stünden sich das ,,politische Establishment" - Hof, Teile der Bürokratie und Armee - und ,wirtschaftliches Establishment“ - eben die neuen funktionalen Eliten - unversöhnlich und kompromisslos gegenüber. Die Verhandlungsposition des Schahs würde in der nahen Zukunft vermeintlich wachsen aufgrund gestiegener Öleinnahmen und verschärfter Repression. Zugleich aber wachse die Zahl der „,neuen Männer“ ebenfalls rasant an und diese würden zusehends die wirtschaftlichen und administrativen Schlüsselpositionen besetzen. Der Schah müsse letzteren ,political recognition of their economic and social power" gewähren, ansonsten werde Iran auf einen Konflikt oder eine Revolution zusteuern: „What the Shah does during this [...] period may determine Iran's history for generations. “167 Sollten die New Men von Regierungsbeteiligung und politischer Macht ausgeschlossen bleiben,

[...] they will be thrust aside and others will use more violent means. As violence increases, the Shah will appear, for a while, to be able to keep the situation under control by repression but sooner or later his security forces will do him in or will refuse to fire on those who will. ${ }^{168}$

Polk warnte explizit davor, die gegenwärtige Ruhe in Iran und die sich rasch verbessernde wirtschaftliche Lage überzubewerten. Der grundsätzliche Konflikt sei ungelöst und schwele weiter verborgen unter der Oberfläche. ${ }^{169}$

\footnotetext{
${ }^{165}$ Man dürfe sich nicht von der scheinbaren Schwäche der politischen Opposition in manchen dieser Staaten täuschen lassen: „It will be extraordinarily difficult both for these governments and for us to measure the potential for change as the opposition groups can seem pitifully small and politically insignificant any yet, if they occupy key places in the social and economic structure and can embody generally held sentiments, can perform near miracles. The pre-history of the Iraqi and Algerian revolutions bear witness to this fact."; ebd.; sehr wahrscheinlich, so Polk, würden die jeweiligen Regierungen die Stärke der Opposition falsch einschätzen und ,,take the short road to ruin by trying to suppress discontent".

${ }^{166}$ Ebd.

${ }^{167}$ Polk Memo, 26.10.64, ebd.

168 Polk Letter, 23.4.65, Lot 72 D 139, S/P Records, Subject and Country: 1965-69, Box 315.

${ }^{169}$ Wieder bemühte Polk das Beispiel des Iraks: ,The irony here [...] is that our tools of intelligence of analysis fail us at this point. You know Iraq of 1950-1958 well. You know there that the Iraqi equivalent of the National Front et al was weak, divided, ineffectual, gutless; the government was headed by a wise old pro and a man who was admitted, even by those who hated him, to be so popular that he could win an open election; the government was getting richer and stronger from oil revenues, building projects, better communications, a modernized army and US trained security forces. On the surface, the government strength was growing and the opposition sapped by prosperity and hope. Then came 1958. As a historian I can tell you that a similar situation existed before each of the major revolutions in the last two centuries."; ebd.
} 
Polks Warnungen hinsichtlich der Zukunft Irans aber wurden ignoriert. ${ }^{170} \mathrm{Zu}$ gefestigt war inzwischen der Konsens über die Erfolgsaussichten der Weißen Revolution Mohammad Rezās und über dessen Regierungsgeschick. Mitte der 1960er Jahre gab es auf amerikanischer Seite so gut wie keine Bereitschaft mehr, reformistisch-interventionistische Experimente in Iran zu wagen. Verglichen mit den Jahren zuvor schien Iran in der Tat auf dem Weg, ein Musterbeispiel für amerikanische Klientelstaaten an der Peripherie zu werden, zumindest in der - wesentliche Details der Entwicklung ausblendenden - historischen Rückschau. Hinzu kam die geänderte Weltlage und die Konzentration auf Südostasien und die massive militärische US-Intervention dort, die den Nahen und Mittleren Osten vorübergehend zu einem Nebenschauplatz werden ließ. Wichtig ist aber auch, dass Kritiker der offiziellen US-Politik wie Polk, Miller oder Hansen den amerikanischen Einfluss in Iran fortwährend überschätzten. Seit den frühen 1960er Jahren hatte Mohammad Rezā systematisch den amerikanischen Einfluss in Iran zurückgedrängt, um die eigene politische Macht und Handlungsfreiheit zu mehren und zu sichern. Mitte der Dekade hatte sich das bilaterale Verhältnis in der Tat grundlegend gewandelt.

\section{3. Souveränität und Gleichrangigkeit: Das Ende permanenter Intervention und die Transformation der Beziehungen}

Wenig erwünscht aus amerikanischer Sicht war sicherlich die Kehrseite der neuen Unabhängigkeit Irans nach innen wie außen. Bereits im Kontext der mehrjährigen Auseinandersetzungen über die Militärkredite und das amerikanische Rüstungsmonopol hatte sich ein zunehmend anti-amerikanischer Ton in Iran bemerkbar gemacht. Die innere Opposition, die sich allerdings kaum noch öffentlich in Szene setzten konnte, hatte nach 1963 die Hoffnung auf eine mögliche Unterstützung der USA für eine Machtbeteiligung aufgegeben. Die immer prägnanter werdende öffentliche Unterstützung der Kennedy- und JohnsonAdministrationen für die Schah-Diktatur und das Reformprogramm hatten der Amerikafeindlichkeit neuen Auftrieb gegeben, insbesondere unter der weitaus radikaleren neuen Generation der Nationalisten. Das frühere Image der USA als demokratische Vorzeigenation und damit eigentlich natürlicher Verbündeter der liberal und national gesinnten Opposition wich dem einer imperialistischen und expansiven Macht, ironischerweise eben zu dem Zeitpunkt, als sich der politische Einfluss der USA stark zurückentwickelt hatte. Noch stärker anti-amerikanisch gebärdete sich die religiöse Opposition. Mit dem Beginn des Vietnam-Kriegs steigerte sich auch in Iran die anti-imperialistische und anti-amerikanische Rhetorik - selbst die staatlich kontrollierte Presse erging sich in „America bashing“, was im

\footnotetext{
${ }^{170}$ Ein Brief GTIs nannte Polk den ,most intellectually respectable of the critics of our policy towards Iran. He has heard our arguments over and over and remains rather firmly aligned with the Bill Miller position."; Howison Letter, 17.3.66, ,POL 7 Visits. Missions. 1966‘, Lot 70 D 330, NEA/IRN: 1964-66, Box 18.
} 
Herbst 1966 sogar die US-Botschaft bewog, direkt beim Schah gegen die Berichterstattung zu intervenieren. ${ }^{171}$ Tatsächlich spricht einiges dafür, dass die Berichterstattung, und auch die Veröffentlichung Amerika-kritischer Bücher in dieser Zeit trotz der ansonsten effizienten Zensur, ganz bewusst vom Hof toleriert wurde, wohl auch im Sinne der neuen „Unabhängigen Nationalen Politik“. ${ }^{172}$ In der Tat war das zuvor durchaus feststellbare Vertrauensverhältnis einer distanzierteren Beziehung gewichen. So zeigte der SAWAK nun auch merkliches Interesse an amerikanischen Aktivitäten in Iran und warnte iranische Bürger vor engeren Kontakten mit US-Offiziellen. ${ }^{173}$

Spätestens ab 1965 war augenfällig, dass sich das ehemals stark ungleiche Verhältnis zwischen den USA und Iran grundsätzlich geändert hatte. Die auch öffentlich ausgetragenen Konflikte über die für das bilaterale Verhältnis zentralen Militärbeziehungen und Waffenlieferungen waren letzten Endes ein Ausdruck des sich verschiebenden Machtgefüges im beiderseitigen Verhältnis. Mit dem Scheitern des reformistischen Interventionismus der Vorjahre und insbesondere der Erlangung finanzieller Autonomie infolge der rapide gestiegenen Öleinnahmen war der vormals präponderante amerikanische Einfluss stark zurückgewichen. Iranische Souveränität wurde in allen Politikfeldern nun mit Nachdruck und kompromisslos durchgesetzt. Die Einbeziehung der Öffentlichkeit während der Auseinandersetzungen über das Militärprogramm, die zunehmend Amerika-kritische Einstellung der von der Regierung gesteuerten Presse und die Distanzierung vom bisherigen Patron in einer Reihe von symbolischen Akten, häufig im Rahmen der hektischen Reisetätigkeit des Schahs, dienten allesamt der vollständigen Durchsetzung der vollen iranischen Unabhängigkeit und Souveränität. „They are no longer willing to 'be bossed'“, so die Warnung aus der Teheraner Botschaft - der letztendliche Ausgang der Streitigkeiten um das Aufrüstungsprogramm und das vollständige amerikanische Einlenken 1966 illustrierten die Richtigkeit dieser Aussage. ${ }^{174}$ Während der internen Beratungen über die Militärhilfe an Iran hatte der Nachfolger Talbots als Assistant Secretary of State for Near Eastern and South Asian Affairs, Raymond Hare, explizit festgestellt, ,[...] we are in a new phase; we have seen the end of the 'client relationship' ."175

\footnotetext{
${ }^{171}$ Vgl. Tehran \#A-235, 29.10.66, POL IRAN-US; Tehran \#A-164, 22.8.66, POL 2-3 IRAN, CF 64-66.

172 Vgl. BILl, Eagle and Lion, S. 1178f.; der Schah gestand dies sogar indirekt ein: „He added that any unfriendliness of Iranian opinion is at least part due to America's past mistakes. He cited what he considers virtually public USG \{U.S. government; R.P.\} support for Amini as ,last chance for Iran,'[...]“; Tehran \#1762, 29.6.66, FRUS 64-68, XXII, S. 265.

${ }^{173}$ Vgl. Meshed \#A-12, 2.12.65, POL IRAN-US, CF 64-66, RG 59, NACP; auch werde nunmehr häufig die an Amerikaner in Iran gerichtete Post geöffnet. Zur Aufrechterhaltung der Kontakte zur Opposition vgl. unten S. $528 \mathrm{f}$.

${ }^{174}$ Tatsächlich stammte die Bemerkung von einem israelischen Diplomaten. Meyer Letter, 19.3.66, ,Iran', Lot 68 D 117, Subject Files 1965-66, ASS/NEA, Box 2; in demselben Brief hatte Meyer vor Versuchen gewarnt, das alte Verhältnis fortzuführen. Die Annual Review über die Balance des Rüstungs- mit dem Entwicklungsprogramm ,will undoubtedly become more and more a symbol to the Shah of the old relationship which the Shah increasingly detests, whereby we 'dictated' to him what was best for Iran."; ohne ein Einlenken und - mittelfristig - der Aufgabe der Annual Review, so Meyer, drohe eine öffentliche „Unabhängigkeitserklärung“ Irans, die einseitige Kündigung der MAP-Vereinbarung von 1962 und des MOUs von 1964 und wohl das Ende von ARMISH/MAAG.

${ }^{175}$ Near East-South Asia IRG Meeting Minutes, 12.5.66, FRUS 64-68. XXII, S. 243 \{Hervorheb. v. Verf.\}; analog wurden die persönlichen Beziehungen zwischen dem Monarchen und dem jeweiligen US-Präsidenten umso wichtiger. So z.B. Komer mit dem ihm eigenen Hang zur Übertreibung: „Now for the President of the United
} 
Das Ende der Patron-Klienten-Beziehung ergab sich sowohl aus dem oben beschriebenen strukturellen Wandel als auch aus der persönlichen Entschlossenheit iranischer Offizieller, aber vor allem des Schahs, die als belastend und einschränkend empfundene Beziehung zu Washington zu revidieren. Mohammad Rezā selbst sah den Zeitpunkt für eine solche Neujustierung gekommen, zum einen, da er durch die Entwicklung der vergangenen Jahre enorm an Selbstbewusstsein und Handlungsautonomie gewonnen hatte, zum anderen, da die Erinnerung der Jahre seit 1958 und die von ihm so wahrgenommene Infragestellung seiner Befähigung als Herrscher durch die Schutzmacht im Kontext der reformistischen Intervention eine zusätzliche Motivation für die Beseitigung des amerikanischen Einflusses darstellte. Das gewachsene Selbstbewusstsein im Vergleich zu 1962 war vier Jahre später kaum zu übersehen:

\begin{abstract}
Never in his twenty-five year reign has the Shah been more firmly in the saddle. The Shah's current attitudes are in a sense attributable to successful United States policy which has aimed steadily over the years to make Iran more self-reliant. In addition there are sound reasons for this enhancement of his position. Iran's economy is thriving. No LDC \{less developed countries; R.P.\} can match the progress which Iran is making in the economic and social fields [...]. The steel-mill-for-gas-deal with the Soviet Union has taken some of the wind out of the sails of his nationalist opposition and has created optimistic expectations among the populace at large. The growing friendliness of the USSR towards Iran has created dispiritedness among the leftist (Tudeh) forces. Both nationalist and leftist elements, perennially eager for power, are without flags to wave. Many former nationalists and Tudeh party types have thrown in the sponge and, at least for present, are applauding Iran's progress. Last but not least, Iran's oil income is soaring to new heights giving assurance that Iran's new, independent policy has sound underpinnings. ${ }^{176}$
\end{abstract}

Amerikanische Offizielle vor Ort waren sich aber zugleich durchgehend bewusst, dass die teilweise scharfen Reaktionen Mohammad Rezās auch auf die von ihm so empfundenen Demütigungen der vergangenen Jahre und seine Interpretation der möglichen Folgen einer allzu engen Abhängigkeit von der Supermacht zurückzuführen waren: „The level of anxiety and distrust of the U.S. is still influenced by his understanding of the U.S. policy produced by the Iran Task Force in May 1961 and the destruction of the Diem regime in Vietnam in 1963.“ Beide Ereignisse seien inzwischen „Teil der Geschichte“, doch würden sie prägend auf die Attitüde des Schahs gegenüber den Vereinigten Staaten einwirken. ${ }^{177}$

Offenbar empfand der Schah den früheren Einfluss der USA in Iran ex post facto als so einschneidend und mit seiner monarchischen Würde unvereinbar, dass er die Geschichte der amerikanischen Involvierung in seinem Land im Nachhinein zu revidieren trachtete. In einer für die Gesamtheit der amerikanisch-iranischen Beziehungen erhellenden Episode stellte der Schah im Sommer 1965 die frühe Involvierung der USA in Iran im Kontext des aufziehenden

States, the Shah will push a peanut with his nose if it's necessary to get along well with him. "; Robert W. Komer OHI, 30.1.70, LBJL, S. 56.

176 Tehran \#A-738, 7.5.66, DEF 19 US-IRAN, CF 64-66.

177 CIA Memo, encl. to Critchfield Memo, 22.3.66, ,IRG/NEA Miscellaneous Material', Lot 68 D 117, Subject Files 1965-66, ASS/NEA, Box 5. 
Kalten Kriegs in Frage, ein Ereignis, das bislang immer als vorbildlicher Einsatz einer desinteressierten Macht zur Verteidigung der Unabhängigkeit Irans gegenüber der UdSSR (und indirekt gegenüber Großbritannien) interpretiert worden war. Der Schah glaubte sich nun, zwanzig Jahre später, zu erinnern, der damalige Botschafter Wallace Murray habe ihn damals über eine Absprache zwischen den drei Hauptmächten der Anti-Hitler-Allianz informiert, derzufolge eine Form von Autonomie in Kurdistan, Aserbaidschan und Xuzestān einzurichten sei - sogar eine Aufteilung des Landes zwischen den drei Mächten sei im Gespräch gewesen. Zudem habe George Allen während der Aserbaidschankrise explizit jede militärische Unterstützung Irans ausgeschlossen. ${ }^{178}$ NEA bemühte sich daraufhin in Zusammenarbeit mit ehemaligen Offiziellen und dem Historical Office im State Department um die Korrektur dieser Behauptungen: Tatsächlich habe vorwiegend amerikanischer Druck erst den Abzug der Sowjets und die Wiederherstellung der iranischen Souveränität und Integrität ermöglicht amerikanische Rückendeckung sei zudem entscheidend gewesen für den Erfolg der Militäraktion gegen Täbris Ende 1946. ${ }^{179}$ Das (bewusst oder unbewusst) selektive historische Erinnerungsvermögen Mohammad Rezās ist dabei weniger wichtig. Interessant ist vielmehr, dass der Schah nun ein historisches Schlüsselereignis, den historischen Ausgangspunkt amerikanischer Involvierung in die inneren Angelegenheiten Irans, im Zuge seiner aktuellen Auseinandersetzungen mit der US-Diplomatie umdeutete: Er stellte die USA nunmehr nicht mehr als Retter Irans sondern vielmehr als eine von dreien im Grunde auf die Kontrolle Irans abzielenden Mächte dar. In der Folgezeit setzten sich derartige Vorwürfe fort. So habe die USRegierung , ,...] sought to dictate Iran's policies from days of Gerry Dooher (who 'wanted to be Lawrence of Iran") to Amini days of early sixties."180 Man kann in diesen „Geschichtskontroversen“ einen frühen Ausdruck der später für die Dekaden des Antagonismus zwischen beiden Staaten so prägenden Auseinandersetzungen über bestimmte historische Ereignisse sehen, die bis heute eine Annäherung zwischen beiden Ländern erschweren.

Der im Jahr 1964 46-jährige Monarch wurde seit Einleitung des umfassenden Reformprogramms von amerikanischer Seite grundsätzlich positiv eingeschätzt. Nichts mehr zu finden war von den früheren Einschätzungen, wonach Mohammad Rezā Teil des Problems und nicht die Lösung für Iran war. Im Gegenteil: die CIA schrieb ihm „,some considerable skill““ hinsichtlich seines Managements in der jüngeren Krisenphase und der Durchsetzung seiner persönlichen Herrschaft zu. Schon immer hatte man ihm eine überdurchschnittliche Intelligenz und einen umgänglichen Charakter sowie ein genuines Interesse am Fortschritt in Iran zugesprochen - nun aber schien er auch die Herrschaftsmethoden verinnerlicht zu haben, um

\footnotetext{
${ }^{178}$ Vgl. Tehran \#244, 31.8.65, FRUS 64-68, XXII, S. 168ff.

179 Vgl. Herz Letter, 18.10.65; Franklin Letter, 29.10.65, POL IRAN-US; Geheimdienstveteran Edwin Wright schilderte ein weiteres Mal die genauen Umstände der Konfrontation in der Aserbaidschankrise vom Frühling 1946 und des sogenannten ,Truman-Ultimatums“. Vgl Wright Memo, 16.8.65, POL 23-7 IRAN, CF 64-66; tatsächlich hatte sich der Schah bereits 1960 einmal über die genauen Vorgänge erkundigt. Vgl. Tehran \#SECTO10, 28.4.60, CF 60-63, 688.00/4-2860; Mouser Memo, 10.5.60; Allen Memo for the Files, 12.5.60, ,16 USSR-Iran Relations 1960‘, NEA, GTI: RIAD, 1958-63, Box 2; siehe auch oben, Kapitel II.

${ }^{180}$ Tehran \#776, 25.11.65, FRUS 64-68, XXII, S. 189.
} 
einen solchen erfolgreich $\mathrm{zu}$ bewerkstelligen. ${ }^{181}$ Die Botschaft konstatierte in einem Psychogramm des Monarchen, Mohammad Rezā ähnele zunehmend seinem Vater. Sein jugendlich-schwankendes Gemüt sei einer neuen Sicherheit gewichen - „he is more firmly in saddle in Iran than ever before." Aufgrund der historischen Erfahrungen Irans, sei sein Hauptinteresse neben der materiellen und gesellschaftlichen Stärkung seines Landes (,Shah has almost pathological sense of mission for his country") die Durchsetzung voller Unabhängigkeit und Handlungsfreiheit. Daraus erkläre sich sein Bemühen, Anerkennung im „unprincipled Afro-Asian political milieu“ zu finden, obgleich seine inneren Überzeugungen mit denen des Westens kongruierten. Seine großen Schwächen aber seien unverändert: eine Obsession mit allen militärischen Dingen und seine Unfähigkeit mit Kritik umzugehen. Das zeitweilig als ungenügend empfundene Entgegenkommen Washingtons im militärischen Bereich, die amerikanische Ablehnung der Ernsthaftigkeit der „,arabischen Gefahr“ und das ungehinderte Operieren der Auslandsopposition in den USA zusammen mit kritischer Berichterstattung amerikanischer Medien über sein Regime verstärkten sein Misstrauen gegenüber den USA. ${ }^{182}$

Zugleich machte sich das wachsende Selbstvertrauen ab Mitte der 1960er Jahre immer stärker bemerkbar. Gegenüber Averell Harriman konkretisierte Mohammad Rezā im November 1966 seine Zukunftspläne für Iran. So gab er als Zielsetzung aus, innerhalb der nächsten 20 Jahre zum Entwicklungsstand der europäischen Länder aufschließen zu wollen. Außer Iran könne dies nur Japan erreichen, so der Schah in einer in der Zukunft fast gebetsmühlenartig wiederholten Formulierung. Eng verbunden mit den ambitionierten wirtschaftlichen Zielsetzungen war die Projektion der zukünftigen sicherheitspolitischen Rolle Irans. Mit dem für 1968 angekündigten britischen Abzug aus Südarabien und Aden ergäbe sich ein Machtvakuum. Der Schah sah die zukünftige Aufgabe Irans in der Aufrechterhaltung regionaler Ordnung und der Wahrung des Friedens - Voraussetzung für die Ausübung einer solchen Rolle waren selbstverständlich der Aufbau entsprechender Kapazitäten zur eigenen militärischen Machtprojektion: „Shah felt Iran's capability for handling regional disturbance is as much in USG's \{U.S. Government's; R.P.\} interest as that of Iran.“"183

Angesichts der inzwischen kaum noch ernsthaft infrage gestellten Dominanz des politischen Feldes in Iran im Zusammenspiel mit der Ablösung des Klientelstatus gegenüber den USA und

\footnotetext{
${ }^{181}$ CIA Memo, OCI No. 1582/64, 30.5.64, FRUS 64-68, XXII, S. 62; neben Sport habe er Interessen an Kunst und Literatur, , as well as in attractive women', ein Hinweis auf die zahlreichen außerehelichen Affären. Vernichtend dagegen war das Urteil über die kaiserliche Familie und Entourage. Bezüglich letzterer urteilte die CIA: „The political philosophy of many of the Shah's court intimates appears to be late eighteenth century Bourbon."; auch seine Familie „has not been among his assets.“; seine Halbbrüder seien nur von begrenztem Nutzen und seine Zwillingsschwester Ašraf ,leads an uninhibited personal life allegedly engaging in such doubtful business enterprises as opium smuggling [...].“; ebd., S. 61; Mohammad Rezā schien in der Tat anfällig für Speichellecker. Vgl. AzIMI, Quest for Democracy, S. 230f.; eine interessante Einschätzung des Charakters des Monarchen bei AMUZEGAR, Dynamics, S.213-21; stark psychologisierend Zonis, Majestic Failure, S. 2f., 7-22, der Mohammad Rezā eine narzisstische und größenwahnsinnige Persönlichkeit attestiert. Mit den Quellen m.E. nicht vereinbar die Interpretation von OFFILER, Metaphor and Gender.

${ }_{182}$ Vgl. Tehran \#789, 28.11.65, ebd., S. 195-200, Zitate auf 195 und 196 sowie in Tehran \#1222, 9.3.66, ,Iran Cables, Vol. 2 [2 of 2]', NSF, Country File, Box 136, LBJL; vgl. CIA Memo, 30.3.66, FRUS 64-68, XXII, S. $227 f$. ${ }^{183}$ Tehran \#1964, 2.11.66, ebd., S. $328 f$.
} 
den außenpolitischen Erfolgen zeichnete sich Mitte der Dekade zudem eine weitere Steigerung der Selbstherrlichkeit Mohammad Rezās ab. Anflüge von Hybris waren unübersehbar, aber im Vergleich zu den späten Jahren noch moderat. Zum 25-jährigen Thronjubiläum 1965 wurden „überwältigende“ Festlichkeiten organisiert - zugleich ließ sich der Schah vom Mağles den Ehrentitel Arjāmehr - „Licht der Arier“ - verleihen. ${ }^{184}$ Dies war der Startpunkt für die nun einsetzende ausufernde und im Ausland häufig gleichermaßen belächelte wie bewunderte Außendarstellung der Pahlawi-Dynastie, die sich - wohl nicht zuletzt im Bewusstsein der eigenen, doch alles andere als noblen Herkunft - in Gepränge, Prunk und Pomp selbst überbot. ${ }^{185}$ Tatsächlich war dieser unabhängige wie selbstbewusste Schah noch weniger willens, Erklärungsversuche der US-Administration über die in einer Demokratie bestehenden Handlungsbeschränkungen gegenüber der Ausübung von Grundrechten wie Meinungs- und Versammlungsfreiheit zu akzeptieren. Aus den eigentlich eher peripheren Aktivitäten iranischer Studenten in den USA und der sogenannten Gudarzijān-Affäre konnten so erhebliche Belastungen des bilateralen Verhältnisses erwachsen. Die „Veröffentlichung“ der konflikthaften Zuspitzung der Krise in den bilateralen Beziehungen in 1966 durch den Schah in einer Rede vom März vor dem Mağles erklärte Mohammad Rezā explizit, dass man keine „Befehle entgegennehme“, eine unmissverständliche Warnung an den amerikanischen Verbündeten - erklärt sich somit auch aus dem Bedürfnis, die bisherige Vormundschaft ostentativ abzulegen. ${ }^{186}$

Eine lang anhaltende Belastung des bilateralen Verhältnisses ergab sich aufgrund der sich aus der Gudarzijān-Affäre ergebenden Schwierigkeiten. Im Mai 1963 wurden Anschuldigungen eines in den Vereinigten Staaten lebenden Exiliraners namens Xajbar Xān Gudarzijān gegen die kaiserliche Familie bekannt. Gudarzijān, den US-Behörden als Trickbetrüger und Hochstapler bekannt - unter anderem gab er sich als Baxtijāri-Khan aus -, legte einem Subcommittee des US-Senats vermeintliche Beweise für Korruption der Familie Mohammad Rezās im Zusammenhang mit amerikanischer Finanzhilfe für Iran vor. ${ }^{187}$ Die Beweise waren

\footnotetext{
184 Vgl. Tehran \#A-306, 30.10.65, POL 15-1 IRAN, CF 64-66; auf die Mağles-Zeremonie und die offizielle Titelverleihung folgte am Abend des 15. September eine Zeremonie oberhalb Teherans mit dem Rezitieren von Lobpreisungsgedichten für frühere iranische Herrscher, gemeinsamem Gebet, Nationalhymne, Kanonensalut und einem aufwändigen Feuerwerk. Zudem wurden beleuchtete Nachbildungen der Pahlawi-Krone und der Schriftzug „Schah" im an Teheran angrenzenden Gebirgszug installiert. Am Tag danach folgten Empfänge und Paraden. Die Botschaft sprach von ,enormous expenditures“.

${ }^{185}$ Der Ehefrau Pākrawāns zufolge wurde erst Ende der 1950er ein striktes Hofprotokoll eingeführt, das die vorhergehende Ungezwungenheit abstellte. Offenbar hatte man sich vom britischen Hof in dieser Frage beraten lassen. Vgl. PAKRAVAN, Memoirs, S. 89f.

${ }_{186}$ Zur Rede und der US-Reaktion vgl. Tehran \#1185, 2.3.66, FRUS 64-68, XXII, S. 216f.; zu den verschiedenen Kritikpunkten des Schahs an der US-Politik in diesem Zeitraum vgl. Memo, The Shah's Personal Outlook', 15.7.66, encl. to Eliot Memo, 15.7.66, ,POL 15-1 Iran. Head of State 1966', Lot 70 D 330, NEA/IRN: 1964-66, Box 17.

${ }^{187}$ Gudarzijān hatte seit 1961 versucht, die iranische Regierung finanziell zu erpressen mit der Drohung, andernfalls die Affäre publik zu machen. Das Senate Permanent Subcommittee on Investigations, nach dem Vorsitzenden häufig McClellan Committee genannt, stimmte schließlich im Mai 1963 einer Anhörung Gudarzijāns zu. Dort legte er seine Beweise, gefälschte Schecks der Pahlavi Foundation an eine Reihe amerikanischer Politiker und ehemalige US-Botschafter in Iran, vor. Vgl. State \#838, 17.5.63, ,Iran General 5/63‘, NSF, Countries, Box 116A, JFKL; auf Betreiben Gudarzijāns forderte das Subcommittee von der iranischen Botschaft die Herausgabe der Finanzakten der Pahlavi Foundation. Rusk intervenierte umgehend und erfolglos bei Senator McClellan. Vgl. Grant Memos, 29.5.63/6.6.63, POL IRAN-US, CF 63.
} 
gefälscht. Angesichts weit verbreiteter Gerüchte über das Finanzgebaren innerhalb der kaiserlichen Familie und im Kontext der Bemühungen des Schahs, Fortschritte im Kampf gegen die Korruption vorzuweisen, erhielt die Affäre dennoch große mediale Aufmerksamkeit. Gudarzijān benutzte diese, um die Schah-Familie weiter unter Druck zu setzen. Er verklagte den jüngeren Bruder des Schahs, Prinz Mahmud Rezā, auf Basis eines gefälschten Schecks und erwirkte im Oktober 1964 ein Versäumnisurteil eines New Yorker Gerichts gegen diesen. ${ }^{188}$ Mit einiger Verspätung bemühten sich die US-Behörden nun um die Einleitung eines Strafverfahrens gegen Gudarzijān und leiteten zudem ein Ausweisungsverfahren an - zum amerikanischen Bedauern entwickelte sich die Affäre aber zu einem langjährigen Ringen vor den Gerichten. Mohammad Rezā zeigte keinerlei Verständnis für die diversen rechtlichen Handlungsbeschränkungen für die US-Behörden und empfand die fortwährende Nennung angeblicher illegaler Aktivitäten seiner Geschwister als öffentliche Brüskierung seiner Stellung und des iranischen Staates. Immer wieder kam es in der Folgezeit zu diplomatischen Verwerfungen infolge der Krise - der Schah ersetzte aus Verärgerung über die vielen Verzögerungen sogar den Botschafter in Washington. ${ }^{189}$ Obgleich der Vorsitzende des SenatsSubcommittee im April 1965 öffentlich die Unhaltbarkeit der erhobenen Vorwürfe erklärte, konnten die anhängigen Gerichtsverfahren nicht zeitnah abgeschlossen werden. ${ }^{190}$ Eine erneute Veröffentlichung des Journals The Nation verschaffte den rufschädigenden Vorwürfen weite Verbreitung. ${ }^{191}$ Eine Verurteilung Gudarzijāns und damit eine Beendigung der Affäre war nur mit Zeugenaussagen iranischer Akteure möglich - der zentrale Zeuge aber war der ehemalige Leiter der iranischen Planorganisation Abu 1-Hasan Ebtehāğ. ${ }^{192}$ Seit seiner mutwilligen Verhaftung und Inhaftierung über die Jahreswende 1961/62 hatte Ebtehāğ mit dem Schah endgültig gebrochen. Er war daher nicht bereit, zugunsten der kaiserlichen Familie auszusagen, obgleich eine Reihe von amerikanischen Freunden wie z.B. John McCloy und selbst Secretary of State Rusk ihn zu überzeugen suchten. ${ }^{193}$ Die sich endlos hinziehende Affäre und die

\footnotetext{
${ }^{188}$ Vgl. Tehran \#515, 5.11.64, FRUS 64-68, XXII, S. $111 \mathrm{ff}$; Memo to RAH, o.D., encl. to Senate Subcommittee Findings, 24.7.64, ,Alleged Corruption in United States in Iran', ,Iran', Lot 68 D 117, Subject Files 1965-66, ASS/NEA, Box 2; Gudarzijān wird als glaubwürdig eingestuft in der uninformierten Darstellung bei HULBERT, Interlock, S. 47-69.

${ }^{189}$ Vgl. Talbot Memo, 19.12.64, FRUS 64-68, XXII, S. 117ff.; Rusk signalisierte Ende des Jahres gegenüber Ārām umfassende Aktivitäten, um Gudarzijān das Handwerk zu legen. Bereits im Dezember nahm eine Federal Grand Jury Ermittlungen gegen Gudarzijān auf. Vgl. Read Memo, 18.2.65, ebd., S. 129f.; ebd., S. 130 n2; Department of Justice Letter to Rusk, 28.12.64, ,CO 123 IRAN“, WHCF, Confidential File, Box 9 [1 of 2], LBJL; Forugi wurde im Februar 1965 abberufen.

${ }^{190}$ Vgl. Wehmeyer Memo, 25.3.65, FRUS 64-68, XXII, S. 132; Tehran \#1351, 2.6.65, ,Iran, Vol. I, Cables 1/64$12 / 65^{\circ}$, NSF, Country File, Box 136, LBJL.

${ }^{191}$ Fred J. Cook, ,The Billion-Dollar Mystery“, The Nation, 12.4.65, S. 380-97.

192 Vgl. State \#1166, 24.6.65, FRUS 64-68, XXII, S. 164f.; State \#731/\#197, 4.5./26.8.65, POL 15-1 IRAN, CF 64-66; MemCon, 25.9.65, ,Iran - Gudarzian Case 1965', NSF, FRWK, Box 28; George Ball zufolge war das Hauptmotiv für die Weigerung Ebtehāğgs seine frühere Inhaftierung. Vgl. TelCons, 27.9./4.10.65, ,Iran [2/14/64/26/66]', Papers of George Ball, Box 4, LBJL; die Anklage gegen Ebtehāğ war im Februar 1964 fallen gelassen worden. Vgl. Tehran \#A-448, 19.2.64, POL 6 IRAN, CF 64-66; 11; tatsächlich war Ebtehāğ selbst nicht über jeden Verdacht der Vorteilsnahme erhaben. So hatte er im Frühjahr 1963 mit einer eher zweifelhaften Kampagne über den Einfluss ausländischer Banken in Iran das Ölkonsortium genötigt, einen Teil seines Pensionsfonds in Ebtehāğs eigener Bank zu deponieren. Vgl. Tehran \#A-581, 18.3.63, FN 6 IRAN, CF 63; im Gespräch mit George Ball wiederholte Ebtehāğ seine Sichtweise des Schah-Regimes als ,tyrannical, inefficient, corrupt and dictatorial [...] Disaffection from the Shah and his regime is intensifying, and an explosion is inevitable."; MemCon, 25.9.65, ,Gudarzian Case‘, Lot 74 D 272, Records of Under Secretary of State George W. Ball, 1961-1966, Box 26.

${ }^{193}$ Vgl. Tehran \#1117/\#63, 12.4./27.7.65, POL 15-1 IRAN, CF 64-66.
} 
öffentliche Desavouierung der kaiserlichen Familie stärkten somit den Verdacht Mohammad Rezās, die Administration zögere absichtlich eine rasche Beilegung hinaus, womöglich als Antwort auf die pro-sowjetischen Gesten des Schahs in jüngster Zeit und den Stahlhütten-Deal im Besonderen. ${ }^{194}$ Die Gudarzijān-Affäre zog sich weiter ungelöst hin. Die US-Behörden gaben 1967 die Strafverfolgung Gudarzijāns schließlich auf, da es ohne Aussage Ebtehāğs wenig Hoffnung auf Erfolg gab. ${ }^{195}$

Die bereits unter der Kennedy-Administration wenig ausgeprägte Bereitschaft, die Aktivitäten der iranischen Opposition in den USA und insbesondere die Umtriebe iranischer Studenten zu tolerieren, ließ unter der Johnson-Administration noch weiter nach. Während des Kurzbesuchs Mohammad Rezās in den USA im Juni 1964 fanden Protestaktionen der Studenten statt, in Umfang und Schärfe gesteigert im Vergleich mit dem letzten Besuch von 1962. Der Schah zeigte sich sehr irritiert über den Zwischenfall und bezeichnete die Demonstration gegenüber US-Offiziellen als Teil einer internationalen Verschwörung Nassers und der UdSSR gegen seine Person. ${ }^{196}$ Auf Betreiben des State Departement wurden einige oppositionelle Studenten im Mai 1964 vom Immigration and Naturalization Service zu Gesprächen einbestellt. Dort erhielten sie eine Warnung bezüglich ihrer politischen Aktivitäten - zwar seien friedliche und „nicht beleidigende“ Demonstrationen gegen den Schah weiterhin gestattet. Bei Nichtbefolgen und bei Vernachlässigung des Studiums drohten aber Deportation. Die US-Behörden versuchten zudem weiterhin, sich unangenehmer Oppositioneller wie z.B. dem zukünftigen post-revolutionären Außenminister Sādeg Ġotbzādeh zu entledigen. ${ }^{197}$ Die meisten der etwa

194 So hatten die Verfahren in den USA die Iraner bereits $\$ 100.000$ gekostet. Vgl. Tehran \#355/\#743, 13.9./18.11.65, FRUS 64-68, XXII, S. 175-9, 187f.; vgl. auch LADJEVARDI, Xāterāt-e Forugi, S. 145-50.

195 Gudarzijān hatte im Januar 1966 die Einleitung eines neuen Verfahrens erreicht, was zu weiteren Verzögerungen führte. Vgl. Kearney Memo, 28.10.65, POL 15-1 IRAN, CF 64-66; im April 1967 wurde die Botschaft schließlich informiert, ohne Aussage Ebtehāğs sei eine Verurteilung Gudarzijāns kaum zu erwarten. Alternativ bemühte man sich nun um die Deportation Gudarzijāns. Vgl. ed. note, FRUS 64-68, XXII, S. 354; die Affäre wirkte auch in späteren Jahren nach. Vgl. State \#78854, 5.5.72, FRUS 69-76, E-4, doc. 186.

${ }^{196}$ Insbesondere ein Flugzeug mit einem Transparent mit der Aufschrift ,If you want a fix, see the Shah“, offenbar eine Involvierung Mohammad Rezās in den globalen Heroinhandel suggerierend, erregte den Ärger des Schahs. Das State Department besprach die Sicherheitsfragen fortwährend mit SAWAK-Offizieren in der iranischen Botschaft. 1968 bat der Schah die ihm nahestehende CIA, für zusätzlichen Schutz während seiner USA-Besuche zu sorgen, da er der Kompetenz des State Department misstraute. Vgl. State \#834/\#931, 12.5./16.6.64, FRUS 6468, XXII, S. 36f., 90f.; die Polizeibehörden arbeiteten in der Folgezeit eng mit pro-monarchischen Studentengruppen zusammen, die auch als Hauptinformanten über die Studentenszene dienten, um in der Zukunft ähnliche Zwischenfälle zu vermeiden. Vgl. L.A. Division of Investigations Memo, ,Iranian Students', 6.7.66, ,POL 13-2 [...]1966', Lot 70 D 330, NEA/IRN: 1964-66, Box 17; CIA DD/S Diary Notes, 31.5.68, CREST: \#RDP73*001-3; die iranische Führung versuchte zudem, eine eigene pro-monarchische Gruppierung unter den Studenten aufzubauen. Vgl. FBI Field Report, The Iran House in Metropolitan Washington', 17.4.64, FOIA N ${ }^{\circ}$ 1201287-000 des Verfassers \{deklassifiziert am 22.1.2013\}; der Vorwurf der Studenten über die Involvierung von Teilen der kaiserlichen Familie in den Opium- und Heroinhandel war offenbar nicht unbegründet. Die Botschaft hatte einen dem dominikanischen Diktator Trujillo nahestehenden Währungsbetrüger und Heroinschmuggler identifiziert, der sich im Umfeld Ašrafs aufhielt. Vgl. Santo Domingo Operations Memo, 6.10.65, ,POL 6 -Leland Rosemberg 1966“, Lot 70 D 330, NEA/IRN: 1964-66, Box 18; vgl. GingerAS, Poppy Politics, S. $326 \mathrm{f}$.

${ }^{197}$ Ġtbzādeh wurde nach einer Reise nach Großbritannien im Anschluss ein US-Visum verweigert, da er offenbar versucht hatte, das Ablaufdatum seines Reisepasses zu manipulieren. Das State Department informierte die iranische Botschaft sogleich über diesen „Erfolg“. Dem früheren iranischen Innenminister "Ali Šājegān drohte ebenfalls die Ausweisung, doch wurde die weiterhin vom Justizdepartment hinausgezögert. Vgl. Talbot Memo, 13.5.64, FRUS 64-68, XXII, S. 37ff.; Tiger Letter, 29.4.64; Jernegan Memo, 28.5.64 ,POL 13-2 Students IRAN 1964', Lot 69 D 225, NEA/IRN: 1964-66, Box 6; Rusk Letter, 11.10.63; R. Kennedy Letter, 16.10.63, ,V 21 Fatemi, Ali M.S. 1965-66', Lot 69 D 491, NEA/IRN: 1964-66, Box 13; allerdings bemühte man sich seitens der Botschaft auch um Garantien seitens der iranischen Regierung, dass deportierte Studenten nach ihrer Rückkehr nicht behelligt würden. Vgl. Herz Letter, 4.10.65, ,POL 13-2-b [...]65', Lot 69 D 489, NEA/IRN: 1964-66, Box 
5-6.000 Studenten in den USA entstammten allerdings der iranischen Oberschicht und nur eine Minderheit war politisch engagiert. ${ }^{198}$ Die CIA bestätigte, einige dieser Studentenorganisationen würden von sowjetischer Seite heimlich finanziert, doch war man sich der Eigenständigkeit der Protestbewegung durchaus bewusst - das Dilemma zwischen bürgerlichen Freiheiten in den USA und Bemühen um Beschwichtigung des iranischen Monarchen blieb letztlich ungelöst. ${ }^{199}$ Trotz des spürbaren Drucks auf die oppositionellen Studenten durch US-Behörden waren Demonstrationen und diverse Aktionen an der Tagesordnung, wann immer ein Mitglied der kaiserlichen Familie in die USA reiste. Angesichts der öffentlichen Desavouierung waren die Zwillingsschwester des Schahs, aber auch andere Verwandte, wiederholt gezwungen, auf USA-Reisen zu verzichten. ${ }^{200}$

Angesichts der neuen „unabhängigen Politik“ Irans und der persönlichen Einstellung des Schahs gegenüber der bisherigen Schutzmacht kann es nicht überraschen, dass sich der Wandel in den beiderseitigen Beziehungen in allen Politikfeldern bemerkbar machte, wobei der generelle Trend hin zu einer weitgehenden Beseitigung auch der verbliebenen amerikanischen Einflussmöglichkeiten ging. Abseits der ab 1964 die Gesamtbeziehungen beherrschenden Verhandlungen über die Militärkredite und das Aufrüstungsprogramm reduzierten sich die verbliebenen amerikanischen Aktivitäten rapide - das bilaterale Verhältnis glich sich mehr und mehr dem zwischen gleichrangigen Staaten an. Bereits vor dem Amtsantritt Johnsons hatte die vormals herausragend wichtige Wirtschaftshilfe der USA an Iran weitgehend an Bedeutung verloren. Das im Anschluss an die Debatten des Clay-Committee stark reduzierte Auslandshilfe-Budget machte Einsparungen unausweichlich - Iran war eines der Länder, in denen die ökonomischen Hilfsprogramme so rasch als möglich eingestellt werden sollten. ${ }^{201}$

11; die Vorwürfe des Schahs über eine ägyptische Involvierung sind nicht völlig von der Hand zu weisen. Heikal zufolge wurden u.a. Ġotbzādeh wie auch dem späteren Außenminister der Bāzargān-Regierung 1979, Ebrāhim Jazdi, gemeinsam mit Palästinensern, Eritreern und anderen Afrikanern eine Kampfausbildung in Ägypten zuteil. Radiopropaganda gegen den Schah verweigerten sie allerdings und reisten anschließend in die USA oder in den Libanon weiter. Vgl. HEIKAL, Return of the Ayatollah, S. 71.

${ }_{198}$ Zentrale Organisation der oppositionellen iranischen Studenten in den USA war die 1952 gegründete Iranian Students Association, die sich an der Politik der Nationalen Front orientierte und stark regimekritisch eingestellt war. Vgl. Note, 3.6.65, ,POL 13-2-b [...]65', Lot 69 D 489, NEA/IRN: 1964-66, Box 11; vgl. SHANNON, Losing Hearts and Minds, S. 46-60, 78-89.

${ }^{199}$ Zur kommunistischen Finanzierung vgl. CIA Memo, OCI No. 1582/64, 30.5.64, FRUS 64-68, XXII, S. 61ff.; angeblich war zumindest Ġotbzādeh zwischenzeitlich vom KGB rekrutiert worden, allerdings ohne je wirklich für die UdSSR operativ tätig geworden zu sein. Vgl. ANDREW/MITROKHIN, Mitrokhin Archive II, S. 535 n78; KUZICHKIN, Inside the $K G B$, S. 302; GTI unterbreitete diverse Vorschläge, wie man eine Versöhnung zwischen dem Regime und den Auslandsstudenten herbeiführen könne, vorwiegend auf die Zusage entsprechender zukünftiger Karrieren im nun wirtschaftlich erfolgreichen Iran abzielend. Vgl. McLaughlin Memo, 19.11.65, ,EXD 12 [...]65", Lot 69 D 426, NEA/IRN: 1964-66, Box 8; DCI Helms versicherte dem Schah persönlich, dass die CIA die iranischen Studenten nicht finanziere. Vgl. Helms Memo, 23.8.67, FRUS 64-68, XXII, S. 419f.

200 Ašraf war wegen der Studentenproteste mitunter gezwungen, inkognito zu reisen. Vgl. Tehran \#626/\#1151, 21.10.65/19.2.66, POL 7 IRAN, CF 64-66; für einen Überblick über die Studentenproblematik vgl. SHANNON, Contacts With the Opposition; der SAWAK versuchte, durch Druck auf die in Iran verbliebenen Nationalisten eine Einstellung der politischen Aktivitäten der Studenten im Ausland zu erreichen. Vgl. CAS $\{C I A\}$ Memo, 1.8.64, Asnād 22, 3, S. 17 f.

${ }^{201}$ Vgl. Komer Memo, 17.1.64; Bell Memo, 21.1.64, Rostow Memo, 14.10.66; Hamilton Memo, 1.12.66, FRUS 64-68, IX, S. 5, 6f., 161-4, 167-74; AID Administrator Bell hatte Johnson Anfang 1964 die umgehende Einstellung der neun erfolgreichsten Hilfsprogramme empfohlen, da diese Staaten inzwischen weitgehend auf eigenen Füßen stehen könnten. Unter den genannten Staaten waren 5 nahöstliche, zu diesem Zeitpunkt aber nicht Iran, dessen Programm aber als ,auslaufend“ definiert wurde. Der letzte Direktor der Mission in Teheran war Edward F. 
NEA hatte kurzzeitig energischen Widerstand geleistet und die Fortsetzung des iranischen Programms gefordert, da das Land weiterhin ,tragically weak" sei und jederzeit eine neue Krise drohen könne - angesichts der steigenden Öleinnahmen war diese Position aber kaum aufrechtzuerhalten. ${ }^{202}$ In NEA erwartete man angesichts der Einstellung der direkten Wirtschaftshilfe und der Entwicklungsdarlehen eine grundsätzliche Transformation des bilateralen Verhältnisses: „[...] we shall be drawn less closely into the government's future decision making process and shall probably adopt more nearly the role of trusted ally rather than that of responsible senior partner." ${ }^{203}$ Man müsse daher versuchen, auch für die Zeit nach Ende der Wirtschaftshilfe amerikanischen Einfluss aufrechtzuerhalten, vorwiegend durch starke Involvierung der amerikanischen Industrie im aufstrebenden iranischen Markt während der „Übergangsphase“.

Bis zur vollständigen Einstellung jeder Hilfe im Rahmen des United States Foreign Assistance Act dauerte es letztlich bis zum 30. November 1967, dem Tag, an dem die US AID Mission in Teheran geschlossen wurde. ${ }^{204}$ Damit war eine historisch signifikante Methode der USA zur Einflussnahme in Iran aufgegeben ${ }^{205}$ - seit 1952 hatten die USA insgesamt \$605 Millionen an Wirtschaftshilfe zur Verfügung gestellt. Zusätzlich waren Nahrungsmittelüberschüsse im Rahmen des PL-480-Programms im Wert von \$122 Millionen geliefert worden. Die ExportImport-Bank hatte zudem zwischen 1955 und 1968 Darlehen in Höhe von ca. \$240 Millionen gewährt. Nicht in Zahlen messbar war der durch die umfassende Wirtschaftshilfe und die damit einhergehende Involvierung in die Planungs- und Entscheidungsprozesse des Empfängerlandes erzeugte strategische Einfluss der USA und der Aufbau pro-amerikanischer Netzwerke in Iran. So waren allein 1968 die iranischen Außen-, Finanz- und Innenminister allesamt ehemalige lokale Beschäftigte von US AID. ${ }^{206}$ Secretary of State Dean Rusk kommentierte die Einstellung der Hilfe öffentlich mit der lapidaren Feststellung: ,[...] the story of modern Iran is one of the great success stories of our time. “207

\footnotetext{
Tennant. Vgl. Saunders Memo, 23.1.64, FRUS 64-68, XXII, S. 9; Vgl. Macy Memo, 13.4.66, ,April, 1966 [1 of 2]', Special Files, Handwriting File, Box 14, LBJL.

202 Jernegan Memo, 19.3.64, ,AID 1 [...]64 64, Lot 69 D 30, NEA/IRN: 1964-66, Box 2; vgl. Jernegan Memo, 20.3.64, FRUS 64-68, XXII, S. $21 \mathrm{f}$.

${ }_{203}$ Howison Memo, 18.1.65, ebd., S. 125; Howison warnte, dass man die bis zum Auslaufen verbliebenen Programme nutzen sollte, um den Eindruck einer allein auf das Militärische begrenzten Unterstützung der USA zu vermeiden.

${ }_{204}$ Wenig überraschend war man seitens der Botschaft über die Einstellung des Hilfsprogramms und den damit verbundenen Einflussverlust entrüstet. Vgl. Tehran \#2118, 11.11.66, AID (US) 8 IRAN, CF 64-66; AID kam den Wünschen der Botschaft nach einem ,decent burial of the AID program" entgegen und verhinderte eine überhastete Schließung Ende Juni 1967 und ermöglichte das Auslaufen einiger Programme innerhalb des Finanzjahres 1968. Den von der Botschaft vorgeschlagenen ,national interest waiver“ durch den Präsidenten verweigerte man allerdings. Vgl. Meyer Letter, 15.9.66; Macomber Letter, 18.10.66, ,AID 1 Gen. Policy 1966“, Lot 69 D 513, NEA/IRN: 1964-66, Box 14.

${ }^{205}$ Meyer bedauerte den einhergehenden Verlust infolge des Abzugs der durch die AID-Leistungen finanzierten Berater, z.B. der US-Berater in der Zentralbank: "These, it seems to me, are the sort of advisors who can be invaluable in their direct and significant impact on present and future Iranian practices and policies."; ebd.

${ }^{206}$ Insgesamt \$225 Millionen der Unterstützung erfolgte in Form zurückzahlbarer Darlehen. Die ExIm-Bank hatte zudem etwa $\$ 50$ Millionen an Exportbürgschaften geleistet. Vgl. Administrative History: Iran, S. 12f., 14; hinzu kam die ausgeweitete Tätigkeit des Peace Corps mit 260 Freiwilligen in Iran im Jahr 1968.

${ }^{207}$ State Press Release, 29.11.67, ,Near and Middle East 1965-66', Lot 72 D 139, S/P Records, Subject and Country: 1965-69, Box 315 .
} 
So sehr man sich in der Retrospektive bemühte, das iranische „Wirtschaftswunder“ der 1960er Jahre im Nachhinein als eigenen Erfolg darzustellen, so war doch offenkundig, dass der weitgehende Ausschluss amerikanischen Einflusses und die rasche wirtschaftliche Erholung fast zeitgleich vonstatten gingen. Der 3. Entwicklungsplan, dieses Mal auf fünfeinhalb Jahre angelegt, verfolgte die Industrialisierung Irans im Zuge einer Importsubstitutionspolitik, im Grunde ein Ansatz, der seitens der amerikanischen Berater in den Vorjahren immer abgelehnt worden war. Statt der Planorganisation mit ihren vielen in den USA ausgebildeten Mitarbeitern und Mitgliedern des ehemaligen pro-amerikanischen Netzwerkes in Iran war nun das im Februar 1963 durch den Zusammenschluss anderer Ministerien geschaffene und weitgehend autonome Wirtschaftsministerium unter dem in Frankreich ausgebildeten Minister 'Alinagi 'Ālixāni für die eigentliche Wirtschaftsplanung zuständig. Insbesondere die enge Zusammenarbeit mit dem Ostblock beim Aufbau iranischer Schwerindustrien - herausragend war dabei die Vereinbarung über den Stahlhüttenbau - durch 'Ālixāni war dabei kaum im breiteren amerikanischen Interesse. ${ }^{208}$ Wirtschaftsplanung - vormals zentraler amerikanischer Einflussort im Klientelstaat - erfolgte Mitte der 1960er in vielerlei Hinsicht ohne den zuvor dominierenden ideellen Input aus den USA. Bill Miller beschwerte sich frustriert über die nun untergeordnete Rolle der Planorganisation, obwohl aufgrund der Öleinnahmen erstmals ausreichend Investitionskapital zur Verfügung stünde. ${ }^{209}$ Dennoch feierte man die rasante Wirtschaftsentwicklung. Tatsächlich stieg die Wirtschaftsleistung Irans zwischen 1963 und 1968 um etwa 7\% pro Jahr - in der zeitgenössischen Literatur war damals sogar die Rede von (nominalen) Wachstumsraten in Höhe von durchschnittlich 9,8\% im selben Zeitraum. ${ }^{210}$ Obgleich überzogen, so waren die von der iranischen Regierung verbreiteten Zahlen doch Ausdruck der vom Schah verkündeten Zielsetzung, Iran in einen ,,show-case of modernization in this part of the world" $\mathrm{zu}$ verwandeln. ${ }^{211}$

\footnotetext{
${ }^{208}$ Die Planorganisation wurde mit Verabschiedung des 3. Entwicklungsplans im September 1962 ,,reorganized into a planning and supervisory rather than an executing agency."; Tehran \#A-493, 24.3.65, FN 15 IRAN, CF 6466; für eine etwas überzogene Interpretation des Niedergangs des autonomen Wirtschaftsministeriums bis Ende der 1960er Jahre infolge des US-Widerstands vgl. NASR, Late-Pahlavi State, 1 (2000), S. 106ff.; tatsächlich hatten 36\% der Minister in den Kabinetten von 1963 bis 1979 ihre Ausbildung in Frankreich genossen, 26\% in den USA. Dies ist allerdings eher Ausdruck des tradierten starken französischen Einflusses auf das Bildungswesen in der Vergangenheit als Ausdruck des politischen Einfluss Frankreichs. Tatsächlich hatte selbst die Bundesrepublik Deutschland einen größeren ökonomischen Stellenwert für Iran als die V. Republik. Vgl. AzIMI, Quest for Democracy, S. 227; 'Ālixāni bemühte sich andererseits energisch um westdeutsche Investitionen. Vgl. EinzelInformation Nr. 417/69, 2.5.69, BStU, MfS, HV A, Nr. 145; der Bundesverband der Deutschen Industrie berichtete im Juli 1968 über die Gelegenheit, die Stellung Westdeutschlands als wichtigster Handelspartner Irans sogar noch auszubauen. Vgl. Einzel-Information Nr. 862/68, 21.8.68, BStU, MfS, HV A, Nr. 133; für den breiteren Kontext der Umorientierung in der wirtschaftspolitischen Strategie Irans vgl. NASSEHI, Iranian Developmentalism, S. 5060.

${ }^{209}$,With considerably more oil revenues to spend than in Ebtehaj's time, far less is being done by the Plan Organization“; Miller drängte (vergeblich) auf neue Initiativen nach dem Muster der Task Force-Politik Anfang der Dekade: „Men of ability abound in Iran, but almost none are allowed to the circles of power.“; Miller Letter, 11.2.64, POL 7 IRAN, CF 64-66.

${ }^{210}$ Z.B. in RAMAZANI, Changing Foreign Policy, S. 426 n6; durchschnittlich 7\% Wirtschaftswachstum wurden in der abschließenden Administrative History erwähnt. Vgl. Administrative History: Iran, S. 1; AID schätzte z.B. das Wachstum von 1964 bis 1966 auf 5-6\% und hielt die Angaben der Iraner für das Jahr 1966 in Höhe von 10,7\% für überzogen. Vgl. Wriggins Memo, 24.6.66, ,Iran, Memos \& Misc, Vol. 2 [2 of 2]', NSF, Country File, Box 136, LBJL; vgl. auch MCLACHLAN, Iranian Economy, S. 141.

211 Tehran \#1572, 23.5.66, angehängt an Rostows Memo, FRUS 64-68, XXII, S. 255; weder Botschaft noch internationale Organisationen schenkten den vorwiegend politisch motivierten Zahlenangaben der Iraner Glauben.
} 
Offenbar gab es auch innerhalb der politischen Elite Irans dennoch verbreitete Spekulationen über verdeckte Aktivitäten der USA. Prägend war hier letzten Endes die Beteiligung am Staatsstreich von 1953, der in der iranischen politischen Mythologie die Idee amerikanischer Omnipotenz im Arkanbereich installierte. So sahen iranische Offizielle, unter anderem der SAWAK-Direktor, den Finanzminister und späteren Ministerpräsidenten (1977-78) Ğamšid Āmuzegār offenbar als Erfüllungsgehilfen amerikanischer Politik. Ein Offizieller nannte ihn unverhohlen einen „Agenten“. ${ }^{212}$ Dies war wohl eher eine Übertreibung, doch war nicht von der Hand zu weisen, dass auch nach der Suspendierung des reformistischen Interventionismus Bemühungen unternommen wurden, die nun Jahrzehnte alte amerikanische Position in Iran auch zum eigenen Vorteil zu nutzen. Tatsächlich hatte Āmuzegār wenigstens in einem Fall zugunsten übergeordneter amerikanischer Interessen interveniert. ${ }^{213}$ Mit der weitgehenden Ausschaltung der Planorganisation als Steuerzentrale und dem Ausschluss amerikanischen Einflusses aus dem temporär zentralen Wirtschaftsministerium konzentrierten sich die Überreste des pro-amerikanischen Netzwerks auf andere Behörden und Ministerien, insbesondere auf die Zentralbank unter Mohammad Mehdi Sami i i und dem stellvertretenden Gouverneur Xodādād Farmānfarmāeijān. ${ }^{214}$ Ähnlich wie früher die Planorganisation engagierte sich auch die Zentralbank weit jenseits des ihr zugeschriebenen Aufgabenbereichs und war mit ihrer Schwerpunktsetzung auf ökonomische Stabilität und Effizienz im Grundsatz den alten reformistischen Zielsetzungen der USA verschrieben. Ein Botschaftsbericht pries die Zentralbank als ,most enlightened and energetic of any economic agency of the government of Iran“, verwies aber zugleich auf die wachsenden Widerstände gegen deren Politik, gespeist nicht zuletzt von starkem Misstrauen anderer Ministerien gegenüber der pro-amerikanischen Ausrichtung und der Tatsache, dass sowohl Sami`i wie auch Farmānfarmāeijān ehemalige Protegés Ebtehāğs waren. ${ }^{215}$

\footnotetext{
Vgl. MemCon, 30.6.65, FN 12 IRAN, CF 64-66; zu den Manipulationen der öffentlichen Statistiken vgl. AMINI, Single Party State, S. 133-6.

${ }^{212}$ So Mehdi Pirāsteh, der Botschafter Irans in Bagdad, der als möglicher Howejdā-Nachfolger gehandelt wurde, gegenüber dem Embassy Counselor Martin Herz. Der anwesende Pākrawān habe zustimmend genickt, so Herz später. Vgl. MemCon, 11.3.65, ,Political Affairs \& Rel. IRAN/IRAQ IRAN 1965‘, Lot 69 D 482, Box 9; MemCon, 24.4.65, ,INF 1 General Policy. Iran 1965', Lot 69 D 426, NEA/IRN: 1964-66, Box 8; offenbar betrachteten die Sowjets den späteren, als pro-amerikanisch geltenden iranischen Botschafter Hušang Ansāri ebenfalls als „CIA agent“. Vgl. NASR, Late-Pahlavi State, S. 108; Bill Miller kommentierte Jahre später: ,the interests of Iran, from a purely patriotic Iranian point of view, were sufficient to....in most cases, to have a close relationship with the United States.“; William G. Miller OHI, 25.3.85, IOHP, Transcript 4, S. 25.

213 Auf amerikanisches Bitten hin hatte er die Umsetzung einer Instruktion des Schahs über die Militärkreditverhandlungen verzögert. Vgl. Handley Memo, 8.11.65, ,AID 15-11 [...]65', Lot 69 D 426 , NEA/IRN: 1964-66, Box 8.

${ }^{214}$ Ein weiteres Mitglied des verbliebenen pro-amerikanischen Netzwerks, 'Abdo r-Rezā Ansāri, schilderte z.B. im April 1965 gegenüber Martin Herz Pläne für ein neues Kabinett unter Leitung von Āmuzegār und ihm selbst als stellvertretendem Ministerpräsidenten sowie pro-amerikanischen Parteigängern wie Sami 'i, Farmānfarmā ijān oder auch Habib Nafisi als Ministern. Offenkundig hoffte Ansāri auf Washingtons Unterstützung für eine Kabinettsumbildung - erst in der Krise von 1977 wurde Āmuzegār mit der Kabinettsbildung beauftragt, dann auch offenkundig von Mohammad Rezā als Geste gegenüber den USA gedacht. Vgl. MemCon, 17.4.65, ,POL 1 Gen. Policy. Background. 1965', Lot 69 D 490, NEA/IRN: 1964-66, Box 12, RG 59, NACP; Lilienthal nannte die Gruppe ,that group of younger men who are now running the country - or trying to.“; Eintrag vom 18.4.64, in: LILIENTHAL, Journals: VI, S. $29 \mathrm{f}$.

${ }^{215} \mathrm{Vgl}$. Tehran \#A-38, 22.7.64, FN 6 IRAN, CF 64-66.
} 
Mit dem Auslaufen der Wirtschaftshilfe im engeren Sinne nahm amerikanischer Einfluss zwangsläufig ab. Bemühungen, den früheren beherrschenden Einfluss durch Aktivitäten in anderen Feldern auszugleichen, stellten sich rasch als Wunschdenken heraus. ${ }^{216} \mathrm{Zu}$ erwartende Produktionsausfälle im iranischen Agrarsektor infolge der Landreform und steigende Nachfrage auf dem Land wurden als vielversprechende Gelegenheiten zugunsten der Stärkung des US-Einflusses in der Zukunft ausgemacht, eine ältere Idee des amerikanischen Strategiepapiers vom Mai 1963 wieder aufgreifend: „These opportunities not only offer potential commercial advantages, they also provide what may turn out to be one of our chief means of maintaining U.S. influence in this strategically located country. “217 Angesichts der neuen iranischen Finanzkraft wandelte sich aber die Nahrungsmittelhilfe an Iran im Rahmen des PL-480-Programms bald in eine Maßnahme zur Verbesserung der amerikanischen Handelsbilanz. Die vertraglichen Verpflichtungen zum Einkauf von Produktionsgütern in den USA wurden ausgedehnt und bereits im September 1964 wurde auch hier von Zuschüssen auf Darlehensgewährung umgestellt. ${ }^{218}$ Zudem hatte man den Iranern im Kontext der Verlegung der Abhörstationen Entgegenkommen bezüglich eines mehrjährigen PL-480-Abkommens zugesagt. Allerdings überwogen in der Umsetzung bürokratische Trägheit und kommerzielle Interessen auf amerikanischer Seite. Statt einer Basis für neuerlichen Einflussgewinn der USA in Iran tendierte das PL-480-Programm eher zu einem Nebenschauplatz, wenn nicht sogar teilweise zu einer Belastung der bilateralen Beziehungen. ${ }^{219}$

Auf der zwischengesellschaftlichen Ebene machte sich die Tätigkeit des von John F. Kennedy ins Leben gerufenen Peace Corps bemerkbar. Ab 1962 begab sich eine wachsende Zahl von jungen idealistischen Amerikanern auf den Weg nach Iran und leistete in verschiedensten Lebensbereichen freiwilligen Hilfsdienst ab, gerade in ländlichen Gebieten. 1968 hatte die Zahl der Freiwilligen mit 260 einen vorläufigen Höchststand erreicht, 1976 wurde das Programm

216 Auf großes kommerzielles US-Interesse stieß ein iranischer Vorschlag zur Beteiligung am Elektrifizierungsprogramm mit Kosten von \$200 Millionen. Reste des pro-amerikanischen Netzwerks wurden zur Förderung dieser Interessen eingestezt, insbesondere die Schlüsselstellung des Zentralbankchefs Mehdi Sami `i im Entscheidungsprozess: ,extensive delays could endanger Samii's control of this program and our chances of obtaining this rich plum."; Walsh Memo, 17.6.64, ,AID 9-b Master Electrification IRAN 1964“, Lot 69 D 30 , NEA/IRN: 1964-66, Box 2; bezeichnend ist, dass Sami' $\mathrm{i}$ rasch aus dem Entscheidungsprozess auf iranischer Seite gedrängt wurde. Vgl. Swihart Letter to Walsh, 20.7.64, ebd.; David Lilienthals Development and Resources Corporation in Xuzestān hatte zwar 1962 einen dritten Vertrag mit der Regierung abgeschlossen und im Rahmen des Projekts wurde noch 1963 der Dez-Damm vom Schah selbst eingeweiht. Das US-Unternehmen hatte aber nur begrenzten Einfluss auf die Planungen, trug aber dennoch Verantwortung für die gravierenden ökologischen Folgen des Dammbaus. 1971 wurde die Firma von einem Rockefeller-Unternehmen aufgekauft, 1979 ging sie infolge der Revolution in Iran bankrott. Vgl. die Einträge vom 12.7.64, 29.6.65, in: LILIENTHAL, Journals: VI, S. 52f., 144f.; und den Eintrag vom 7.1.79, in: LILIENTHAL, Journals: VII, S. 741f.; vgl. FISHER, Lilienthal, S. 445ff.; EkBladi, Profits of Development, S. 503ff.; BILL, Eagle and Lion, S. $358 \mathrm{f}$.

${ }^{217}$ Bracken Memo, 22.10.64, FRUS 64-68, XXII, S. 106.

${ }^{218}$ Vgl. State \#186, 7.8.64, AID (US) 15-8 IRAN, CF 64-66.

${ }^{219}$ Aufgrund der vielen Verzögerungen der Getreidelieferungen warnte Meyer, dass die Schwierigkeiten des PL480-Programms sich zunehmend negativ auf die bilateralen Beziehungen auswirkten. Siehe Meyer Letter, 22.9.65, Handley Memo, 8.11.65, ,AID 15-11 [...]65', Lot 69 D 426, NEA/IRN: 1964-66, Box 8; tatsächlich war Iran das erste Land überhaupt, das mit den USA ein PL-480-Abkommen auf Title IV-Basis, also auf Basis von Verkäufen auf Kreditbasis für US-Dollar, abschloss. Diese dienten in erster Linie dem (subventionierten) Verkauf von amerikanischen Überschüssen, also kommerziellen Interessen. Vgl. Tiger Letter, 8.2.65, , AGR 7 Visits Iran 1965', ebd.; vgl. Tehran \#1733/\#108, 25.6./9.7.66, ,Wriggins Memos, 1966“, NSF, Name File, Box 8, LBJL; positiver gestaltete sich die Zusammenarbeit auf dem Gebiet der Wasserversorgung. Im März 1968 wurde ein entsprechendes Kooperationsabkommen unterzeichnet. Vgl. Administrative History: Iran, S. 25f. 
dann eingestellt. ${ }^{220}$ Auch die Zusammenarbeit auf dem Gebiet der technologischen Kooperation und im Bereich der Wissenschaft wurde in der Folgezeit intensiviert. Ein Notenaustausch im Mai 1968 formalisierte die Kooperation. Von großer Bedeutung für die fernere Zukunft wurden zudem die Vereinbarungen im Bereich der zivilen Nutzung der Atomenergie. Das erste Abkommen aus dem Jahr 1957 wurde 1964 und 1967 erweitert und legte die Grundlagen für die bald ambitionierten Pläne Mohammad Rezās für die Nutzung der Atomkraft. $^{221}$

Vielversprechender erschien, die Einstellung der eigenen Hilfen durch Ausweitung des wirtschaftlichen Austausches auf dem Privatsektor auszugleichen, ,[...] to insert means of continuing a high degree of United States involvement in Iran's growth process. “222 Unterstützt von der Export-Import-Bank - sie vergab allein zwischen 1963 und 1968 \$173 Millionen an Darlehen für industrielle Projekte mit Beteiligung amerikanischer Unternehmen und $\$ 50$ Millionen an Bürgschaften für amerikanische Exporte - versuchte die US-Administration, Investoren nach Iran zu locken. Kommerzielle Investitionen amerikanischer Unternehmen beliefen sich bis Mitte 1967 auf eine Größenordnung von etwa \$300 Millionen. ${ }^{223}$ Schwierigkeiten für amerikanische Investoren ergaben sich allerdings durch die weitverbreitete Korruption innerhalb Irans, insbesondere die Aktivitäten von Mitgliedern der kaiserlichen Familie, ,[...] some of whom have had their fingers in quite a number of different commercial ventures in Iran“. ${ }^{224}$ So hätten Geschäftsbeziehungen zwischen amerikanischen Unternehmen und Mitgliedern der kaiserlichen Familie wiederholt zu Belastungen der diplomatischen Beziehungen geführt. Ein seitens der Iraner unilateral storniertes forstwirtschaftliches Projekt führte zu einer Klage des betroffenen Unternehmens in den USA und zu unwillkommener Aufmerksamkeit seitens des US-Kongresses. Zwischenzeitlich drohte sogar die Suspendierung der gesamten US-Auslandshilfe an Iran infolge des Rechtsstreits. ${ }^{225}$ Mit dem rasanten Anstieg

\footnotetext{
${ }^{220}$ Vgl. Administrative History: Iran, S. 14; vgl. EhSANI-NiA, Public Diplomacy; Rostam-KolaYi, Peace Corps in Iran.

${ }_{221}$ Vgl. Administrative History: Iran, S. 25ff.; besonderes Interesse zeigte der Schah für den Aufbau einer modernen Universität in Schiras mit Unterstützung von AID - als Zeichen für seine Ziele ernannte er den früheren Ministerpräsidenten 'Alam zum Rektor. Vgl. Briefing Paper, ,Pahlavi University“, 1.6.64, ,POL 15-1A', Lot 69 D 178, NEA/IRN: 1964-66, Box 4; zur Kooperation im Bildungssektor vgl. LESLIE/KARGON, Exporting MIT; zu den nuklearen Ambitionen vgl. PATRIKARAKOS, Nuclear Iran, S. 14-28; KiBAROGLU, Iran's Quest; ders., Iran's Nuclear Ambitions.

${ }^{222}$ Administrative History: Iran, S. 16.

${ }^{223}$ Ebd., S. 22; allerdings waren etwa 90\% der US-Investitionen auf den Ölsektor konzentriert. Vgl. Tehran \#A$414,8.2 .68$, FN 9 IRAN-US, CF 67-69.

${ }^{224}$ Stave Letter to Lill, 28.9.65, , INCO Chem[...]65`; Memo for the Record, 20.7.65, ,INT 2 [...]65', Lot 69 D 482, NEA/IRAN: 1964-66, Box 9; der Halbbruder des Schahs, Prinz 'Abdorrezā, bestätigte in einem vertraulichen Gespräch die Korruption innerhalb der Familie. Siehe Memo for the Record 11.6.64, ohne Folder, Lot 69 D 225 , NEA/IRN: 1964-66, Box 6; die von Außenstehenden häufig als zentraler Austragungsort der Korruption innerhalb der kaiserlichen Familie verdächtigte, 1961 vom Schah gegründete Pahlavi Foundation zeichnete sich eher durch fehlgeleitete Investitionen aus. Die Botschaft befand die Stiftung ,not particularly impressive [...] and certainly not at all sinister."; ein Dokument von 1978 bemerkt allerdings, dass zwischen 20 und $40 \%$ der Einnahmen der Stiftung an diverse kaiserliche Familienangehörige gehen würden. Vgl. Tehran \#A-19, 12.7.66, INCO 6 IRAN, CF 64-66; Annex C zu Tehran \#A-105, 20.6.78, Asnād 7, S. 226; zu Ašraf vgl. auch AzIMI, Quest for Democracy, S. 237f., 244ff., zur allgemein weit verbreiteten Korruption, die US-Investoren zuweilen abschreckte. Vgl. auch SHAwCross, Shah's Last Ride, S. 192-5; PRECHT, A Diplomat's Progress, S. 108-14.

${ }^{225}$ Das Fairhurst-Unternehmen hatte mit einem öffentlichen Unternehmen in der Provinz Gilān im Mai 1964 eine Vereinbarung zur forstwirtschaftlichen Nutzung von Waldgebieten entlang des Kaspischen Meeres abgeschlossen.
} 
der Öleinnahmen stieg wenig überraschend auch das Interesse amerikanischer Investoren. Eine frühe Version des unten analysierten National Policy Paper für Iran hatte propagiert, man ,[...] would foolishly sacrifice our own national interest if we did not preserve for United States industry an appropriate share of the growing commercial market for capital goods which we helped build up through soft loans in the past.“226 Tatsächlich wurde privatwirtschaftliches Engagement im großen Stil als zentraler Ersatz für amerikanischen Einfluss in ,post-AID countries“ angesehen. $^{227}$ Die Johnson-Administration unterstützte den Privatsektor und einflussreiche Offizielle mit gleichermaßen guten Beziehungen zur amerikanischen Industrie wie zum kaiserlichen Hof wie Averell Harriman oder David Rockefeller übernahmen die Aufgabe, die entsprechenden Kontakte herzustellen. ${ }^{228}$

Bis weit in die 1970er Jahre war das Gros privatwirtschaftlichen Engagements in Iran allerdings den Aktivitäten der Ölindustrie geschuldet. Die Beziehungen des Konsortiums zur iranischen Führung waren zwar nicht spannungsfrei, im Vergleich zu denjenigen zwischen Produzenten und Konzernen in anderen Staaten jedoch erstaunlich ausgeglichen. Mit der Gründung der OPEC und dem Erscheinen von Independents verschärften sich die Spannungen innerhalb der nahöstlichen Erdölordnung, was auch nicht ohne Auswirkungen auf das Verhältnis zu Iran blieb. Die Aussicht auf Konflikte rief alsbald die Politik auf den Plan. Die KennedyAdministration hatte sich anfangs britischen Forderungen entzogen, stärker auf die Politik der großen Ölkonzerne im Sinne der eigenen nationalen Sicherheit einzuwirken. ${ }^{229}$ Gleichzeitig war man sich des auch langfristigen Problems bewusst, das sich mit der einseitigen Abhängigkeit der westlichen Staaten vom nahöstlichen Erdöl ergab. ${ }^{230}$ Nach dem OPEC-

Der Kontrakt wurde ohne Angabe von Gründen im Januar 1965 annulliert. Vgl. Talbot Memo, 13.7.65; Saunders Memo, 12.7.65, ,Iran, Memos [...]12/65', NSF, Country File, Box 136, LBJL.

${ }^{226}$ First ,Regular' Draft National Policy Paper on Iran, 26.11.65, ,POL 1-2 [...]65', Lot 69 D 490, NEA/IRN: 1964-66, Box 12.

227 „One of the important questions of the 1970's will be: Do we have the imagination to put together the next generation of economic relationships - the relationships that naturally grow out of all our work in economic aid since the Marshall Plan? Or having done our aid job well, are we going to lose out politically because we couldn't follow up?"; Rostow Memo, 23.7.68, ,Rostow Memos [1 of 2]", NSF, Name File, Box 7, LBJL; eine besondere Befürchtung für die Nahostregion war die Aussicht auf Einflussabnahme der USA aufgrund der Tatsache, dass diese die ,first major region of the developing world which nears meeting its capital needs from its own resources“ sei; Memo ,The U.S. in the Broader Middle East", 9.3.70, DDRS: CK3100707364; laut BASHIRIYEH, State and Revolution, S. $28 n 48$, waren die USA für 54\% der Auslandsinvestitionen in Iran zwischen 1962 und 1967 verantwortlich.

${ }^{228} \mathrm{Zu}$ Harrimans Vorgehen während dem Staatsbesuch des Schahs im August 1967 vgl. Administrative History: Iran, S. 19ff.; zum Tehran Investment Seminar und Rockefellers Rolle vgl. BILL, Eagle and Lion, S. 180f.; auf Rockefeller folgte ein weiterer Besuch der Establishment-Ikone John McCloy. Vgl. Harriman Letter, 2.11.67, ,Ansary, Hushang, 1967-68‘, Box 432, Harriman Papers, LOC; über die Rolle ausländischer Direktinvestitionen siehe RAHNEMA, Multinationals; ein ausführliches Beispiel für die vielfältigen Schwierigkeiten für amerikanische Investoren in Iran bei BLASZCZYK, Challenges to Multinationals; die Botschaft erstellte 1972 eine sehr eingehende und einfühlsame Analyse der Korruptionsproblematik in Iran. Vgl. Tehran \#A-105, 20.6.72, Asnäd 17, S. 74-89. ${ }_{229}$ So in anglo-amerikanischen Konsultationen vom Februar 1961. Vgl. MemCon, 13.2.61, FRUS 61-63, IX, S. 752-4; interessant in diesem Gespräch ist die Erwähnung der eigenständigen italienischen Ölpolitik. Ein USOffizieller fragte, , , [...] if the UK had had any thoughts on how to bring the Italian concern Mattei back under control. This firm was very useful to the Soviets as a means of breaking into Western oil markets. Lord Hood replied in the negative."; ebd., S. 754.

${ }^{230}$ Das Interdepartmental Committee of Under Secretaries on Foreign Economic Policy hatte Ende 1961 die Weltölsituation erörtert: , [...] not only does the Free World get two-thirds of its oil from the Middle East now but that 50 years from now it will probably be even more dependent on Middle East supplies. From the security standpoint of our longer-term interest in the broadest sense, we must consider what kind of arrangements we can work out to insure Free World access to Middle East oil."; Summary Minutes of Meeting, 13.12.61, ebd., S. 773; 
Treffen vom Juni 1962 hatte sich der Ton zwischen Förderländern und Ölkonzernen verschärft - die Produzenten einigten sich erstmals auf eine gemeinsame Position in der Frage der steuerlichen Behandlung der Abgaben. ${ }^{231}$ Iran blieb anfangs seiner tradierten „pro-westlichen“ Ölpolitik treu und verweigerte sich der von anderen OPEC-Mitgliedsstaaten gewünschten Einführung von Produktionsquoten. ${ }^{232}$ Nachdem sich aber zunehmend abzeichnete, dass die Ölkonzerne die Forderungen der OPEC zu ignorieren beabsichtigten und auf eine bilaterale Verhandlungsstrategie gegenüber den einzelnen Produzenten setzten, schien sich die Haltung Teherans zu ändern. Der erste Generalsekretär der OPEC, gleichzeitig stellvertretender Direktor der NIOC, Fu'ād Ruhāni, erzwang als offizieller Verhandlungsführer die symbolische Anerkennung der OPEC durch die Konzerne. Die Krise zwischen OPEC und den Konzernen spitzte sich Ende $1963 \mathrm{zu}$ - der seitens der US-Ölmagnaten befürchtete „real warfare“ schien unmittelbar bevorzustehen. ${ }^{233}$

Angesichts der zunehmend prekären Situation in den Verhandlungen zwischen Industrie und nahöstlichen Regierungen und möglichen strategischen Konsequenzen wurde auch die seit Entstehung des iranischen Ölkonsortiums konsequent durchgehaltene Nichtintervention der US-Administration aufgeweicht. In den anglo-amerikanischen Ölgesprächen vom Juni 1963 kamen beide Regierungen überein, abgesehen von Ausnahmesituationen in die Verhandlungen zwischen Produzenten und Firmen nicht intervenieren $\mathrm{zu}$ wollen. ${ }^{234}$ Zudem versuchte man einer weiteren unerwünschten Aufwertung der OPEC entgegenzuwirken und lehnte direkte Gespräche mit der Organisation ab. Andererseits vereinbarte man, die eigenen Positionen, insbesondere diejenige gegenüber den eigenen nationalen Ölkonzernen, mit dem Verbündeten zu koordinieren, mit dem Ziel, die Verhandlungsposition der Majors zu konsolidieren. ${ }^{235}$ Nicht abschließend zu klären ist, inwieweit diese leicht modifizierte Haltung in der US-Ölpolitik zur

zudem ergäbe sich ein politisches und strategisches Problem aus der Dominanz der großen Ölkonzerne gegenüber den Förderländern. Die Konzerne seien ,,in a position to dominate completely the political life of the countries because the companies are the source of the bulk of the Governments' revenues."; ebd.; dem gegenüber stünde der zunehmende Wettbewerb durch neue Konkurrenten und der Druck der nahöstlichen Regierungen auf größere Profitbeteiligungen. Die Befürchtungen hinsichtlich OPEC nähmen ebenfalls zu. Im gleichen Meeting gab es auch erste Überlegungen, eine strategische Reserve einzurichten.

${ }^{231}$ Vgl. Tehran \#8, 2.7.62, ebd., S. 793f.; tatsächliche Preiserhöhungen waren Anfang der 1960er Jahre nicht wirklich realistisch, nicht zuletzt aufgrund der Effekte der sowjetischen Öloffensive auf den Ölpreis. Vgl. MemCon, 18.7.61, ebd., S. 762ff.; INR Research Memo Draft, Dezember 1963, ,[...]PET 3-b, OPEC‘, NEA/NE: Records of the Officer in Charge of Economic Affairs, 1947-1963, Box 10; McGhee forderte, die 50:50-Formel so lange wie möglich zu verteidigen, notfalls indem man auf die Spaltung der OPEC hinarbeite. Vgl. McGhee Memo, 20.7.62, ,6-A/1. O.P.E[...]', NEA, GTI: RIAD, 1958-63, Box 7; im Department plädierte man für Zurückhaltung. Vgl. Johnson Memo, o.D., ebd.

${ }^{232}$ Allerdings drängte man zugleich die US-Administration, die Ölgesellschaften zu Produktionssteigerungen in Iran zu bewegen. McGhee und andere Offizielle unternahmen in der Tat derartige Anstrengungen gegenüber den Majors. Vgl. Brubeck Memo, 18.8.62, ,Iran General 8/12/62-8/31/52‘, NSF, Countries, Box 116, JFKL.

${ }^{233}$ MemCon, 6.12.63, FRUS 61-63, XVIII, S. 829; Davies Memo, 16.9.63, FRUS 61-63, XVII-XVIII-XX-XXI Suppl., Dok. 13; noch im Mai 1962 hatte sich Ruhāni beim Konsortium für die Veröffentlichung einer Studie aus Petroleum Intelligence Weekly entschuldigt, welche die exorbitanten Profite ( 22 bis $64 \%$ des investierten Kapitals) der Konzerne offenbarte. Vgl. Tehran Joint Weekly \#WEEKA20, 21.5.62, 788.00(W)/5-2162, CF 60-63; Study ,The Arthur D. Little Report to OPEC', 4.5.62, ,Middle East - General, Jun to December 1962', Records of the Director (NE): 1958-1963, Box 7; zu Ruhāni vgl. MiLANI, Eminent Persians: I, S. 272-6.

${ }^{234}$ Vgl. Davies Memo, 5.7.63, FRUS 61-53, XVIII, S. 630-4.

${ }^{235}$ Vgl. ebd., S. 631; dies waren die ersten anglo-amerikanischen Ölgespräche seit 1960. Vgl. ed. note in FRUS 64-68, XXXIV, S. 314ff. 
Abwendung des Bruchs zwischen OPEC und den Konzernen beigetragen hat. ${ }^{236}$ Letztlich war es aber die von Mohammad Rezā selbst durchgesetzte Rückkehr zur zurückhaltenden Verhandlungsposition, welche die OPEC-Front dieses Mal noch aufbrach. Der Kurswechsel des Schahs kostete Ruhāni seinen Posten als Generalsekretär der OPEC und führte zu zusätzlichen Belastungen im Verhältnis zu den anderen nahöstlichen Staaten, insbesondere Iraks, wenngleich sich auch der befürchtete Ausschluss Irans aus der OPEC nicht bewahrheitete. $^{237}$ Vorerst aber konnte sich Iran ein weiteres Mal gegenüber den USA als verantwortungsbewusster Partner in Ölfragen gerieren. ${ }^{238}$

Nachdem die OPEC im November 1964 ihre gemeinsame Position auch formal aufgegeben hatte - eine direkte Konsequenz zuvorderst der iranischen Haltung -, war diese Krise abgewendet. Iran selbst schloss Anfang 1965 eine Kompromissvereinbarung mit dem Konsortium, die allerdings nur zu begrenzten Mehreinnahmen führte. ${ }^{239}$ Das freundschaftliche Verhältnis zwischen Ölkonsortium und iranischer Regierung - seit 1954 ein Stützpfeiler der Beziehungen - verschlechterte sich jedoch im Kontext der Ablösung des amerikanischen Militärmonopols und der iranischen Aufrüstungspläne. Die Tatsache, dass die Wende in der iranischen Ölpolitik zeitlich der unabhängigeren Außenpolitik und den Eigenständigkeitsbestrebungen im Militärsektor und auf anderen Feldern nachfolgte, war wohl gleichermaßen auf Zweifel Mohammad Rezās bezüglich der Durchsetzbarkeit weitreichender Forderungen und der negativen historischen Erfahrungen einer Konfrontationspolitik in der Ölfrage geschuldet. ${ }^{240}$ Angesichts der bisherigen Erfolge der Unabhängigkeitsbestrebungen und des immer größer werdenden Finanzbedarfs war es allerdings nur eine Frage der Zeit, wann Iran in Öl-Angelegenheiten einen ähnlichen Aktivismus an den Tag legen würde.

Anfang 1966, konfrontiert mit fallenden Förderquoten des Konsortiums, kündigte Mohammad Rezā gegenüber Meyer einen bevorstehenden Wandel in der „Philosophie“ der eigenen Ölpolitik an. ${ }^{241}$ Im April warnte er erneut, der Anstieg der Förderung in Iran, gerade im

\footnotetext{
${ }^{236}$ Vgl. Talbot Memo, 16.12.63, FRUS 61-63, XVIII, S. 841ff.; der Schah hatte Holmes noch im Februar 1963 versichert, dass ,,[...] Iran would never embark on a new adventure in this field as a bitter lesson had been learned at the time of Mosadeq. "; Iran werde eher die OPEC verlassen als mit dem Konsortium zu brechen. Vgl. State \#583, 7.2.63; Tehran \#A-501, 7.2.63, PET 3 IRAN, CF 63.

${ }^{237}$ Noch im August 1963 hatte der Schah unmissverständlich geäußert, Iran werde der OPEC-Position treu bleiben. Offenbar war es vor allem britischer Druck auf den Schah, der Iran zum Einlenken bewog. Vgl. Tehran \#A-121, 22.8.63, PET 17-1 IRAN, CF 63; MemCon, 8.6.64, FRUS 64-68, XXII, S. 79f.; Jidda \#973, 12.4.64, FRUS 64-68, XXXIV, S. 321-4; von amerikanischer Seite war ein Verbleib Irans in der OPEC erwünscht. Vgl. Position Paper, ,OPEC‘, o.D. [Januar 1964], ,U.S.-U.K. Talks-25-30 Jan. 1964', Lot 67 D 495, G/PM, Office of Operations, Subject Files, 1962-1966, Box 3.

${ }^{238}$ Die ,pro-westliche“ Ölpolitik Teherans basierte offenbar auch auf der Hoffnung, eine Konfrontation zwischen den arabischen Erzeugerländern und den Ölgesellschaften würde letzten Endes zu einer Erhöhung der iranischen Produktion und Mehreinnahmen für Teheran führen. Vgl. Memo for the Files, 4.5.64, ,POL - Iran/Arab States (General) IRAN 1964‘, Lot 69 D 178, NEA/IRAN: 1964-66, Box 5; zur ,,pivotal role“" Irans in der Zeit aus Sicht westlicher Ölinteressen vgl. BAMBERG, History of the British Petroleum Company: 3, S. 171-84.

${ }^{239}$ Vgl. Cameron Memo, 6.1.65, FRUS 64-68, XXII, S. $120 \mathrm{f}$.

240 „Prime Minister Alam of Iran had told the British Ambassador that in view of the then OPEC impasse, it would not be too difficult to resurrect the Mossadeq spirit in Iran."; MemCon, 29.1.64, FRUS 64-68, XXXIV, S. 318.

${ }^{241}$ Kermit Roosevelt berichtete zeitgleich ähnliche Absichten nach einer seiner zahlreichen Audienzen beim Schah. Vgl. Tehran \#1041, 20.1.66, PET 6 IRAN, CF 64-66.
} 
Vergleich zu den Emiraten auf der anderen Seite des Golfs, sei inakzeptabel. ${ }^{242}$ Für die Iraner war die Aussicht auf eine Produktionssteigerung im laufenden Jahr über 9-11\% nicht ausreichend. Sie forderten $17 \%$ Steigerung jeweils für die beiden kommenden Jahre und im Falle der Nichterfüllung die Zahlung der ausgebliebenen Einnahmen als Vorauszahlung. ${ }^{243}$ Wenngleich die Johnson-Administration beteuerte, die US-Ölkonzerne innerhalb des Konsortiums seien regierungsunabhängig und die amerikanische Gesetzgebung untersage jede Einflussnahme, wurde diesem Argument jedoch keinen Glauben geschenkt. Der ausufernde Streit zwischen Iran und dem Konsortium wurde vielmehr auch zu einer Belastung der zwischenstaatlichen Beziehungen. Angesichts der drohenden Konfrontation zwischen der iranischen Regierung und dem Konsortium führten die USA und Großbritannien Konsultationen in Washington, um für diesen Fall vorbereitet zu sein. ${ }^{244}$ Allgemein befürchtete man, der Schah könnte seine nationalistische Außenpolitik nun auch auf das Feld der Ölpolitik übertragen, mit der Absicht „to out-Mossadeq Mossadeq“. ${ }^{245}$ Mögliche Vergeltungsmaßnahmen gegen das Konsortium waren z.B. eine unilaterale Reduktion des Konzessionsgebiets nach irakischem Vorbild oder sogar die Aufkündigung des Abkommens von 1954. Die USA und Großbritannien wirkten sowohl auf die Ölgesellschaften und die iranische Regierung ein und mahnten zur Zurückhaltung. ${ }^{246}$ Wieder einmal war es Averell Harriman, inzwischen fast ein Vierteljahrhundert mit Mohammad Rezā vertraut, der als Schlichter auftrat. Harriman warnte den Schah mit Nachdruck vor einer Wiederholung der „Tragödie von 1951“, auf die damalige Ölkrise und ihre schwerwiegenden Folgen verweisend, und schien beim Schah durchaus Eindruck zu hinterlassen. ${ }^{247}$ Im Dezember 1966 kam es dann

\footnotetext{
${ }^{242}$ Vgl. Tehran \#1370, 12.4.66, FR US 64-68, XXII, S. 230f.; die Botschaft analysierte: „[...] the Shah and his advisers have come to the conclusion that the easiest way to finance Iran's development and defense programs would be to force the Consortium to produce and export more crude oil."; Tehran \#A-649, 26.3.66, PET 6 IRAN, FN 16, CF 64-66.

${ }^{243}$ Vgl. Bracken Memo, 7.3.66, PET 6 US-IRAN, ebd.; Hughes Memo [INR RNA-38], 3.6.66; Hare Memo, 25.10.66, FRUS 64-68, XXII, S. 261f., 324ff.; gegenüber Meyer begründete der Schah seine Forderung mit der Notwendigkeit, Wachstumsraten der Gesamtwirtschaft von wenigstens $8-10 \%$ in den kommenden Jahren erreichen zu müssen. Vgl. Tehran \#1860, 25.10.66, FRUS 64-68, XXXIV, S. 349ff.; die Beschwerde über den langsameren Anstieg der iranischen Förderung gegenüber der saudischen war im Übrigen berechtigt - die Botschaft machte die britischen, niederländischen und französischen Konsortiumsmitglieder dafür verantwortlich. Vgl. Wriggins Memo, 8.11.66, ,Wriggins Memos, 1966', NSF, Name File, Box 8, LBJL; State \#1053, 20.4.66, FN 16, CF 6466; vgl. BAMBERG, History of the British Petroleum Company: 3, S. 174; auf iranischer Seite vermuteten manche Beobachter einen Zusammenhang zwischen der Verweigerung größerer Öleinnahmen und iranischen Forderungen nach rascherer Aufrüstung. Vgl. Information Bulletin N ${ }^{\circ} 533$, 26.7.66, FO 248/1628, TNA:PRO.

${ }^{244}$ Vgl. State \#75441, 28.10.66; MemCon, 3.11.6, FRUS 64-68, XXXIV, S. 352ff., 354-8.

245 Ebd., S. 357; wenig überraschend bewerteten die Konsortiumsmitglieder die iranischen Forderungen als unannehmbar. Vgl. State \#79406, 4.11.66, ebd., S. 359ff.; die Referenzierung auf Mosaddeg lässt sich immer wieder finden. So warnte der Schah Ende 1967, er sei zum Kampf mit dem Konsortium bereit: ,This time it would not be with a Mosadeq but with a united Iran behind Shah himself.“; Tehran \#2702, 29.12.67, ebd., S. 395.

${ }^{246}$ Vgl. Tehran \#1932/\#2032, 31.10./7.11.66, PET 6 IRAN, CF 64-66; vgl. BILL, Eagle and Lion, S. 170; eine Vereinbarung mit der französischen staatlichen Ölgesellschaft Entreprise de Recherches et d'Activité PetroliersERAP, einem Vorläufer der späteren Elf Aquitaine, über die Ausbeutung eines neuen Konzessionsgebiets illustrierte die mächtiger werdende Verhandlungsposition der Iraner. Im Rahmen dieser Vereinbarung agierten die Franzosen ausschließlich als Vertragsnehmer, das geförderte Öl blieb formal im Besitz der NIOC. Der Deal implizierte eine 75:25-Verteilung der Profite zugunsten der NIOC. Zu weitreichend die Schlussfolgerungen in FERRIER, Iranian Oil Industry, S. $116 \mathrm{ff}$.

${ }^{247}$ Vgl. Tehran \#1964, 2.11.66, FRUS 64-68, XXII, S. 326-30; ed. note, ebd., S. 206; Incoming \#10478, 11.11.66, ,Manila Summit Conference 9 Post Trip Iran', Box 552, Harriman Papers, LOC; im Rückblick wertete man den Harriman-Besuch vom November 1966 als wichtigen Faktor in der Beilegung des Konflikts mit dem Konsortium und als Beispiel für die eigene Rolle als ,,friend“ zwischen den Parteien. Vgl. Administrative History: Iran, S. 19; eine ähnliche Situation wurde Anfang 1968 nach analogem Muster entschärft.
} 
doch zu einem weiteren Kompromiss. Zwar sah man sich seitens des Konsortiums außerstande, auf alle iranischen Forderungen einzugehen. Man sagte aber eine Steigerung der Ölförderung für die kommenden zwei Jahre in etwa analog zum laufenden Jahr zu. Für 1966 rechnete man nun mit einer Steigerung von etwa 12\%. Zugleich beschleunigte man den Ausbau der Förderund Exportkapazitäten. Zusätzlich gab das Konsortium freiwillig etwa $25 \%$ des Konzessionsgebiets auf Basis der Vereinbarung von 1954 auf, was die iranische Regierung in die Position versetzte, neue Konzessionen an Interessenten zu vergeben, sehr wahrscheinlich zu vorteilhafteren Konditionen. Zudem gestand man der NIOC nicht unerhebliche Mengen an gefördertem Öl zu, um es selbst in Osteuropa zu vermarkten bzw. für Barter-Geschäfte einzusetzen, eine aus Sicht der Ölkonzerne alles andere als wünschenswerte Präzedenz. ${ }^{248}$ Insgesamt aber bewertete die Botschaft das ausgehandelte Offtake Agreement vom Dezember 1966 als eine vernünftige Lösung, ,[...] which does not appear to us to set precedents which might be prejudicial to the interests of other producing countries. "249 Zwar zeichneten sich in der zweiten Hälfte der 1960er die zukünftigen Zusammenstöße in der Ölfrage bereits ab, zu diesem Zeitpunkt aber war die Kompromissbereitschaft auf beiden Seiten noch ausreichend, um eine Konfrontation wie dann in den 1970er Jahren vorerst zu vermeiden. ${ }^{250}$ Insgesamt aber führte die Beteiligung amerikanischer Ölgesellschaften am iranischen Konsortium nicht zu verstärktem Einfluss, wie es die politische Folklore zeitgenössisch wie gegenwärtig gerne behauptete bzw. behauptet, sondern hatte immer das Potential, die bilateralen Beziehungen zu belasten.

Dagegen blieb die amerikanisch-iranische Zusammenarbeit auf dem Feld der inneren Sicherheit eine Konstante. Die Zusammenstöße von 1963 hatten zu einer Intensivierung der Kooperation geführt. Angesichts der schweren Unruhen in Teheran hatte ARMISH-MAAG gemeinsam mit der iranischen Armeeführung die Verlegung gepanzerter Einheiten in die Nähe der Hauptstadt organisiert. Zudem strebte man die Verwendung gerade gelieferter amerikanischer M113Transportpanzer ,for use in Tehran“ an. ${ }^{251}$ In einem Bericht an die Special Group (CI) rühmte man die eigene Rolle dabei, eine Wiederkehr von Unruhen im Folgejahr mittels Abschreckung verhindert zu haben. Zivilhilfe auf dem Land wurde parallel ausgeweitet - zugleich setzte man

\footnotetext{
${ }^{248}$ Der NIOC wurden 20 Millionen Tonnen Rohöl für den eigenverantwortlichen Export nach Osteuropa für den Zeitraum von 1967 bis 1971 zu reduzierten Preisen zugesprochen. Vgl. Tehran \#2278/\#2473, 25.11./ 10.12.66; Tehran \#A-340, 24.12.66, PET 6 IRAN; nachdem man von einer geheimen Absprache der verschiedenen Ölgesellschaften innerhalb des Konsortiums erfahren hatte, die es einer Minderheit erlaubte, die Gesamtförderung des Konsortiums zu beschränken, entschloss man sich im State Department, diese Information vorerst geheim zu halten, um weitere Auseinandersetzungen mit der iranischen Regierung zu vermeiden. Vgl. Solomon Memo, 16.12.66, PET 6 IRAN, CF 64-66; tatsächlich waren die Iraner über diese Absprache seit Langem im Bilde. Vgl. Tehran \#3441, 23.2.68, FRUS 64-68, XXXIV, S. 398f.; vgl. FERRIER, Iranian Oil Industry, S. 118.

${ }^{249}$ Tehran \#A-340, vorherig. Anm.; tatsächlich erhöhte sich die iranische Förderung aufgrund des arabischen Ölembargos im Anschluss an den Juni-Krieg von 1967 um 17\% im Vergleich zu 1966. Wenig überraschend forderten die Iraner umgehend den Ausbau der iranischen Förderung auf 4 Millionen barrels/day bis 1970 statt der in der Vereinbarung vom Dezember 1966 vorgesehenen 2,9 Millionen b/d. Vgl. Solomon Memo, 12.10.67, FRUS 64-68, XXXIV, S. 387ff.; Solomon Memo, 11.12.67; Tehran \#2702, 29.12.67, FRUS 64-68, XXII, S. 451ff., $457 \mathrm{ff}$. ${ }^{250}$ Die britische Regierung sah den versöhnlichen Ausgang der Krise als einen weiteren Beleg für die Vorteile enger anglo-amerikanischer Kooperation in Ölfragen. Vgl. MemCon, 3.3.67, FRUS 64-68, XXXIV, S. $373 \mathrm{ff}$.

${ }^{251}$ Vgl. Eckhardt Memo, 2.12.63; Gildardt Memo, 11.11.63, ,DEF 19-2 [...]64‘, Lot 69 D 178, NEA/IRN: 196466, Box 5; Jernegan Memo, 2.3.64, FRUS 64-68, XXII, S. 13-8.
} 
nun die älteren Pläne für den Aufbau einer Sondereinheit innerhalb der Gendarmerie konsequent um. Neue Ausrüstung verbesserte die Effizienz der nationalen Polizei, wenngleich die Einheiten in Provinzstädten als weitaus weniger zuverlässig als die Teheraner Polizei eingeschätzt wurden. Zugleich wurden iranische Fallschirmjägerbataillone in Special Forces nach US-Vorbild umgewandelt und damit die Kapazitäten zur Aufstandsbekämpfung erheblich verbessert. Auch das Public Safety-Programm wurde nun auf die ländlichen Gegenden ausgeweitet. Besonders zweifelhaft war die nun gleichermaßen vertiefte Zusammenarbeit mit der fragwürdigen, ultranationalistischen Miliz der National Resistance Organization. ${ }^{252}$ Ohne Frage verbesserte die US-Unterstützung die Effizienz der iranischen Organe erheblich. Im Sommer 1966 bewertete die Botschaft die interne Sicherheitslage in Iran sogar als „exzellent“. ${ }^{253}$ Hauptsorge war inzwischen die Belastung für SAWAK, die sich aus der ansteigenden Präsenz sowjetischer Berater und Techniker in Iran ergab. Laut amerikanischen Angaben hatte der SAWAK nur eine Sollstärke von etwa 3.000 Beschäftigten und verfügte 1964 über ein sehr überschaubares Jahresbudget von \$2,7 Millionen. Die in zeitgenössischen Berichten und teilweise auch in der Forschungsliteratur zu findenden Angaben hinsichtlich einer gewaltigen Organisation, die sämtliche Bereiche des iranischen öffentlichen wie privaten Lebens durchdrungen habe, sind in den Bereich der Legende zu verweisen. Die in den Jahren nach 1963 mit amerikanischer Unterstützung stark verbesserte Nationale Polizei übernahm in der Folge zahlreiche nachrichtendienstliche Aufgaben vom überlasteten SAWAK. ${ }^{254}$

Die iranisch-amerikanische Zusammenarbeit im Feld der inneren Sicherheit wurde allerdings Mitte der Dekade durch die sich nun abzeichnende Einstellung der Entwicklungshilfe in Frage gestellt. Formal war vor allem die Public Safety-Unterstützung Teil des bald beendeten AIDProgramms. Allerdings einigte man sich rasch auf eine Fortsetzung der meisten Programme. ${ }^{255}$

\footnotetext{
${ }^{252}$,Owing in part to improved organization, newly supplied US riot-control equipment, and sharpened intelligence procedures, repetition of the previous year's disturbances was forestalled."; 1963 und 1964 waren mehrfach Mobile Training Teams der US Special Forces in Iran (,with minimum publicity“), um indigene Kräfte auszubilden. Besonders skeptisch wurde die Polizei in Maschhad und Ġom aufgrund des Vorhandenseins tiefer religiöser Gefühle gesehen - entschlossenes Vorgehen gegen Demonstranten sei eher unwahrscheinlich. Vgl. Jernegan Memo, 23.9.64, ,Special [...]17/8/64‘, Box 5; Jernegan Memo, 28.4.65, ,Special [...]6/3/65', Lot 68 D 451, SG (CI) Records, Box 8; Tehran \#A-233, 14.10.63, POL 23 IRAN, CF 63; Tehran \#A-421, 3.2.64, POL 23-1 IRAN; Tehran \#A-430, 5.2.64, DEF 15-2 US; Tehran \#A-410, 1.2.64, POL 23-1 IRAN, CF 64-6; Minutes Meeting Special Group (CI), 20.5.65, FRUS 64-68, XXII, S. $152 \mathrm{ff}$.

${ }^{253}$ Vgl. Tehran \#A-164, 22.8.66, POL 2-3 IRAN, CF 64-66.

${ }^{254}$ Statt periodischen Reports informierte die Botschaft die Special Group (CI) inzwischen per halbjährlichen informellen Briefen des Botschafters, auch dies ein Ausdruck der inzwischen als ruhig eingeschätzten Sicherheitslage. Vgl. Meyer Letter, 19.3.66; Mudd Letter, 24.3.66; Howison Memo, 2.6.66, ,POL 23-1 [...]66', Lot 70 D 330, NEA/IRN: 1964-66, Box 18; zum SAWAK vgl. National Policy Paper: Iran, Part II, 2.2.67, , Near and Middle East 1965-66', Lot 72 D 139, S/P Records, Subject and Country: 1965-69, Box 315; Harriman Memo, 7.4.66, ,Ball, George‘, Box 434, Harriman Papers, LOC; damit sind die späteren Angaben des gestürzten Schahs über den vergleichsweise kleinen Umfang des SAWAKs wohl als korrekt einzuschätzen. Vgl. PAHLAVI, Answer to History, S. 156f.; vgl. AMUZEGAR, Dynamics, S. 74f.; als Beispiel für die zeitgenösschen Übertreibungen vgl. GRAHAM, Illusion of Power, S. 146, der dagegen von 40.-60.000 Mitarbeitern spricht.

${ }_{255}$ AID verwies früh auf die Absicht, sämtliche Programme bis spätestens Ende des Jahrzehnts einzustellen. Vgl. Towsley Memo, 28.2.64, ,POL 23-1-a [...]64‘, Lot 69 D 178, NEA/IRN: 1964-66, Box 4; neben der Stärkung der iranischen Fähigkeiten zur Aufstandsbekämpfung hatte das Polizeiprogramm noch andere Vorteile: ,the United States training mission has provided the United States with important political and other advantages in a vital part of Iran's power structure."; Howison Memo, 28.7.65; allerdings gab es auch Bedenken, dass die Zusammenarbeit mit iranischen Stellen im Kontext der Aufstandsbekämpfung den Aufbau von ,rival centers of power“ fördere, wie ein Kommentar von GTI in Reaktion auf eine INR-Studie über die US-Unterstützung für iranische Sicherheitskräfte konstatierte. Die Studie selbst ist in den Akten nicht vorhanden, behandelt aber offenbar
} 
Das Weiterbestehen der US-Unterstützung für die Sicherheitsorgane war somit auch ein zentraler Punkt im unten analysierten National Policy Paper für Iran vom Februar 1967. ${ }^{256}$

Die Programme in Iran wurden nur indirekt durch die administrativen Reorganisationen innerhalb der Johnson-Administration in Bezug auf das Counterinsurgency-Feld beeinflusst. Die Euphorie der Kennedy-Phase bezüglich Theorien und Methoden der Aufstandsbekämpfung war merklich verflogen, wenngleich die zunehmende Eskalation des Vietnamkriegs bald zu einem Wiederaufleben führen sollte. ${ }^{257}$ Robert Komer und Harold Saunders drängten 1965 auf eine Neuausrichtung gerade der Special Group (CI) mit Blick auf Fragen ,politischer Entwicklung“ bzw. präventiver Aspekte von Aufstandsbekämpfung. ${ }^{258}$ Im Herbst hatte Präsident Johnson den ehemaligen JCS-Stabschef Maxwell Taylor mit der Begutachtung der amerikanischen Aktivitäten in dem Bereich beauftragt. Teilweise aufbauend auf Taylors Vorschlägen wurde die Special Group (CI) im März 1966 aufgelöst und das Gros ihrer bisherigen Kompetenzen auf die neu gegründete Senior Interdepartmental Group (SIG) und die untergeordneten Interdepartmental Regional Groups (IRG) für die jeweiligen geographischen Regionen übertragen. ${ }^{259}$ Der bisherige CIDP für Iran blieb in Kraft, wobei die Rückübertragung der Kompetenzen in die Obhut des State Department zu einem spürbaren Nachlassen des Interesses an den Programmen führte. ${ }^{260}$ Gleichzeitig diskutierte man Neufassungen der nun als weitgehend überholt geltenden OIDP vom September 1962. Allerdings gelang es auch in den Neufassungen nicht, den Grundwiderspruch zwischen Unterstützung der Sicherheitsorgane in den Zielgesellschaften und damit der Ermöglichung effizienter Repression der Bevölkerungen bei gleichzeitigem Wunsch nach Aufbau nachhaltiger politisch-integrativer Institutionen und der Entstehung stabiler pro-westlicher

retrospektiv die US-Beteiligung am Aufbau von SAWAK, and its former director“. Vgl. Thomas Memo, 2.4.64, ,POL 23 [...]64“, Lot 69 D 178, NEA/IRN: 1964-66, Box 4; weltweit hatten 33 AID-Progamme einen Public Safety-Bestandteil. Vgl. Public Safety Study, 30.8.65, ohne Folder, Lot 75 D 175, NEA/NR: Records Relating to Military Affairs, 1962-70, Box 3; die ohnehin von Anfang an umstrittene Eingliederung der Public SafetyProgramme in AID verkomplizierte die Politikformulierung laut deren Leiter Byron Engle erheblich. Vgl. Saunders Memo, 14.4.65, ,Counter-Insurgency, Special Group, 1964-1965-1966`, NSF, FRWK, Box 15, LBJL.

${ }^{256}$ Vgl. National Policy Paper: Iran, 2.2.67, FRUS 64-68, XXII, hier S. 350f.; AID Administrator Bell hatte die Special Group (CI) bereits im Mai 1965 informiert, das Auslaufen der amerikanischen Hilfsprogramme für Iran müsse nicht zwangsläufig auch das Counterinsurgency-Programm mit einbeziehen. Vgl. Minutes Meeting Special Group (CI), 20.5.65, ebd., S. 152ff.

${ }^{257}$ Rostows umfassendes BNSP-Projekt hatte im Nachgang eine Bestandsaufnahme der Umsetzung der OIDP vom September 1962 ausgelöst. Vgl. Kitchen Memo, 12.3.64, FRUS 64-68, X, S. 49-55.

${ }_{258}$ Saunders bezog sich in mehreren Memoranden auf das Desiderat im Bereich ,politischer Entwicklung“ im Gegensatz zu ,ökonomischer Entwicklung“, sowohl innerhalb der Regierung als auch in der akademischen Welt: „,we still haven't scratched the surface in figuring out what we can do to encourage political institutions that will, over the long haul, take the steam out of insurgency."; Saunders merkte zudem an, dass die Special Group (CI) nicht ,this President's blessing“" habe: „Now everyone thinks LBJ doesn't even know the Group exists.“; Saunders Memo, 13.4.66, ,Counter-[...]-1966“, NSF, FRWK, Box 15, LBJL; Komer Memo, 17.2.65, FRUS 64-68, XXXIII, S. 54; vgl. auch McCone Memo, 18.3.65, ebd., S. 67f.

${ }_{259}$ Vgl. oben S. 423f.; Taylor hatte u.a. eine Neuaufstellung der Special Group (CI) unter der Bezeichnung Overseas Operations Group empfohlen Vgl. Taylor Letter, 17.1.66, encl. to Bundy Memo, 19.1.66, ebd., S. 11120; die Reorganisation wurde umgesetzt mit Johnson Memo [NSAM N³41], 2.3.66, ebd., S. 128ff.; die exakte Aufteilung der Kompetenzen in Record of Agreements and Decisions of the SIG Meeting, 26.7.66, ebd., S. 1738; siehe auch Harriman Letter, 7.3.66, ,Ball, George‘, Box 434, Harriman Papers, LOC; JOHNSON, Right Hand, S. 398-401; zur Auswirkung auf das iranische Programm vgl. Sober Memos, 29.3./7.6.66, ,IRG/NEA Miscellaneous Material'; Report ,U.S. Internal Defense Programs in the Near East and South Asia', 20.6.66, encl. to Sober Memo, 20.6.66, ,IRG/NEA', Lot 68 D 117, ASS/NEA: Subject Files 1965-66, Box 5.

${ }^{260}$ Vgl. Saunders Memo, 14.7.66, ,State, Dept. [...](IRG) [2 of 2]‘, NSF, Agency File, Box 63, LBJL. 
Orientierungen aufzulösen. ${ }^{261} 1968$ schließlich machten sich die Auswirkungen des VietnamDesasters auch in diesem Bereich bemerkbar. Die SIG verabschiedete im Mai eine neue Richtlinie zur Foreign Internal Defense Policy der USA, welche die alte OIDP ersetzte. Gänzlich anders als ihre Vorgänger akzeptierte die neue Richtlinie nun die Existenz gewaltsamrevolutionärer Prozesse in sich entwickelnden Gesellschaften, die nicht zwangsläufig im Gegensatz zu amerikanischen Interessen stünden. ${ }^{262}$ Eine Direktive an sämtliche diplomatische US-Vertretungen ging sogar noch weiter und sprach davon, dass nun das Konzept ,[...] of the United States as the world's anti-communist gendarme is rejected. “263

Der Machtverlust der USA in Kombination mit der wachsenden Bedeutung des gestärkten Iran in den strategischen Konzepten machten ab Mitte der 1960er Jahre eine größere Rücksichtnahme auf die Empfindlichkeiten Mohammad Rezās notwendig. ${ }^{264}$ Der verstärkte Druck auf die oppositionellen iranischen Studenten in den USA wurde bereits geschildert. Angesichts des fortgesetzten Misstrauens des Schahs gegen jeden Kontakt zu nicht-loyalen Iranern bzw. der Opposition gestaltete sich die Arbeit der Botschaft zunehmend schwierig. Man war weiterhin um die Aufrechterhaltung der Kontakte zu den Überbleibseln der Nationalen Front, insbesondere zur jüngeren gemäßigten Fraktion, bemüht. So unterstützte die Botschaft bei aller sonstigen Willfährigkeit gegenüber den Wünschen des Schahs und gegen interne Widerstände z.B. die Erteilung eines Fulbright-Stipendiums an den jungen Oppositionellen Hosejn Mahdawi, zudem ein Mitglied der Nationalen Front. ${ }^{265}$ Zugleich hatte die merklich zunehmende Identifikation der Botschaft mit dem Schah zur Folge, dass kritische Berichterstattung über die Lage in Iran weiter abnahm. William Polk hatte diese Tendenz ja bereits Ende 1963 kritisiert. Dem Politikwissenschaftler und Irankenner James Bill zufolge waren es insbesondere der Botschafter Armin Meyer und der Political Counselor in der Botschaft, Martin F. Herz, die kritische Berichterstattung über das Regime unterbanden und

${ }^{261}$ „Subversive Aggression“ wurde zwischenzeitlich definiert als von außen gesteuerter Umsturzversuch, also weiterhin nicht ausschließlich auf kommunistisch gesteuerte Subversion begrenzt. Zugleich aber stellte man fest: „Purely indigenous insurgency, free of foreign direction or support, is not subversive aggression."; letztlich, so macht der Gesamtzusammenhang klar, entschied die strategische Bedeutung des Lands darüber, ob die USA Hilfestellung leisten würden. Siehe State Department Draft Paper, o.D. [Januar 1967], FRUS 64-6, XXXIII, S. 229 .

${ }^{262}$,In any case, it does not seem possible entirely to deprive people of the use of force for purposes of social change, and action which is too repressive may worsen the problem by forcing the pressures for alterations in institutions outside legal or acceptable channels and into subversive forms."; SIG Paper, ,United States Policy on Internal Defense in Selected Foreign Countries“, o.D. [Mai 1968], FRUS 64-68, X, S. 686-93, hier 688; vgl. auch Katzenbach Memo, 10.6.68, ebd., S. 693ff.; Farley Memo, 19.4.68, encl. to Hartman Memo, 26.4.68, ,SIG/MEMO $\# 61-4 / 26 / 68[\ldots]^{\prime}$, Lot 70 D 263, SIG Files, 1966-1969, Box 2.

${ }^{263}$ State \#CA-8663, 13.6.68, ,SIG Agenda \#34', ebd., Box 2; die Botschaft in Teheran betätigte in ihrer Antwort ein weiteres Mal, die Unterstützungsleistungen im Rahmen der Armee- und Gendarmeriemissionen seien gegenwärtig ausreichend. Wünschenswert sei allenfalls mehr Unterstützung für die nationale Polizei. Vgl. Tehran \#5480, 29.6.68, POL 23 IRAN, CF 67-69.

${ }^{264}$ Während der Debatten über den zweiten \$200-Millionen-Militärkredit hatte der Schah über 'Alam verlauten lassen, es gebe Gerüchte, die USA würden gewisse iranische Persönlichkeiten - wer genau gemeint war blieb unklar - in der Hinterhand halten, ,to upset the situation“ und warnte, er könne womöglich gezwungen sein, den SAWAK auch zur Kontrolle amerikanischer Aktivitäten in Iran einzusetzen. Vgl. Tehran \#A-89, 16.8.66, DEF 19-1 US-IRAN, CF 64-66; vgl auch die Warnung des Schahs, die USA ,[...] should not set in motion political movement in Iran“, offenbar gemünzt auf verdeckte Operationen mit dem Ziel ihn vom Thron zu stürzen. Vgl. Tehran \#80, 7.7.66, FRUS 64-68, XXII, S. 270-4, hier 273.

${ }^{265}$ Vgl. Herz Letter, 20.2.64; Tiger Letter, 12.2.64, ,POL 12-d National Front IRAN 1964', Lot 69 D 225 , NEA/IRN: 1964-66, Box 6. 
Kontakte mit der Opposition zu verhindern suchten. Meyer sei praktisch ein ,public relations officer for the Persian king“" gewesen, so Bill. ${ }^{266}$ Diese Kritik ist sicherlich überzogen, wenngleich korrekt ist, dass Meyer als Botschafter durchaus bisweilen - ähnlich zu vielen anderen US-Botschaftern überall auf der Welt - die Interessen seines Gastlandes mit besonderem Nachdruck zu verteidigen wusste. Nicht zu bestreiten ist, dass man Mitte der 1960er Jahre noch mehr als zuvor Konflikte mit dem Schah zu vermeiden suchte, auch dies Ausdruck der verschobenen Machtverhältnisse. Der zumeist als pro-amerikanisch eingeschätzte SAWAK-Direktor Pākrawān warnte im Sommer 1965 die Botschaft, Kontakte zu iranischen Oppositionellen einzustellen, da das Misstrauen des Schahs gestiegen sei und eine erneute Verschärfung der Repression, allerdings gegen seinen Rat, eingeleitet werde. ${ }^{267}$ Ein offenbar noch zu Zeiten Holmes' in der Botschaft abgefasstes Grundlagenpapier zu den amerikanisch-iranischen Beziehungen definierte als eines der zentralen Politikziele, die Position des Schahs als ,fulcrum of power and a symbol of national unity“ zu erhalten. ${ }^{268}$ Eine solch weitreichende Identifikation mit dem Regime schränkte die eigene Handlungsfähigkeit im Vorhinein stark ein und führte bisweilen zu vorauseilender Selbstzensur.

Enge und persönliche Beziehungen mit der nationalistischen Opposition, wie noch während der 1950er und frühen 1960er Jahre von US-Offiziellen gepflegt, waren mit der neuen Vertrauensbeziehung zum iranischen Herrscher nur mehr schlecht vereinbar. US-Diplomaten wie der bereits mehrfach genannte Bill Miller wurden nicht zuletzt aufgrund ihrer Nähe zur iranischen Opposition ,kaltgestellt“. Im institutionellen Gedächtnis der Botschaft blieb haften, Miller sei „emotionally involved with the National Front“ gewesen. Man habe ihn aufgrund seiner unkontrollierten Kritik am Regime letztlich aus Iran entfernen müssen. ${ }^{269}$ Die Botschaft beschwerte sich auch nach Millers Rückkehr in die USA über seine fortgesetzten Kontakte und

\footnotetext{
${ }^{266}$ Vgl. BILL, Eagle and Lion, S. 173; umso erstaunlicher erscheint es, dass eben dieser Herz später eine Meinungsübersicht aktiver und ehemaliger Diplomaten über die Frage herausgab, wie sich US-Botschaften in autoritären Staaten gegenüber der Opposition verhalten sollten. In Bezug auf sein eigenes Verhalten in Iran führte Herz dabei aus: ,[...] the problem of the American Embassy was not how to establish contacts with the opposition, but how to keep a sense of proportion under the avalanche of derogatory information about the regime received from opposition contacts, and how to preserve a sense of proportion about the importance (or rather, the relative lack of it) of those opposition elements."; HERZ, Contacts With the Opposition, S. vi; im selben Band verteidigt sich auch Meyer. Es habe seines Wissens zu keinem Zeitpunkt eine Anweisung aus Washington gegeben, Kontakte mit Oppositionellen zu vermeiden. Vgl. ebd., S. 26; die Formulierung lässt allerdings offen, ob Meyer selbst als Botschafter entsprechende Kontakte unterbunden habe. Vgl. auch ZoNIS, Majestic Failure, S. 267.

${ }^{267}$ Pākrawān warnte vor Kontakten mit Landbesitzern, Mullahs und ,,people the Shah dislikes“. Auch Treffen mit Amini oder der Nationalen Front seien zu vermeiden. Vgl. MemCon, 4.8.65, ,PPV 1-2 Censorship Iran 1965`, Lot 69 D 426, NEA/IRN: 1964-66, Box 8.

${ }^{268}$ Vgl. Embassy Study ,Policy and Program Summary: Iran', 15.11.64, ,Policy and Program Summary Iran', Lot 69 D 225, NEA/IRN: 1964-66, Box 6; eine Aufdatierung ist Tehran \#A-449, ,U.S. Policy Assessment‘, 30.12.65, POL 2-3 IRAN, CF 64-66.

${ }^{269}$ Ironischerweise finden sich die Hinweise auf Millers Entfernung aus der Botschaft in den erbeuteten Akten im Kontext der Geiselkrise (1979-81). In Band 21 sind Analysen zur Opposition aus der Feder Millers enthalten, was angesichts der zeitlichen Distanz offenkundig für deren Qualität spricht. Eine kritische Fußnote merkte an: ,YYou will note that almost all of the National Fronters and others who were thought to be in opposition to the Shah are solid members of the establishment today."; Miller habe inzwischen ein ,outlet for his crusader zeal“" in seiner Mitarbeit im Church Committee des Senats und in der Untersuchung des Fehlverhaltens der US-Geheimdienste gefunden. Vgl. William Green Miller OHI, 10.2.2003, FAOH; Iran Country Director Eliot Jr. erinnerte sich, Miller habe sich zu sehr mit der iranischen Opposition eingelassen und ,tended to lose sight of some of the positive aspects, from the standpoint of the United States, of the Shah's regime.“; Theodore L. Eliot OHI, 29.7.86, OHIC, S. I-26; vgl. ZONIS, Majestic Failure, S. $266 f$.
} 
die Aktivitäten oppositioneller Iraner, ,[...] who have been brought to the US through the assistance of an American Foreign Service Officer." ${ }^{\text {270 }}$ Miller hatte zudem besagten Hosejn Mahdawi unterstützt und die Veröffentlichung eines kritischen Artikels im renommierten politikorientierten Journal Foreign Affairs ermöglicht. ${ }^{271}$ Der Schah war offenbar äußerst erzürnt über die Veröffentlichung. ${ }^{272}$

Dennoch wäre es falsch, den später in der „Who lost Iran“-Debatte häufig zu vernehmenden Vorwurf kritiklos zu akzeptieren, interne kritische Stimmen hinsichtlich der Politik gegenüber Iran seien systematisch diskriminiert und ausgegrenzt worden. Zum einen gab es tatsächlich wenige solcher Stimmen, begrenzt auf einzelne Offizielle im State Department und in AID. Zum anderen hatten solche Kritiker wie eben Bill Miller zwar in der Tat keinen leichten Stand, doch zeigte sich die grundsätzliche Offenheit für interne Kritik auch daran, dass gerade Miller nach seiner Rückkehr nach Washington gemeinsam mit anderen, eher Schah-kritischen Analysten mit der Abfassung einer INR-Analyse zur politischen Situation in Iran beauftragt wurde, die dann auch größtenteils im späteren National Policy Paper für Iran aufging. ${ }^{273}$ Für Apologeten der Schah-Herrschaft bzw. für Mohammad Rezā selbst nach seinem Sturz waren es gerade Akteure will Bill Miller, Richard Cottam u.a., die fortwährend eine anti-monarchische Grundrichtung der amerikanischen Politik am Leben gehalten und 1978/79 erfolgreich das Ende der US-Unterstützung für den Schah durchgesetzt hätten - eine paranoide aber auch weiterhin gerade bei iranischen Exilanten lebendige Interpretation. ${ }^{274}$ Andererseits ist der von James Bill konstatierte ,negligible impact“ der Schah-kritischen Stimmen auf die tatsächlich verfolgte Politik wiederum nicht zu bestreiten. ${ }^{275}$ In der Retrospektive dominiert die Sichtweise einer fast schon fahrlässigen Vernachlässigung der internen Entwicklungen in Iran und der oppositionellen Gruppen seitens der Botschaft, aber auch der CIA. Eine große Studie der CIA über die politischen Eliten Irans aus dem Jahr 1976 monierte bereits, die letzte umfassende Untersuchung über die iranische Opposition sei 1965 erstellt worden und das eigene Wissen sei daher sehr begrenzt. ${ }^{276}$ Ein ehemalige CIA-Offizier in Iran merkte später den vergleichsweise

\footnotetext{
${ }^{270}$ Herz Letter, 5.4.65, ,POL 12-d National Front Iran 1965`, Lot 69 D 489, NEA/IRAN: 1964-66, Box 11; einem anderen US-Diplomaten, Edward Thomas, wurde die Publikation eines Artikels über die Re-Etablierung der Autokratie in Iran untersagt. Vgl. Franklin Memo, 1.6.66, ,PR 10 Publications 1966', Lot 69 D 491, NEA/IRN: 1964-66, Box 13.

${ }^{271}$ Vgl. MAHDAVY, Coming Crisis; vgl. William Green Miller OHI, 10.2.2003, FAOH; eine Schah-kritische Vorlesung in Harvard des zu Zeiten der Kennedy-Administration häufig als externer Berater für die Iran-Politik herangezogenen Professors Cuyler Young hatte weite Verbreitung in Iran erfahren. Vgl. Tehran \#A-121, 23.8.65, POL 2 IRAN, CF 64-66; der Council on Foreign Relations lehnte 1970 die Veröffentlichung einer von T. Cuyler Young kommissionierten Studie über Iran ab, offenbar aus Sorge über die Reaktion des Schahs. Vgl. BILL, Eagle and Lion, S. 175f.

${ }^{272}$ Vgl. Tehran \#A-235, 4.10.65, POL IRAN-US, CF 64-66.

${ }^{273}$ Vgl. INR Study ,Studies in Political Dynamics Number 13: Iran`, Dezember 1966, DNSA: IR00603; Autoren waren neben Miller zudem Larry Semakis und Archie M. Bolster. Vgl. BILL, Eagle and Lion, S. 176; Theodore Eliot erinnerte sich an diverse Konflikte mit INR, da dort die Überzeugung geherrscht habe, ,that we were overly supportive of the Shah."; Theodore L. Eliot OHI, 29.7.86, OHIC, S. I-53.

${ }^{274}$ Pahlavi, Jamais Résignée, S. 64,70.

275 BILL, Eagle and Lion, S. 176.

${ }^{276}$ CIA Research Study ,Elites and the Distribution of Power in Iran', Februar 1976, Asnād 7, S. 83; die Analyse der inneren Situation in Iran war nicht mehr Priorität für die amerikanischen Geheimdienste: „Near the bottom was the internal political situation."; wichtiger waren in den 1970er Jahren die Aktivitäten der Sowjets in Iran und der Region und bereits hier schon die Nuklearaktivitäten Irans. Der Bericht eines CIA Assessment Committee zur
} 
geringen Umfang der ,political section“ in der Botschaft an - auch die CIA habe dem ,internal reporting“ nur minimale Aufmerksamkeit geschenkt. ${ }^{277}$ Allerdings finden sich auch für die Zeit nach 1965 immer wieder Berichte und Analysen über die Opposition, wenngleich nicht mehr mit der gleichen Frequenz, was der allgemeinen Einschätzung einer stabileren Sicherheitslage geschuldet sein dürfte. ${ }^{278}$ Die in der Literatur - und auch im institutionellen Gedächtnis des State Department - weiterhin lebendige Behauptung, es habe Mitte der 1960er Jahre eine geheime Abmachung mit dem Schah gegeben, wonach die USA jede Sammlung von nachrichtendienstlich relevanten Informationen über die interne Lage einstellen würden, ist nicht zu belegen und ist auch nicht wahrscheinlich. ${ }^{279}$ Zur Verteidigung der Botschaft und der CIA lässt sich zudem anmerken, dass auch die allermeisten journalistischen und akademischen Iranbeobachter bis Mitte der 1970er der Opposition nahezu jede Bedeutung absprachen. Der eigentliche Hauptfaktor hinter dem in der Welt der US-Nachrichtendienste fast schon sprichwörtlichen Intelligence Failure in Iran war eher die Überschätzung der Stabilität des Regimes selbst und der Nachhaltigkeit der Strategie Mohammad Rezās denn eine Fehleinschätzung der Opposition. ${ }^{280}$

Die nachlassende Kompetenz innerhalb der Botschaft und der Nachrichtendienste hinsichtlich der inneren Entwicklungen in Iran zeigte sich gerade bezüglich der religiösen Opposition. Registriert wurden allenfalls die zunehmend anti-westliche Rhetorik der religiösen Rechten, zumal nach der weiter unten analysierten Exilierung Khomeinis. Die Geistlichkeit übernahm nun die traditionelle, stark nationalistische Argumentation hinsichtlich der Kontrolle Irans durch ausländische Mächte: „The problem for the United States with regard to the mullahs becomes clear when we recognize that they are particularly well-positioned in Iranian society to stimulate the latent xenophobia always present here.“ Man müsse sich daher damit abfinden, dass , ,...] some of the inevitable opposition to reforms is bound to rub off on us in the form of criticism of America for supporting these reforms - - even though the criticisms may be

\footnotetext{
Informationslage bez. Iran vom Dezember 1976 ist abgedruckt in Asnäd 8, S. 135-52; zur internen Situationen vertraute man auf die Einschätzungen des in dieser Frage kaum vertrauenswürdigen SAWAK. Letzteres bemängelte man auch ex post facto innerhalb der CIA. Vgl. WOODWARD, Secret Wars, S. 31, 108f.; Senior Review Panel Memo to DCI, 6.1.84, CREST: \#RDP86*006-2; für die Schwierigkeiten oppositioneller Kräfte, sich im repressiven Klima der Zeit überhaupt zu engagieren und für die Anpassung der Protestformen am Beispiel der Arbeiterschaft in der Erdölindustrie siehe JEFROUDI, Labor Activism.

277 DAugherTy, Intelligence Failure, S. 453; der Verlust des institutionellen Gedächtnisses innerhalb der Botschaft machte sich zudem immer stärker bemerkbar. Innerhalb der Botschaft verfügte man über keine Akten über ,political developments in Iran during the Mosadeq days in 1950-53“. Vgl. Armitage Letter, 27.8.64, ,DEF 19-4/a [...]64', Lot 69 D 178, NEA/IRN: 1964-66, Box 5.

${ }^{278}$ Vgl. z.B. die sich noch 1979 in den Akten der Botschaft befindende Studie von 1969 in Tehran \#A-184, 21.4.69, Asnād 20, 1, S. 27-39.

${ }^{279}$ Vgl. DAUGHERTY, Intelligence Failure, S. 457; auch der ehemalige Botschaftsoffizielle George Lambrakis bestreitet, dass es eine solche Abmachung gegeben habe. Vgl. George Lambrakis OHI, 5.6.2002, FAOH; Eliot Jr., der damalige Country Director, bezweifelt die Gerüchte über eine solche Absprache der CIA im Tausch für die Akzeptanz zusätzlicher Abhörstationen. Vgl. Theodore L. Eliot OHI, 29.7.86, OHIC, S. I-26.

${ }_{280}$ Vgl. DONOVAN, Intelligence and the Iranian Revolution; DAUGHERTY, Intelligence Failure; JERVIS, Why Intelligence Fails; vgl. U.S. House of RePresentatives, PERMANENT SELECT CommitTeE On InTELligenCE, SubCOMMITTEE ON EVAlUATION, Intelligence Performance Prior to November 1978; exemplarisch für die zunehmend selbstgefällige Interpretation der „Erfolge“ der US-Politik ist das Memo ,U.S. Accomplishments in Iran, 1961-66“, 15.8.66, ,POL 2 General Reports. Statistics 1966“, Lot 70 D 330, NEA/IRN: 1964-66, Box 18.
} 
couched in different terminology." ${ }^{281}$ Gleichzeitig beruhigte der Autor einer umfassenden Studie über die schiitische Geistlichkeit: „Time is working against the political influence of the Iranian clergy. “282 Martin Herz, der Verfasser dieser Zeilen, war demzufolge überzeugt, jeder Annäherungsversuch der USA an die Geistlichkeit sei vergeblich und ohnehin überflüssig. Allerdings regten sich auch amerikanische Stimmen, die diese Einschätzung nicht teilten. Der US-Konsul in Maschhad warnte, Religion werde auch in der Zukunft weiterhin eine große Rolle in der iranischen Gesellschaft spielen und wies auf Innovationen, in Teilen durchaus fortschrittlich, im religiösen Milieu hin, wie z.B. die lokal zahlreicher werdenden karitativen Einrichtungen: „The United States is faced with the clear choice of ignoring a vital force in Iranian life or of seeking out progressive leaders and institutions hat are prepared to play a positive role. The first course we adopt at our peril; the second offers us opportunity. “283 GTI aber reagierte gereizt auf derlei innovative Ideen. Der Klerus könne durchaus als „,vital force“ angesehen werden, aber ,[...] we should do nothing to reinforce this vitality.“:

We may or may not conclude that, for example, exiling Khomeini was tactically wise; but there will inevitably be occasions when mojtaheds must be combatted. They must not be allowed to stand in the way of the twentieth century, even if they be sincere, charitable and, relative to the stereotype mojtahed, progressive. [...] it seems to me that the 'clergy' as an institution are inherently enemies of the twentieth century, and that we should be careful not to strengthen such an institution gratuitously. ${ }^{284}$

Der umtriebige Bill Miller, zu dieser Zeit bereits in INR im State Department, sekundierte der Sichtweise des US-Konsuls in Maschhad. Es sei fehlgerichtet, den Widerstand von Teilen der klerikalen Hierarchie als etwas rein Religiöses zu interpretieren. Vielmehr sei Khomeinis Gegnerschaft zum Regime symptomatisch für die weitverbreitete Opposition gegen die Regierungspolitik. Im Gegensatz zu den Vorstellungen von Herz', man könne letztlich den widerstrebenden Klerus quasi ,wegmodernisieren“, betonte Miller, aufbauend auf seiner tiefen Kenntnis der iranischen Kultur und Geschichte, die Anpassungsfähigkeit des schiitischen Islams:

As reactionary as the present clergy is, the very nature of religion in Iran is such that it is capable of change and adaptation. Khomeini's opposition represents the reaction of traditional Iranian society. As spokesman for the religious community Khomeini's opposition is, in one sense,

\footnotetext{
${ }^{281}$ Tehran \#A-708, 17.6.64, SOC 12-1 IRAN, CF 64-66.

${ }^{282}$ Ebd.

${ }^{283}$ Meshed \#A-23, 27.2.65, ebd.; der Konsul William Clevenger begründete seinen Vorschlag: „At its worst the clergy clearly merits the epithet 'black reactionary'. I have no sympathy with these elements, and I may know them at closer range than other observers. But the point of my remarks $[\ldots]$ is that they represent a turning of religious energy and funds away from narrow clericalism into more positive and socially responsible lines."; Clevenger Letter, 20.4.65, ,POL 1 Gen. Policy. Background. 1965', Lot 69 D 490, NEA/IRN: 1964-6, Box 12.

${ }^{284}$ Howison Letter, 5.3.65, ,POL 13-6 Religious Groups IRAN 1965‘, Lot 69 D 489, NEA/IRN: 1964-66, Box 11 \{Hervorheb. i. Orig.\}; Howison informierte Herz in der Botschaft, um diese zu mehr ,guidance“ für Clevenger zu ermahnen. Howison verwies allerdings im selben Brief auf seinen eigenen ,ideological bias" in der Frage des Säkularismus. AKHAVI, Religion and Politics, S. 129, zufolge gab es 1965 in Iran etwa 100 höhere Rechtsgelehrte (muğtahid) und 10.000 Mullahs niedrigeren Ranges im Land, organisiert in 20.000 Moscheen.
} 
political protest; more importantly, it indicates the troubled state of Iranian civilization. ${ }^{285}$

Khomeinis Opposition gegen das Regime - und auch vermehrt gegen dessen wichtigsten ausländischen Unterstützer, die USA - fände viel Resonanz in den traditionellen Welten des Basars, in kleinen Städten und auf den Dörfern. Somit würden Schichten und Gebiete in aktiven Widerstand einbezogen, die zuvor nicht gegen die Schah-Herrschaft aktiv gewesen waren. Khomeinis politische Überzeugungen würden, so Miller, von einer „significant mass of Iranians“ geteilt. Darüber hinaus, die Deportation Khomeinis 1964 werde ebenfalls dem Einfluss der USA zugeschrieben: „This new attitude has tarnished our formerly favorable image, poses a threat to our interests in Iran, and will certainly make our task there far more difficult. “286 Eine CIA-Einschätzung nach der Revolution gab Miller im Nachhinein recht. Tatsächlich habe Khomeini bereits seit Längerem bei breiten Teilen der Bevölkerung jenseits seines religiösen Status auch als ,nationalist leader“ Anerkennung gefunden, wohingegen der Schah als „American puppet“ angesehen worden sei. ${ }^{287}$ Andere Warnsignale wurden ignoriert bzw. nachlässig behandelt. Nachdem der SAWAK im Herbst 1965 die im Untergrund operierende „Partei der Islamischen Nationen“ (hezb-e melal-e eslāmi) ausgehoben hatte und 55 Mitglieder vor Gericht gestellt worden waren, registrierte man in GTI das eigene begrenzte Wissen über die religiöse Opposition. Die Botschaft gestand die geringe Zahl direkter Kontakte zum Klerus ein und konstatierte die Schwierigkeit, mit im Untergrund operierenden Gruppen in Kontakt zu kommen. Allerdings war man erstaunt, als im Prozess Details über die Organisation des religiösen Untergrunds (,closely knit and rather efficient“) bekannt wurden. Dennoch dominierte auch im Anschluss die Sichtweise der oppositionellen Geistlichkeit als Übergangsphänomen ohne langfristige Konsequenzen. ${ }^{288}$

\footnotetext{
285 State Paper, o.D. [1965], FRUS 64-68, XXII, S. 122; eine Notiz bestätigt, dass es sich um Ausführungen Bill Millers handelt. Siehe ebd., $n 1$; für eine zeitgenössische akademische Bestandsaufnahme vgl. RAMAZANI, Church and State, S. 26-8.

${ }^{286}$ State Paper, vorherig. Anm., S. 123f.; John Howison schrieb, unerwähnt in der FRUS-Version, auf diese Notiz handschriftlich lapidar „I'm still anti-clerical.“; offenbar versuchten sowjetische Geheimdienstoperationen eben diesen Eindruck zu erwecken. Vgl. Tehran \#A-426, 11.2.65, POL 30-2 IRAN, CF 64-66; in der breiteren Literatur zu den Ursprüngen der Islamischen Revolution finden sich in jüngerer Zeit vermehrt Versuche, die internationale Dimension von Khomeinis theologischen Innovationen angemessen zu berücksichtigen. Offenbar waren antiimperialistische bzw. xenophobe Strömungen nicht nur für den späteren Erfolg der Revolution ausschlaggebend, sondern zugleich ein induktives Element für die Schaffung der revolutionären Weltsicht durch Khomeini und andere zeitgenössische Theoretiker. Vgl. MATIN, Making of Political Islam; RAHIMI, Rise of Shii Ideology.

287 CIA Study, Analysis of NFAC's Performance on Iran's Domestic Crisis, Mid-1977-7 November 1978“, 15.6.79, CREST: \#RDP86*002-5, S. 102; der OSS-Veteran und Orientalist Richard Frye von der Harvard University berichtete nach einem Gespräch mit Khomeini, dieser sei „konservativ“, aber zugleich kein „rabble rouser or fanatic. Khomeini is the direct opposite of Kashani.“; MemCon, 5.11.64, ,POL 30 [...]64“, Lot 69 D 178, NEA/IRN: 1964-66, Box 4.

${ }^{288}$ Zur Untergrundpartei vgl. National Policy Paper: Iran, Part II, 2.2.67, ,Near and Middle East 1965-66', Lot 72 D 139, S/P Records, Subject and Country: 1965-69, Box 315, S. 39; vgl. BILL, Eagle and Lion, S. 162f.; BOROUJERDI, Iranian Intellectuals, S. 83f.; VAHABZADEH, Guerrilla Odyssey, S. 9f.; zur GTI-Reaktion vgl. Herz Letter, 21.2.66, FRUS 64-68, XXII, S. 214f.; die in der FRUS-Version unkenntlich gemachten Namen der Kontaktpersonen finden sich im Originaldokument in den GTI-Akten. Eine CIA-Studie aus dem Jahr 1976 bemerkte trocken, der vornehmlich religiös geprägte Gesellschaftsteil sei nur ,wenig bekannt“. Aus einigen CIABerichten wisse man über stärkeres sowjetisches Interesse am religiösen Feld und Moskaus Kontakten zu Teilen der Geistlichkeit. Vgl. CIA Research Study ,Elites and the Distribution of Power in Iran', Februar 1976, Asnād 7, S. 83, 55; andere Quellen behaupten eine zu den USA analoge Fehleinschätzung der Geistlichkeit durch die sowjetischen Dienste. So habe man Khomeini als pro-amerikanisch eingeschätzt. Vgl. ANDREW/MITROKHIN, Mitrokhin Archive II, S. 180; KUZICHKIN, Inside the KGB, S. 239f.; in einer internen Analyse über die nachrichtendienstlichen Schwächen und Versäumnisse der CIA nach der Revolution findet sich eine interne Kritik an der Vernachlässigung der religiösen Opposition. Der Senior Political Analyst für Iran habe wiederholt, aber
} 
Zu einfach wäre es, diese Phase der Iranpolitik der Johnson-Administration vom Fixpunkt der späteren Revolution rückschauend als Inkubationszeit des späteren Zusammenbruchs sowohl der Schahherrschaft als auch des Endes der vorteilhaften US-Position in Iran zu bewerten. Zwar verfestigten sich in dieser Zeit die bereits unter Kennedy beginnende einseitige Identifikation mit dem Schah-Regime und das Ausblenden, Ignorieren bzw. Fehleinschätzen der weiterhin bestehenden Opposition in Iran, und gerade der religiös motivierten Kräfte darunter. Auch zeichnete sich in dieser Phase bereits die zunehmende Militarisierung und „Versicherheitlichung“ der bilateralen Beziehungen ab, eine Entwicklung, die zwar in erster Linie Resultat iranischen Forderns war, aber letztlich seitens der USA weitgehend kritiklos hingenommen, wenn nicht sogar mitunter gefördert, wurde. In die Johnson-Ära fällt zudem die (erzwungene) Aufgabe der früheren US-Politik, die noch darauf gerichtet gewesen war, den iranischen Ressourceneinsatz auf dem militärischen Feld, wenn schon nicht zu minimieren, so doch wenigstens in eine sinnvolle und nachhaltige Richtung zu lenken. Nunmehr akzeptierte man - teilweise erst nach recht konfrontativ verlaufenden internen Debatten - die grundsätzliche Entscheidungshoheit der Iraner über Struktur und Zielsetzung der eigenen Streitkräfteplanung. Der Kontext des Kalten Kriegs trat angesichts der veränderten Weltlage und des iranisch-sowjetischen Rapprochements dabei in den Hintergrund. Die Militärplanungen Mohammad Rezās waren einseitig auf das regionale Umfeld im Nahen und Mittleren Osten gerichtet: Ziel war die Herbeiführung einer sub-regionalen iranischen Hegemonie rund um die Golfregion, immer mit Blick auf den erwarteten und schließlich 1968 auch angekündigten Rückzug Großbritanniens East of Suez. Angesichts der eigenen Bündnisbeziehung bzw. engen Vertrautheit mit den anderen beiden herausragenden Militärmächten in der Region - der Türkei und Israels - und der geographischen Distanz von Nassers VAR richteten sich die iranischen Rüstungsanstrengungen neben der angedachten zukünftigen Kontrolle des Persischen Golfs in erster Linie gegen den irakischen Nachbarn, der in dieser Zeit die Sowjetunion als Hauptwidersacher und -bedrohung ablöste. Von amerikanischer Seite war man sich bewusst, dass die seitens der Iraner immer wieder vorgebrachte Behauptung einer außerordentlichen Bedrohungslage für die Xuzestān-Provinz vorgeschoben war und in erster Linie als Begründung für die rasante Aufrüstung diente. Angesichts der neuen Unabhängigkeit der Iraner und dem Selbstbewusstsein Mohammad Rezās vermied es die Johnson-Administration aber, die iranischen Militärplanungen ernsthaft in Frage zu stellen. ${ }^{289}$ Mitunter förderte man sogar fehlgeleitete iranische Zielsetzungen, wie z.B. mit

vergeblich, auf diese Schwächen und auf die fehlenden Informationen aufmerksam gemacht. Man habe nichts über die Größe des Netzwerks Khomeinis gewusst und nicht einmal den Inhalt der auf Audiokassetten ins Land geschmuggelten und weit verbreiteten Nachrichten und Predigten gekannt. Vgl. CIA Study, Analysis of NFAC's [... $]^{6}, 15.6 .79$, CREST: \#RDP86*002-5, S. xii.; die Vorstellung einer im Zweifelsfall immer dem Quietismus zuneigenden Geistlichkeit dominierte auch die Einschätzung westlicher Experten im Anschluss an den Sturz des Schahs. Vgl. AmUZEGAR, Dynamics, S. 12f.

${ }^{289}$ Vgl. WARD, Immortal, S. 201-10; militärstrategisch war die Obsession Mohammad Rezās zugunsten des fast ausschließlichen Aufbaus gepanzerter bzw. mechanisierter Einheiten höchst zweifelhaft. CANBY, Military Usefulness, zufolge machte die Kombination aus dieser Schwerpunktsetzung und der gewählten operativen Grundordnung sowohl die Verteidigung gegen einen möglichen sowjetischen Angriff, als auch, aufgrund der 
dem Peterson Report vom März 1966, der eine tatsächliche Bedrohung durch die Nachbarstaaten zu suggerieren schien. Die „Über“-Rüstung Irans begann in dieser Zeit - die einsetzenden Credit Military Sales im Anschluss an das MOU von 1964 können im Nachhinein als der Startpunkt dieses Prozesses identifiziert werden. Die internen Debatten in der JohnsonAdministration demonstrieren dabei ein durchaus greifbares Bewusstsein der potentiellen wirtschaftlichen, sozialen und politischen Folgen von Mohammad Rezās Rüstungsobsession.

Kaum nachweisbar in den Dokumenten ist allerdings eine ernsthafte Rezeption der mittelfristigen Folgen einer militärischen Hegemonie Irans in der Region wie z.B. ein möglicher Rüstungswettlauf mit dem sich (zurecht) bedroht fühlenden Irak und einer Hinwendung Bagdads zur Sowjetunion, um den iranischen Vorsprung zu kompensieren. Kaum findet sich auch die Wahrnehmung langfristig negativer Folgen einer iranischen Dominanz des Persischen Golfes und insbesondere seiner strategischen Zugänge durch Iran aus Sicht der westlichen Allianz. Noch war man glücklich, auch nach dem sich abzeichnenden Ende des Pax Britannica im Golf auf einen ,pro-westlichen“ Partner in der strategisch so bedeutsamen Golfregion bauen zu können - vereinzelt finden sich bereits ab Mitte der Dekade erste Ideen, dem Schah quasi offiziell die Rolle als Stellvertreter westlicher Interessen zu verleihen, eine Vorwegnahme der späteren Nixon-Doktrin. Noch aber war die Erinnerung der Krisenjahre von 1958 bis 1963 zu präsent, um ernsthaft derlei Konzeptionen ins Auge zu fassen. Erst gegen Ende des Jahrzehnts war die Zeit reif für ein Umdenken. ${ }^{290}$

Das zentrale Charakteristikum der bilateralen Beziehungen in dieser Phase ist die Durchsetzung der iranischen Unabhängigkeits- und Souveränitätsansprüche im Anschluss an die Aufgabe des reformistischen Interventionismus. Dieser Prozess zeigte sich insbesondere in der Ablehnung bzw. Suspendierung amerikanischen Einflusses in innenpolitischen Belangen - nach der Einstellung amerikanischer Regulierungsversuche des politischen Systems und der Beseitigung der US-Dominanz in der Wirtschaftsplanung reduzierte Mohammad Rezā die älteste und aus Sicht Washingtons zentrale Abhängigkeitsbeziehung im militärischen Bereich in ihrer Bedeutung und bisherigen Steuerungsfunktion. Die USA hatten sich noch 1962/63 erhofft, über den Umweg des militärischen Felds und gerade mithilfe des Fünfjahres-MAPProgrammes auch in Zukunft dominanten Einfluss auf die iranischen Entscheidungsprozesse ausüben zu können. Mohammad Rezā und die von ihm herangezogenen „neuen Männer“

topographischen Gegebenheiten entlang der Grenze, gegen einen irakischen Einmarsch schwierig. Das Übergewicht an gepanzerten Divisionen sei in erster Linie bei einem eigenen iranischen Einmarsch in die irakischen Ebenen sinnvoll, so Canby. Positiver COTTRELL, Iran's Armed Forces.

${ }^{290}$ LiTTLE, Choosing Sides, S. 184, zufolge habe Johnson seinem Nachfolger einen ,"Three Pillars'-approach“ im Nahen Osten hinterlassen, ,that placed a far higher priority on friendly relations with Israel, Iran, and Saudi Arabia than with Nasser and the Arab radicals."; von der fragwürdigen Qualifizierung Saudi-Arabiens als ,pillar“" abgesehen, erscheint die Einschätzung Littles als ex-post-facto-Rationalisierung. Tatsächlich drehte sich die USPolitik Mitte der 1960er-Jahre bzw. Ende 1966 hin zu einer de facto-Parteinahme gegen den (radikalen) arabischen Nationalismus sowohl in der VAR und im Irak. Die schwerwiegenden strategischen Folgen des Dritten ArabischIsraelischen Kriegs 1967 führten aber rasch zu einer Rückbesinnung auf die strategischen Vorzüge einer BalancePolitik, wenngleich die anvisierte Rückkehr aufgrund der durch den Krieg geschaffenen Tatsachen nicht mehr gelang. 
verweigerten sich aber erfolgreich diesen Einhegungsversuchen und erzwangen gegen anfänglich starken amerikanischen Widerstand die Aufgabe des bisherigen Militärmonopols. ${ }^{291}$ Sicherlich verblieb erheblicher Einfluss der USA aufgrund der nicht zu unterschätzenden Pfadabhängigkeit von Rüstungsentscheidungen und aufgrund des Festhaltens an amerikanischer Hochtechnologie, insbesondere für die zentrale Luftwaffe, und der fortbestehenden Abhängigkeit von Ersatzteilen und Ausbildungsleistungen. Zugleich aber machte sich spiegelbildlich die wachsende strategische Bedeutung des nun regional gestärkten Irans für US-Sicherheitsinteressen und auch die positive Wirkung kräftig anziehender iranischer Einkäufe auf die US-Zahlungsbilanz bemerkbar. Das Phänomen des ,reverse influence“ - der Umkehrung der Abhängigkeiten zwischen Patron und Klienten, nahm ebenfalls hier seinen Anfang. ${ }^{292}$

Jenseits der zu dieser Zeit das bilaterale Verhältnis dominierenden Militärbeziehungen trat die - ohnehin moderate - außenpolitische Neuorientierung in den Hintergrund. Das zentrale Rapprochement mit Moskau und dem Ostblock kann durchaus als Begleitphänomen der Durchsetzung des uneingeschränkten Herrschaftsanspruchs nach innen und der Ablösung des Klientelstatus gewertet werden. Seit Freigabe der Quellen bestätigt sich die bereits von weitsichtigen Zeitgenossen vertretene Einschätzung, dass die US-Administration die Entspannung in den sowjetisch-iranischen Beziehungen im Grundsatz positiv bewertete. Stahlhütten-Geschäft und Waffenkäufe sorgten zwar kurzzeitig für Verstimmung. Das Nachlassen der perzipierten Bedrohung aus dem Norden reduzierte aber die zuvor spürbare Dringlichkeit, die eigenen Militärplanungen für einen möglichen nahöstlichen Ernstfall anzupassen, angesichts der eigenen eskalierenden Verstrickung in Südostasien eine ohnehin nicht realistische Option. Wie in Kapitel VI.3. gezeigt, ermöglichte nicht zuletzt die veränderte Bedrohungslage in der Großregion den Übergang zu einer amerikanischen (militärischen) Offshore-Strategie im Indischen Ozean und im Persischen Golf. Belastend für die Zukunft wirkte sich aber aus, dass es Iran und den arabischen Staaten und den (vorstaatlichen) Entitäten auf der anderen Golfseite nicht gelang, eine Einigung bezüglich der meisten offenen territorialen Fragen und diverser (maritimer) Grenzziehungen zu erzielen. Angesichts des nun allgemein erwarteten britischen Rückzugs in der nahen Zukunft drohten somit neue Konflikte, die wiederum die Stabilität zu gefährden drohten.

\footnotetext{
${ }^{291}$ Andrew Johns sieht den Staatsbesuch des Schahs vom August 1967 als die „culmination of the patron-client relationship that dated back to 1953“; JoHnS, Changing Pattern, S. 64, er sieht die Transformation hin zu mehr Eigenständigkeit erst 1965 beginnen. Die hier vorliegende Untersuchung datiert die Anfänge dieses Prozesses auf 1962/63 und sieht ihn bereits 1966 weitgehend abgeschlossen, wie auch die interne Bestandsaufnahme vom Mai 1966 illustriert. Vgl. oben S. 508, Anm. 175; Johns und McGlinchey erkennen die Bedeutung der Waffenverkäufe auf Kreditbasis, unterschlagen aber beide die zentrale Bedeutung der Auflösung des amerikanischen Militärmonopols. Vgl. Johns, Changing Patterns, S. 89; McGlinchey, Arms Credit Sales, S. 246; die frühen Ansätze iranischer Autonomiebestrebungen korrekt datierend CASTIGLIONI, Start a Revolution, S. 114f.

${ }^{292}$ Vgl. CARR, United States-Iranian Relationship; die Behauptung Carrs, im Regelfall habe Iran die allgemeine Richtung des Verhältnisses vorgegeben, geht allerdings für diesen Untersuchungszeitraum zu weit, mag aber auf die erste Hälfte der 1970er Jahre durchaus zutreffen.
} 
Insgesamt ergibt sich für die Johnson-Zeit ein Bild der bilateralen Beziehungen, das auf intergouvernementaler Ebene durch ein freundschaftliches Verhältnis und - bei allen Eigenständigkeitsbestrebungen der Iraner - auf der Wahrnehmung gemeinsamer und geteilter strategischer Interessen begründet war. Die kurzen „Krisen“-Phasen in den Beziehungen allesamt im Kontext von Aufrüstungswünschen Mohammad Rezās - verschwanden genauso rasch wie sie entstanden waren. Auch zeitigten sie keine längerfristigen negativen Folgen für das gegenseitige Verhältnis. Die von Stephen McGlinchey jüngst vertretene revisionistische Auffassung, die Auseinandersetzungen über die Waffengeschäfte hätten das bilaterale Verhältnis ,[...] extremely rocky after 1964, and critical by mid-1966“ werden lassen, sind auf mangelnde Quellenkenntnis zurückzuführen und in das Reich der Legende zu verweisen. ${ }^{293}$ Eine genauere Betrachtung der kleineren „Krisen“ in dieser Zeit veranschaulicht eher ein auch die Zukunft bestimmendes Phänomen, nämlich den Erfolg der im Grunde von Mohammad Rezā seit seiner Thronbesteigung angestrebten und erhofften besonderen Beziehungen zum jeweiligen Amtsinhaber im Weißen Haus. Was unter Truman, Eisenhower und Kennedy kaum oder nicht möglich war, gelang nun unter Lyndon Johnson. Der persönliche Stil und die vielleicht vorsichtig als reduktionistisch zu kennzeichnende Weltsicht Johnsons spielten der Strategie des iranischen Monarchen hier in die Karten. Ähnlich wie bei Kennedys „Entscheidung“ - eigentlich eher ein über mehrere Wochen laufender inkrementeller Prozess das „Amini-Experiment“ aufzugeben, zeigte sich auch mit der bereits Mitte 1964 dokumentierbaren Tendenz Johnsons, sich im Zweifelsfall immer zugunsten der Rüstungswünsche des Schahs auszusprechen, die Bedeutung des US-Präsidenten als höchster „Entscheider“ im Politikformulierungsprozess. Die Bürokratie verinnerlichte die präsidentiellen Wünsche diesbezüglich und in der Regel setzten sich die Befürworter ansteigender Waffenlieferungen im bürokratischen Prozess bereits im Vorfeld durch - wann immer dennoch eine Entscheidung des Weißen Hauses notwendig wurde, entschied Johnson grundsätzlich im Sinne Mohammad Rezās. ${ }^{294}$ Auch Iran Country Director Eliot Jr. betonte später den Aspekt der engen persönlichen Beziehungen. Häufig von Zeitgenossen geschilderter Ausdruck dieser Innigkeit zwischen beiden Männern waren gemeinsame Gartenspaziergänge, Arm in Arm, während der diversen USA-Besuche des iranischen Monarchen: „They were very, very different men, but they saw the world through very similar eyes. “295 Diese persönliche Bindung überdeckte in der Regel auch Johnsons durchaus vorhandene Prinzipien hinsichtlich guter Regierungsführung und insbesondere wirtschaftlicher und sozialer Reformen. Im Grundsatz plädierte auch Lyndon Johnson für ein Junktim zwischen der Gewährung weiterer Aufrüstung durch die USA und der fortgesetzten Überprüfung der wirtschaftlichen

\footnotetext{
${ }^{293}$ MCGLinCHEY, Arms Credit Sales, S. 230; ähnlich S. 247: ,at times highly-strained period of history between Iran and America"; McGlinchey will die seines Erachtens fehlgeleitete etablierte Sichtweise der Beziehungen in dieser Periode korrigieren; ebd., S. 230; die hier vorliegende Studie bestätigt im Großen und Ganzen die allgemeine Bewertung der bilateralen Beziehungen als im Kern freundschaftlich und entgegenkommend, sicherlich eine zutreffende Interpretation im Vergleich zu den allgegenwärtigen Spannungen in der Phase reformistischer Intervention.

294, ,...] we didn't run into too many bureaucratic obstacles, and when we did they were very easy to override because we knew we had the support of the President."; Theodore L. Eliot, Jr. OHI, 24.4.92, FAOH.

295 Theodore L. Eliot OHI, 29.7.86, OHIC, S. I-52; vg. ebd., S. I-47.
} 
Tragfähigkeit weiterer Zukäufe. Dieses Junktim aber bestand immer nur formell und wurde von den Iranern leicht überwunden, zumal der US-Präsident dem Image Mohammad Rezās als großer Reformer nur allzu gern Glauben schenkte. Repräsentativ für Lyndon Johnsons Wahrnehmung Irans und der Vorgänge dort war sein Ausruf gegenüber David Lilienthal im Juni 1964: „Dave, what is going on in Iran is about the best thing going on anywhere in the world. “'296

In der Retrospektive strebten ehemalige Offizielle der Johnson-Administration danach, die Verantwortung für die unkontrollierte Aufrüstung Irans mit all ihren fatalen wirtschaftlichen und politischen Folgen der Nachfolge-Administration zuzuschreiben. In der Tat zeigte sich die Nixon-Administration bald noch willfähriger gegenüber iranischen Wünschen nach den neuesten Waffensystemen und die Rüstungseinkäufe Irans multiplizierten sich in dieser Zeit um ein Mehrfaches auf ein schwindelerregendes Niveau. Gemessen am Anteil am iranischen Bruttosozialprodukt, ist aber auch schon für die Johnson-Jahre begründet von massiven Rüstungsausgaben zu sprechen. Die häufig vorgebrachte Apologetik, man habe ernsthaft vor den eigenen Verkäufen die wirtschaftliche Gesamtlage und die iranische Zahlungsbilanz geprüft und in die Kalkulation miteinbezogen, deckt sich nicht mit der hier vorliegenden Rekonstruktion. ${ }^{297}$ Nicht übersehen werden darf aber die bei den Zeitgenossen immer gegenwärtige Überzeugung, die Dinge in Iran seien im Begriff sich zum Besseren zu wandeln, ein subjektiver Eindruck, der aber im Vergleich zur jüngeren Vergangenheit durchaus plausibel erschien. Die wirtschaftliche und soziale Lage in Iran verbesserte sich in der Tat rapide zu dieser Zeit, wenngleich kritische Beobachter registrierten, wie einseitig der geschaffene Mehrwert bestimmten Schichten zugute kam. Angesichts eines augenfällig gescheiterten Nation-Building-Experiments in Südostasien ist aber zumindest verständlich, dass man aus amerikanischer Sicht nur allzu erpicht war, mit Iran zumindest ein von Erfolg gekröntes Gegenbeispiel im Nahen und Mittleren Osten vorweisen zu können. Die Identifikation mit der Pahlawi-Monarchie wurde zudem verstärkt durch die nun abgeschlossene Etablierung eines pro-iranischen Netzwerks nahe den amerikanischen Entscheidungsorganen, aber auch in Kongress und Medien, den so genannten Pahlavists. Deren Einflussnahme half nicht nur das Image des iranischen Autokraten in den USA zu verbessern - wobei eine kritische

\footnotetext{
${ }^{296}$ Eintrag vom 16.6.64, in: LiLIENTHAL, Journals: VI, S. 47f; BILL, Eagle and Lion, S. 176ff., sieht Johnsons Unterstützung für die Pahlawi-Autokratie auch in seiner persönlichen Präferenz für und seinen Glauben an die Wirksamkeit gewaltsamer Lösungen begründet. Bill zufolge habe LBJ auch das brutale Vorgehen gegen Demonstranten 1963 gebilligt. Er und seine Berater hätten geglaubt, ,[...] the Shah could not be tough enough"; ebd., S. 156; eine bewusste amerikanische Präferenz zugunsten verstärkter Repression ist zumindest für den iranischen Fall in den Quellen nicht belegbar. Plausibler erscheinen Bills alternative Determinanten der Johnson'schen Iranpolitik: Dankbarkeit für die iranische öffentliche Unterstützung der amerikanischen Interventionen in Vietnam und der Dominikanischen Republik, Parallelität der strategischen Interessen in der Nahostregion und iranische Unterstützung für Israel und neue wirtschaftliche Attraktivität des nun zahlungskräftigen Iran.

${ }^{297}$ Z.b. bei Theodore L. Eliot, Jr. OHI, 24.4.92, FAOH; Armin Meyer porträtierte die US-Politik unter Johnson als ausgewogen. Erst unter Nixon sei die Wende zu ,uncritical support“ erfolgt. Vgl. MEYER, Quiet Diplomacy, S. 146f., 155ff.; nicht haltbar ist die Einschätzung von COHEN, Balancing Interests, S. 286, wonach Johnson die Kennedy-Politik fortgesetzt habe, und versucht habe Waffenkäufe zu begrenzen,,,and to make them contingent on political and economic reform."; uninformiert RuBIN, Good Intentions, S. 116; LiTTLE, Choosing Sides, S. 153, stellt Johnson schablonenhaft als einseitigen Befürworter von Irans Aufrüstung und Unterstützer des Schahs dar.
} 
Gegenbewegung und die oppositionellen Aktivitäten der Auslandsstudenten in den 1970er Jahren erfolgreich ein Gegennarrativ etablieren sollten - und wirkte zudem im Sinne Teherans auf wichtige Entscheidungen wie z.B. die Aufstockung der Rüstungseinkäufe von 1966 ein. Das ,pro-iranische Netzwerk" sollte im Laufe der Jahre zu einem bedeutenden Faktor in den Beziehungen heranwachsen. ${ }^{298}$

Für den zeitgenössischen Beobachter aber musste die US-Position in Iran um das Jahr 1967 und die Entwicklung in der jüngeren Vergangenheit wie eine Bestätigung für die Richtigkeit des eigenen Ansatzes wirken. Iran präsentierte atemberaubende Wachstumszahlen, National- und Per-Kopf-Einkommen stiegen rasant parallel zu den Öleinnahmen. Das Land war nicht mehr auf Entwicklungshilfe angewiesen und ,graduierte“ mit der Einstellung der USWirtschaftshilfe, die sicherheits- und außenpolitische Position schien besser als je zuvor seit Ende des Zweiten Weltkriegs, die Streitkräfte wurden rasch modernisiert und ausgeweitet und das Ansehen des Schahs in der internationalen Politik stieg wahrnehmbar. Die innere Opposition schien dabei geschrumpft und war offenkundig unfähig sich in einer Weise zu organisieren, um die Pahlawi-Herrschaft ernsthaft in Frage zu stellen. Die USA hatten zwar das Gros ihres früheren Einflusses eingebüßt und ihre Mitsprache bei Wirtschafts- und Finanzplanung und in vielen anderen Bereichen verloren. Auch hatte die enge militärische Zusammenarbeit die Hoffnungen, die man ihn sie gesetzt hatte, nicht erfüllt. ${ }^{299}$ Das USMilitärmonopol in Iran war gebrochen, nachdem es über 20 Jahre Bestand gehabt hatte. Dennoch blieben die USA der wichtigste äußere Partner Irans - die Kooperation bei hochsensiblen Waffensystemen wie den F-4-Kampfflugzeugen erschuf neue Abhängigkeiten und sicherte so amerikanischen Einfluss in der Zukunft. Die Streitkräfte spiegelten in Ausrüstung, strategischer Ausrichtung und Organisation das amerikanische Vorbild wider. Viele iranische Offiziere durchliefen Ausbildungsprogramme in den USA und blieben dem früheren Patron in ihrer gesamten Karriere verbunden und häufig wohlgesonnen. Mit Aufbau der neuen Horchstationen wurde die geheimdienstliche Kooperation aufs Neue intensiviert. Selbst der überraschende Tod Mohammad Rezās bzw. seine anderweitige Beseitigung hätten, so das Kalkül, die US-Position nicht nachhaltig gefährdet.

Was die zunehmende Repression in Iran und die immer weiter reichende Dominanz des politischen Systems durch den Monarchen anging, so finden sich in den Quellen nur vereinzelte Gegenpositionen von US-Offiziellen wie William Polk oder William Miller, die vor den langfristigen Folgen einer amerikanischen Parteinahme zugunsten der Autokratie warnten. Im Regelfall - und diese Einschätzung gilt auch für Johnson selbst - waren Skrupel bezüglich der

\footnotetext{
${ }^{298}$ BILL, Eagle and Lion, S. 178, sieht die Pahlavists als einen zentralen Faktor. JoHnS, Changing Patterns, S. 66, 91f., hält innenpolitische Faktoren für maßgeblich, wobei er insbesondere die Rolle des Kongresses und politischer Persönlichkeiten wie Richter Douglas einbringt. Die vorliegende Untersuchung bestätigt diese Einschätzung nicht. Der Wandel hin zu einem positiven Image in der US-Presse wird nachgezeichnet in DORMAN/FARHANG, U.S. Press and Iran, S. 116-30.

${ }^{299}$ CASTIGLIONI, Start a Revolution, S. 125, hingegen behauptet, dass das ,instrument of arms supply eventually emerged as the new, solid cornerstone of the alliance."
} 
US-Politik im Bereich der Menschenrechte kaum auszumachen, zumal man selbst mit der fortgesetzten Unterstützung im Counterinsurgency-Bereich aktiv die Repressionsfähigkeiten des iranischen Staats optimieren half. Die seitens einiger Autoren konstatierte „Wende“ in der amerikanischen Iranpolitik von Kennedy zu Johnson gab es nicht: Bereits Kennedy hatte sich spätestens 1963 mit seinem Glückwunschtelegramm zum Referendum öffentlich auf die Seite des Schahs gestellt. ${ }^{300}$ Nur für wenige Zeitgenossen war das Einsetzen neuer, zuerst nur latenter Entwicklungen wahrnehmbar, die nur etwas mehr als ein Jahrzehnt später sowohl das Ende der Stabilität in Iran wie auch der herausgehobenen amerikanischen Position bewirken sollten. Im Untergrund entstand langsam das Grundgerüst einer neuen Koalition aus traditionellen wie jüngeren nationalistischen Widerstand und der sich seit Anfang der 1960er bildenden religiösen Opposition, die bis Ende der 1970er Jahre eine die Mehrheit der iranischen Bevölkerung umfassende Mobilisierungskraft entfalten sollte. Diese neue oppositionelle Koalition zeichnete sich nicht allein durch Ablehnung jeden Kompromisses mit der verhassten Monarchie aus, sondern zusätzlich durch eine ultra-nationalistische und in Teilen chauvinistische Einstellung gegenüber der Einmischung äußerer Mächte und gerade der USA. Dass die Ablehnung amerikanischen Einflusses und der Bruch der Beziehungen zu den USA - nicht allein der diplomatischen - zu einem Kennzeichen der späteren Revolution werden sollte, hatte ihren Ursprung in den 1960er Jahren. Der zuvor vereinzelt auftretende Anti-Amerikanismus, meist eine Folge des Coups von 1953, wurde in dieser Zeit schrittweise zu einem Massenphänomen und im Anschluss zu einer zentralen Komponente des revolutionären Programms von 1978/79.

\section{4. Souveränität und ihre Folgen: Die Geburt des «amerikanischen Schahs»}

Insgesamt war in der Johnson-Administration Mitte der 1960er Jahre so gut wie keine Besorgnis angesichts des verfolgten Kurses gegenüber Iran und dem autoritären Regime des Schahs zu registrieren. Es gab nur wenige Ausnahmen. Es war wieder William G. Miller, der zweite Sekretär der Botschaft und anerkannte Iranexperte ${ }^{301}$, der seinen Übergang vom mehrjährigen diplomatischen Dienst in Isfahan und Teheran zur nachrichtendienstlichen Analyse in INR im State Department für eine umfassende Bestandsaufnahme der Transformation Irans und der daraus resultierenden Folgen für die amerikanische Position in dem Land nutzte. Bereits im Sommer 1964 verfasste er eine fünfzigseitige Studie mit dem Titel

\footnotetext{
300 BILL, Eagle and Lion, S. 176, spricht von einem ,crossover in Iranian-American relations“ durch LBJs Verschiebung der Politik ,,into decidedly pro-Pahlavi directions“. Bei aller auch hier konstatierten persönlichen Nähe zwischen Johnson und Mohammad Rezā geht diese Feststellung dennoch zu weit. Tatsächlich waren die ersten Amtsjahre Johnsons bezüglich der Politik gegenüber Iran von großer Kontinuität geprägt. Ähnlich CASTIGLIONI, Start a Revolution, S. $115 \mathrm{f}$.

${ }^{301}$ Laut William Polk vom Policy Planning Council sah man Miller in der akademischen Welt als den ,,best informed man in America on Iran“; Polk Letter, 23.4.65, Lot 72 D 139, S/P Records, Subject and Country: 196569 , Box 315.
} 
„The «New Men» and Their Challenge to American Policy in Iran“ “ ${ }^{302}$ In der Studie bestätigte Miller die gängige Einschätzung der Sicherheitslage als zufriedenstellend (,The internal security of Iran has never been better.") und die großen Erfolge im ökonomischen Bereich in den letzten zehn Jahren: Iran bewege sich in Sauseschritten in Richtung Moderne. Zugleich aber registrierte Miller einen grundsätzlichen Widerspruch im sich entwickelnden Iran. Dabei nahm er offenbar Bezug zur bereits erwähnten Analyse von William Polk zu den politischen Effekten von Modernisierung und dem Erscheinen neuer technokratisch-administrativer Schichten. Die eigentlichen Träger der umfassenden Modernisierung, die technokratische und administrative Elite Irans, die von Miller ebenfalls so bezeichneten „Neuen Männer“, stellten denjenigen Personenkreis, der aus politischer Sicht die größte Unzufriedenheit mit der Situation im Lande artikuliere. Die autoritäre Herrschaft des Schahs verhindere eine Teilnahme dieser modern ausgerichteten und zudem bestens ausgebildeten Klasse am politischen Prozess. Im Gegensatz zu weitverbreiteten Vorstellungen sei es zudem diese neue Klasse und eben nicht der Schah, der die neue Stabilität in Iran zu verdanken sei. Somit seien die gängigen Kassandrarufe hinsichtlich eines Abgangs Mohammad Rezās unangebracht - für diesen Fall sei mit einer Fortsetzung des gegenwärtigen Kurses zu rechnen, wenn es denn gelänge, dieser neuen Klasse auch politische Legitimität zuzusichern. Somit laute die herausragende Aufgabe für die amerikanische Iranpolitik, ,[...] how to bring the New Men of ability into the positions of power they are clearly best able to hold without destroying the present stability of the country. “303

Im Kern evozierte Millers Argumentation Gedankengänge, welche der reformistischen Intervention zugrunde gelegen hatten, wenngleich ohne den damals gegebenen Kontext innenpolitischer Krisen und Instabilität in Iran. Zugleich distanzierte er sich vom früheren Vorgehen und dem Aufstellen falscher Alternativen. Statt von einer Wahl zwischen der Stabilität der Monarchie und dem Chaos infolge einer Machtübernahme der nationalistischen Opposition sprach Miller stattdessen von der gebotenen wie dringlichen politischen Versöhnung zwischen zwei modernisierenden und reformistisch eingestellten politischen Kräften, deren Zusammengehen Stabilität und nachhaltige sozio-ökonomische Entwicklung auch für die Zukunft garantieren könnte. Die neue Klasse der Technokraten und Modernisierer sei zwar politisch mit der alten nationalistischen Opposition verbunden und vertrete im Grundsatz deren Gegnerschaft gegen die autoritäre Herrschaft des Hofs. Angesichts der Weißen Revolution, des Aufbegehrens anti-modernistischer Kräfte wie der islamischen Geistlichkeit, und der, durchaus opportunistisch zu interpretierenden eigenen Karrierechancen

\footnotetext{
302 Miller Study, o.D. [1964], ,POL 1 [...]65‘, Lot 69 D 490, NEA/IRN: 1964-66, Box 12 \{im Folgenden: Miller Study\}; in einem späteren Oral History-Interview beschreibt Miller eine unmittelbar nach seiner Ankunft in INR im State Department abgefasste Studie über Political Dynamics in Iran. Die hier analysierte Studie zu den New Men ist in gewisser Weise ein Vorläufer derselben. Vgl. William Green Miller OHI, 10.2.2003, FAOH; vgl. oben S. 530, Anm. 273 und unten S. 545, Anm. 321.

${ }^{303}$ Miller Study, S. 2; Miller ignorierte die Distanz zwischen diesen modernistisch-technokratischen neuen Eliten und der breiten Bevölkerung, insbesondere die Verletzung religiöser und traditioneller Befindlichkeiten infolge der Reformen. Vgl. AzIMI, Quest for Democracy, 228.
} 
im prosperierenden Iran seien sie aber zugleich zur politischen Integration bereit, solange man ihren politischen Sehnsüchten und ihrer Würde durch entsprechende Adaption des politischen Systems entgegenkäme. ${ }^{304}$ Die Betrachtungsweise der internen Situation in Iran als alternativlos sei irreführend: Tatsächlich ließe sich die Integration dieser neuen Klasse auch unter Beibehaltung der Monarchie und ihrer stabilisierenden Wirkung bewerkstelligen. Bedingung aber sei die Abgabe von Macht durch Mohammad Rezā, eine politische Öffnung des Systems und die Einberufung einer dem Parlament verantwortlichen Regierung unter einem starken Premier, im Grunde das Amini-Modell, allerdings mit einer weitaus stärkeren parlamentarischen Komponente. ${ }^{305}$

Es ist wohl nicht falsch, Millers Interpretation als taktisch zu interpretieren. Nach Jahren des diplomatischen Dienstes in Iran und diversen Zusammenstößen mit den überwiegend antireformistisch eingestellten Foreign Service Officers im State Department hatte Miller deren Argumentation eingehend studiert und die Überzeugungskraft ihrer Argumente auf der politischen wie strategischen Ebene erkannt. Statt wie früher aus idealistischer Perspektive zugunsten der Nationalisten in Iran zu argumentieren, deren Machtübernahme nach alter Lesart der Reformisten zur Entstehung eines liberalen und pro-westlichen Irans führen würde, arrangierte sich Miller nun mit der gestärkten politischen Hebelkraft des Schahs. Er plädierte daher für eine Beibehaltung der stabilisierenden Kraft der Monarchie und einer Parlamentarisierung und Demokratisierung des Regimes von innen - selbstverständlich infolge amerikanischer Überzeugungsarbeit und auch diplomatischen wie verdeckten Drucks ${ }^{306}$ mittels der Positionierung der New Men in politische Schlüsselpositionen. Auf diese Weise versuchte Miller, die früheren Gegensätze zu transzendieren: Die USA könnten sowohl ihre strategischen Bedürfnisse nach Stabilität in Iran wie auch ihre idealistischen Wünsche nach guter und demokratischer Regierungsführung in Iran befriedigen. ${ }^{307}$ Ohne Rückkehr zum reformistischen Interventionismus in Iran und bei Beibehaltung der engen Beziehungen zur

\footnotetext{
${ }^{304}$ Entscheidend für Miller war die Transformation der alten nationalistischen Opposition in eine staatstragende technokratisch-administrative Klasse, ein Prozess, den ironischerweise der unter Hausarrest stehende Mosaddeg selbst durch die von ihm maßgeblich betriebene Auflösung der Nationalen Front beschleunigt habe. Ebd., S. 8, 9; in seiner Beschreibung der neuen Klasse aber wird offenbar, dass der politische Kern dieser Gruppe auch für Miller aus den tradierten Führungsfiguren der Front und den Mitgliedern des ehemaligen pro-amerikanischen Netzwerks bestand. Überzeugender war Millers Argumentation, dass die etwa 30.000 Universitätsabsolventen pro Jahr die eigentliche Massenbasis der Opposition ausmachten - diese würden inzwischen den Großteil des Personals in fast allen Ministerien stellen. Vgl. ebd., S. 17f.

${ }^{305}$ Vgl. ebd., S. 15; tatsächlich schlug Miller sogar konkret die Rückkehr Aminis als Premier vor.

${ }^{306}$ In einem späteren Oral History-Interview erwähnte Miller seine bereits damals bestehende Gegnerschaft gegen die herrschende Lesart der inneren Entwicklung in Iran, derzufolge ,economic transformation would lead eventually to political transformation."; William Green Miller OHI, 10.2.2003, FAOH; in der Retrospektive glorifizierte Miller aber selbst ex post facto die Politik der demokratischen Administrationen in Iran. Man habe eine „schizophrene Politik" verfolgt, indem man einerseits resolut demokratische Reformen angemahnt und rechtsstaatliche Prinzipien sowie freie Wahlen eingefordert, andererseits aktiv den repressiven Staatsapparat unterstützt habe. Allerdings habe man im Gegensatz zu Nixon erheblichen Druck zugunsten demokratischer Transformation ausgeübt: ,The shah was under tremendous pressure from us to allow the national democrats to have a role in government for about four years, during the time I was there."; die hier analysierten Quellen zeichnen ein gegensätzliches Bild.

307 Offenbar war Miller in seiner These einer graduellen demokratischen Transformation von innen stark von den politischen Ansichten seines Freundes und Mitgliedes der Nationalen Front, Hosejn Mahdawi, beeinflusst. Vgl. Miller Study, S. $20 \mathrm{ff}$.
} 
Schah-Diktatur, so seine Warnung, würde sich diese neue Elite mittelfristig trotz der gegenwärtig pro-amerikanischen Ausrichtung und der für die New Men typischen Ausbildung in westlichen Staaten, insbesondere in den USA, mittelfristig von der Führungsmacht abwenden und diese als neo-koloniale Fremdherrscher perzipieren. ${ }^{308}$

Im Kern verband Miller seine Wahrnehmung sozialen Wandels im Iran Mitte der 1960er Jahre mit den alten Argumenten zugunsten der Schaffung einer neuen politischen Synthese. Unbestreitbar war, dass die rapide Modernisierung, soziale Transformation und der Ausbau der bestehenden Bürokratien zu einem Anwachsen, ja einer Dominanz, der gebildeten urbanen Mittelschichten in Verwaltung und Privatwirtschaft geführt hatten Vermutlich zutreffend war auch, dass wohl eine große Mehrheit dieser neuen Eliten durchaus mit den politischen Grundvorstellungen der Erben Mosaddegs sympathisierte. Allerdings - und hier zeigte sich die Schwäche der Argumentation Millers - blieb die große Masse dieser technokratischen Eliten politisch passiv und vermied jedes Engagement. Die Proponenten der New Men, laut Miller die ,emerging future leaders of Iran“, waren denn auch niemand andere als die bereits bekannten Mitglieder des ehemaligen pro-amerikanischen Netzwerks und einige jüngere, aber immer moderate Vertreter der ehemaligen Nationalen Front(en) wie z.B. die MahdawiBrüder. ${ }^{309}$ Das Problem der Umsetzung eines solchen Programms für die amerikanische Politik umging Miller zudem fast vollständig. Notwendig sei für die kommenden drei Jahre, so Miller lapidar, ,[...] to point out gradually to the Shah that in the American view a planned transition to a constitutional monarchy is in his best interests, and the best interest of his country. “310

Angesichts der Entwicklung der amerikanischen Iranpolitik seit 1962 kann nicht überraschen, dass Millers Ideen auf wenig Gegenliebe stießen. Botschafter Holmes, grundsätzlich ablehnend gegenüber jeder Form des reformistischen Interventionismus, reagierte negativ auf die Vorschläge Millers. Zwar sehe er ebenfalls das Potential dieser Gruppe, doch sei aus Sicht amerikanischer Interessen eine Machtübergabe an diese Gruppe kaum geboten. Zudem verwahrte Holmes sich, sicher zurecht, gegen den Mythos amerikanischer Omnipotenz in Iran: „Nor do I agree with their appraisal, which you seem to accept, of the degree of U.S. influence and authority in the domestic affairs of Iran." ${ }^{311}$ John Bowling, einer der einflussreichsten Irankenner der vergangenen Jahre im State Department, der GTI Mitte 1964 verlassen hatte, war noch negativer als Holmes gegenüber Millers Thesen eingestellt. Er beschuldigte Miller,

\footnotetext{
308 Vgl. Miller Study, S. 14; für die „,neuen Männer“ war die amerikanische Unterstützung entscheidend für den Fortbestand des Schahregimes: „They believe he would fall from power if we withdrew our support. As a consequence, they regard the regime as a satellite to American strategic designs."; ebd., S. 16.

${ }^{309}$ Miller Study, S. 45.

310 Ebd., S. 46; ähnlich argumentierte Polk in seinen bereits genannten Memoranden über die Folgen der Transformationen in der Region. Seine Politikempfehlung bezüglich der ,vor-revolutionären“ Staaten lautete: „We cannot stop the modernization process, nor should we try to do so, but modernization without accommodation will lead to revolution. Therefore, we must take a more active political role if we are to be successful."; Polk Memo, 1.2.65, ohne Folder, Lot 69 D 225, NEA/IRN: 1964-66, Box 6 \{Hervorheb. i. Orig. $\}$.

${ }^{311}$ Holmes plädierte gegen die Unterstützung bestimmter politischer Fraktionen innerhalb der Opposition und für die Beibehaltung der bisherigen ,,vorsichtigen“ Politik der Beeinflussung des Reformkurses durch Beratung und Unterstützung. Siehe Holmes Letter, 1.9.64, ,From the Ambassador`, Lot 69 D 30, NEA/IRN: 1964-66, Box 1.
} 
historischen Mythen über die jüngere Vergangenheit Glauben zu schenken, was sich wiederum negativ auf seine Analyse und seine Politikempfehlungen auswirke: „Beyond this, I noted an underlying assumption that somewhere in Iran there are a bunch of loveable liberal democrats who provide a viable alternative to the Shah. ${ }^{\text {“312 }}$ Bowling spitzte die Mehrheitsmeinung im State Department drastisch zu: "The maintenance of the Shah in power should be the first, primary, and only major objective of the U.S. in Iran, and perhaps in the whole Middle East. “313

Dieser Geist beherrschte auch die formale Neuformulierung der Iranpolitik Mitte der Dekade. Die letzte allgemein gehaltene Definition der US-Strategie gegenüber Iran war die bereits ausführlich behandelte offizielle Antwort auf NSAM No228 vom April 1963 gewesen. ${ }^{314} \mathrm{Im}$ August 1964 hatte bereits eine Stimme aus GTI das Fehlen eines allgemeinen ,policy framework" bezüglich Irans moniert. Die Entscheidungen der letzten Monate in der Iranpolitik hätten sich aus „bureaucratic wrangling and maneuvering“ ergeben, ohne Rückkopplung an eine grundlegende Abwägung der eigenen Interessen und Zielsetzungen. ${ }^{315}$ In der JohnsonAdministration hatte sich die bisherige Konzentration der Außenpolitikformulierung im Weißen Haus im Kern erhalten. Gleichzeitig aber akzeptierte man, wenn auch widerwillig, die Rufe nach einer zumindest formalen Niederlegung der zentralen außenpolitischen Zielsetzungen in National Policy Papers (NPP) für als strategisch bedeutsam erachtete Staaten. NSAM N²81 vom Februar 1964 etablierte diese neuen Studien als Abkehr vom geerbten AdHocismus der Kennedy-Administration, obgleich der Enthusiasmus des Weißen Hauses für diesen neuerlichen Anlauf der Strategieformulierung spürbar begrenzt war. ${ }^{316}$ Hintergrund der neuen Initiative waren Bemühungen Rostows vom Policy Planning Council aus, die von McNamara im Pentagon eingeführten Budget- und Planungsreformen, bekannt als Planning, Programming, and Budgeting System, nun auch auf andere Behörden der US-Administration

\footnotetext{
312 Bowlings Kritik bezog sich allerdings auf eine Analyse des monarchischen Systems durch Miller vom Mai 1964, die weiterhin klassifiziert ist. Bowling Letter, 26.5.64, ,EDX - Edu[...]64/1 ', Lot 69 D 30, NEA/IRN: 196466 , Box 2; insgesamt wirke der Text auf ihn als habe ihn ein , [...] very brilliant, very conscientious Iranian intellectual with a NF $\{$ National Front; R.P. $\}$ background who had promised his professor to write a completely unbiased essay on the Shah" verfasst. Ebd. \{Hervorheb. i. Orig.\}; Martin Herz reagierte ungehalten auf Bowlings Kritik, die er als ad hominem empfand: „I wonder if your comments about our report on the Shah would have been the same if the drafting credit had gone, not to Bill Miller, but to an FSO \{Foreign Service Officer; R.P.\} Gerhard Schnickelfritz."; Herz beschwerte sich dagegen in seinem Gegenangriff über die offenkundig unterlegten „underlying assumptions“ seitens GTI, wonach jedes Nachdenken über Alternativen zum Schah nicht mehr akzeptabel sei: ,What kind of 'underlying assumptions' does GTI wish to see reflected in our analytical reporting about the Shah? If he is irreplaceable, does that mean he has no weaknesses?"; Herz Letter, 3.6.64, ebd.

${ }^{313}$ Sollten die Nationalisten in Iran an die Macht kommen, hätte das neue Regime ,, as its primary function the discomfiture of the United States.“; Bowling Letter, 26.5.65, ,POL 1 Gen. [... ]65', Lot 69 D 490, NEA/IRN: 196466, Box 12 \{Hervorheb. v. Verf.\}.

${ }^{314}$ Formal waren offenbar auch weiterhin die Guidelines vom September 1962 in Kraft.

${ }^{315}$ Vgl. Thomas Memo, 17.8.64, ,POL 1-2 Bas[...]64‘, Lot 69 D 225, NEA/IRN: 1964-66, Box 6; als das zentrale Problem, für das es allgemeiner Orientierung bedürfe, nannte Thomas die Frage der inneren Verfasstheit Irans nach dem Abgang des Schahs und eine mögliche Vorbereitung auf dieses Szenario zum gegenwärtigen Zeitpunkt, wie z.B. den Aufbau einer Machtalternative zum gegenwärtigen autoritären System.

316 Die National Policy Papers waren definiert als ,,comprehensive, authoritative and unifying statements of US policy"; Komer warnte sogleich intern, es könne der falsche Eindruck einer umfassenden Rückdelegation der Außenpolitik an das State Department entstehen. Offenbar wurde NSAM N ${ }^{\circ} 281$ als Zugeständnis an Rostows fortgesetztes Drängen nach Grand Strategy-Formulierungen nach dem Muster der BNSP angesehen. Vgl. ed. note, FRUS 64-68, XXXIII, S. 9; Komer warnte vor einer neuerlichen ,paper mill“ wie in der EisenhowerAdministration. Komer Memo, 1.4.64, ebd., S. 14f.; vgl. auch Memo for the Record, 20.4.64, ebd., S. $15 f . ;$ vgl. PrADOS, Keepers of the Keys, S. $161 \mathrm{f}$.
} 
auszudehnen, eben auch auf das State Department und die Außenpolitikformulierung. Statt nur vage Orientierung hinsichtlich amerikanischer Interessen $\mathrm{zu}$ bieten, sollte das neue Comprehensive Country Programming System, von dem die NPPs nur ein Teil waren, direkte Handlungs- und Umsetzungsanweisungen für amerikanische Offizielle und Diplomaten liefern. $^{317}$

Ab dem Sommer 1965 wurde auch die Abfassung eines NPP für Iran in Angriff genommen. Die Ausformulierung des NPP ging indes nur schleppend voran und zog sich bis zur Verabschiedung des Papiers im Februar 1967 über mehr als anderthalb Jahre hin, wobei die langen Verzögerungen weniger ein Ausdruck interbürokratischer Auseinandersetzungen waren. $^{318}$ Tatsächlich war das Interesse des Weißen Hauses, aber auch anderer Behörden, am NPP-Prozess eher begrenzt und die von Rostow und anderen Initiatoren erhoffte integrierte Politikformulierung, -programmierung und -implementierung als Folge der neuen Prozesse stellte sich zu keinem Zeitpunkt ein. Im Resultat ähnelte das Comprehensive Country Programming System ähnlichen, letztlich nur peripher bedeutsamen Unternehmungen in der Vergangenheit, wie z.B. den Guidelines aus der Kennedy-Ära. ${ }^{319}$ Harold Saunders sprach später von dem ,you don't find policy on paper“-Konzept der Johnson-Administration. Gemeint war eine sich fortsetzende Verhandlung angestrebter Politikziele und deren Implementierung in einem dauernden, aber auch flexiblen Austausch zwischen Ideen und Personen, anstelle einer starren Ausformulierung strategischer Zielsetzungen. ${ }^{320}$

Das Produkt der Beratungen ist dennoch für die vorliegende Arbeit von Interesse, spiegelt es doch die Entwicklung des strategischen Denkens hinsichtlich Irans Mitte der 1960er Jahre wider. Die verabschiedete Version des NPP für Iran ist ein klassisches Kompromissprodukt nach monatelangen Abstimmungsprozessen zwischen verschiedenen Behörden mit unterschiedlichen Interessenlagen. Verschiedene Sichtweisen wurden integriert, sogar in Teilen die kritische Sichtweise eines Bill Millers ${ }^{321}$ - wobei die Ansichten bei weitem nicht

\footnotetext{
${ }^{317}$ Vgl. Crockett Memo, 12.2.65, FRUS 64-68, XXXIII, S. 48-53; Planning, Programming, and Budgeting System wird häufig als ein kybernetisch inspirierter, formaler Versuch interpretiert, strategische Planung mit Haushaltsplanung direkt zu verbinden. Vgl. LeACACOS, Fires in the In-Basket, S. 17, 360f.

${ }_{318}$ Der erste Teil des Endresultats, verabschiedet von Rusk am 2. Februar 1967, ist in Teilen abgedruckt in FRUS 64-68, XXII, S. 341-51; der zweite Teil des NPP war eine „Staff Study“ ohne formale Zustimmung der beteiligten Behörden. Das Gesamtdokument mit beiden Teilen und mit allen Annexen und über 100 Seiten findet sich in den Beständen des Policy Planning Council. Siehe National Policy Paper: Iran, 2.2.67, ,Near and Middle East 196566', Lot 72 D 139, S/P Records, Subject and Country: 1965-69, Box 315 im Folgenden zitiert: NPP Iran, Part I/Part II $\}$; frühere Entwürfe finden sich in den GTI-Akten. Vgl. First ,Regular' Draft National Policy Paper on Iran, 26.11.65, ,POL 1-2 Bas[...]65', Lot 69 D 490, NEA/IRN: 1964-66, Box 12; Draft NPP Iran, 14.3.66, ,POL 1-2 Bas[...]66“, Lot 70 D 330, ebd., Box 17 \{im Folgenden: NPP Draft \#1 und \#2\}.

${ }^{319}$ Insbesondere das Bureau of the Budget kritisierte das neue System scharf. Vgl. Rostow/Crockett Memo, 7.3.66, FRUS 64-68, XXXIII, S. 141-6; Thomas Schelling, der bekannte Spieltheoretiker und strategische Denker, lehnte das Angebot einer Assistant Secretary-Stelle im State Department, betraut mit der Koordination dieser Bemühungen, ab. Vgl. Rostow Memo, 10.3.67; CIA Memo for the Record, ebd., S. 259-63, $281 \mathrm{f}$.

${ }^{320}$ Saunders Paper, 15.3.68, ebd., S. 405.

321 Tatsächlich basierte der Part II des NPP auf einer zuvor von Miller und zwei weiteren Autoren für INR abgefassten Studie zur politischen Lage in Iran vom Dezember 1966. Vgl. INR Study ,Studies in Political Dynamics Number 13: Iran', Dezember 1966, DNSA: IR00603; siehe auch William Green Miller OHI, 10.2.2003, FAOH; die Interpretation Bills, wonach Millers Analyse „little-known and greatly ignored“ gewesen sei, ist somit kaum haltbar. Vgl. BILL, Eagle and Lion, S. 169.
} 
mehr so unterschiedlich waren wie in der Vergangenheit - mit der Folge eines sehr inkohärenten Endprodukts mit begrenzter Aussagekraft über die tatsächlich verfolgte Politik der Johnson-Administration. ${ }^{322}$ Das NPP für Iran definierte die amerikanischen Interessen in Iran sowohl hinsichtlich der eigenen breiteren strategischen Interessen - fortgesetzter Zugang $\mathrm{zu}$ iranischen Kommunikations- und Transporteinrichtungen, Überflugrechte, Abhöreinrichtungen und Ölressourcen - und dem langfristigen Interesse an der Stabilität eines Irans mit pro-westlicher Ausrichtung. Hinsichtlich des Letzteren wurde ein amerikanisches Interesse an iranischer Eigenständigkeit in außenpolitischen Belangen expliziert, die Akzeptanz der Unabhängigkeitsbestrebungen in der jüngeren Vergangenheit reflektierend. Zudem wünschte man sich eine vernünftig und effizient gemanagte iranische Volkswirtschaft mit ausgewogener Ausgabenverteilung zwischen ökonomischer Entwicklung und militärischer Modernisierung, den Grundgedanken hinter der Annual Review wiedergebend. Die ehemals ambitionierten Ideen einer politischen Transformation im Sinne des reformistischen Interventionismus der frühen 1960er Jahre aber waren nunmehr einem - allerdings reichlich unkonkreten - Wunsch nach Aufbau „stärkerer Institutionen“ zur politischen Partizipation gewichen. ${ }^{323}$ Diese moderat-programmatische Passage wurde aber sogleich ausbalanciert durch die Feststellung, man favorisiere zwar keine spezifische Form innerer Verfasstheit für Iran, aber dass , $[\ldots]$ the monarchy as conducted by the present Shah affords the best available means for safeguarding our basic interests." ${ }^{\text {(324 }}$ In der sprachlich etwas abgeschwächten Endversion des NPP wurden Zuspitzungen früherer Versionen entfernt, wonach der Schah gegenwärtig sogar der „linchpin for the safeguarding of our basic security interests“ sei - im Übrigen eine fast identische Übernahme einer Formulierung aus der „U.S. Strategy for Iran“ vom Frühling 1963, was wiederum die prinzipielle Kontinuität der US-Politik im Nachgang der Aufgabe des reformistischen Interventionismus exemplifiziert. ${ }^{325}$ Dennoch besteht trotz aller Abwägungen kein Zweifel an der weitgehenden Identifikation amerikanischer Politikziele mit dem Fortdauern der persönlichen Herrschaft Mohammad Rezās.

Im Dokument finden sich Spuren der traditionellen reformistischen Rhetorik und der grundsätzlichen Sorge über die langfristige Entwicklung. Das NPP für Iran betonte zwar die

\footnotetext{
${ }^{322}$ So verzichtete das Country Team vor Ort auf allzu einschneidenden Kritiken, um den Prozess nicht aufzuhalten, was wiederum die dem NPP zugeschriebene Bedeutung widerspiegelt. Vgl. Herz Letter, 28.4.66, ,POL 1 Gen[...]66“, Lot 70 D 330, NEA/IRN: 1964-66, Box 18.

323, An independent and increasingly self-reliant Iran, free from any foreign domination or aggression, and motivated to cooperate with the West", war nun als amerikanisches Politikziel definiert. NPP Iran, Part I, S. 3 [FRUS 64-68, XXII, S. 342].

${ }^{324}$ NPP Iran, Part I, S. iv.; an anderer Stelle sprach das NPP davon, dass US-Interessen ,are pursued within the framework of our particular relationship with the monarchy of that country."; ebd. [FRUS 64-68, XXII, S. 342] ein früherer Entwurf hatte noch von einer ,peculiar relationship“" gesprochen. Vgl. NPP Draft \#1, S. 2 \{Hervorheb. i. Orig. $\}$.

${ }^{325}$ NPP Draft \#1, S. 2; selbst in diesem Entwurf findet sich die Abschwächung, dass - trotz aller Vorteile - die „,maintenance of the Shah's regime of the monarchy as an institution is not necessarily an intrinsic long-term United States objective“; ebd.; die „Linchpin“-Passage wurde in der Endversion abgeschwächt zu: „While the United States is not necessarily committed to the support of any particular form of Government in Iran, the Shah at present affords the best means for the safeguarding of our basic security interests in Iran and is the only personality on the scene who can lead the anarchically-bent Persians."; NPP Iran, Part I, S. 2 [FRUS 64-68, XXII, S. 342]; zur US-Strategie von 1963 vgl. oben S. 378ff.
} 
gegenwärtige Stabilität Irans - gerade im Vergleich zu den vorhergehenden Jahren -, wies aber dennoch auf die absehbaren destabilisierenden Effekte der Divergenz zwischen sozioökonomischer und politischer Entwicklung in der Zukunft hin, im Kern die alte These William Polks. So explizierte das NPP als ,hauptsächliches politisches Problem“ und auch als Grundlage für das längerfristige politische Überleben des Regimes die Integration der gegenwärtig apathischen, andersdenkenden oder offen feindseligen Teile der Bevölkerung: „Otherwise, the regime will be obliged to carry on with its present narrow and only slowly growing base of support, sustained by the strength of the armed forces, in which case its prospects for stability and growth over the long run would not seem to be good."326 Das NPP warnte zugleich vor einseitigen politischen Strategieentwürfen wie einer Politik des ,all-out support of the Shah, without reservation“ - im Grunde die Position John Bowlings und die eigentliche amerikanische Politik in der Zukunft - oder einer Politik der ,withdrawal of support“ vom Regime. ${ }^{327}$ Versuche, fortgeschrittene westliche Demokratie-Konzepte auf Iran $\mathrm{zu}$ übertragen, seien angesichts der fehlenden Fundamente und bisherigen politischpartizipatorischen Erfahrungen zum Scheitern verurteilt. Dennoch müsse für die Zeit nach dem gegenwärtigen Schah und für andere Eventualitäten vorgesorgt werden. Daher wurde der Aufbau sozialer, ökonomischer und politischer Institutionen als ,means for orderly and peaceful transfer of power, as necessary, and in the longer term facilitate increased participation of ever-widening sectors of society in their own government" explizit als Politikziel aufgenommen. ${ }^{328}$

Im Kern also blieb auch im Jahr 1967 die Kernaussage der früheren reformistisch inspirierten Sichtweise des iranischen Entwicklungswegs, wenn auch weitaus weniger alarmistisch, erhalten. Allerdings folgte aus Sicht der Johnson-Administration daraus - und im Gegensatz zur Perspektive eines Polks oder Millers -keine zwangsläufige Vorgabe für ein Eingreifen der USA. Vielmehr stellten all dies Herausforderungen dar, die überwiegend oder sogar vollständig von den Iranern selbst zu meistern waren. Vorstellbar für die Autoren des NPP war maximal eine amerikanische „Aufforderung“ an den Schah, im Kontext der anstehenden Mağles-Wahlen ein „limited opening up“ des politischen Systems - die Zulassung einiger ausgewählter

\footnotetext{
${ }^{326}$ NPP Iran, Part I, S. 9.

${ }^{327}$ Ebd., S. 15; die erstere Alternative ,would leave us totally without flexibility in a period of dynamic change“, die Konsequenzen im Falle eines plötzlichen Umbruchs meinend, die letztere Alternative dagegen ,,would deprive us of important political and strategic assets without any offsetting gains in Iran or elsewhere. “; der erste Entwurf enthielt noch einige Seiten Diskussion über die Vorzüge der verschiedenen Politikalternativen. Bezeichnend ist auch die Ablehnung eines Mittelwegs zwischen den beiden skizzierten Extrempositionen. Es gebe kaum,,evidence to suggest that the nationalists would be satisfied with such a gradualist approach, nor, indeed, that they could do better than the Shah in developing political institutions vital to long-term growth."“; NPP Draft \#1, S. 17; dies ist im Kern die (Bekräftigung der) Ablehnung der alten Task Force-Politik von 1961.

${ }^{328}$ NPP Iran, Part I, S. 4 [FRUS 64-68, XXII, S. 343]; die bisherigen Versuche, politische Parteien zu etablieren, seien allesamt gescheitert. Ein konkreter Vorschlag aus dem Policy Planning Council vom Januar 1966 empfahl den graduellen Aufbau eines tentativen Zweiparteiensystems mithilfe politischer Kontrolle und möglichen Wahlrechtsbeschränkungen, unter Einschluss einer reformierten und nunmehr regime-loyalen Nationalen Front. Vgl. Policy Planning Council Study, 24.1.66, ,Near and Middle East, 1965-66،, Lot 72 D 139, S/P Records, Subject and Country: 1965-69, Box 315; Howison Memo, 24.2.66, ,POL 1 Gen[...]66', Lot 70 D 330, NEA/IRN: 196466, Box 18; die „Divergenz"-These blieb ebenso fester wie folgenloser Bestandteil der Berichterstattung der Botschaft. Vgl. Tehran \#A-123, 8.7.75, Asnād 7, S. 154-73.
} 
Oppositioneller nach altbewährtem Muster - in Erwägung zu ziehen. ${ }^{329}$ Die amerikanische Zurückhaltung war weniger einer Einsicht in die grundsätzlichen Schwierigkeiten jedweder Intervention in die inneren Angelegenheiten anderer Staaten oder Gesellschaften oder gar moralischen Bedenken geschuldet - tatsächlich intervenierte die Johnson-Administration zur gleichen Zeit offen wie verdeckt in einer ganzen Reihe von Staaten. Ausschlaggebend für die Nichteinmischung in Fragen der iranischen Innenpolitik war vielmehr die kaum zu bestreitende Tatsache des amerikanischen Einflussverlusts in Iran. Abgesehen vom militärischen Bereich, so das NPP, werde man in Zukunft weitaus weniger in den inneren iranischen Macht-Zirkeln präsent sein und weniger eingebunden in die eigentlichen Entscheidungsprozesse als in der Vergangenheit: ,[...] we will move more into the role of a trusted ally (hopefully still the most trusted) and away from the earlier role of responsible senior partner. ${ }^{630}$ Man hatte somit die Begrenzungen des eigenen Einflusses in Iran akzeptiert. ${ }^{331}$ Angesichts der fast uneingeschränkten Machtposition Mohammad Rezās und der allgemeinen Aufbruchsstimmung waren amerikanische Einflussmöglichkeiten nun in der Tat äußerst limitiert: „Unless the booming economy takes a turn for the worse or the political dissidents display unaccustomed effectiveness, the Shah's confidence in his own ability to rule and manage his nation is not likely to be shaken by advice and admonition from even the friendliest of critics. “332

Wie gedachte man aber nun die als Politikziel für die kommende Dekade ausgegebene Entwicklung hin zu einer, ,new but still mutually rewarding relationship between the United States and Iran" zu bewerkstelligen? ${ }^{333}$ In erster Line war - wenig überraschend - eine Fortsetzung des bisherigen Kurses vorgesehen, basierend auf der engen Kooperation mit der iranischen Führung und positiven Akzeptanz der neuen Unabhängigkeit. Die neue Gleichrangigkeit betonend, plädierte das NPP für das Festhalten an der Einstellung der Entwicklungshilfe angesichts der iranischen wirtschaftlichen Fortschritte. Ein Schwerpunkt für die erneuerten Beziehungen wurde trotz der Beseitigung des US-Monopols im militärischen Feld verortet, nicht zuletzt aufgrund der weiterhin gegebenen Dominanz amerikanischer Rüstungsgüter und Trainingsunterstützung. ${ }^{334}$ Als Ziel wurde die Fortsetzung der verschiedenen Militärmissionen und der Positionierung der USA als ,primary foreign military influence " in Iran ausgegeben. ${ }^{335}$ Des Weiteren strebte man in ökonomischer Hinsicht nach einem ,appropriate share of the growing commercial market“ für US-Unternehmen und nach

\footnotetext{
${ }^{329}$ NPP Iran, Part I, S. 20 [FRUS 64-68, XXII, S. 346].

${ }^{330}$ NPP Iran, Part I, S. 19 [FRUS 64-68, XXII, S. 345] \{Hervorheb. v. Verf.\}; etwas optimistischer klang es im zweiten Teil des NPP: „US influence on Iranian domestic and foreign policy will not melt away solely because the economic aid program is being phased out. US support for the Shah is still important to his regime. [...] Thus the US will probably retain much of its entrée into Iranian affairs despite the xenophobic secondary effects of Iran's increasingly independent stance."; NPP Iran, Part II, S. 53.

331 Eines der wenigen Dokumente, das die internen Debatten über das NPP widerspiegelt, drehte sich auch maßgeblich um die Frage des tatsächlich verbliebenen Einflusses. Die Mehrheit ging dabei von geringem „US leverage" in Iran aus. Es gab aber auch durchaus Kritik an der zu starken Konzentration auf den Schah im NPPDraft. Vgl. Crawford Memo, 2.12.65, ,POL 1-2 Bas[...]65‘, Lot 69 D 490, NEA/IRN: 1964-66, Box 12.

${ }_{332}$ NPP Iran, Part I, S. iv.

${ }^{333}$ Vgl. NPP Iran, Part I, S. 3 [FRUS 64-68, XXII, S. 342] \{Hervorheb. v. Verf.\}.

${ }^{334}$ Vgl. ebd., S. 17 [FRUS 64-68, XXII, S. 344].

${ }^{335}$ Vgl. ebd., S. 25 [FRUS 64-68, XXII, S. 349] \{Hervorheb. v. Verf.\}.
} 
Einfluss in Schlüsselbereichen der iranischen Wirtschaft. ${ }^{336}$ Zudem kündigte man trotz Einstellung der sonstigen Wirtschaftshilfe die Fortsetzung der Ausbildungshilfe für Aufstandsbekämpfung für die iranische Armee und die anderen Sicherheitsorgane an. Gerade Letzteres stand emblematisch für die Identifikation der amerikanischen Iranpolitik mit der repressiven Herrschaft der Pahlawis. Die aktive Mitwirkung an der weiteren Professionalisierung und Steigerung der Effizienz der Unterdrückungsorgane strafte alle Lippenbekenntnisse im NPP zum wünschbaren Aufbau politischer „Institutionen“ oder einem vorsichtigen ,opening up“ Lügen. ${ }^{337}$

Die Konsequenzen der Fortsetzung der bisherigen Politik der engen Identifikation mit der autoritären Herrschaft Mohammad Rezās wurden allerdings nicht weiter erläutert. Das Selbstbild der eigenen, vorwiegend altruistischen Motive vernebelte das zu dieser Zeit durchaus von aufmerksamen Beobachtern registrierte Aufkommen anti-amerikanischer Ressentiments in breiten Bevölkerungsschichten. So erklärte das NPP, die enge Assoziierung der vergangenen Jahre habe zwar gewisse Makel am Bild der USA entstehen lassen, aber ,[...] we have not felt the full blast of blame of the foreign devils which Iranians have on occasion aimed at other nationalities.“338 Bill Miller hatte in einer parallelen INR-Studie die Hoffnung geäußert, die Unabhängigkeitsbestrebungen Mohammad Rezās würden zumindest das amerikanische Image des hinter den Kulissen agierenden Strippenziehers revidieren. ${ }^{339}$ Zugleich betonte man im NPP aber die Pfadabhängigkeit der bisherigen Entwicklung. Für das kommende Jahrzehnt lasse sich eine Fortsetzung der engen Beziehungen mit dem nun weitgehend unabhängig agierenden Iran vorhersehen, trotz der periodisch wiederkehrenden atmosphärischen Störungen, die jedoch nie wirklich mit politisch substantiellen Fragen befasst seien. ${ }^{340}$ Ein Wandel dieser besonderen Beziehungen $\mathrm{zu}$ Iran sei eigentlich nur durch ein Wiederaufleben einer breiten antimonarchisch-nationalistischen Oppositionsbewegung $\mathrm{zu}$ erwarten. Allerdings würde das bislang Erreichte auch in einem solchen Szenario vorteilhaft wirken, zumal die „geopolitischen“ Gegebenheiten im Grundsatz das iranische Verhalten determinierten:

While the course of such a movement is essentially unpredictable, we can take some heart from the fact that the imperatives of Iranian foreign and domestic policy could be counted upon to impel any but the most blatantly pro-Soviet regime to maintain an important balancing relationship with a Free World power. ${ }^{341}$

Interessant an diesem Zitat ist, dass in einer vorherigen, in GTI formulierten Version des NPP noch anstelle eines „eklatant pro-sowjetischen Regimes“ von einem „most intransigently

\footnotetext{
${ }^{336}$ NPP Iran, Part I, S. 30.

${ }^{337}$ Der zweite Teil des NPP, maßgeblich von Miller geprägt, enthielt dagegen Passagen, die sich für eine größere Distanz zum Regime aussprachen, für eine Position der „,mutual independence“ im gegenseitigen Verhältnis und für die Beibehaltung von Kontakten zur nationalistischen Opposition auch gegen das Missfallen der Führung. Vgl. NPP Iran, Part II, S. 54.

${ }^{338}$ NPP Iran, Part I, S. 5.

${ }^{339}$ Vgl. INR Study ,Studies in Political Dynamics Number 13: Iran', Dezember 1966, DNSA: IR00603, S. iv.

${ }^{340}$ Vgl. ebd., S. 5.

${ }^{341}$ Ebd., S. 6 f.
} 
xenophobic Iranian regime“ die Rede gewesen war. ${ }^{342}$ Offenbar glaubten die amerikanischen Verantwortlichen, selbst im Falle eines gewaltsamen Umsturzes könnten die eigenen Interessen gewahrt werden - die im NPP erwähnte Möglichkeit einer Machtübernahme einer prosowjetischen Fraktion war indes so unwahrscheinlich, dass man sie fast ausschließen konnte. Im Gegensatz zur vorherigen Vormacht in Iran, Großbritannien, fiel es den Offiziellen in Washington bemerkenswert schwer, sich die eigene Unpopularität einzugestehen bzw. die teilweise sogar offen zutage tretende Feindseligkeit gegenüber dem bisherigen Patron angemessen $\mathrm{zu}$ registrieren, obgleich zahlreiche Berichte der Botschaft dieses Phänomen beschrieben.

Dabei war der wachsende Anti-Amerikanismus in Iran kaum noch zu übersehen. Trotz des Niedergangs amerikanischen Einflusses und des Zurückfahrens des Engagements war augenfällig, wie merklich die Popularität der USA in Iran Mitte der 1960er gesunken war. Die US-Botschaft berichtete besorgt, prominente Iraner suchten jede Nähe zu den USA zu meiden, aus Angst vor gesellschaftlicher Ächtung. So habe sich beispielsweise die Association of Graduates of American Universities in Association of University Graduates umbenannt. ${ }^{343}$ Aus den Provinzen berichteten die Konsulate, aus Sicht der lokalen Bevölkerung sei der „honeymoon with America [...] now over“. Es herrsche die Meinung vor, die vielen amerikanischen Hilfsprogramme in Iran in den vorausgegangenen Jahren hätten nur sehr begrenzten Erfolg gezeitigt. ${ }^{344}$ Auffallend war der nun rasch einsetzende Prestigeverlust der USA unter den iranischen Oppositionellen. Lange als größte Hoffnung für eine demokratische Transformation angesehen, wandelte sich das Bild der USA in den Augen gebildeter Iraner mehr und mehr zu dem einer in erster Linie auf die eigenen strategischen Interessen bedachten und den freiheitlichen und nationalistischen Ambitionen Irans im Kern feindselig gesinnten, fremden Macht. Die USA wuchsen Mitte der 1960er Jahre im Kontext iranischer politischer Mythologie in die Rolle hinein, die traditionell Großbritannien vorbehalten gewesen war. Ironischerweise zu dem historischen Zeitpunkt, als sich Mohammad Rezā vom amerikanischen Patron erfolgreich emanzipierte, entstand nun in einer breiten Öffentlichkeit die Wahrnehmung der USA als eigentlicher Strippenzieher hinter dem Regime und als Urheber der immer stärker werdenden politischen Repression im Land. Bereits 1964 konstatierte die Botschaft, dass sich in Iran ein „Myth of American Omnipotence“ etabliert habe, der mit dem realen Einfluss der

\footnotetext{
${ }^{342}$ Vgl. NPP Draft \#1, S. 6f.; der zweite Teil des NPP enthält Passagen zur Rolle der Religion in Iran: „,the general effect of religion in internal politics has been inconclusive. Purely religious fanaticism is rare in Iran. "; NPP Iran, Part II, S. 2; eine ähnliche Auffassung vertrat Edward H. Thomas, zu dieser Zeit amerikanischer Konsul in Maschhad.: "If somehow a popular government were to come to power some day, our position might be considerably more uncomfortable than now, at least for a time, because of the likelihood such a government would have a demagogic, xenophobic nature. Even then, however, it would be unduly pessimistic to assume that we could not come to terms with the changed situation."; Meshed \#47, 23.6.63, 788.00/6-2362, CF 60-63; eine ähnliche Einschätzung findet sich auch in Dokumenten aus den Folgejahren immer wieder, z.B.: „American support against Iran's perceived principal enemy makes its relationship equally vital, if not more so, to Iran's present government and probably to any future government except an extreme radical one such as Libya's present regime (very unlikely, but not impossible)."; Tehran \#A-123, 8.7.75, Asnād 7, S. 154-73, hier S. 173.

${ }^{343}$ Tehran \#A-164, 22.8.66, POL 2-3 IRAN, CF 64-66.

${ }^{344}$ Vgl. den Bericht der allgemeinen Stimmung in Isfahan in Tehran \#A-223, 29.9.65, POL 2 IRAN, CF 64-66.
} 
Supermacht in Iran so gut wie gar nichts gemein habe: „By being given credit today for power to influence the situation in Iran that we do not actually possess, however, we of course also incur the blame for deficiencies that we are in no position to prevent or remedy. “ ${ }^{\star 345}$

Die nach der Revolution verfügbaren Quellen widersprechen dem Mythos eines von der CIA de facto kontrollierten Staatsapparates. Mohammad Rezā hatte in den Jahren seit 1961 nicht nur das zuvor politikrelevante pro-amerikanische Netzwerk unterminiert und praktisch ausgeschaltet, sondern auch die bisherigen Freiräume der US-Berater und der CIA erheblich eingeschränkt. Angesichts der Quellenlage nicht zu klären ist das verbliebene Ausmaß der geheimdienstlichen Penetration der Verwaltung und des politisch-strategischen Establishments in Iran durch die CIA. Ein Bericht des Botschafters an die Special Group (CI) erwähnte die Verfügbarkeit von Quellen innerhalb der iranischen Regierung in ,ausreichendem“ Ausmaß. ${ }^{346}$ Angesichts der historischen Genese der amerikanischen Position in Iran und der Rolle, die die USA gerade in hochsensiblen Bereichen lange Zeit spielten, gerade was den Aufbau indigener geheimdienstlicher Strukturen in Iran betraf, ist wohl von einer weitreichenden Penetration auszugehen. Wenngleich dies als Bestätigung iranischer politischer Folklore erscheinen mag, so ist dennoch ein Caveat angebracht. Zwar kann davon ausgegangen werden, dass die USA über hervorragende Informationsquellen innerhalb der iranischen Staatsorgane verfügten, die es ermöglichten zu einem sehr frühen Zeitpunkt über interne Pläne und Politikwechsel in Kenntnis gesetzt zu werden. Dieser Informationsvorsprung darf allerdings nicht mit politischem bzw. strategischem Einfluss gleichgesetzt werden. Die Geschichte der

\footnotetext{
345 Tehran \#A-702, 15.6.64, POL 1 IRAN, ebd.; der Botschaft zufolge gäbe es weit verbreitete Vorstellungen, die USA entschieden über die Besetzung der wichtigsten Regierungsposten. Parlamentarier suchten US-Offizielle um Rat, ob z.B. eine kritische Rede zu einem bestimmten Aspekt iranischer Regierungsführung angemessen bzw. zu empfehlen sei. Ähnliche Überzeugungen brachte auch eine informelle Befragung Teheraner Taxifahrer zum Ausdruck. Vgl. Tehran \#A-323, 21.12.64, POL 2 IRAN, CF 64-66; nach der Revolution wurde man sich des Ausmaßes der anti-amerikanischen Gefühle bewusst. Viele Iraner würden den US-Einfluss als ,,all-pervasive and all-powerful“" beschreiben: „Anti-Americanism is rooted in 1) our support of the Shah and, by extension, the excesses of his government, 2) our identification with the now negatively perceived sense of westernization and materialism; 3) our image as arms purveyor and super salesman of the 'expensive and the unnecessary' combined with a 'colonialist'/'imperialist' image stemming from the former presence of thousands of Americans in Iran."; Curran Memo to Director, 9.2.79 Asnād 6, S. 559; ein früherer iranischer Offizieller stellte den AntiAmerikanismus auch nach der Revolution als Randphänomen dar. Vgl. AMUZEGAR, Dynamics, S. 46-51.

${ }_{346}$, ,Most of them are official GOI \{Government of Iran; R.P.\} sources which under all conditions might not be entirely reliable. [...] embasy $\{$ sic $\}$, consulates, \{one word not declassified; wohl CIA bzw. CAS \} have certain capabilities in this field, including the determination of possible motivations behind GOI disclosures."; Tehran \#650, 27.10.65, ,Iran Cables, Vol I., 1/64-12/65‘, NSF, Country File, Box 136, LBJL; diese Passagen sind teilweise geschwärzt in der FRUS-Version. Siehe FRUS 64-68, XXII, S. 183ff.; es nicht ganz klar, worauf Meyer mit den „capabilities“ anspielt, aber es ergibt sich hier zumindest der Eindruck einer Penetration in einem Maße, die eine Irreführung durch die iranische Führung so gut wie ausgeschlossen macht, will man Meyer Glauben schenken. Innerhalb des State Department hatte Iran den Ruf eines der „CIA countries“" wie z.B. auch Südkorea. Vgl. William Green Miller OHI, 10.2.2003, FAOH; später bemängelte man intern in der CIA auch offenbar die Vernachlässigung der elektronischen Aufklärung der iranischen Regierungszentrale und des Schah-Umfelds. Man habe sich auch nicht getraut, eine Quelle in der direkten Umgebung des Monarchen zu rekrutieren. Vgl. WOODWARD, Veil, S. 109f.; den diplomatischen Verkehr Irans lasen die USA mittels manipulierter Chiffriergeräte der Schweizer Firma Crypto AG mit. Vgl. „Der Informant tritt aus dem Schatten“, 17.2.2020, Basler Zeitung, S. 5; im Gegensatz zu den USA verfügten die Sowjets offenbar nur sehr begrenzt über Human Intelligence, also „,menschliche Quellen“ in Iran. Bereits 1944 wurde der Offizier und spätere General Ahmad Mogarribi rekrutiert (1977 vom SAWAK entdeckt und später hingerichtet). Zudem schöpfte man einen wenig ergiebigen Verwandten Howejdās ab. Vgl. ANDREW/MitroKHIN, Mitrokhin Archive II, S. 139ff., 178ff.; 533 n39; ähnlich KuZICHKIN, Inside the $K G B$, S. 104ff., 196-200; so habe man zu keiner Zeit eine Quelle innerhalb des SAWAKS gewonnen. Offenbar aber war Moskau durch Mogarribis zeitweilige Beschäftigung in der Beschaffung gut über die USWaffenverkäufe informiert. Vgl. ebd., S. 196f., 212.
} 
amerikanisch-iranischen Beziehungen im Anschluss an die gescheiterte reformistische Intervention macht offenkundig, dass eben dieser Einfluss der USA rasant abnahm und in den zentralen Fragen für das bilaterale Verhältnis wie z.B. der Umorientierung der iranischen Außenpolitik oder der Ablösung des US-Militärmonopols die klandestine Dimension nur sehr begrenzt von Bedeutung war, zumal die fortschreitende Konzentration politischer Macht in der Person des Schahs den ohnehin begrenzten Nutzen der Quellenanwerbung noch zusätzlich minderte. Die Interpretation Irans in den 1960er Jahren als geheimdienstlich unterwanderte Marionette der USA ist historisch keinesfalls belegbar und widerspiegelt auch nicht im Entferntesten die politische Realität, wenngleich viele Studien dieser Zeit bis heute von einer solchen arkanen Steuerung ausgehen. ${ }^{347}$

Aufmerksame Beobachter warnten vor einem spürbaren anti-amerikanischen Trend unter iranischen Intellektuellen, selbst bei denjenigen, die früher den USA eng verbunden gewesen waren oder sogar dort längere Zeit gelebt hatten. Bekanntestes Beispiel war der Intellektuelle und Poet Ğalāl Āl-e Ahmad, ein ehemaliger Mitstreiter Xalil Malekis und dessen Abspaltung von der Tudeh, der 1962 dem anti-westlichen Reflex mit der Prägung der Begrifflichkeit der Garbzadegi, vielleicht zu übersetzen als „West-Krankheit“ oder „Ver-Westgiftlichung“, einen bald geflügelten Ausdruck gab. ${ }^{348}$ Bill Miller in seiner bereits analysierten Studie zu den New Men in Iran verwandte das Beispiel des Teheraner Universitätsprofessors und Philosophen Sejjed Hosejn Nasr. Nasr hatte am MIT und in Harvard studiert und sich schrittweise von einer akademischen Karriere in den Naturwissenschaften ab- und islamischer Philosophie in seinem Heimatland zugewandt. Zugleich habe Nasr, so Miller, ein zunehmend kritisches Bild der USA und von deren Einfluss in Iran entwickelt: „That someone like Nasr who is so thoroughly educated in Western thought and so sympathetic to Western and particularly American life, should be so critical of the influence of the West in Iran is of great significance. "349 Bezeichnend für die intellektuelle Kritik war die Hinwendung zur iranischen Vergangenheit als Gegenbild zur westlich geprägten Moderne, und insbesondere - in Abgrenzung zur staatlichen

\footnotetext{
${ }^{347}$ Vgl. AZIMI, Quest for Democracy, S. 260-6; ähnlich auch Vali Nasr, der US-Druck für den Niedergang des Einflusses des Wirtschaftsministeriums verantwortlich macht. Vgl. NASR, Late-Pahlavi State, S. 106-8; beispielhaft auch Zonis, der von einer fast vollständigen Steuerung Irans durch die USA auszugehen scheint. Vgl. ZONIS, Majestic Failure, S. 219, 259ff.; selbst Mitte der 1960er Jahre gab es noch vereinzelte Versuche, auf die öffentliche Meinung Einfluss zu nehmen. So gab die Botschaft Geld für das Verfassen von ,unattributed stories under contract" für die Zeitungen mit der größten Auflage und einige kleinere politisch einflussreiche Blätter. Vgl. Embassy Study ,Policy and Program Summary: Iran', 15.11.64, ,Policy and Program Summary Iran', Lot 69 D 225, NEA/IRN: 1964-66, Box 6; offenbar unterhielt der SAWAK eine recht ergiebige Quellen unter dem Decknamen „Hafiz“ in der US-Botschaft. Vgl. HeIKAL, Return of the Ayatollah, S. $16 \mathrm{f}$.

348 Al-E AhMAD, Gharbzadegi; vgl. HANSON, Westoxication; MiRSEPASSI, Religious Intellectuals; DEYLAMI, Alternative Global Modernity; BOROUJERDI, Triumph of Nativism, S. 65-75; SHAKIBI, Politics of Occidentalism, nennt das Phänomen „Occidentosis“, wohl zu verstehen als „Okzidentitis“ oder „Okzidentoptosis“; die ostentative Ablehnung westlich-modernistischen Denkens war sicherlich auch eine Reaktion gegen die Selbstdarstellung der Herrschenden als willfährige Umsetzungsorgane aufklärerischen Gedankenguts in Iran. Mohammad Rezā hatte in seiner 1960 veröffentlichen, zuvorderst an ein westliches Publikum gerichteten Autobiographie sogar ein Kapitel mit ,Westernization: Our Welcome Ordeal“ tituliert. Siehe ders., Mission for My Country, S. 134; überzogen die Gegenthese in MIRSEPASSI, Iran's Quiet Revolution.

349 Miller Study, S. 19; zu Nasrs Rolle als Dekan der MIT-Kopie Ārjāmehr Unversity of Technology vgl. LESLIE/KARGON, Exporting MIT, S. 123-8; zur sich wandelnden Rolle der Intellektuellen in dieser Phase vgl. NABAVI, Changing Concept.
} 
Konzentration auf die vorislamische persische Zivilisation und das Achämeniden-Reich - die Hinwendung zum schiitischen Islam als Gegenentwurf und alternatives Gesellschaftsideal. Der langjährige Veteran der Nationalen Front, Allāhjār Sāleh, hatte bereits 1944 angesichts zunehmender Einmischung der USA in die inneren Angelegenheiten seines Landes seine zuvor kritiklos pro-amerikanische Haltung revidiert. Im Kontext der nun fast uneingeschränkten Identifikation der USA mit der Schah-Herrschaft aber wandelte sich Sālehs bereits recht distanziertes und kritisches, aber doch auch aufgeschlossenes Amerikabild in Feindseligkeit. Die CIA berichtete Ende 1966, Sāleh sei intern dazu übergegangen, die USA als kolonialistische und repressive Macht zu bezeichnen. ${ }^{350}$ Kaum noch zu finden waren Ende der 1960er Jahre iranische Intellektuelle, die die USA als ein Vorbild ansahen, dem es nachzueifern galt. Ein Vierteljahrhundert amerikanischer Unterstützung für die Herrschaft Mohammad Rezās hatten den traditionellen Wohlwollen gegenüber Amerika abgetragen.

Ein gleichermaßen entscheidendes und einschneidendes Ereignis, das zur Absenkung des Ansehens der Vereinigten Staaten in den Augen großer Teile der iranischen Bevölkerung maßgeblich beitrug, war die sogenannte Status Bill-Affäre im Oktober-November 1964, in deren Mittelpunkt die Frage der rechtlichen Aspekte der ausländischen Militärpräsenz in Form der immer zahlreicher werdenden amerikanischen Militärberater stand. Von vielen Beobachtern als sekundär empfunden, war diese Frage in Realität in einem lange Zeit zu erheblichem Souveränitätsverzicht genötigten Staat wie Iran mit einer hundertjährigen Geschichte - vom Frieden von Turkmantschai von 1828 bis zur formalen Aufhebung durch Rezā Šāh 1927/28 351 - eines von außen durch „Kapitulationen“ und „ungleiche Verträge“ aufgezwungenen Regimes von Extraterritorialität von größter politischer Brisanz. Gerade die Frage von Privilegien für Ausländer wie die Befreiung von der Polizeigewalt und der Gerichtsbarkeit des Gaststaates wurde in den kulturellen Kontexten von Nationalismus und Dekolonisation zu einem kontroversen Gegenstand innenpolitischer Auseinandersetzungen.

Tatsächlich war die amerikanische Beraterpräsenz im Kontext der historischen Erfahrungen Irans alles andere als unumstritten - so blieb auch der rechtliche Status der während des Zweiten Weltkriegs in Iran stationierten US-Soldaten aufgrund der Unvereinbarkeit der Standpunkte bis zu deren Abzug ungeklärt. Die Frage strafrechtlicher (aber auch zivil- und verwaltungsrechtlicher) Immunität der in Iran verbliebenen US-Berater und ihres Familienanhangs blieb auch im Anschluss ohne formale Regelung. Die US-Beratermissionen bei der iranischen Gendarmerie (GENMISH) und die Militärmission (ARMISH) basierten im Kern auf den vertraglichen Vereinbarungen aus dem Zweiten Weltkrieg. Einige Berater waren formal Teil der MAAG (seit 1952) auf Basis des Mutual Defense Assistance Agreement vom Mai 1950, die man zwar mit der bestehenden Militärmission unter dem Kommando eines

${ }_{350}$ CAS $\{C I A\}$ Memo to Embassy Chief Political Section, 6.12.66, Asnād 21, 2, S. $109 f$.
${ }^{351}$ Vgl. ZIRINSKY, Abrogation. 
einzelnen Offiziers als ARMISH/MAAG verschmolzen hatte, die aber aus rechtlicher Sicht dennoch als separat anzusehen war. Für alle Militär- und Gendarmerieberater galt somit, dass keine der zugrunde liegenden Vereinbarungen mit Iran die Frage der strafrechtlichen Verantwortlichkeit regulierte. In der Realität war noch nie ein amerikanischer Militärangehöriger zu einer Haftstrafe verurteilt worden bzw. musste jemand einen Aufenthalt in einem iranischen Gefängnis erdulden. In der Regel schafften die Militärmissionen amerikanische Beschuldigte - mit Duldung der iranischen Behörden - möglichst rasch außer Landes und vereitelten so die Strafverfolgung. ${ }^{352}$ Angesichts des Entgegenkommens der Iraner trotz fehlender rechtlicher Grundsatzvereinbarungen plädierte das State Department gegenüber dem mit der fehlenden Regelung unzufriedenen DOD für ein fortgesetztes Tolerieren der unbefriedigenden Situation. Deputy Under Secretary of State Robert Murphy warnte im Juli 1957 vor dem Erzwingen einer grundsätzlichen Absprache. Die Zurückhaltung der Iraner ,[...] to formalize the de facto immunity enjoyed by American servicemen“" sei verständlich. Eine solche formale Vereinbarung müsse dem Mağles zur Ratifikation vorgelegt werden - in einer Parlamentsdebatte aber würde ohne Frage die Ähnlichkeit einer Immunitätsvereinbarung zu den ehemaligen Kapitulationen aus der Zeit des Imperialismus hervorgehoben und attackiert werden. Die Publizität solcher Vorwürfe könnte im Extremfall sogar zum erzwungenen Abzug der Missionen führen, so Murphy weiter. Das DOD beharrte dennoch auf einer formalen Vereinbarung, mit dem Standardmuster des NATO-Truppenstatuts als Minimalforderung. ${ }^{353}$

Seit 1958 waren die bilateralen Vereinbarungen für Gendarmerie- und Militärmission ausgelaufen. Keiner der beiden Verträge wurde verlängert, vor allem aufgrund der ungeregelten Frage des rechtlichen Status der Missionsmitglieder. ${ }^{354}$ Das State Department verwies vergeblich auf die Tatsache, dass in , [... ] no past case has a United States serviceman been subjected to confinement after a trial and conviction“" unter den bisherigen informellen

\footnotetext{
352 Vgl. MotTer, Persian Corridor, S. 439-46; die Gendarmerie-Mission war wie die Beratermission für die iranischen Streitkräfte seit 1942 in Iran aktiv. Die Vereinbarung für GENMISH war am 27. November 1943 in Kraft getreten (rückwirkend gültig seit dem 2. Oktober 1942) und seitdem unregelmäßig verlängert worden, das letzte Mal im Februar 1956. Das Vereinbarung lief mangels formaler Verlängerung am 20. März 1958 aus. ARMISH war am 6. Oktober 1947 in Kraft getreten und die letzte Verlängerung ebenfalls am 20. März 1958 ausgelaufen. Die Vereinbarung für MAAG hatte keine zeitliche Befristung. Vgl. Note ,Summaries [... $]^{\star}$, o.D., , 3A. Armish/MAAG Mission, 1962‘, NEA, GTI: RIAD, 1958-63, Box 8; eine Bestandsaufnahme des Ist-Zustands im Department of Defense kam zu dem Schluss: ,we have no legal protection for our forces in Iran, and little reliable protection on other grounds."; Vgl. Rubin Memo, 12.5.61, FRUS 61-63, XVII-XVIII-XX-XXI Suppl., Dok. 60.

${ }^{353}$ Vgl. Murphy Letter, 9.11.57, 788.5/7-3157; vgl. Sprague Letter, 31.7.57, , Iran Security Matters - General 1957'; Lot 60 D 533, Subject Files Relating to Iran, 1951-1958, OiCIA/GTI, Box 10; im Anschluss an den sogenannten Girard Case, der Anklage eines GIs vor einem japanischen Gericht, verstärkte sich der Widerstand innerhalb der US-Öffentlichkeit und insbesondere im US-Kongress gegen die Status-of-Forces Agreements im Allgemeinen, vermutlich der eigentliche Hintergrund der DOD-Forderungen bez. Irans. Vgl. PFAU, Legal Status, S. 144f.

${ }_{354}$ Wie zu erwarten, drängte das Defense Department auch 1958 wieder auf eine formale Vereinbarung, allerdings nun eine Immunität nach diplomatischen Muster gegenüber einem formalen Status-of-Forces Agreement präferierend und sich auf einen NSC-Beschluss vom März 1958 beziehend, der ungeregelte Rechtsverhältnisse wie im iranischen Fall untersagte. Vgl. McElroy Letter, 28.5.58; Nussbaum Memo, 1.10.58; ,[Iran] Status of Forces [1958] 3-A‘, Lot 60 D 533, Subject Files Relating to Iran, 1951-1958, OiCIA/GTI, Box 10; Sprague Letter to Dillon, 4.9.58, 788.5/9-458, CF 55-59.
} 
Absprachen mit den Iranern. ${ }^{355}$ Die amerikanischen Berater, die Mitglieder dieser Missionen waren, hielten sich seitdem mit einem ,abnormen“ Status in Iran auf, also ohne vertragliche Basis, wenngleich Artikel II des bilateralen Abkommens von 1959 indirekt auf die Beratungstätigkeit Bezug nahm und beide Seiten von einer automatischen Verlängerung der bisherigen Vereinbarungen auf jährlicher Basis auszugehen schienen, doch monierten die Juristen im State Department und Pentagon diese rechtliche Basis für deren Präsenz als ungenügend. Eine der wesentlichen Streitfragen zwischen beiden Regierungen, welche die Verhandlungen Ende der 1950er Jahre blockierten, war die bereits oben behandelte iranische Forderung - in 1948 und erneut in 1957 - nach Revision des Artikels 24 der ARMISHVereinbarung, der das amerikanische Beratungsmonopol festschrieb. Eine solche obligatorische Festlegung auf die Wahrnehmung von Beraterdiensten ausschließlich vonseiten der USA war iranischen Nationalisten von jeher ein Dorn im Auge gewesen. Mit einem Notenaustausch im Jahr 1960 hatte man einen Kompromiss gefunden, der zumindest diese Frage etwas entschärfte. Im Falle der iranischen Absicht, die Dienste anderer Staaten in Anspruch zu nehmen, genügte nunmehr eine vorhergehende Notifikation der amerikanischen Regierung. ${ }^{356}$ Umstritten blieben jedoch zwei Sachverhalte. Seit dem sogenannten Mansfield Amendment von 1954 des Mutual Security Act von 1951 war die direkte Kompensation amerikanischer Berater durch die Gastregierung untersagt. Die US-Berater in Iran wurden jedoch bislang direkt seitens der iranischen Regierung entlohnt - allerdings gab es eine Reihe von Ideen, dieses Problem zu umgehen. Das Hauptproblem aber war weiterhin die Frage strafrechtlicher Immunität der Berater und ihrer Angehörigen. Schah Mohammad Rezā Pahlavi und der Stabschef der Armee hatten im Oktober und November 1960 auf mündlicher Basis eine vertrauliche, provisorische Abmachung mit den Amerikanern getroffen, wonach rechtlich zwischen Gesetzesübertretungen begangen während Ausübung des offiziellen Dienstes und „off duty offenses“" unterschieden werden sollte. ${ }^{357}$

Die Juristen der Administration bewerteten den Rechtsstatus der US-Berater selbst bei Einhaltung dieser Zusagen als unvorteilhafter im Vergleich zum NATO-Truppenstatut von 1951 und dem Zusatzabkommen von 1959. Gemäß eines NSC-Beschlusses vom November 1960 hätten die US-Berater daher abgezogen bzw. die Administration hätte Iran ausdrücklich als Ausnahmefall (infolge eines ,overriding national interest“) definieren müssen. Im Laufe von 1961 drängte das Department of Defense daher auf eine Wiederaufnahme der Verhandlungen mit den Iranern, während das State Department vorwiegend aus politischem

\footnotetext{
${ }^{355}$ Becker Letter, 5.1.59, 788.58/12 -858; das DOD beharrte Ende 1958 gegen den Rat des State Department auf einer sofortigen Initiative mit dem Ziel einer vertraglichen Zusicherung von „exclusive criminal jurisdiction“ über das eigene Personal im Anschluss an eine Verlängerung von ARMISH und GENMISH. Daran scheiterte die angestrebte unbegrenzte Verlängerung. Vgl. Knight Memo, 10.12.58, 788.58/12-1058; State Instruction \#A-120, 19.1.59, 788.58/1-1959; Irwin II, Letter to Dillon, 21.4.59, 788.58/4-2159, ebd.; offenbar betrachteten beide Regierungen trotz fehlender formeller Absprachen die alten Vereinbarungen als sich quasi-automatisch selbst jeweils um ein Jahr verlängernd. Siehe Rubin Memo, 12.5.61, Anm. 352. ${ }^{356}$ Vgl. Rubin Memo, 1.2.61, ,3-A. Status of Forces 1961', NEA, GTI: RIAD, 1958-63, Box 2.

${ }^{357}$ Vgl. Mouser Memo, 18.5.59; Peterson Memo, 14.5.59, ,Iran, Status of Forces 1959`, ebd., Box 1; Sana Tehran \#091100Z JW 49, 10.12.60, CF 60-63, 788.00(W)/12-960.
} 
Kalkül für die Beibehaltung der informellen Abmachungen plädierte. ${ }^{358}$ Dagegen protestierte die militärische Seite: Iran sei ,[...] the only country in the world in which the local authorities may try U.S. military forces.“359 Im März 1962, wieder im Kontext der anvisierten Verlängerung amerikanischen Militärmissionen in Iran und ausgelöst durch erstmalige, kurzzeitige Inhaftierungen amerikanischer Militärangehöriger in Iran, drängte die USAdministration zum ersten Mal mit Nachdruck auf den Abschluss einer bilateralen Aufenthaltsvereinbarung - einem Status of Forces Agreement (SOFA) - und auf die Ausdehnung der Immunitätsvorschriften aus dem gerade erst ausgehandelten Wiener Übereinkommen über diplomatische Beziehungen von 1961 auf die Mitglieder der USMissionen. Zwar war damit kein vollständiger Diplomatenstatus, sondern der im Übereinkommen definierte begrenztere Status für Verwaltungskräfte und technisches Personal gemeint. In der Umsetzung aber hätte dies strafrechtliche Immunität für alle US-Berater und deren Familienangehörige impliziert (,,acceptance of this proposal would [...] give us full jurisdiction over U.S. military personnel involved in criminal cases"), den Souveränitätsverzicht im Vergleich zu den NATO Truppenstatuten also noch weit übertreffend. Das iranische Außenministerium hatte erwidert, ein Zugeständnis von solch weitreichender Immunität von iranischer Strafgerichtsbarkeit sei ,politisch unmöglich“ - der damalige proamerikanische Premier 'Ali Amini hatte sich auf Anraten des Justizministeriums dem Ansinnen verweigert. ${ }^{360}$ Auch auf amerikanischer Seite war man sich der möglichen Tragweite der ausgeweiteten Immunität durchaus bewusst. In einem Brief an William Bundy im Pentagon warnte Assistant Secretary Talbot explizit:

If and when the Iranians accept this proposal, criminal jurisdiction will in effect be given to the American authorities. [...] We know, however, Iran's long history of suspicion about all forms of foreign involvement, especially any involvement which seems to them to be an infringement on Iranian sovereignty. Once it is agreed upon and becomes public knowledge, this jurisdictional agreement will be considered by some articulate Iranians as just such an infringement. ${ }^{361}$

\footnotetext{
${ }^{358}$ Vgl. Jones Memo, 7.5.59, ,Iran, Armish (MAAG) Mission 1959‘, NEA, GTI: RIAD, 1958-63, Box 1; Rubin Memos, 15.2.61, 23.2.61, ,3-A. Status of Forces 1961', NEA, GTI: RIAD, 1958-63, Box 2; Assistant Secretary Talbot warnte, dass eine erzwungene Formalisierung der Regelungen sehr wohl in einer Minderung des rechtlichen Schutzes für das amerikanische Personal resultieren könne. Siehe Talbot Letter, 10.7.61, ebd.; neben den etwa 500 Militärberatern gab es auch noch zehn weitere, von den Beratermissionen formal unabhängige US-Einheiten im Land, mit etwas über 300 Mann Kaderstärke. Vgl. Lemnitzer Memo, 6.3.62, , Iran 1962 111---333‘; U.A. Johnson Letter, 20.3.62, ,Iran 1962 334---680.1`, OASD/ISA Files: General and Country, 1962, Box 89, RG 330; JCS, Decision on JCS 1714/140, o.D. [März 1962], ,CCS 91813410 Iran (29. Nov 1961)‘, JCS Central Decimal File 1961, Box 184, RG 218, NACP.

${ }^{359}$ MemCon, 28.9.61, 788.5 MSP//9-2861, CF 60-63; Barbis Memo, 29.9.61, ,3/A. Status of Forces 1961 ', NEA, GTI: RIAD, 1958-63, Box 2; mit Ausnahme der Türkei und Iran besäßen amerikanische MAAGs in 58 von 60 Staaten diplomatischen Status und damit Immunität.

${ }_{360}$ Vgl. State \#626, 17.3.62, FRUS 61-63, XVII-XVIII-XX-XXI Suppl., Dok. 85; der Widerstand des State Department war nun gebrochen: „If a growing sentiment in the Pentagon to slash our MAP ever converges with Congressional anger over jurisdictional questions, we could be put in an extremely dangerous position. "; Miner Letter, 22.1.62, ,3-A. Status of Forces, 1962‘, NEA, GTI: RIAD, 1958-63, Box 6; die iranische Gerichtsbarkeit würde sich somit nur noch auf zivilrechtliche Fälle im Zusammenhang mit Vorfällen außerhalb der Dienstzeiten beziehen, was auch für das DOD akzeptabel war. Die Botschaft warnte weiterhin vor einem Drängen auf ein SOFA in Iran. Selbst für den unwahrscheinlichen Fall, dass die iranische Seite ein solches akzeptieren würde, bestünde im Anschluss die Möglichkeit, US-Militärpersonal , ,...] would be serving prison sentences under conditions abhorrent by U.S. standards.“; Oulashin Letter, 26.8.61, ,Letters from Tehran 1961 ', ebd., Box 2.

${ }^{361}$ Talbot Letter, 9.3.62, ,Iran $1962111---333^{\circ}$, OASD/ISA Files: General and Country, 1962, Box 89, RG 330; vgl. Rubin Memo, 30.3.62, ,3-A. Status of Forces, 1962‘, NEA, GTI: RIAD, 1958-63, Box 6; Bill Millers späterer Vorwurf, wonach ,for most Americans, the insensitivity to capitulation [...] in the late 19th century - and the
} 
Trotz der Absage Aminis und der grundsätzlichen Bedenken im State Department drängte das DOD weiterhin auf ein formales SOFA und darüber hinaus nun auch auf ein Junktim zwischen einem solchen und der Fortsetzung der MAP-Lieferungen. ${ }^{362}$ Gleichzeitig verstärkte sich der Druck vonseiten des US-Kongresses. ${ }^{363}$ Die US-Administration verfolgte weiterhin die Idee der Verknüpfung der Immunitätsfrage mit dem Wiener Übereinkommen. Der Druck zahlte sich letztlich aus. Im März 1963 informierte das iranische Außenministerium die Botschaft in einer diplomatischen Note, man akzeptiere nun diplomatischen Status für die hochrangigen Mitglieder der Militärmissionen. Im November folgte eine weitere Note, die dem amerikanischen Wunsch nach Ausdehnung der im Wiener Übereinkommen vorgesehenen Privilegien und Immunitäten auf sämtliche Mitglieder der Missionen und ihre Angehörigen entsprach. $^{364}$ Eine entsprechende Konkretisierung sollte dem neu gewählten Mağles gemeinsam mit dem Wiener Übereinkommen zur Ratifikation vorgelegt werden.

Die US-Botschaft in Teheran, selbst unter Druck seitens des DOD, drängte nun auf eine baldige Ratifizierung durch das Parlament. Erste Warnsignale während der Debatte des Übereinkommens und des begleitenden Notenaustausches mit den USA im Senat im Sommer 1964 wurden ignoriert. Man erwartete einen reibungslosen Abschluss des Ratifikationsprozesses, zumal die Mansur-Regierung die Unterstützung von drei Vierteln der Abgeordneten besaß und auch der Schah selbst im September 1964 keine Schwierigkeiten für die Verabschiedung im Mağles erwartete. ${ }^{365}$ Vor dem Beginn der Debatte im Plenum im Oktober häuften sich alarmierte Berichte der Botschaft über um sich greifenden Widerstand vieler Abgeordneter gegen den Umsetzungsbeschluss mit Bezug auf die USMilitärangehörigen, in der Regel als Status Bill bezeichnet, und der Vorwurf von „Kapitulationen“ nach dem historischen Vorbild von Turkmantschai machte die Runde. ${ }^{366}$ Premierminister Hasan 'Ali Mansur unterschätzte den parlamentarischen Widerstand und vertraute auf die Dominanz seiner Partei des Neuen Iran (Hezb-e Irān-e nowin) in der Kammer. Umso gewaltiger war der Schock im Anschluss an die geheime Abstimmung am 13. Oktober. Zwar gab es eine Mehrheit für die Ratifizierung, doch fiel diese mit 74:61 Stimmen unerwartet knapp aus - zudem war die Debatte im Plenum von anti-amerikanischen Ausfällen geprägt gewesen. Zwar berichtete die Botschaft erleichtert, nun sei die so lange die Beziehungen

whole idea of legal sovereignty - was completely missed“, ist somit unberechtigt; William G. Miller OHI, 7.3.85, IOHP, Transcript 1, S. 30 .

${ }_{362}$ Die Iraner warnten mit Nachdruck vor einer Reaktion eines zukünftigen Mağles auf ein SOFA. Vgl. Schwartz Letter, 26.7.62; Miklos Letter, 24.8.62; ,3-A. Status of Forces, 1962‘, NEA, GTI: RIAD, 1958-63, Box 6.

${ }^{363}$ Vgl. Dutton Letter to Ervin, Jr., 22.5.62, ebd,; vgl. PfAU, Legal Status, S. $146 \mathrm{ff}$.

${ }^{364}$ Text der diplomatischen Noten N॰8800 vom 11.3.63 und No8296 vom 17.11.63 in Tehran \#545, 14.11.64, DEF $15-3$.

${ }^{365}$ Vgl. Tehran \#A-53, 31.7.64, DEF 15-3 IRAN-US, CF 64-66; Memo for the Record, 24.9.64; Gildart Memo,

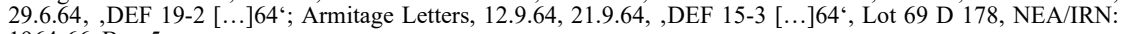
1964-66, Box 5.

${ }^{366}$ Gerade die zunehmende Repression und verschärfte Zensur im Gegensatz zur liberaleren Amini-Ära wirkte sich nun negativ aus. Geheimhaltung der Vereinbarungen und das Verbot jeder öffentlichen Debatte über das Status Bill vor dem eigentlichen Einbringen der Gesetzgebung befeuerten Argwohn und Verschwörungstheorien. Vgl. Tehran \#398, 12.10.64, DEF 15-3 IRAN-US, CF 64-66. 
belastende Frage der diplomatischen Immunität endlich geklärt, doch habe „,not only Mansur but to some extent also the Shah's regime [...] paid an unexpectedly high price in getting this done. ${ }^{\text {367 }}$

Die nächsten Wochen würden zeigen, ob die Umstände der Ratifikation des Status Bill ,longerlasting effects on our relations with Iran" hätten, so die Botschaft - retrospektiv betrachtet handele es sich fraglos um ein ,political event of intrinsic importance“. Die Affäre habe zudem ,[...] the limitations which exist even for a dictatorship in its handling of a volatile and excitable public opinion“ demonstriert. ${ }^{368}$ Es war die Existenz dieser meinungsstarken Gegenöffentlichkeit, welche die US-Offiziellen überraschte und die bisherigen Einschätzungen einer apathischen und willfährigen iranischen Bevölkerung zumindest temporär infrage stellte. Offenbar diskutierte man - im Anschluss an die frühen Debatten im Senat - in den gebildeten Schichten in Teheran Gerüchte über einen geheim gehaltenen, umfangreichen Ausverkauf iranischer Souveränität. Im Anschluss an die Mağles-Abstimmung verbreitete sich dann die Kunde über erzwungene „Kapitulationen“ in Windeseile im ganzen Land. Amerikanische Quellen in entlegenen Gebieten berichteten von aufgeregten Debatten über den vorgeblichen Verrat an der Nation. Die Mansur-Regierung versuchte erfolglos, den gärenden Widerstand mittels einer vollständigen Nachrichtensperre einzudämmen. Nach zwei Wochen gab der Premier diese Taktik auf und versuchte eine Kehrtwende, indem er nun öffentlich die eigene Politik bezüglich der Immunitätsfrage erläuterte. Mansurs Erklärungsversuche trafen auf Misstrauen weiter Teile der Bevölkerung und die US-Botschaft berichtete frustriert von den Schwierigkeiten, selbst pro-amerikanische und gebildete Iraner von der Vorstellung neuerlicher „Kapitulationen“ abzubringen. ${ }^{369}$

367 Tehran \#404, 14.10.64, FRUS 64-68, XXII, S. 104; offenbar waren 50 der insgesamt 138 Abgeordnete der Hezb-e Irān-e nowin nicht anwesend, vermutlich um sich einer Festlegung zu entziehen. Dennoch mussten wenigstens zwölf gegen die eigene Regierung gestimmt haben. Die Partei selbst errechnete einige Tage später die Zahl von 20 Abweichlern. Drei Verkehrsunfälle mit amerikanischer Beteiligung kurz vor den Mağles-Beratungen trugen zur Emotionalität der Debatten bei. Vgl. Tehran \#448, 27.10.64; Tehran \#A-195, 30.10.64, DEF 15-3 IRAN-US, CF 64-66.

${ }^{668}$ Zitat aus Telegram \#404; die anderen Zitate aus Airgram \#A-195, beide vorherig. Anm.; „The Government was taught the lesson that even a rubber-stamp parliament sometimes behaves almost like a real parliament [...]."; Mansur habe aus übertriebenem Optimismus heraus auf eine direkte Weisung des Schahs an die Abgeordneten verzichtet. Outgoing Tehran \#A-303, 10.12.64, ,Iran - Airgram/Reports, 1963-1967`, Part 2: Box 1a, Herz Papers, GUL; eine andere Quelle suggeriert eine andere Lesart. Mansur habe angesichts der Unpopularität des Status Bill bewusst und in Absprache mit dem Monarchen auf eine Instruktion des Hofes verzichtet und damit die Verantwortung in Gänze selbst übernommen. Vgl. Lorenz Memo, 20.4.66, ,DEF 15-3 Status of Forces IRAN 1966', Lot 70 D 330, NEA/IRN: 1964-66, Box 17.

369 „Public opinion in Iran is difficult to gauge, but also most unwise to ignore.”; \#A-303, vorherig. Anm.; Mansurs Erklärung vor dem Senat enthielt eine ganze Reihe von falschen Behauptungen mit Bezug auf die Reichweite des Status Bill, was wiederum die US-Botschaft im Anschluss zwang, auf rechtlich verbindliche Klärungen bezüglich des diplomatischen Notenwechsels zu drängen. Vgl. Tehran \#499, 3.11.64, FRUS 64-68, XXII, S. 108ff.; Mansur korrigierte einige, wenngleich nicht alle Fehldarstellungen in einer weiteren Rede. Ein erneuter Notenaustausch im Dezember bestätigte sodann die amerikanische Lesart der Reichweite im Gegenzug zu einer freiwilligen Konzession Washingtons, in besonderen Fällen (,,heinous cases"), also bei gravierenden Rechtsbrüchen wie Mord oder Vergewaltigung, einen unilateralen Verzicht auf Immunität in Erwägung zu ziehen. Aus völkerrechtlicher Sicht war das Status Bill bis zum Abschluss der amerikanischen Ratifikation des Wiener Übereinkommens im Jahr 1972 eigentlich nicht in Kraft, doch beharrte das State Department wider besseren Wissens auf sofortige Gültigkeit. Zu den rechtlichen Nachklärungen vgl. Tehran \#495/\#A-306, 2.11./12.12.64; State \#1031, 14.5.65, DEF 15-3 USIRAN, CF 64-66; Herz Letter, 5.11.64; Memo, , Background Summary‘, 6.11.64, ,DEF 15-3 Status [... 164', Lot 69 D 178, Box 5; Mulligan Memo, 11.6.65, ,DEF 15-3 [...]65', Lot 69 D 490, NEA/IRN: 1964-66, Box 12; Tehran \#643, 9.12.64, FRUS 64-68, XXII, S. 114f.; die Wertung der Nachverhandlungen als Beweis für den ,lack of real 
Schließlich verlieh Ājatollāh Khomeini der Unzufriedenheit in der Bevölkerung über das Status Bill eine Stimme, das von der Mansur-Regierung überlassene politische Vakuum kraftvoll füllend. Seit April 1964 wieder in Freiheit, wenngleich unter der Auflage der Einstellung jeglicher politischer Aktivitäten, ergriff er die Gelegenheit, das Status Bill und den Schah selbst massiv anzugreifen. In einer Rede am 27. Oktober bezichtigte er die Führung des nationalen Verrats infolge der Gewährung von „Kapitulationen“ an eine auswärtige Macht und der entwürdigenden Aufgabe staatlicher Souveränität:

The have reduced the Iranian people to a level lower than that of an American dog. If someone runs over a dog belonging to an American, he will be prosecuted. Even if the Shah himself were to run over a dog belonging to an American, he would be prosecuted. But if an American cook runs over the Shah, the head of state, no one will have the right to interfere with him. ${ }^{370}$

Das Status Bill sei gesetzeswidrig, schändlich und, darüber hinaus, sei es unvereinbar mit dem Koran und dem islamischen Glauben - der Geistliche rief die Armee unverhohlen zum Sturz der Regierung auf. Wenig überraschend wurde Khomeini umgehend festgesetzt - wider Erwarten fiel die Bestrafung aber vergleichsweise moderat aus, offenbar auf Betreiben des SAWAK-Direktors Pākrawān, der sich später rühmte das Leben des Ājatollāhs gerettet zu haben. Khomeini wurde in die Türkei zwangsexiliert, von wo er nach etwas weniger als einem Jahr weiter in den Irak reiste. Die staatlichen Propagandainstitutionen diffamierten ihn gleichzeitig als Verräter, der versucht habe, so der absurde Vorwurf, das iranische Öl der VAR Nassers zu übereignen. ${ }^{371}$

Trotz Exilierung verbreitete sich der Text von Khomeinis Rede „like wildfire“ in Teheran und den Provinzen, berichtete die Botschaft. Audiokassetten mit seiner Rede würden sogar offen auf dem Basar der Hauptstadt verkauft. Das Vorgehen der Regierung habe Khomeini in den Augen weiter Teile der Bevölkerung die „Aura eines Märtyrers“ verschafft und er sei nun

change“ bei OFFILER, Modernization of Iran, S. 85, ist eine den Quellen widersprechende Fehlinterpretation. Eine iranische Sichtweise auf den Verhandlungsprozess bei MIRFENDERESKI, Privileged American.

${ }^{370}$ Rede Khomeinis in: Algar, Writings and Declarations, S. 181-8, hier 182; ein ähnliches Szenario einer Ermordung eines Marğa’ durch einen amerikanischen „Koch“ oder „Diener“ ausgeführt in ebd., S. 181f.; vgl. MoIN, Khomeini, S. 121-8; Khomeini hatte in der Sache recht. Tatsächlich hatte Iran mit dem Status Bill größere Souveränitätseinbußen akzeptiert als andere Staaten wie z.B. die Türkei und Pakistan. Bezeichnend für den amerikanischen Mangel an Selbstreflektion war die unwahre interne Behauptung, diese Benachteiligung sei eine Folge des iranischen Insistierens auf eine Verbindung mit dem Wiener Übereinkommen und somit selbstverschuldet gewesen. Siehe Tehran \#495, vorherig. Anm.; nur einen Monat zuvor hatte die Botschaft eine Abnahme der „Virulenz“ von Khomeinis Opposition angesichts des ,judicious mix of bribery, conciliatory tactics and the ever-present threat of the regime's mailed fist" berichtet. Tehran \#A-139, 22.9.64, FRUS 64-66, XXII, S. 101; vgl. BILL, Eagle and Lion, S. 158f.; international vergleichbar war eigentlich nur der Minderstatus der Bundesrepublik Deutschland, in der das im übrigen NATO-Raum geltende Truppenstatut durch ein Zusatzabkommen von 1959 (in Kraft 1963), basierend auf besatzungsrechtlichen Sondervollmachten, im Kern unterlaufen wurde. Im Gegensatz zu den anderen NATO-Mitgliedern verzichtete Bonn damit generell auf sein Vorrecht bei der Ausübung der konkurrierenden Gerichtsbarkeit. Wenig bekannt ist, dass im Grundsatz dieser Minderstatus auch nach der deutschen Wiedervereinigung weiterbesteht.

${ }^{371}$ Vgl. Tehran \#508/\#521, 4./5.11.64, FRUS 64-68, XXII, S. 110f., 113f.; zu Pākrawāns angeblicher Intervention vgl. PAKRAvan, Memoirs, S. 38-41; der anti-britischen Verschwörungstheorien zuneigende Schah verdächtigte offenbar den Einfluss Londons hinter der knappen Abstimmung über das Status Bill im Parlament. TELNO 812 to FO, 20.10. 1964, PREM 13/2169, TNA:PRO. 
endgültig zu einem ernstzunehmenden Prätendenten für die Marğa ’ijja, der obersten Autorität für die schiitischen Gläubigen, aufgestiegen. Eine Analyse der Botschaft konstatierte allerdings ominös für den Fall, dass Khomeini eines Tages nach Iran zurückkehre, „[...] he would no doubt find a more enthusiastic following than he had before his exile. ${ }^{\text {"372 }}$ Bemerkenswert sei ohnehin, wie sehr sich die Wahrnehmung der religiösen Opposition infolge der Affäre verändert habe. Vormals als reaktionär und rückwärtsgewandt in ihrem Widerstand zu Frauenwahlrecht und Landreform verspottet, werde die Geistlichkeit nun sogar von säkularen Kräften als glaubwürdiger Vertreter und Verteidiger patriotischer Positionen wahrgenommen. ${ }^{373}$

Zusätzlich aufgeheizt wurde die Atmosphäre durch die weite Verbreitung findende Legende, die Zustimmung des Mağles zu amerikanischen „Kapitulationen“ sei eine direkte Gegenleistung für Washingtons Zustimmung zur weiteren Aufrüstung Irans im Rahmen des \$200 Millionen-Militärkredits, basierend auf dem bereits oben analysierten MOU vom Juli 1964, gewesen. In der Tat hatte der Mağles nur zwei Wochen nach dem Status Bill - dieses Mal allerdings einstimmig - einem Gesetz zugestimmt, das die Aufnahme des Militärkredits autorisierte. Tatsächlich war die zeitliche Nähe rein zufällig, zumal alle im Zusammenhang mit dem Militärkredit stehenden Entscheidungen bereits Monate zuvor gefallen waren. Dennoch hielt sich die Legende eines quid pro quo in der iranischen Öffentlichkeit und, angesichts der eindeutigen Quellenlage überraschend, auch in der Forschungsliteratur bis zum heutigen Tag. ${ }^{374}$ Innenpolitisch wirkte die Status Bill-Affäre in Iran lange nach. Die Ermordung Mansurs im Januar 1965 durch einen religiösen Fanatiker war eine kurzfristige Folge. Aus längerfristiger Perspektive diskreditierte die Affäre das Schah-Regime, aber auch dessen amerikanische Schutzmacht. Die Botschaft konstatierte eine Beschädigung des eigenen Image in Iran: „While it is certainly not true that the image of the United States has taken on the coloration of the British image during the days of the great oil controversy, we must accept the judgment of all our political contacts that our position in Iran has been significantly eroded, at least for the time being, by the Status Bill fiasco.“375 In der inneriranischen Wahrnehmung

372 Airgram \#A-303, S. 558, Anm. 368; es gab zwischenzeitlich sogar Befürchtungen, Khomeini könne beabsichtigen, in die USA überzusiedeln Vgl. Bracken Memo, 10.11.64, ,Office Memoranda', Lot 69 D 30 , NEA/IRN: 1964-66, Box 1.

${ }^{373}$ Vgl. Airgram \#A-303, S. 558, Anm. 368.

${ }^{374}$ Vgl. FRUS 64-68, XXII, S. 108 n3; die CIA berichtete von einem ,unfortunate bit of timing“; Current Intel Memo OCI No. 1109/65, 23.4.65, ebd., S. 141f.; so z.B. explizit bei JOHNS, Changing Patterns, S.70; auch PFAU, Legal Status, S. 150; bizarr zu nennen ist die Wertung bei COHEN, Balancing Interests, S. 287, demzufolge ein ,'brouhaha' late in 1964 over a routine status-of-forces agreement, granting American military personnel in Iran immunity from local persecution, was perceived correctly by analysts in Washington as an effort by opposition fundamentalist forces, led by the Ayatollah Ruhollah Khomeini, to embarrass the government."; apologetisch die Behauptung von Rubin und Pollack, das Status Bill sei identisch zu ähnlichen Vereinbarungen in anderen Staaten gewesen bzw. sogar schlicht unwahr die Behauptung, Mosaddeg habe ein identisches SOFA akzeptiert. Vgl. RUBIN, Good Intentions, S. 111; PollaCK, Persian Puzzle, S. 93; COLLIER, Nature of Influence, S. 233f., behauptet gegen die Quellenlage, die USA hätten die negativen Effekte der Affäre nicht registriert. Wenngleich ein direktes Junktim zwischen Status Bill und dem Gesetz über die Kreditaufnahme abwegig ist, sind die mittelbaren Bezüge aber nicht von der Hand zu weisen. Mohammad Rezā zentrales Ziel der Aufrüstung machte die Präsenz und die baldige Ausweitung der Missionen unumgänglich und daher lag es in seinem Interesse, innenpolitische Kritik in den USA über eine Immunitätsgewährung abzuschwächen.

375 Airgram \#A-303, S. 558, Anm. 368; Meyer bezeichnete die Verabschiedung des Gesetzes später als „Pyrrhussieg“; Tehran \#229, 28.8.65, FRUS 64-68, XXII, S. 167; zu diesem Zeitpunkt glaubte man sich allerdings in der Lage, die „Missverständnisse“" klären zu können: ,we are busy working on various opinion leaders to lessen 
verdichtete sich nun auch die Gleichsetzung der USA mit dem repressiven Regime. Das langjährige Mitglied des pro-amerikanischen Netzwerks in Iran Abu 1-Hasan Ebtehāğ, warnte denn auch 1965 in einem Gespräch mit Walt Rostow, er sehe, ,great danger for the United States in its pro-Shah stance“ und er fügte hinzu, dass , ,...] we will pay a high price later on for our close identification with him. “376 In der Tat blieb die Status Bill-Affäre von diesem Zeitpunkt an ein weithin akzeptierter Bezugspunkt für die Gesamtheit der Opposition gegen die SchahHerrschaft. Ihr Stellenwert bestätigte sich nach der Revolution mit einer direkten Bezugnahme auf die „Kapitulationen“ in der Präambel der Verfassung der Islamischen Republik. ${ }^{377}$ Der Einschätzung von James Bill, demzufolge die Ereignisse von 1964 ein ,crucial signpost along the road leading to the ultimate rupture of Iranian-American relations after the Iranian revolution" gewesen seien, ist somit fraglos zuzustimmen. ${ }^{378}$

the black eye we got from the episode."; Herz Letter, 16.12.64, ,DEF 15-3 [...]64", Lot 69 D 178, NEA/IRN: 1964-66, Box 5; die Sowjets versuchten im Anschluss, weiteres Misstrauen gegen die Beratermissionen zu schüren. So fand Ende 1965 ein offenbar gestohlenes U.S. Army-Dokument aus dem Jahr 1960 weite Verbreitung unter iranischen Offiziellen. In dem Dokument, das man intern im Übrigen als authentisch bestätigte, wurden Leiter von Militärmissionen aufgefordert, mit den Attachés der Botschaft eng zu kooperieren, und geheimdienstlich verwertbares Material und Informationen weiterzugeben. Zweifelsohne eine gängige Praxis für jede Art von Militärmission umreißend, hatte das Dokument dennoch in einer von starkem Misstrauen gegen die Umtriebe ,imperialistischer Mächte“ geprägten politischen Kultur wie der iranischen sicherlich einen nicht zu unterschätzenden Effekt. Vgl. Rabida Memo, 7.12.65, ,DEF-Defense Affairs IRAN“, Lot 67 D 195, Records of the G/PM, Subject Files, 1962-1966, Box 22; Tehran \#865, 14.12.65; \#State \#714, 23.12.65, POL IRAN-US, CF 64-66; eine ähnliche Kampagne wurde gegen das Peace Corps unternommen. Ein gefälschtes Dokument, vorgeblich vom Leiter des Korps in Iran, warnte die Freiwilligen vor den wilden Bräuchen der fanatischen Muslime und den weitverbreiteten Geschlechtskrankheiten. Es wurde an eine Vielzahl iranischer Offizieller versandt. Vgl. Tehran \#A-300, 9.12.64, AID (US) 14 IRAN, CF 64-66; vgl. auch KUZICHKIN, Inside the KGB, S. $194 \mathrm{f}$.

${ }^{376}$ Crawford Memo, 30.9.65, ,POL 6-Abol Hassan Ebtehaj 1965‘, Lot 69 D 489, NEA/IRN: 1964-66, Box 11.

377 Siehe TELlENBACH, Verfassung, S. 48, 299; die Geschichte des iranischen SOFA verwandelte sich über die Jahre in ein transnationales Erinnerungsgut in der Nahostregion. Die SOFA-Frage sollte nach der amerikanischen Invasion von 2003 die US-irakischen Beziehungen jahrelang belasten und sie war ein bedeutender Faktor beim letztendlichen, wenngleich nur zwischenzeitlichen Abzug der US-Truppen. Dem ehemaligen Deputy Assistant Secretary of State for Iran John Limbert zufolge war während der SOFA-Verhandlungen allen Offiziellen auf der irakischen Seite die historische Präzedenz von 1964 gegenwärtig. Als Limbert daraufhin den US-Botschafter Ryan Crocker nach seiner Meinung fragte, wie viele Offizielle auf US-Seite die Episode wohl kennen würden, antwortete dieser: ,Two. You and me.“; Email von J. Limbert, 14.2.2018.

${ }^{378}$ BILL, Eagle and Lion, S. 156.; ähnlich BARRETT, Greater Middle East, S. $244 \mathrm{f}$. 